\title{
Evaluation of different mycobacterial species for drug discovery and characterisation of novel inhibitors of
}

\author{
Mycobacterium tuberculosis
}

\author{
A thesis submitted by \\ Mudassar Altaf \\ In fulfilments of the requirements for the degree of \\ Doctor of Philosophy \\ in Molecular Microbiology

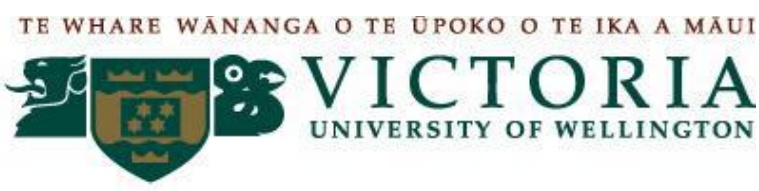 \\ School of Biological Sciences \\ Victoria University of Wellington, New Zealand \\ Te Whare Wānanga o te Ūpoko o te Ika a Māui




\section{Scientific Advisors:}

\section{1- Dr. Ronan O'Toole}

Senior Lecturer, School of Biological Sciences

Victoria University of Wellington

Wellington, New Zealand

\section{2- Prof. John H. Miller}

School of Biological Sciences

Victoria University of Wellington

Wellington, New Zealand

\section{3- Dr. David Bellows}

Senior Lecturer, School of Biological Sciences

Victoria University of Wellington

Wellington, New Zealand 
"It is a sad reflection on society's incompetence that, more than thirty years after the methods for cure and prevention were evolved and before the advent of the HIV epidemic, there were already more patients with active tuberculosis in the world than there had been in the 1950s"

(John Crofton) 


\begin{abstract}
Tuberculosis $(\mathrm{Tb})$ has plagued mankind for many centuries and is still a leading cause of death worldwide. A worrying development is the emergence of drug-resistant $\mathrm{Tb}$ that poses further challenges to the control of the disease. The global $\mathrm{Tb}$ burden and high mortality rate indicate that new drugs are needed for $\mathrm{Tb}$ treatment. While no new anti- $\mathrm{Tb}$ agents have been introduced to the market for about three decades, drugs with novel mechanisms of action can amend the current $\mathrm{Tb}$ treatment regimen and may provide an effective solution to drug resistance.
\end{abstract}

The main objectives of this study were to identify an appropriate in vitro model that could be used for anti-Tb drug high-throughput screening (HTS), and to use this model to identify a novel candidate anti-tubercular drug and its cognate cellular target. A sensitive growth inhibition assay was set up with a GFP-labelled $\mathrm{Tb}$ vaccine strain, $M$. bovis $\mathrm{BCG}$, using standard first and second line anti-tubercular drugs. HTS of the drug libraries was performed with various in vitro models to evaluate their efficacy for use in anti-Tb drug discovery. Approximately $50 \%$ of the $M$. tuberculosis inhibitors were not detected in screening with the surrogate species, $M$. smegmatis; whereas, only $21 \%$ of hits were not detected with $M$. bovis BCG. A comparative genomic study revealed that $97 \%$ of $M$. bovis BCG proteins, compared to $70 \%$ in $M$. smegmatis have conserved orthologues in M. tuberculosis H37Rv. Therefore, $M$. bovis BCG represented a more sensitive model than $M$. smegmatis for detecting anti- $M$. tuberculosis compounds. M. bovis BCG was then used to screen for novel anti-Tb agents by HTS of compound libraries and various plant extracts, followed by validation of new compounds in $M$. tuberculosis H37Ra. A number of novel M. tuberculosis inhibitors were identified, including sappanone A dimethyl ether from plant sources and compounds NSC112200 and NSC402959 from NIH chemical libraries. The inhibitors that were validated 
using M. tuberculosis H37Ra were also validated in the virulent Tb strain, M. tuberculosis H37Rv. In addition, their activity was further investigated using a suite of other clinically important human pathogens.

One of the key anti-mycobacterial hits identified in this study, NSC402959, has previously been detected in screens for compounds that inhibit ribonuclease $\mathrm{H}$ (RNase $\mathrm{H})$, an enzyme that is required for a number of essential cellular processes. NSC402959 inhibited RNase H proteins from HIV as well as from E. coli. Since HIV and Tb are major pandemics, previously-known anti-HIV RNase $\mathrm{H}$ compounds were imported and tested for their antiproliferative activity towards M. tuberculosis H37Ra. HIV RNase H inhibitors, NSC35676, NSC112200, NSC133457 and NSC668394, exhibited good anti-mycobacterial activity in this study. In silico analysis suggested a plausible interaction of these inhibitors with mycobacterial RNase HI. A biochemical assay further confirmed NSC112200 to be specific against RNase HI from $M$. tuberculosis. These interesting inhibitors were not only structurally different from existing anti-Tb drugs but some of them were also non-toxic to mammalian cells and may have a unique mechanism of action. Thus, these compounds showed good potential for development as dual inhibitors of Tb and HIV; therefore, future studies in animal infection models to determine their dual anti-mycobacterial and anti-HIV activities are warranted. 


\section{Acknowledgements}

First and foremost, I would like to thank my supervisors, Dr. Ronan O’Toole and Dr. David Bellows for their continuous support and guidance. I must thank Dr. Ronan for testing my novel compounds against the virulent Tb models in vitro at Johns Hopkins University and for his ideas and important piece of advice throughout my research work.

I gratefully acknowledge the kind help of Prof. John Miller for reviewing my thesis. He has been a tremendous support during my stay at Victoria University. I would like to thank Dr. Paul Teesdale-Spittle for his technical help on in silico analysis of novel inhibitors with mycobacterial RNase HI. I am also thankful to Prof. Paul Atkinson for giving me the opportunity to use state-of-the-art facilities of Chemical Genetics Laboratory. CyBio-96 ${ }^{\circledR}$ robot assistance greatly facilitated the high throughput screening part of this research.

I am also thankful to all my colleagues; Shahista Nisa, for proofreading chapters from my thesis; Chris Miller, for sharing reagents, plasmids and bacterial strains whenever needed and also for sharing his experience of cloning experiments and brilliant scientific ideas; Rekha Murikoli and Nathaniel Dasyam, for their help with setting up cytotoxicity assays; Ian Bassett for dormicidal assays and Richard Campen, for his useful scientific discussions and also for ordering laboratory consumables. Thank you to the rest of KK 817 and KK 815 Microbiology laboratory crew for any help they offered during my bench work. I also thank Laura Green, Jessica Field, Ariane Chan, Bridget Simonson and Dora Leahy for providing HL-60 and J774.A1 cell lines and for their assistance with an MTT assay.

I gratefully acknowledge Victoria University PhD Scholarships for providing an opportunity to conduct doctoral studies in Wellington, without which, it might not have been easy for me to come this far in my academic research. 
I would like to take this opportunity to specially thank the management of the National Institutes of Health (NIH) for providing individual compounds for my hit-validation studies. It would not have been possible to complete this project without these compounds. I also humbly acknowledge kind help of our scientific collaborators at Johns Hopkins University, USA and at The University of Auckland, New Zealand with virulent Tb and ribonuclease $\mathrm{H}$ proteins studies, respectively.

Last but not least; I am very thankful to my beloved family, my parents, siblings and my wife, Aseiah, for their unconditional love, generous help and constant support.

Mudassar Altaf

Mudassar.Altaf@gmail.com 
I dedicate this thesis to my beloved parents 


\section{Table of Contents}

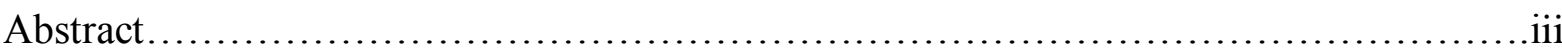

Acknowledgements.............................................................

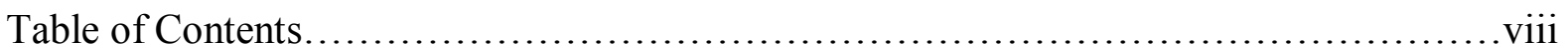

List of Figures.....................................................................

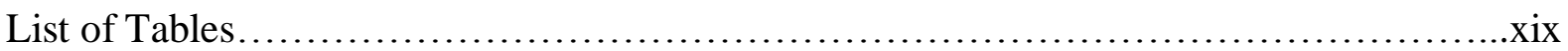

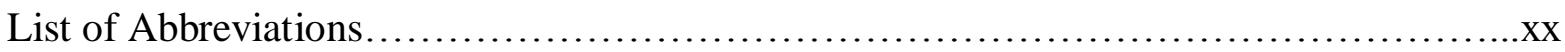

List of Publications...............................................................

Chapter One: General Introduction to Tuberculosis Biology and Drug Discovery........1

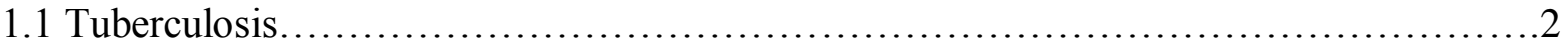

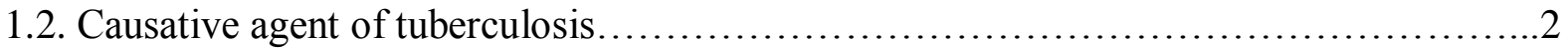

1.2.1. Comparison of latent $\mathrm{Tb}$ infection and active disease $\ldots \ldots \ldots \ldots \ldots \ldots \ldots \ldots \ldots \ldots$

1.3. Mycobacterium tuberculosis complex...........................................5

1.3.1. Mycobacterium tuberculosis...........................................5

1.3.1.1. Morphology and staining properties.................................

1.3.1.2. Cell wall of $M$. tuberculosis.............................................. 8

1.3.1.3. Phylogeography of $M$. tuberculosis................................9

1.3.2. Mycobacterium bovis.............................................. 9

1.3.2.1. Mycobacterium bovis BCG..........................................

1.3.2.1.1. Tuberculosis vaccine ....................................... 10

1.3.3. Mycobacterium africanum ...............................................11

1.3.4. Mycobacterium leprae ..................................................11

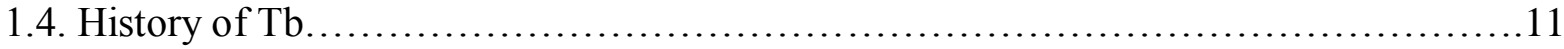

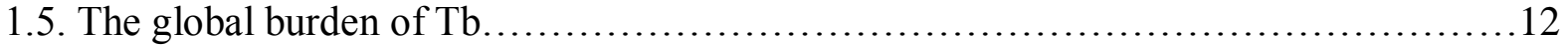

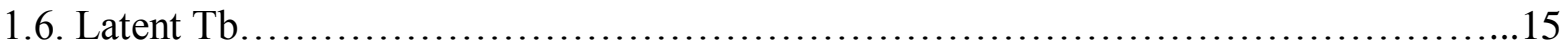

1.6.1. M. tuberculosis dormancy and persistence................................... 15 
1.7. $\mathrm{Tb}$ in New Zealand................................................................

1.8. $\mathrm{Tb}$ treatment, therapeutics and control measures................................. 19

1.8.1. Case diagnosis..................................................... 19

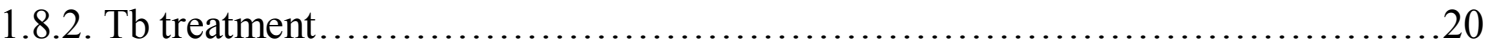

1.8.2.1. Standard anti-Tb drugs..........................................20

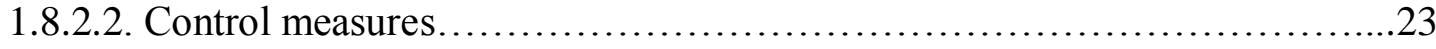

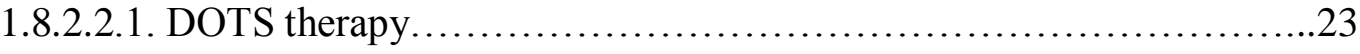

1.8.3. Drug resistance....................................................... 24

1.8.3.1. Resistance due to genetic mutations................................25

1.8.3.2. Phenotypic drug resistance........................................26

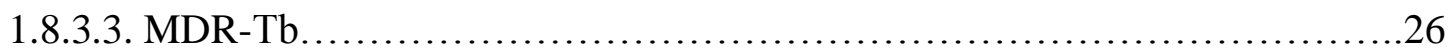

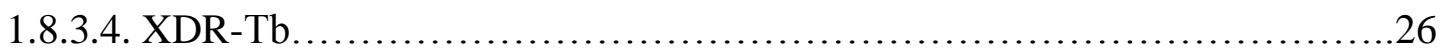

1.8.3.5. XDR-Tb at the global level..........................................27

1.8.3.5.1. XDR-Tb in New Zealand.......................................27

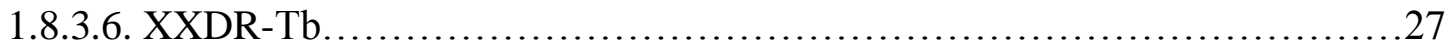

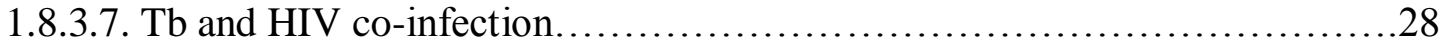

1.8.4. Current pipeline for new anti-Tb drugs.....................................29

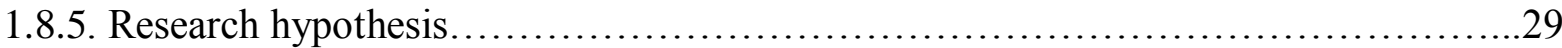

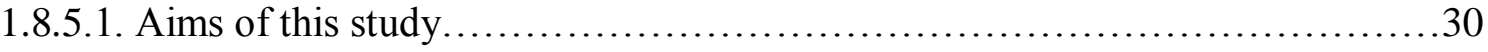

Chapter Two: Materials and Methods.................................................

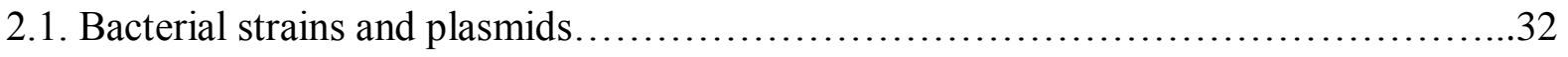

2.1.1. Bacterial strains........................................................ 32

2.1.2. Plasmids......................................................... 33

2.2. Growth media............................................................ 35

2.2.1. Growth requirements for the slow-growers................................ 35

2.2.1.1. Media supplements................................................ 35 
2.2.1.3. Starvation media.................................................... 38

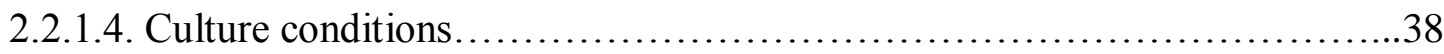

2.2.1.5. Freezer stocks................................................... 39

2.2.2. Mycobacterium smegmatis................................................ 39

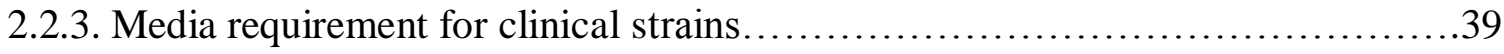

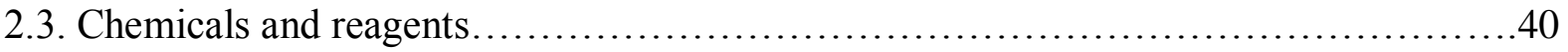

2.4. Enzymes.................................................................. 40

2.5. Oligonucleotides.......................................................40

2.5.1. Primer sequences................................................... 41

2.6. Compound libraries.......................................................... 42

2.6.1. Chemical compound libraries.......................................... 42

2.6.2. Plate map for high throughput screening...................................44

2.6.3. Individual compounds.................................................45

2.6.3.1. NIH compound repository .........................................45

2.6.3.2. 96-well plates for assays..........................................46

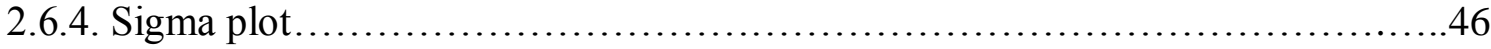

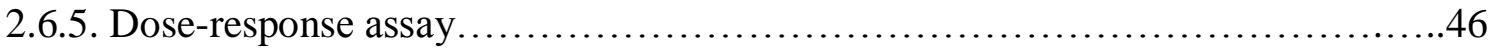

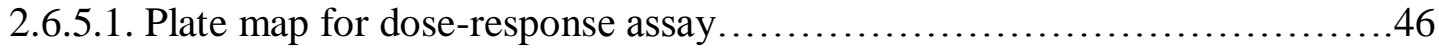

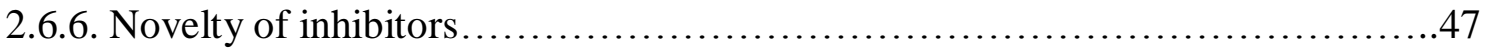

2.6.7. Import of anti-HIV RNase H compounds..............................47

2.6.8. Structure-activity relationships....................................... 47

2.6.8.1. Lipinski’s Rules..................................................48

2.7. Methods............................................................. 49

2.7.1. Tuberculosis screening models........................................49

2.7.2. Growth inhibition assays for mycobacteria...............................49

2.7.2.1. Bacteriostatic assay for $M$. bovis BCG..............................49 
2.7.2.1.1. Bacteriostatic assay for M. tuberculosis

2.7.2.2. Bacteriostatic assay for $M$. smegmatis.............................51

2.7.2.3. Bacteriostatic assay for clinical strains..............................52

2.7.2.4. Colony forming units for mycobacteria.............................52

2.7.2.4.1. CFUs for $M$. tuberculosis..................................5

2.7.2.4.2. CFUs for M. smegmatis......................................53

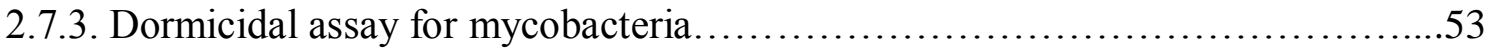

2.7.4. Resazurin reduction assay ............................................... 54

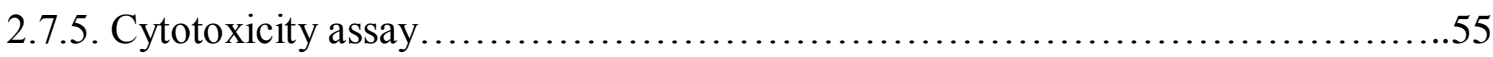

2.7.5.1. J774.A1 cell line................................................55

2.7.5.2. HL-60 cell line.................................................57

2.7.6. Cloning of mycobacterial RNase H into E. coli...............................60

2.7.7. Ligation of inserts into vector...........................................65

2.7.8. Identification of recombinant clones.....................................67

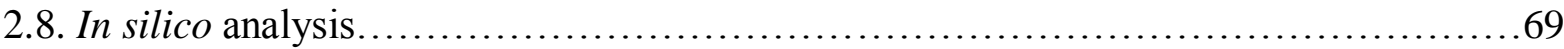

Chapter Three: High Throughput Screening of Chemical Libraries.....................70

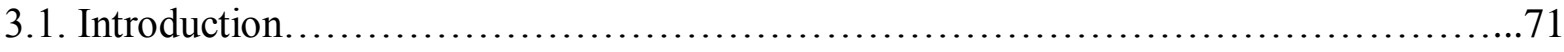

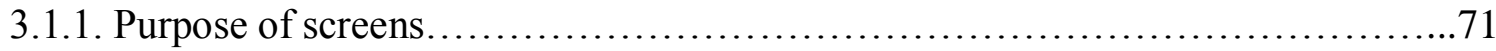

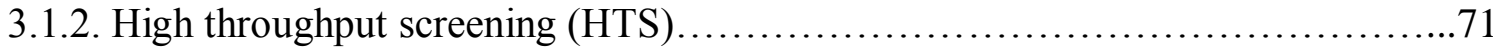

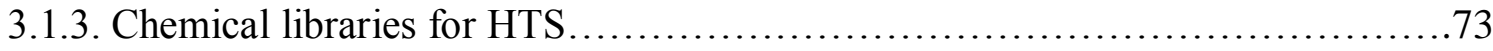

3.1.4. Liquid-handling robot system......................................... 74

3.1.5. Objectives...................................................... 74

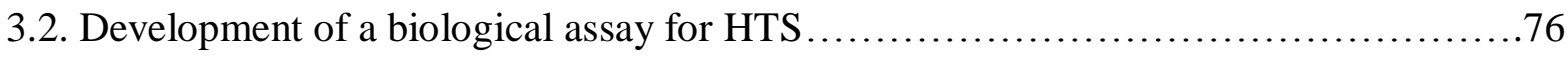

3.2.1. Growth inhibition assay.............................................. 76

3.2.2. Reporter systems................................................ 77

3.2.3. Optimization of a fluorescence-based assay................................ 80 


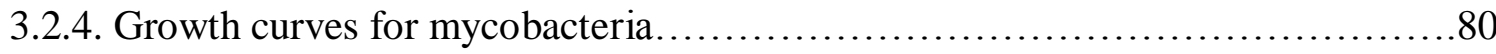

3.2.5. Reliability of a bacteriostatic assay ...................................... 81

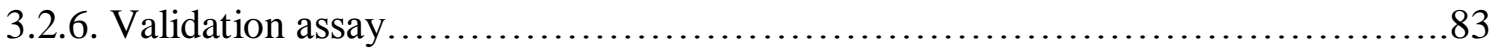

3.2.7. Effect of DMSO on M. bovis BCG growth................................. 84

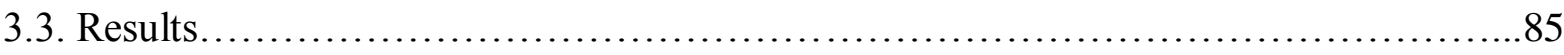

3.3.1. Results for compound libraries......................................... 85

3.3.1.1. Hits from the LOPAC library ...................................... 85

3.3.1.1.4. The selection criteria for hits..................................... 94

3.3.1.2. The Spectrum Collection......................................... 95

3.3.1.3. The NIH library................................................ 100

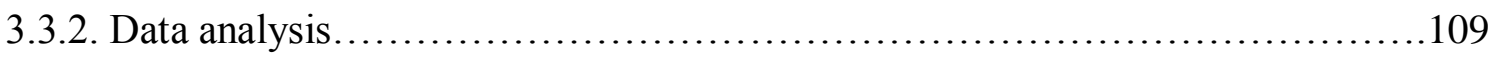

3.3.3. Criteria for promiscuous compounds................................... 113

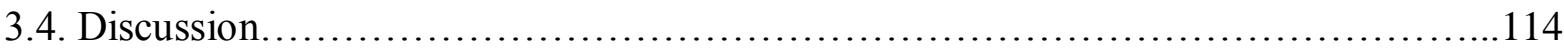

Chapter Four: Tuberculosis Models Comparison...................................119

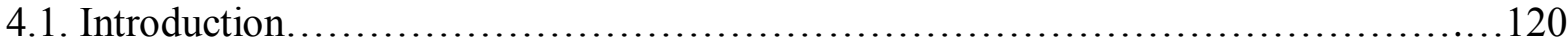

4.1.1. In vitro models for tuberculosis drug discovery ............................ 120

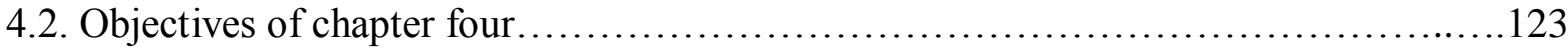

4.3. Comparative genomic analysis............................................ 124

4.3.1. M. tuberculosis genome........................................... 124

4.3.2. Controversy with respect to $M$. smegmatis use............................ 127

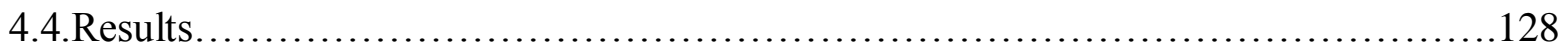

4.4.1. Results from the library screens..................................... 128

4.4.2. Comparison of hits across each $\mathrm{Tb}$ model.............................. 128

4.4.3. Validation of mycobacterial inhibitors.................................. 130

4.4.4. LOPAC screens.................................................. 130

4.4.5. Spectrum Collection............................................... 134 


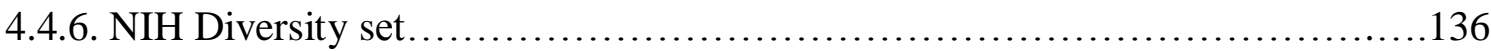

4.4.6.1. M. tuberculosis specific inhibitors......................................137

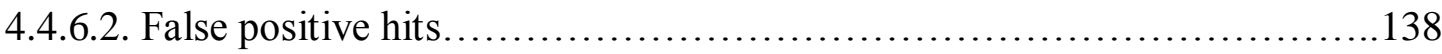

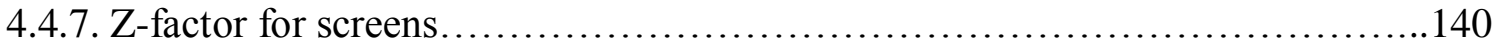

4.4.8. Genome comparison of three $\mathrm{Tb}$ models........................................ 140

4.4.9. Novel anti-M. tuberculosis inhibitor, NSC402959 .............................142

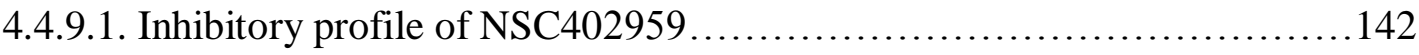

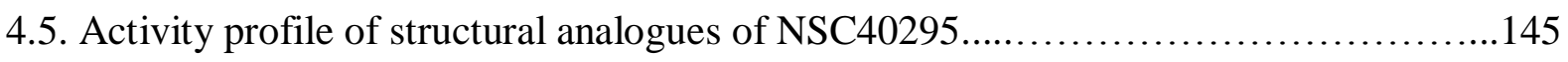

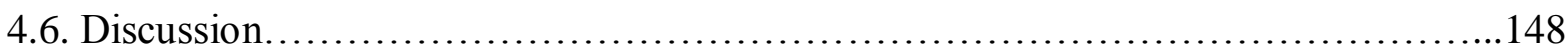

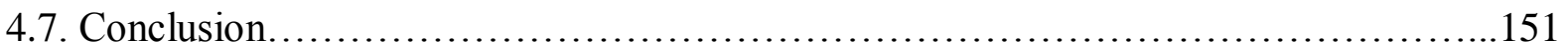

Chapter Five: RNase H as a Target for Dual Inhibitors of Tb and HIV..................152

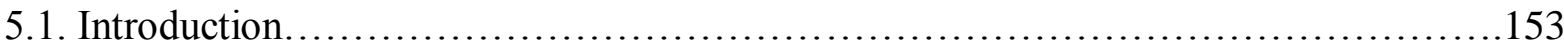

5.1.1. Tuberculosis and HIV - the two pandemics.................................153

5.1.1.1. HIV/Tb, a lethal combination........................................153

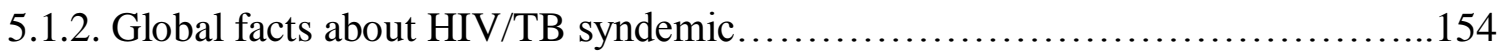

5.2. Demand for the amendment to the current HIV therapeutic regimen..................154

5.2.1. Current anti-Tb therapy and associated problems..............................155

5.2.2. Current anti-HIV therapy and associated problems.............................155

5.2.3. Need for the development of a dual therapy .................................156

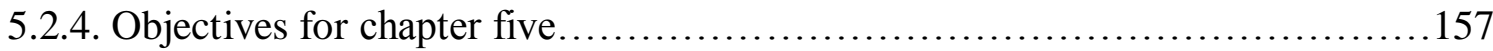

5.3. New strategy of a dual inhibitor of M. tuberculosis and HIV ........................157

5.3.1. Definition of a dual inhibitor..............................................157

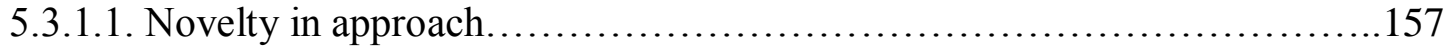

5.3.2. RNase $\mathrm{H}$ as a drug target in M. tuberculosis and HIV ............................158 
5.3.3. Biochemical assay to detect RNase H enzyme activity 159

5.3.3.1. Principle of the enzyme inhibition assay. 159

5.3.4. Criteria for the selection of dual inhibitors 161

5.4. Results. .161

5.4.1. Anti-HIV RNase H compounds 161

5.4.2. In vitro activity of HIV RNase $\mathrm{H}$ inhibitors against $M$. tuberculosis.... 162

5.4.3. Activity of HIV RNase H Inhibitors against $M$. tuberculosis RNase HI. 165

5.4.4. Results for cytotoxicity against human cell line.... .166

5.4.5. Inhibitory profile of RNase H compounds against clinical strains. 168

5.4.6. Cloning of RNase H proteins from M. tuberculosis in E. coli BL21 (DE3).......170

5.4.6.1. Expression of Rv2228c and Rv2902c in E. coli shuttle vector, pET28a.....170

5.5. Molecular docking simulations 172

5.6. Discussion 176

5.7. Structure-activity relationship study. .181

5.8. SAR of RNase H inhibitors 183

5.8.1. Comparison of HIV and M. tuberculosis Inhibitors 183

5.8.1.2. Anti-HIV RNase H inhibitors that do not inhibit human enzyme 185

5.8.2. Effect of hydroxyl groups in mycobacterial inhibitors 189

5.8.3. Description of new inhibitors of M. tuberculosis 190

5.9. New anti-Tb clinical drug pipeline

5.10. Conclusion.

Chapter Six: Summary and Future Directions 196

6.1. Summary of the thesis. 197

6.2. Scientific contributions of this study...... 198 
6.3. Future prospects of this research.......................................... 199

6.4. Possible problems with successful drug development............................202

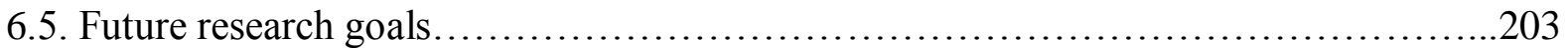

References..........................................................................204

Appendices..............................................................................251

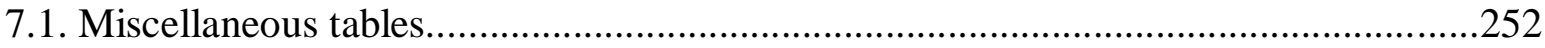

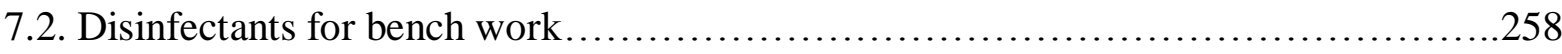

7.2.1. Culture purity for mycobacteria......................................... 258

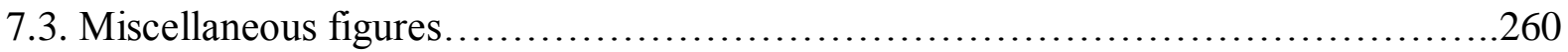




\section{List of Figures}

Figure 1.1: Chest X-ray of a patient with pulmonary Tb..............................4

Figure 1.2: Stages of $M$. tuberculosis infection.....................................6

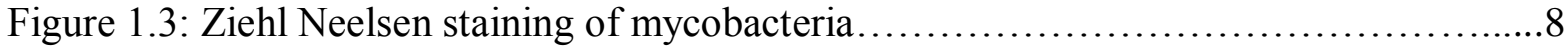

Figure 1.4: Number of Tb reported annually........................................13

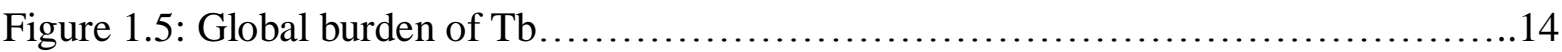

Figure 1.6: Pathology of Tb..................................................... 16

Figure 1.7: Worldwide distribution of MDR-Tb among new Tb cases...................18

Figure 1.8: Current panel of standard anti-Tb Drugs................................. 21

Figure 2.1: Chemical libraries used for HTS......................................44

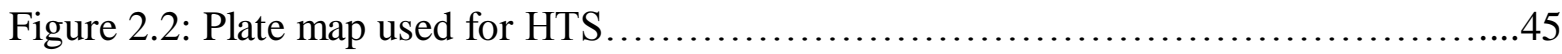

Figure 2.3: Biochemical reduction of resazurin sodium salt into resorufin.................54

Figure 2.4: Plate map for mammalian cell cytotoxicity assays..........................57

Figure 2.5: Improved neubauer chamber.......................................... 59

Figure 3.1: A schematic flow diagram of main research steps of this study.............75

Figure 3.2: Fluorescently labeled M. bovis BCG Pasteur strain 1173P2...................77

Figure 3.3: Time point growth curve for slow-growing mycobacteria....................81

Figure 3.4: Percentage $M$. bovis BCG growth in DMSO................................ 84

Figure 3.5: Distribution of compounds and range of drug targets in LOPAC ...............86

Figure 3.6: Scatter plot for LOPAC screen against $M$. bovis BCG......................87

Figure 3.7: Hits from two independent triplicate Spectrum screens against $M$. bovis BCG....96

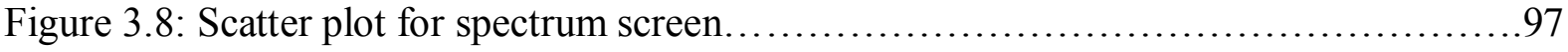

Figure 3.9: Hits from the first three spectrum screens against $M$. bovis BCG and $M$. tuberculosis 100

Figure 3.10: Scatter plot for NIH screen against M. bovis BCG........................101

Figure 3.11: Flow chart for NIH chemical library screen............................ 102

Figure 3.12: Dose-response of NSC402959 against $M$. tuberculosis....................105

Figure 3.13: Activity of NSC15381 against clinical strains............................106 
Figure 3.14: Cytotoxicity of NIH compounds against HL-60 cell line 107

Figure 3.15: A diagram of an HTS 96-well plate. 109

Figure 3.16: Correlation of LOPAC screens against $M$. bovis BCG and $M$. tuberculosis....111

Figure 3.17: Hits in common between M. bovis BCG and M. tuberculosis....

Figure 3.18: Chemical structure of sappanone A....................................116

Figure 4.1: In vitro Tb models for drug screening.................................122

Figure 4.2: Allocated funding for Tb research and development in $2010 \ldots \ldots \ldots \ldots \ldots \ldots \ldots \ldots$

Figure 4.3: The genome of M. tuberculosis H37Rv..................................127

Figure 4.4: Venn diagram for LOPAC library....................................131

Figure 4.5: Dose-response curve of APDC against mycobacteria........................133

Figure 4.6: Dose-response curve of TETD against the mycobacteria.....................133

Figure 4.7: Venn diagrams for spectrum library.................................. 135

Figure 4.8: Venn diagrams for the NIH Diversity set............................... 136

Figure 4.9: Chemical structures for NSC168466, NSC168467 and NSC168468...........137

Figure 4.10: Genome comparison of the mycobacterial species.......................141

Figure 4.11: Anti-Tb activity of NSC402959 against $M$. tuberculosis H37Ra under C-......143

Figure 4.12: Growth inhibitory activity of NSC402959 and rifampicin in M. tuberculosis H37Rv................................................................... 144

Figure 4.13: Cytotoxicity of NSC402959 against HL-60 cell line compared to rifampicin..144 Figure 4.14: Chemical structure of NSC15381, NSC603988 and NSC402959 .............146

Figure 4.15: Chemical structures mycobacterial compounds..........................147

Figure 5.1: RNase $H$ activity required for DNA replication in $M$. tuberculosis and HIV ..........................................................................159

Figure 5.2: Principle of the RNase H assay.......................................... 160

Figure 5.3: Dose-response curve for HIV RNase H inhibitor NSC133457 against

M. tuberculosis.... 166

Figure 5.4: Activity of NSC133457 against the human cell line, HL-60 ...................167

Figure 5.5: Chemical structure of NSC133457 ..................................... 168 
Figure 5.6: Cloning steps for Rv2228c and Rv2902c from M. tuberculosis in the E. coli strains

Figure 5.7: Amplification of M. tuberculosis genes encoding RNase proteins, Rv2228c and

Rv2902

Figure 5.8: Restriction digestion of Rv2228c performed by Bam HI

Figure 5.9: Confirmation of ligation of Rv2228c into pET28a (+)

Figure 5.10: Docking of HIV RNase H inhibitors on M. tuberculosis RNase HI

Figure 5.11: Comparison of similarities between Rv2228/N and its homologues

Figure 5.12: Chemical structure of NSC143101 ................................... 180

Figure 5.13: Structures of anti-Tb drugs............................................

Figure 5.14: Structural comparison of acylhydrazone anti-HIV inhibitors with the $M$. tuberculosis inhibitors identified in this study....

Figure 5.15: Structural comparison of acylhydrazone derivative and NSC130796

Figure 5.16: Chemical structures of NSC45382, NSC83217 and NSC112200

Figure 5.17: SAR of RNase H inhibitors identified in this study with previously reported compounds

Figure 5.18: Structural similarity between an HIV RNase H inhibitor, illimaquinone and

Figure 5.19: Chemical structures of NSC14543, NSC20410 and NSC51535..............189

Figure 5.20: New anti-Tb drugs in the clinical pipeline

Figure 6.1: Future prospects for the development of an inter-kingdom, single-target RNase HI inhibitor. 


\section{List of Tables}

Table 1.1: M. tuberculosis infection and pulmonary tuberculosis disease.................4

Table 1.2: Standard anti-Tb drugs and year of discovery .............................. 21

Table 1.3: Standard anti-Tb drugs and mechanisms of action...........................22

Table 1.4: Genetic basis of drug resistance in $M$. tuberculosis.........................25

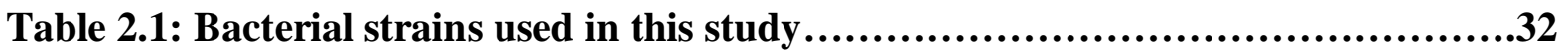

Table 2.2: Bacterial plasmids used in this study.................................... 34

Table 2.3: Mammalian cell lines used in this study....................................

Table 2.4: Primers used in this work.............................................. 41

Table 3.1: Available reporter assays for HTS........................................79

Table 3.2: Comparison of MIC values between M. bovis BCG and M. tuberculosis H37Rv..83

Table 3.3: Hits identified from LOPAC screen against M. bovis BCG and M. tuberculosis...89

Table 3.4: Validated hits from LOPAC against $M$. tuberculosis.......................91

Table 3.5: Validated Hits from spectrum against $M$. tuberculosis.........................98

Table 3.6: Validated M. tuberculosis inhibitors from the NIH library.......................104

Table 3.7: Anti-Tb activity of Native New Zealand plant extracts...................... 108

Table 3.8: Comparison of library hits against BCG and M. tuberculosis..................112

Table 4.1: Validated mycobacterial inhibitors from the LOPAC library................132

Table 4.2: Validated compounds with anti-mycobacterial activity from the NIH library....139

Table 5.1: Activity profile of RNase HI inhibitors..................................163

Table 5.2: Anti-mycobacterial efficacy and toxicodynamics for RNase HI inhibitors of

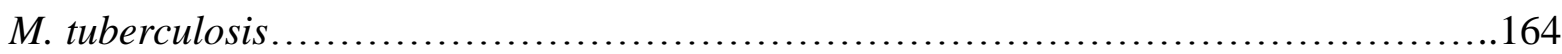

Table 5.3: Activity profile of RNase HI inhibitors against clinical strains.................169

Table 5.4: Binding scores for interaction of inhibitors with M. tuberculosis RNase HI...173 


\section{List of Abbreviations}

\begin{tabular}{|c|c|}
\hline$\mu \mathrm{F}$ & microfarad \\
\hline$\mu \mathrm{L}$ & microliter \\
\hline AFB & Acid Fast Bacilli \\
\hline AIDS & Acquired Immunodeficiency Syndrome \\
\hline $\mathrm{Amp}^{\mathrm{r}}$ & Refers to a resistance gene for Ampicilin \\
\hline AT & Adenine-thymine \\
\hline ATCC & American Type Culture Collection \\
\hline BCG & Bacillus Calmette-Guérin \\
\hline bp & base pairs \\
\hline BLAST & Basic Local Alignment Search Tool \\
\hline BSL & Bio Safety Level \\
\hline C- & Carbon starvation \\
\hline CAP & Capreomycin \\
\hline CDC & Centres for Disease Control and Prevention \\
\hline CFP & Culture Filtrate Protein \\
\hline $\mathrm{CFU}$ & Colony Forming Unit \\
\hline CIP & Ciprofloxacin \\
\hline Conc. & Concentration \\
\hline D-Cs & D-Cycloserine \\
\hline $\mathrm{ddH}_{2} \mathrm{O}$ & Double distilled water \\
\hline DMSO & di-Methyl sulfoxide \\
\hline
\end{tabular}




\begin{tabular}{|c|c|}
\hline DNA & Deoxyribonucleic Acid \\
\hline dNTPs & deoxynucleotide triphosphates \\
\hline DOTS & Directly Observed Treatment Short-Course \\
\hline DTP & Developmental Therapeutics Program \\
\hline E. coli & Escherichia coli \\
\hline EDTA & Ethylenediaminetetraacetic acid \\
\hline ESAT & Early Secreted Antigenic Target \\
\hline ESR & Institute of Environmental Science and Research Limited \\
\hline ETH & Ethambutol \\
\hline ETM & Ethionamide \\
\hline FDA & Food and Drug Administration \\
\hline GC & Guanine-Cytosine \\
\hline GEN & Gentamicin \\
\hline GFP & Green Fluorescent Protein \\
\hline HIV & Human Immunodeficiency Virus \\
\hline HTS & High-Throughput Screen \\
\hline $\mathrm{IC}_{50}$ & $50 \%$ Inhibitory Concentration \\
\hline ID & Infectious Dose \\
\hline Inc. & Incorporation \\
\hline INH & Isoniazid \\
\hline JHU & Johns Hopkins University \\
\hline $\operatorname{kan}^{r}$ & Kanamycin resistant \\
\hline kan+ & with kanamycin \\
\hline
\end{tabular}


$\mathrm{kb}$

kilo base

LB

Luria-Bertani

LOPAC Library of Pharmacologically Active Compounds

LTBI Latent Tuberculosis Infection

MAF Ministry of Agriculture and Forestry, New Zealand

M. africanum Mycobacterium africanum

MCS Multiple Cloning Sites

MDG Millennium Development Goals

MDR Multi-drug Resistance

MF Molecular Formula

$\mathrm{MgCl}_{2} \quad$ Magnesium Chloride

MIC Minimum Inhibitory Concentration

MIMR Malaghan Institute for Medical Research

mm Millimeter

M. leprae Mycobacterium leprae

M. smegmatis Mycobacterium smegmatis

M. tuberculosis Mycobacterium tuberculosis

Mtb Mycobacterium tuberculosis

MTBC Mycobacterium tuberculosis Complex

MW Molecular Weight

NCI National Cancer Institute

NEB New England Bio Labs

NIAID National Institute of Allergy and Infectious Diseases 


\begin{tabular}{|c|c|}
\hline $\mathrm{NIH}$ & National Institutes of Health \\
\hline NIWA & National Institute of Water and Atmospheric Research \\
\hline $\mathrm{nm}$ & nanometer \\
\hline NZ & New Zealand \\
\hline OADC & Oleic acid Albumin Dextrose Complex \\
\hline OD & Optical Density \\
\hline $\mathrm{OD}_{600}$ & Optical Density at $600 \mathrm{~nm}$ \\
\hline ORF & Open Reading Frame \\
\hline PBS & Phosphate Buffered Saline \\
\hline PC2 & Physical Containment Level Two \\
\hline PCR & Polymerase Chain Reaction \\
\hline pmol & Picomole (1.0E-12) \\
\hline PYZ & Pyrazinamide \\
\hline RD & Region of Difference \\
\hline RFU & Relative Fluorescence Unit \\
\hline RIF & Rifampicin \\
\hline RNase H & Ribonuclease $\mathrm{H}$ \\
\hline Rpm & Revolutions per minute \\
\hline RT & Reverse Transcriptase \\
\hline SAR & Structure Activity Relationship \\
\hline SD & Standard Deviation \\
\hline STP & Streptomycin \\
\hline
\end{tabular}

TAACF Tuberculosis Antimicrobial Acquisition and Coordinating Facility 
TAE

$\mathrm{Tb}$

TET

USA

V

VUW

WHO

WT

XDR

$\Omega$

ohms

volts
Tris Acetate EDTA

Tuberculosis

Tetracycline

United States of America

Victoria University of Wellington

World Health Organisation

Wild type

Extensive-drug Resistance 


\section{List of Publications}

- Altaf. M, Miller. C. H, Bellows. D. S and O'Toole. R (2010). Evaluation of the Mycobacterium smegmatis and BCG models for the discovery of Mycobacterium tuberculosis inhibitors. Tuberculosis. 90(6): p. 333-337.

- Earl. E, Altaf. M, Murikoli. R. V, Swift. S and O'Toole. R (2010). Native New Zealand plants with inhibitory activity towards Mycobacterium tuberculosis. BMC Complementary and Alternative Medicine. 10(1): p. 25. 
Chapter 1

General Introduction to Tuberculosis Biology and Drug Discovery 


\section{General Introduction to Tuberculosis Biology And Drug Discovery}

\subsection{Tuberculosis}

Tuberculosis $(\mathrm{Tb})$ is a debilitating and highly contagious disease that primarily affects the lungs [1]. It was declared a global health emergency in 1993 by the World Health Organization (WHO) when approximately 8 million $\mathrm{Tb}$ cases were estimated and 1.3-1.6 million deaths occurred from the disease each year [2]. $\mathrm{Tb}$ is still a major public health concern as it is a leading bacterial cause of death worldwide [3]. A record number of incident cases of Tb, 8.8 million, were estimated in 2010 alone [4]. Pulmonary $\mathrm{Tb}$ is the most prevalent form of the disease and presents various signs and symptoms indicating an active state of the disease such as cough (could be non-productive at early stages), low-grade fever, night sweats, loss of appetite, fatigue and consistent weight loss [1, 5]. Unlike immunocompromised individuals, Tb occasionally can be extra-pulmonary affecting other parts of the body such as miliary $\mathrm{Tb}$ (disseminated disease), $\mathrm{Tb}$ meningitis or an oral $\mathrm{Tb}[1,6]$. Miliary $\mathrm{Tb}$ is a rare form of the disease that can complicate the primary infection [7]. It produces tiny lesions involving vital organs including lungs and can cause pulmonary failure [8-10]. Tb has consistently been a public health problem for decades (Figure 1.4), and therefore global $\mathrm{Tb}$ control strategies have to be thoroughly implemented in order to achieve effective control of the disease [11-12].

\subsection{Causative Agent of $\mathbf{T b}$}

Mycobacterium tuberculosis (M. tuberculosis or Mtb) is the primary cause of $\mathrm{Tb}$ in humans and is also termed as tubercle bacilli [13]. It was first discovered by Robert Koch in 1882, who was awarded with a Noble prize for his remarkable discovery [14-16]. Cole et al. were the first to perform the complete genome sequencing of M. tuberculosis $\mathrm{H} 37 \mathrm{Rv}$ in 1998 [17] 
which was later revised in 2002 [18]. It has a genome size of $4.4 \mathrm{~kb}\left(4 \times 10^{6}\right.$ base pairs) and 4056 genes with 3995 predicted proteins [18]. M. tuberculosis is an extremely slow-growing mycobacterial species, with a generation time of 18-24 hours [19]. Infection caused by $M$. tuberculosis is air-borne and spreads through an aerosol contamination [20-21]. The infectious dose (ID) for M. tuberculosis is very low and $\mathrm{ID}_{50}$ has been estimated to be $<10$ bacilli in humans [22]. Predominant contagious factors are sneezing, coughing or spitting by infected patients, thereby releasing tubercle bacilli into the air. Therefore, healthy people are vulnerable to catching $\mathrm{Tb}$ infection through air-borne droplets containing viable bacilli [23]. Since airborne infection is highly contagious, a high level of supervision and a separate facility is required to contain this infectious (BSL3) pathogen within the transition facility [24]. Physical containment level three (PC3) is required to work with these pathogenic species [25]. Specific HEPA filters (High Efficiency Particulate Air) have to be in place in the Biological Safety Cabinets for personnel safety as well as for the safety of the environment [26].

\subsubsection{Comparison of Latent Tb Infection and Active Disease}

Despite the presence of $M$. tuberculosis in a host, it may not produce disease symptoms due to an efficient host immune system [27]. In this case M. tuberculosis becomes non-replicative in vivo and the condition is termed as "latent tuberculosis infection" (LTBI) (section 1.6); whereas $\mathrm{Tb}$ disease refers to a highly contagious situation, in which disease signs and symptoms are produced causing damage to lungs (Figure 1.1) [5]. Apart from some clinical similarities, there are a number of differences between $M$. tuberculosis infection and $\mathrm{Tb}$ disease (Table 1.1). It is important to differentiate between these two conditions for proper clinical evaluation and case diagnosis (section 1.8.1). 
Table 1.1: $M$. tuberculosis infection and pulmonary Tb disease

\begin{tabular}{ll}
\hline M. tuberculosis Infection & Active Tb Disease \\
\hline M. tuberculosis is present & M. tuberculosis is present \\
Clinical symptoms are not present & ${ }^{1}$ Sign and symptoms are present for Tb \\
Not contagious & Highly contagious \\
Tuberculin skin test is positive & ${ }^{2}$ Tuberculin skin test is positive \\
Sputum smear negative for Zn stain & Sputum smear positive for Zn stain \\
Sputum culture is negative for $M t b$ growth & Sputum culture is positive for Mtb growth \\
Chest X-ray appears normal & ${ }^{3}$ Chest X-ray shows lesions and lung damage \\
9-month isoniazid monotherapy [28] & ${ }^{4}$ Chemotherapy for drug-susceptible Tb
\end{tabular}

${ }^{1}$ Section 1.1, p2. ${ }^{2}$ Section 1.8.1, p19. ${ }^{3}$ Figure 1.1, p4. ${ }^{4}$ Table 7.1, p252

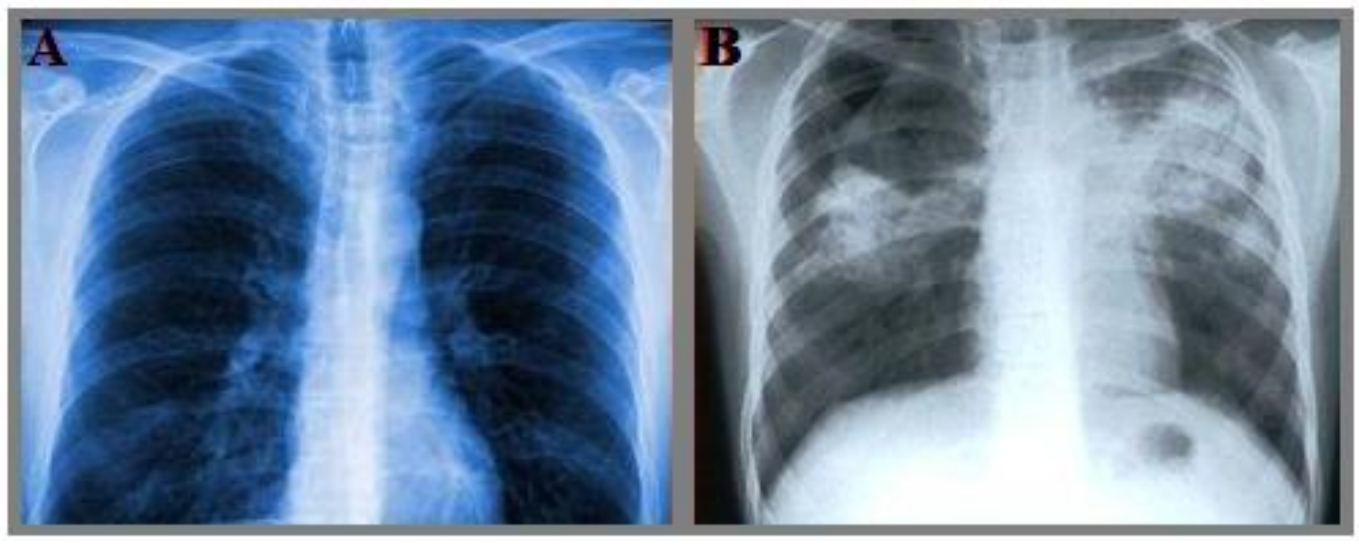

Figure 1.1: Chest X-ray of a patient with pulmonary Tb (A) Normal chest X-ray revealing healthy lungs from an adult male (B) Chest X-ray showing lung damage due to an active Tb disease. Figures were adapted from Science Photo Library [29] and Carolyn et al. [30], respectively. 


\subsection{Mycobacterium tuberculosis Complex}

There are various closely related mycobacterial species which are part of the M. tuberculosis complex (MTBC). Main species of MTBC are M. tuberculosis, Mycobacterium bovis (M. bovis) and Mycobacterium africanum (M. africanum). While they present some differences in their host range, morphology and virulence, they share a high genetic similarity with each other [31].

\subsubsection{Mycobacterium tuberculosis}

M. tuberculosis $(M t b)$ is a member of the MTBC and is known to be a primary cause of human disease. A thick cell wall is one of the main features of M. tuberculosis that differentiates it from other bacterial species. It contains some unique glycolipids that may provide virulence and resistance to antibiotics [32]. It is an extremely slow-growing mycobacterial species compared to all other bacterial groups. M. tuberculosis survives and replicates within host macrophage cells. Despite extensive Tb research, little is known about M. tuberculosis pathogenicity as well as its intra-macrophage replication. M. tuberculosis grown in vitro differs from Mtb isolated from animal models (in vivo) in a number of ways [33]. The pathogenicity of $M$. tuberculosis affects the intensity of $M$. tuberculosis infection and its possible transmission. In order to transmit infection and to establish their pathogenicity, microbial species must leave their respective hosts. Likewise, an exit strategy of M. tuberculosis from the macrophage cells demonstrates one of its virulence factors [34]. M. tuberculosis has evolved ways to evade the host immune system, allowing it to survive within macrophages. It resists the action of reactive oxidative radicals within the these cells, thus demonstrating its pathogenicity and survival tactics within the host [35]. M. tuberculosis is known to cause apoptosis of infected human macrophages before the establishment of 
extracellular persistence and dissemination and uses unique (M. tuberculosis-specific) signals for protein transportation within the host [36]. It is therefore extremely important to know the mechanisms involved in M. tuberculosis persistence and survival within macrophages and how it extends infections to other hosts [37]. Figure 1.2 shows several outcomes after initial mycobacterial infection in the host.

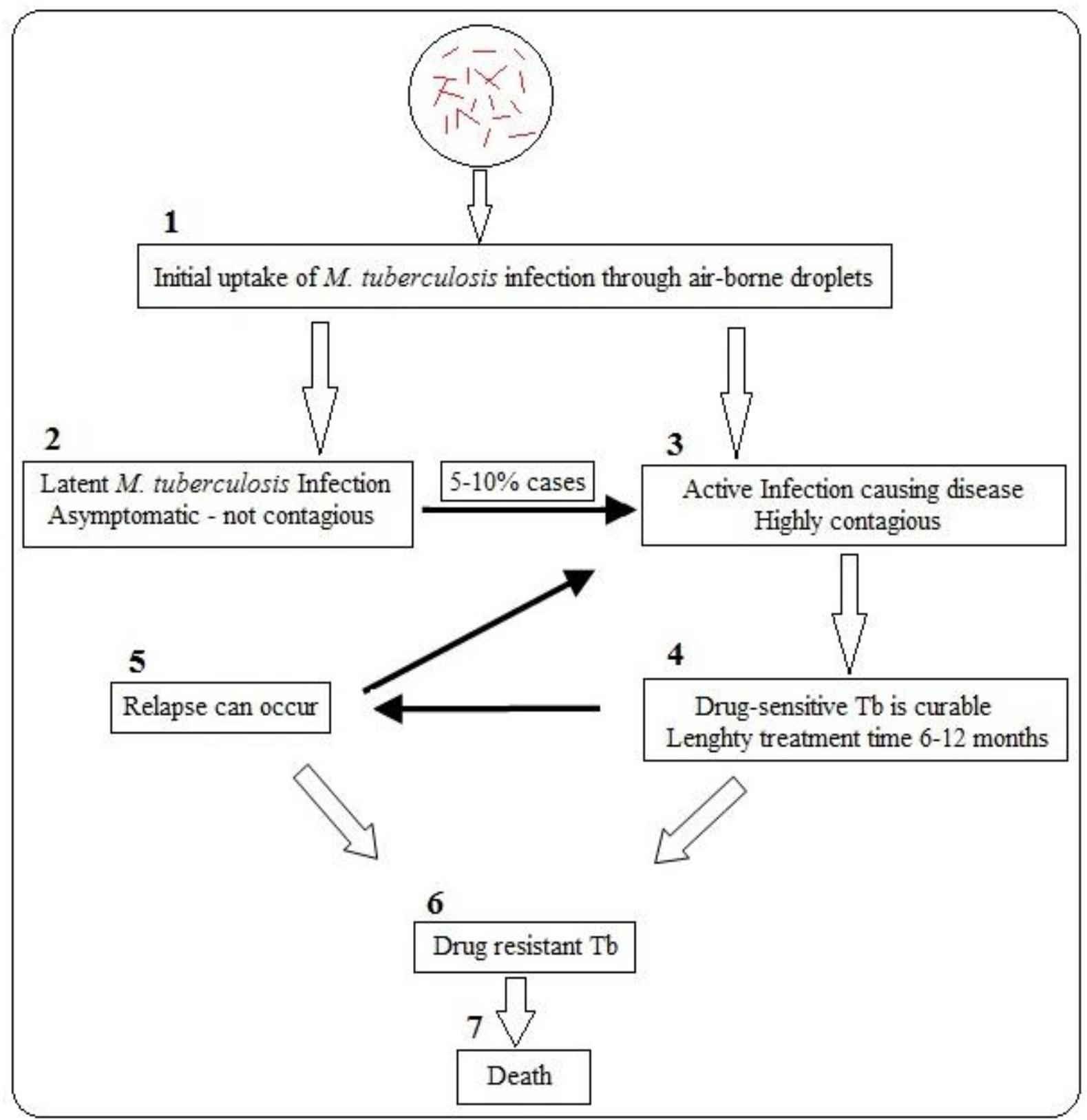

Figure 1.2: Stages of M. tuberculosis infection. (1) Inhalation of M. tuberculosis cells establishes infection in the host. (2) Depending on the number of cells inhaled and competency of the host immune system (section 1.2), 
active state infection cannot occur for several years (3) Or it can be developed into an active Tb disease (4) Chemotherapy is available for drug-sensitive $\mathrm{Tb}$ and is curable (5) At any stage in life, up to $10 \%$ of infections can be reverted and cause disease (6) Due to lack of proper treatment, drug-resistant Tb may develop (7) $M$. tuberculosis does not respond to available chemotherapy in a multiple drug resistant case, which may lead to death.

\subsubsection{Morphology and Staining Properties of Mycobacterium tuberculosis}

M. tuberculosis is an aerobic, non-spore forming and 1-5 $\mu \mathrm{m}$ long slightly-curved rod [22]. The bacilli are non-motile and are gram positive acid fast [38]. Ziehl-Neelsen (Zn) stain, a distinguished staining procedure for mycobacteria that has been considered sensitive for detecting acid-fast bacilli or AFBs [39]. M. tuberculosis cells stain red by taking up the colour of carbol fuchsin and holding it throughout acid decolourization (with acid alcohol), leaving a pink stain (Figure 1.3). Zn staining method was originally developed by Ehrlich in 1882, who described the acid fast properties in mycobacteria [40] and the staining procedure was later modified in 1883 by Ziehl and Neelsen [41-42]. This acid-fastness is due to the amount of mycolic acid present in mycobacterial cell wall along with long-chain fatty acids (Figure 7.3-I) [43]. 


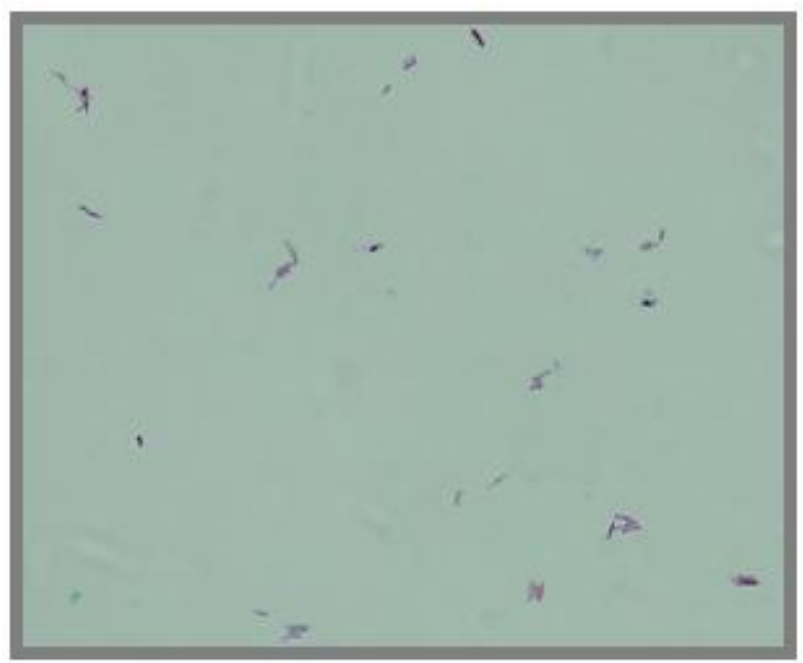

Figure 1.3: Ziehl-Neelsen staining of mycobacteria. M. tuberculosis H37Ra (pink) stained by the modified Ziehl Neelsen staining method.

\subsubsection{Cell Wall of M. tuberculosis}

M. tuberculosis possesses a unique cell wall structure (Figure 7.11) that makes it different from other bacteria in terms of surviving in a range of environmental stress conditions. Unlike other bacterial species, the amount of mycolic acid lipid content is higher in $M$. tuberculosis cell wall [44], and is relatively resistant to some chemical disinfectants [45-47]. However, satisfactory bactericidal efficiency can be achieved by using a $2 \%$ aqueous glutaraldehyde solution at room temperature for 20 minutes [48]. A highly impermeable and thick waxy coat of $M$. tuberculosis has been considered as one of the key factors associated with its pathogenicity [49]. It possesses some unique lipids such as lipoarabinomannan (LAM), phthiocerol dimycocerate and trehalose dimycolate, which are attached to the cell membrane and are thought to be associated with its virulence as well as persistence [50-51]. LAM is linked with $M$. tuberculosis pathogenicity in many ways. For example, in $M$. tuberculosis virulent strains like H37Rv and Erdman strain, LAM serves as a ligand to bind mannose receptors used for virulence-associated intracelluar trafficking [52]. LAM is also 
responsible for inducing tumour necrosis factor (TNF) release from resident macrophages [53].

\subsubsection{Phylogeography of M. tuberculosis}

There are six lineages of M. tuberculosis that are prevalent worldwide. Gagneux et al. have identified the main lineages and the global distribution of $M$. tuberculosis. Since the host immune response is strain-specific, different strains pose different risks with respect to their virulence that influences the host immunity [54-55].

\subsubsection{Mycobacterium bovis}

M. bovis is a member of the MTBC and is closely related to M. tuberculosis [56]. It is responsible for causing bovine $\mathrm{Tb}$ and can affect a range of cattle species and other mammals [57]. M. bovis usually does not cause disease in humans; however, occasionally humans can be affected either by consumption of infected milk or by close contact with an infected animal [58-59]. Moreover, evidence of a zoonotic infection of $M$. bovis in humans has also been documented in the UK [60].

\subsubsection{Mycobacterium bovis BCG}

Mycobacterium bovis Bacillus Calmette-Guérin (M. bovis BCG) is a Tb vaccine strain used in a live-attenuated form to provide immunity from $\mathrm{Tb}$ infection. It was originally derived from $M$. bovis in the $19^{\text {th }}$ century. Two French scientists, Calmette and Guérin prepared this vaccine after 230 passages of $M$. bovis strain in vitro [61]. On continuous passaging, M. bovis lost its virulence and later research confirmed genome differences between avirulent $M$. bovis BCG and virulent $M$. bovis with three large genomic deletions (RD1-RD3) that are responsible for the loss of virulence in BCG [62]. These specific regulatory mutations were 
found to be consistent in various avirulent BCG strains. Reinserting (RD1) gene into BCG induces significant growth and morphological changes making BCG virulent [63] (section 4.3.1.1). M. bovis BCG has been used as a vaccine for Tb since 1921 in the public health system but controversy remains with respect to its use as an effective and complete $\mathrm{Tb}$ vaccine and the differential immune response it generates in different hosts [64-67]. It has also been used for protection against leprosy infections [68-69].

\subsection{Tuberculosis Vaccine}

Cellular immunity is important to protect people against Tb. BCG is the only available vaccine whose efficiency is variable and provides up to $50 \%$ protection for an adult pulmonary disease and about $70-80 \%$ for more severe form of the disease [70-71]. While it has high efficacy against extra pulmonary disease in children [72], it is of no use to adult patients who already have $\mathrm{Tb}$ [73]. Moreover, protection from BCG vaccine is a concern in HIV-infected children who are immuno-compromised [74]. A study by Van der Wel et al shows that M. tuberculosis translocates from phagolysosomes to cytosol for replication, in contrast to the M. bovis BCG strain which does not [75]. This could be due to lack of activation of CD8+ cells and might be one reason why BCG is not effective in adults and immuno-compromised patients with an active disease.

Apart from BCG booster vaccine [76-78], various Tb vaccines are in the clinical trial phase to replace the currently used BCG vaccine for improved efficacy. For instance, a more effective attenuated whole cell mycobacterial vaccine, M. tuberculosis vaccae was tested in HIVinfected individuals, and yielded good outcomes for patients who had prior BCG immunisation [79]. Currently, it is in phase III for clinical evaluation as it seems more promising for developing a cellular-based immunity against $\mathrm{Tb}$. 


\subsubsection{Mycobacterium africanum}

M. africanum is one of the group members of the MTBC and shares a high genetic homology to $M$. tuberculosis [80-81]. It causes human pulmonary $\mathrm{Tb}$ but occasionally can also affect other parts of the body i.e. skin and mucosa [82-83]. Castets et al. first identified $M$. africanum among West-African strains in 1968. It can be further sub-divided on the basis of phenotypic variations into $M$. africanum subtype I (West-African) and subtype II (EastAfrican) [84-85].

\subsubsection{Mycobacterium leprae}

Mycobacterium leprae (M. leprae) is closely related to M. tuberculosis [86]. It causes leprosy and has a prolonged history of afflicting humans [87]. Leprosy primarily affects skin and peripheral nerves [88]. M. leprae infection is mainly transmitted through nasal droplets and its DNA can also be found in healthy individuals [89-90]. M. leprae is resistant to a range of environmental stresses outside the host [91], a complete understanding of $M$. leprae infection, transmission and distribution is important for enhanced vigilance of infection control and disease eradication [92].

\subsection{History of $\mathbf{T b}$}

$\mathrm{Tb}$ has a prolonged history of afflicting human populations, dating back to thousands of years. The earliest evidence of prehistoric $\mathrm{Tb}$ was found in skeletons dated back to 9000 years from a Neolithic Settlement in the Eastern Mediterranean [93]. The disease has been known by several different names in the past. For example, phthisis was known for pulmonary $\mathrm{Tb}$ during the Greek era and was later called as scrofula ( $\mathrm{Tb}$ of the lymph nodes), a unique form of $\mathrm{Tb}$ which affects younger people and children [94]. The term white plague was coined 
around $17^{\text {th }}$ century to describe worldwide adverse effects of pulmonary $\mathrm{Tb}$ [95]. Furthermore, evidence of $\mathrm{Tb}$ was found in the ancient Egyptian mummies responsible for the spinal deformities and bone infections known as pott's disease [96]. Previous evidences do confirm the presence of $\mathrm{Tb}$ and its contagiousness since the era of antiquity [97-98].

\subsection{The Global Burden of $\mathbf{T b}$}

$\mathrm{Tb}$ is a medieval disease that has afflicted mankind for centuries [93], claiming millions of lives [99]. The WHO has estimated about 8.8 million incident Tb cases in 2010 and over a million deaths associated with Tb worldwide [100]. According to the WHO report 2011, the major contribution to the total number of $\mathrm{Tb}$ cases was from the developing world, especially Asia (59\%) and Africa (26\%) [100]. Nearly one third of the world's population is infected with M. tuberculosis [101-102], of which, 5-10\% of latent infections can develop into an active disease at some stage [103]. At least twenty-two high burden Tb countries have been reported, which account for more than $80 \%$ of new $\mathrm{Tb}$ cases [104]. However, global $\mathrm{Tb}$ control efforts aim to not only reduce the mortality rate but also the annual $\mathrm{Tb}$ incidence rate [105]. Despite extensive control efforts, the overall $\mathrm{Tb}$ burden in some countries is not reducing at a constant rate [106]. The 'Stop Tb Partnership' by the WHO and the United Nations (UN), Millennium Development Goals (MDGs) have set their mutual targets for the complete implementation of Directly Observed Therapy Short-Course (section 1.8.2.2.1) for Tb. This is to ensure treatment success, especially in the resource-poor countries and to reduce the overall $\mathrm{Tb}$ burden by $50 \%$ by 2015 as compared to what it was in 1990 [107]. Figure 1.4 depicts the number of new Tb cases reported over the last decade. 


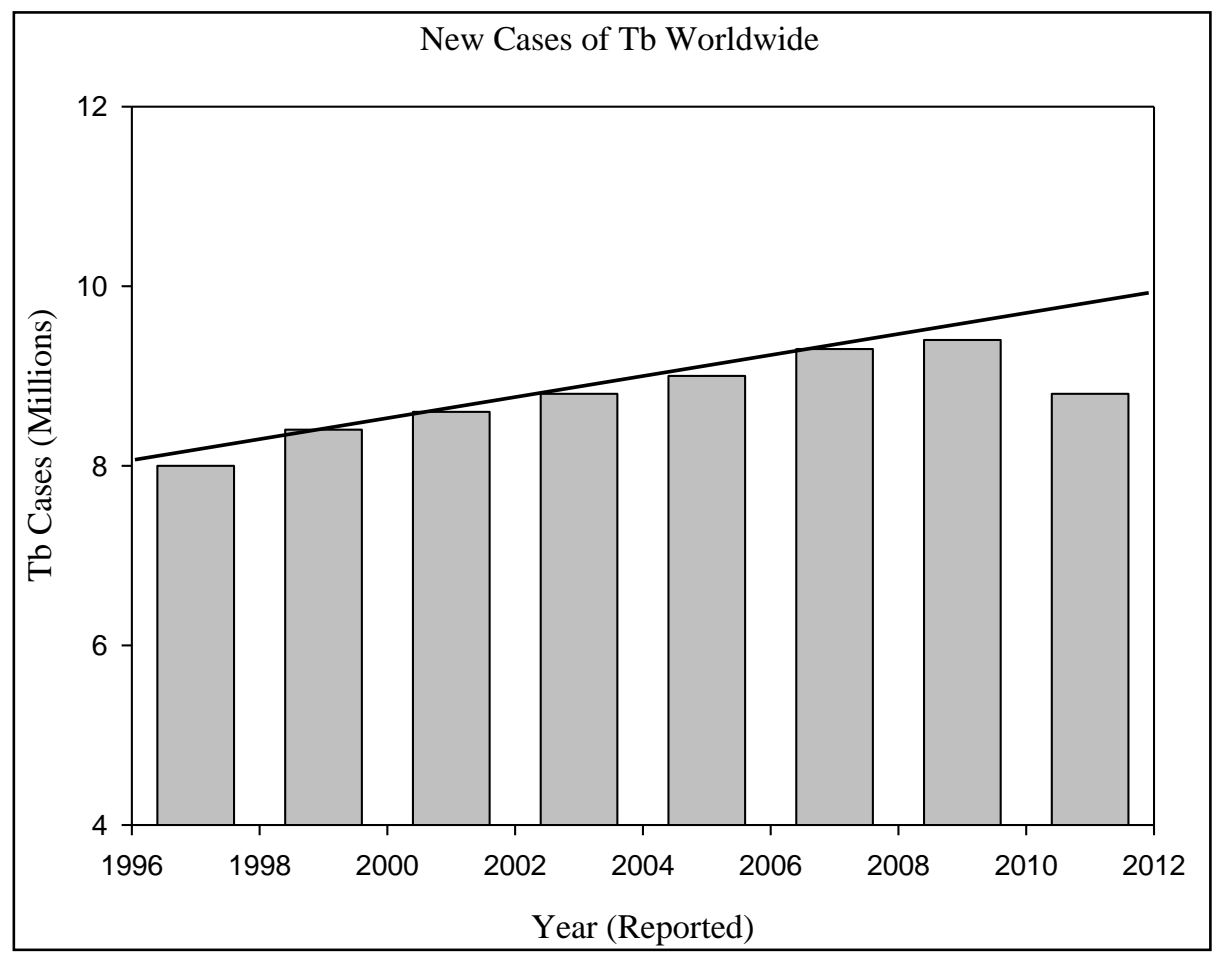

Figure 1.4: Number of Tb cases reported annually. Increase in trend for the annual number of new $\mathrm{Tb}$ cases is obvious over the last decade. While recent reports show that number of new Tb cases have slightly decreased in some countries, drug resistance and Tb/HIV co-infection still pose threats to control efforts. Therefore, more potent drugs are required to make the current $\mathrm{Tb}$ treatment regimen more effective and to cope with the global disease burden and drug resistance problem [108]. 


\section{A. Estimated Tb incidence rates, by country, 2010}

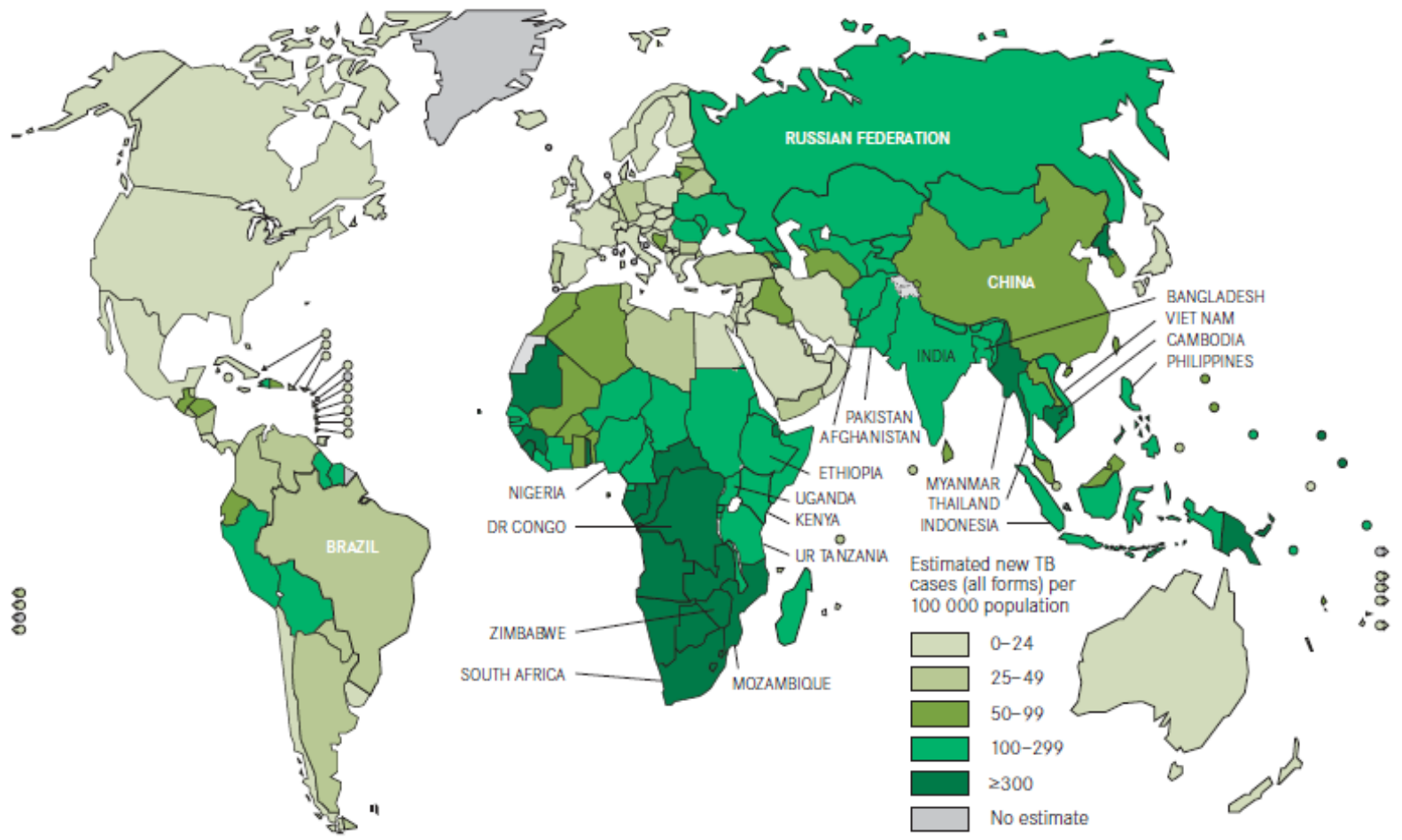

B. Estimated HIV prevalence in new Tb cases, 2010

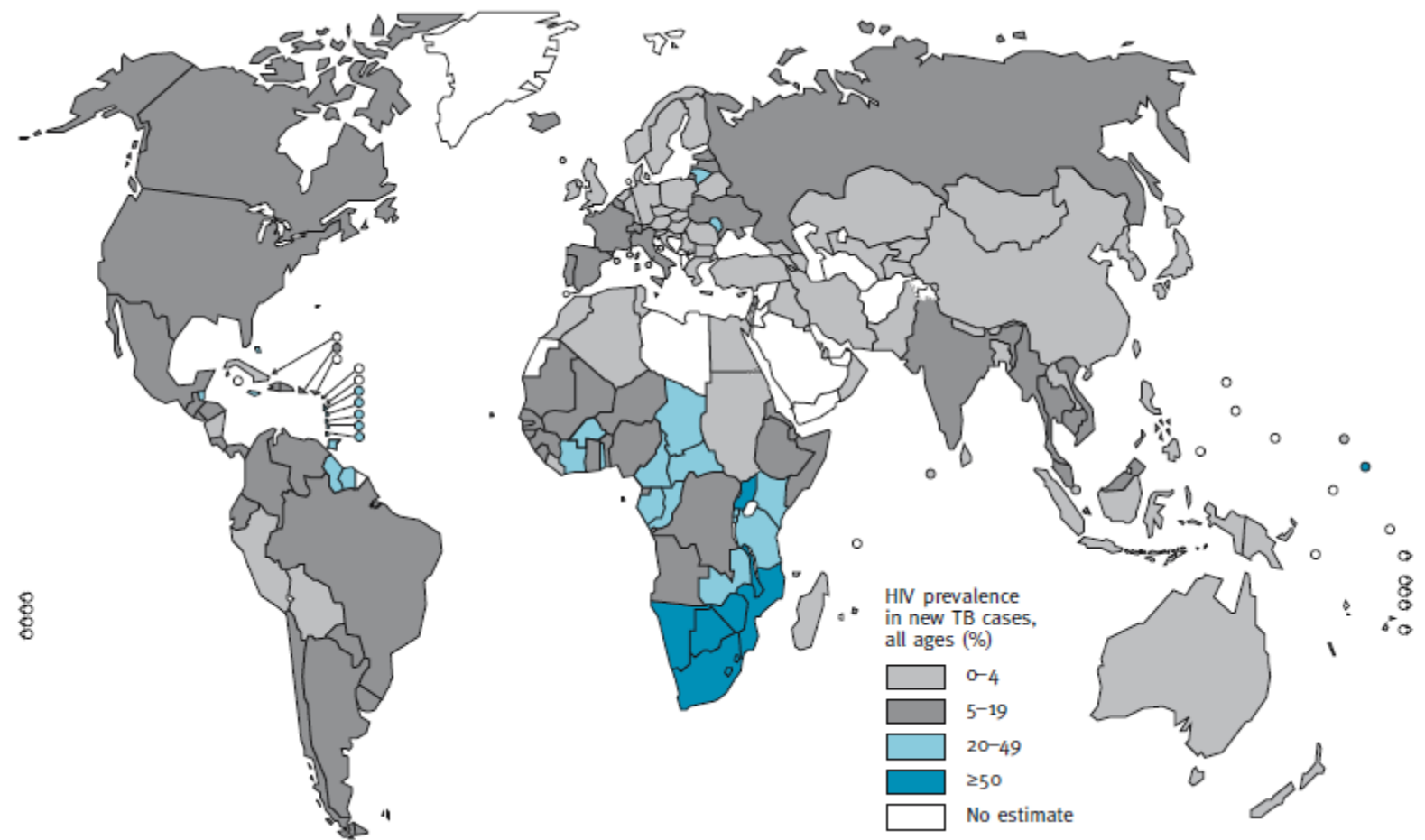

Figure 1.5: Global burden of $\mathbf{T b}$ (A) Global incidence of $\mathrm{Tb}$ in countries with highest impact in Africa, India and China. (B) Impact of HIV in new Tb cases around the world (Reproduced with permission from WHO, Global Tuberculosis Control, report 2010) [104]. 


\subsection{Latent $\mathrm{Tb}$}

Despite harbouring $M$. tuberculosis, people do not usually produce disease symptoms during latent $\mathrm{Tb}$ infections. However, infection can develop into an active $\mathrm{Tb}$ disease at some stage depending on the host immune system [109-110]. One third of the world population is latently infected with $M$. tuberculosis $[102,111]$. It is a recalcitrant pathogen, which can resist host immunity within macrophages, unlike other bacterial species [112-113]. It enters into a dormant state against strong natural host defence and proliferates with a significantly slow growth rate [114]. During host immuno-compromised state, M. tuberculosis can revert from its dormancy into an active disease [115]. The dormant state and slow growth rate of $M$. tuberculosis hinder the process of effective control of the disease [116]. New interventions are required to eliminate latent infections as previously described [117].

\subsubsection{M. tuberculosis Dormancy and Persistence}

M. tuberculosis is believed to maintain two distinct growth phases, i.e. active state (replicative) and non-replicative state, referred to persistence or non-growth condition for longer time period [118]. The term "persisters" was first used for staphylococcus species which survived the initial penicillin therapy [119]. These cells are metabolically inactive but not dead and may provide drug tolerance [120]. The term persistence was later used for the prolonged survival of $M$. tuberculosis within tubercle lesions despite chemotherapy [121]. Various in vitro models have been previously used to study mycobacterial persistence by inducing hypoxia under oxygen-depletion conditions [122-126]. Hypoxia is one of the main environmental stresses that drives $M$. tuberculosis population into dormancy [127]. It leads the non-replicative $M$. tuberculosis phenotypes towards drug tolerance, which could be one of the factors responsible for prolonging $\mathrm{Tb}$ treatment [128]. It is hard to eliminate dormant mycobacteria that despite several months of chemotherapy, most drugs cannot easily target 
non-replicating bacilli. These persister cells can revive later in life and develop into an active disease [129]. Therefore, control of M. tuberculosis infections at a global scale is perhaps more important than its eradication [130]. Nitroimidazole compounds (PA824) are the first class of drugs (currently in clinical trials) that also possess activity against persistent $M$. tuberculosis cells [131]. Figure 1.6 presents pathology of $M$. tuberculosis infections.

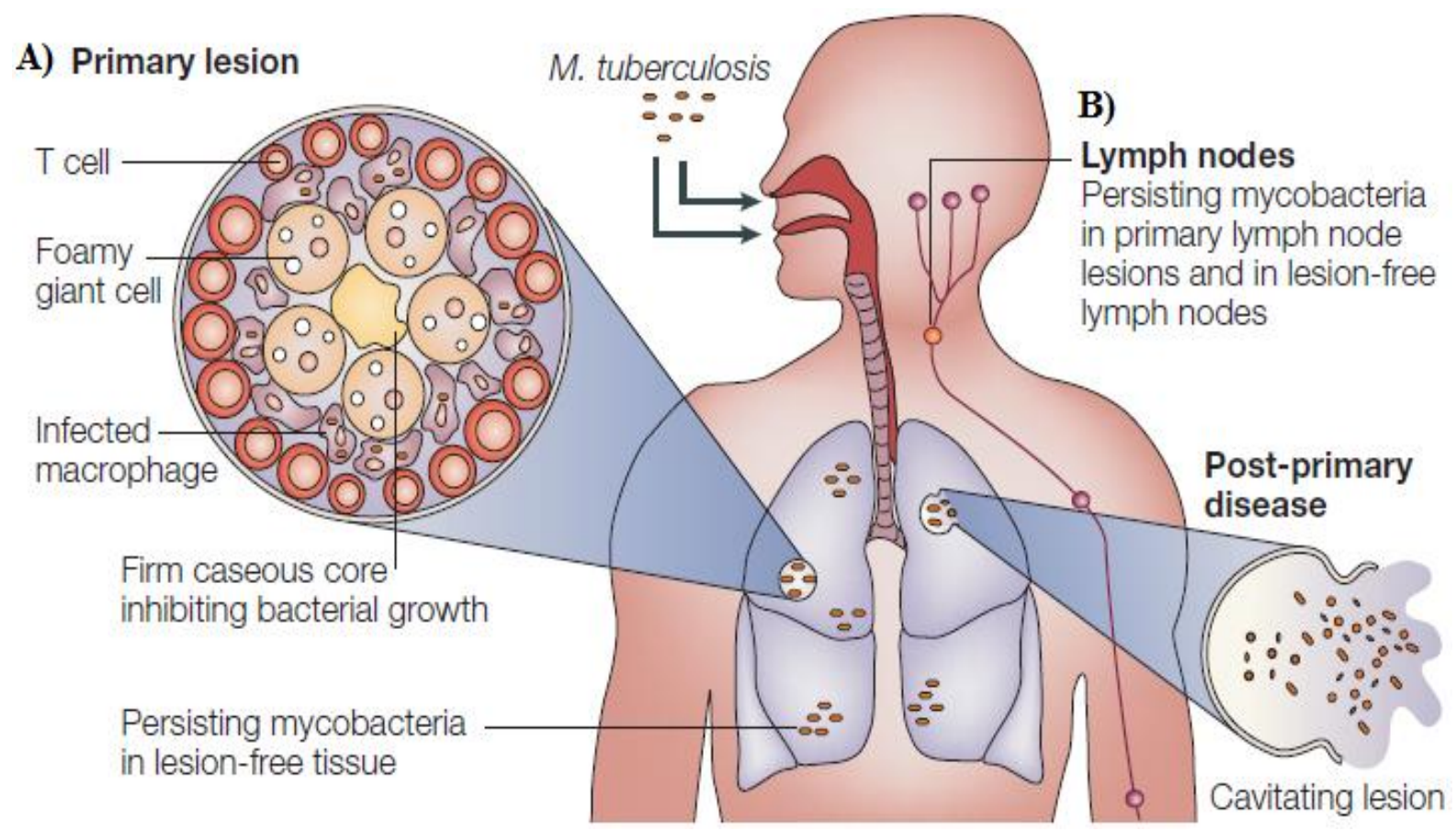

Figure 1.6: Pathology of Tb. After inhaling M. tuberculosis-containing tiny droplets from environment, initial M. tuberculosis infection is established in primary lesions. (A) Depicts the morphology of the primary lesion and (B) Continuous infection spreads through the lung tissues and lymph nodes to cause an active disease by involving the apical area of the lungs (see Figure 1.1). M. tuberculosis has also been known to cause inflammatory pseudotumors (lesions) in the lungs among immunocompromised patients [132-133], including transplant patients and people suffering with HIV/AIDS [134-135]. Figure reproduced with permission from Stewart et al. [110]. 


\subsection{Tb in New Zealand}

The New Zealand Ministry of Health reported about 10 new cases of Tb per 100,000, along with a higher annual incidence rate among Pacific Islanders [136]. The number of new $\mathrm{Tb}$ cases was higher compared to cases reported by other industrialised countries like the United States and Australia [137]. However, the annual $\mathrm{Tb}$ incidence rate has been recently decreased to 7 cases per 100,000 in New Zealand [138]. According to the report published by the Institute of Environmental Science and Research Limited (ESR), there were a total of 661 incident Tb cases in 2010 in New Zealand [139]. Generally the annual Tb incidence rates are 5 times higher in Maori people compared to other New Zealanders [140], and is the rates are 10 times higher in Pacific people compared to Europeans [138]. New Zealand has been included on the WHO list of countries where at least 0-3 cases of multiple drug-resistant $\mathrm{Tb}$ were reported during the period of 1994-2009 (Figure 1.7). Since, among immigrants, disease rates are higher in patients from Asian ethnic background [138], this could be due to a high influx of Asian immigrants to New Zealand and lack of proper case diagnosis [141]. Furthermore, a consistent increase in drug resistance is a concern for the NZ health industry (section 1.8.3.5.1). Apart from a rise in the annual number of MDR cases, Goh et al have recently reported the first ever XDR-Tb case in New Zealand history [142].

Figure 1.7 highlights the distribution of MDR-Tb in new $\mathrm{Tb}$ cases from New Zealand and other countries worldwide. 


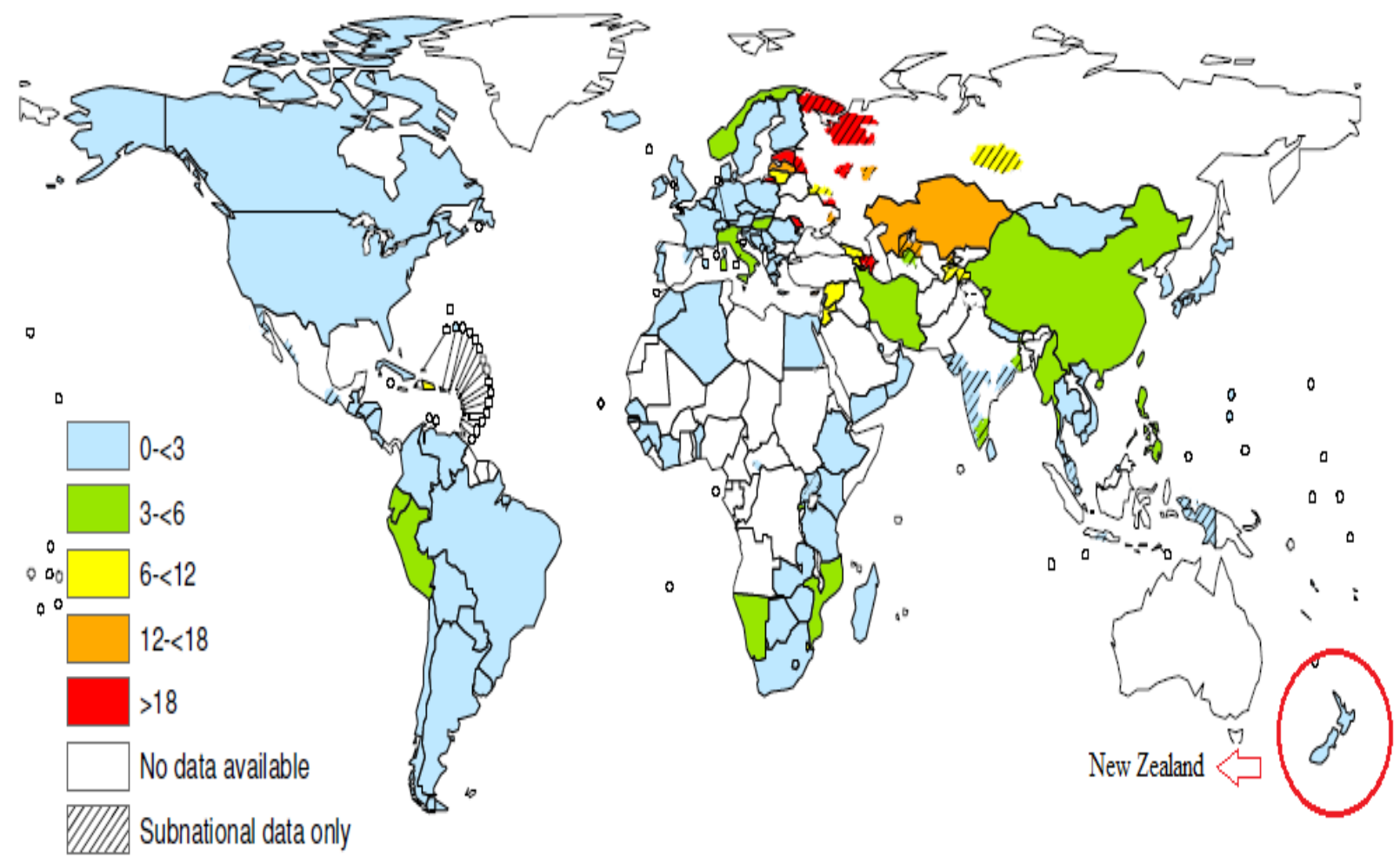

Figure 1.7: Worldwide distribution of MDR-Tb among new Tb cases. A blue box on top left shows up to three MDR-Tb cases reported annually within New Zealand during the period of 1994 to 2010. Figure was adapted with permission from Anti-Tb Drug Resistance Surveillance, World Health Organisation, [143]. 


\subsection{Tb Treatment, Therapeutics and Control Measures}

\subsubsection{Case Diagnosis}

Diagnostic laboratories have a major role in controlling $\mathrm{Tb}$ [144-145]. The tuberculin skin test is an old, yet currently used method to detect M. tuberculosis infections [28]. A delayedtype hypersensitive reaction is produced in response to specific $M$. tuberculosis antigens (tuberculin) such as PPD (Purified Protein Derivative). Attenuated PPD is given intradermally to check the host immune response against $M$. tuberculosis-specific antigen after 48-72 hours and is highly sensitive for LTBI detection [146]. An induration of $\geq 5 \mathrm{~mm}$ (for people who have had a close contact with $\mathrm{Tb}$ patients) or $\geq 15 \mathrm{~mm}$ (for low-risk $\mathrm{Tb}$ patients) indicates a positive tuberculin reaction [28]. While previous BCG vaccination does not have any effect over tuberculin reaction [147], in some cases, the sensitivity and reliability of tuberculin test is low [148]. Highly Tb specific antigens ESAT-6 (EarlySecreted Antigenic Target) and CFP-10 (Culture Filtrate Protein) are recommended for the detection of $M$. tuberculosis infection among BCG vaccinated individuals [149].

Nucleic acid amplification has long been considered as effective diagnostic tool for the detection of mycobacterial infections [150-154], particularly real-time PCR (Polymerase chain reaction) [155]. Recent advances in the field of molecular biology have provided highly sensitive and efficient testing e.g. $\mathrm{T}$-spot $\mathrm{Tb}$ test and interferon-gamma-release assays (more specific than conventional tests), performed on blood to detect latent infections more accurately [156-157], and these could be the methods of choice to diagnose Tb infections in the future [158]. For active Tb cases, smear testing has been considered as rapid, convenient and cost-effective tool to detect AFBs (Figure 1.3) in sputum samples [159-160]. Sensitivity of acid-fast smears can be improved by increasing volume of sputum samples [161]. A chest radiograph revealing lesions further helps to identify the active state of the disease (Figure $1.1)$. 


\subsubsection{Tb Treatment}

WHO has put intensive efforts by implementing a stringent DOTS strategy (Directly Observed Therapy Short-course) to control the growing burden of Tb after it was declared a health emergency in 1993 [162]. For drug-sensitive $\mathrm{Tb}$, a range of synergistic antibiotics are prescribed (Table 1.2) for an optimal therapy to clear the infection and to avoid relapses [163]. Both active disease and LTBI are treatable; however, patient's adherence to prescribed medicines is necessary in order to achieve complete cure. Treating LTBI can significantly reduce the subsequent disease burden [164]. First and second line anti-tubercular drugs, with different efficacies, are used to treat $M$. tuberculosis infections. Bacterial culture and susceptibility testing are usually required before and during the treatment to detect the sensitivity pattern of $M$. tuberculosis in order to appropriately alter the course and dose of antibiotics [165]. Standard frontline and reserve anti-Tb drugs are given in appropriate combinations depending on type and stage of infection (section 1.8.2.2.1).

\subsubsection{Standard Tb Drugs}

Current $\mathrm{Tb}$ treatment regimen is comprised of rifampicin (RIF), isoniazid (INH), ethambutol (ETH), streptomycin (STP) and pyrazinamide (PYZ) [166]. Most of first line anti-Tb drugs (Table 1.2, Figure 1.8) possess bactericidal activity and are highly active against $M$. tuberculosis. Second line drugs are not the preferred choice due to low safety but are in use in combination with first line drugs, particularly for the treatment of drug resistant $\mathrm{Tb}$ [167]. Reserve groups of these drugs derive from various antibiotic classes e.g. fluoroquinolones, aminoglycosides, cycloserine, thioamides, $p$-amino salicylic acid and polypeptides. For chemical structures of first and second line drugs, see figures 1.8 and 7.10, respectively. Specific mode of action of these drugs is presented in table 1.3. 
Table 1.2: Standard anti-Tb drugs and year of discovery

\begin{tabular}{lccl}
\hline Drugs & Discovery year & Target & Activity \\
\hline First-line available drugs & & & \\
Rifampicin & 1963 & RNA polymerase & Bactericidal \\
Isoniazid & 1952 & Cell wall & Bactericidal \\
Pyrazinamide & 1952 & Ribosome & Bactericidal \\
Ethambutol & 1962 & Cell wall & Bacteriostatic \\
Second-line available drugs & & & \\
Streptomycin & 1944 & Ribosome & Bactericidal \\
Ciprofloxacin & 1987 & DNA gyrase & Bactericidal \\
Capreomycin & 1960 & Ribosome & Bacteriostatic \\
Ethionamide & 1956 & Cell wall & Bacteriostatic \\
Aminosalicylic acid & 1949 & thyA gene & Bacteriostatic \\
Amikacin & 1970 & rRNA & Bactericidal \\
D-Cycloserine & 1955 & Cell wall & Bacteriostatic \\
\hline
\end{tabular}

Reviewed in Ying Zhang 2005 [168]

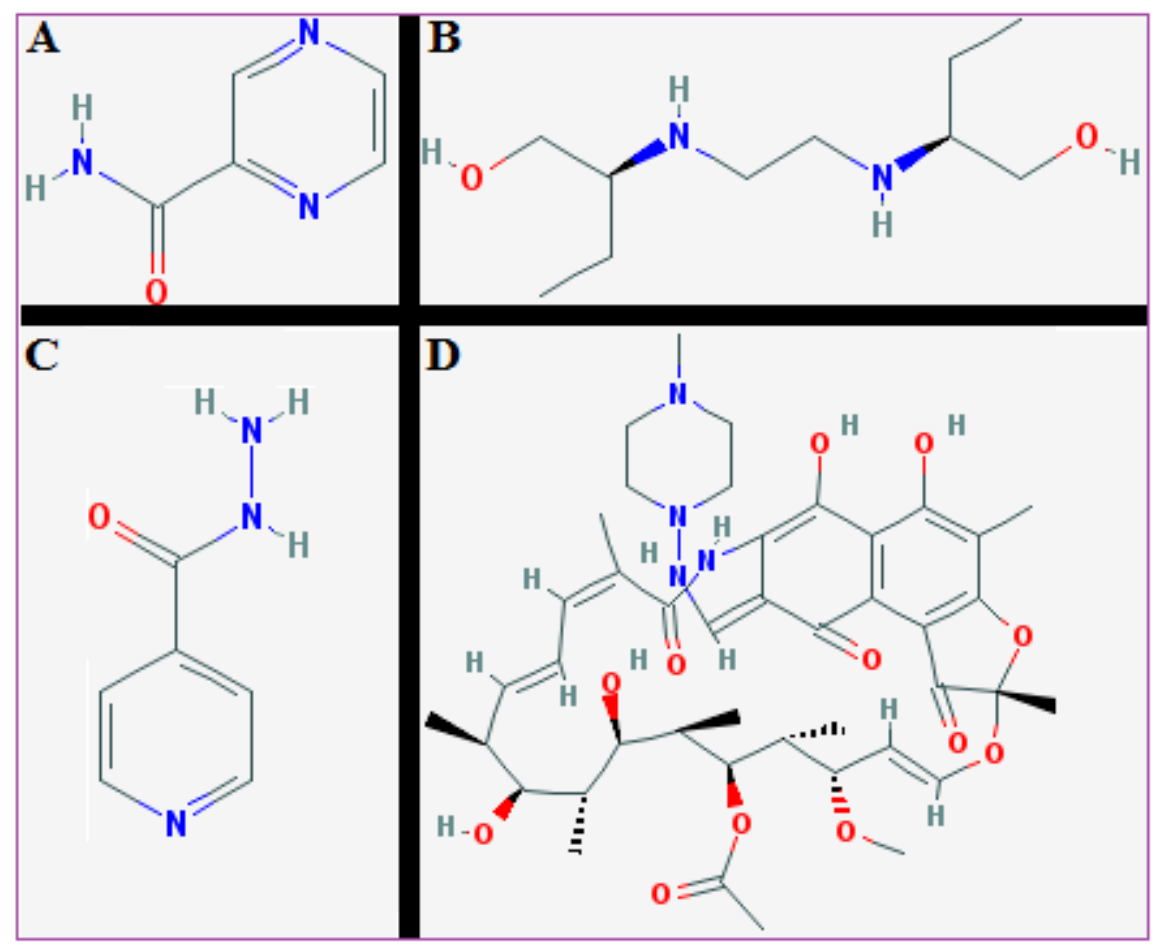

Figure 1.8: Current panel of Tb drugs comprising front-line defence (A) Pyrazinamide, $M F \mathrm{C}_{5} \mathrm{H}_{5} \mathrm{~N}_{3} \mathrm{O}$ and MW $123.11 \mathrm{~g} / \mathrm{mol}$ (B) Ethambutol, MF $\mathrm{C}_{10} \mathrm{H}_{24} \mathrm{~N}_{2} \mathrm{O}_{2}$ and MW $204.30 \mathrm{~g} / \mathrm{mol}$ (C) Isoniazid, MF $\mathrm{C}_{6} \mathrm{H}_{7} \mathrm{~N}_{3} \mathrm{O}$ and 
MW $137.13 \mathrm{~g} / \mathrm{mol}$ and (D) Rifampicin, MF $\mathrm{C}_{43} \mathrm{H}_{58} \mathrm{~N}_{4} \mathrm{O}_{12}$ and MW $822.94 \mathrm{~g} / \mathrm{mol}$ (chemical structures of drugs were retrieved from the PubChem database). See figure 7.10 for chemical structure of second-line drugs and table 1.3 for their mode of action. MF, molecular formula. MW, molecular weight.

Table 1.3: Standard anti-Tb drugs and mechanisms of action

\begin{tabular}{|c|c|}
\hline Drugs & Mode of Action \\
\hline
\end{tabular}

Rifampicin (RIF) RIF blocks transcription by binding with the $\beta$-subunit of DNAdependant RNA polymerase

Isoniazid (INH) INH is a pro-drug which gets activated by the $k a t \mathrm{G}$ gene product and inhibits mycolic acid synthesis

Pyrazinamide (PYZ) PYZ inhibits trans-translation

Ethambutol (ETH) ETH inhibits the enzyme, arabinosyl transferases, involved in cell wall biosynthesis

Streptomycin (STP) STP inhibits protein synthesis by binding with the 30 S ribosomal subunit

Ciprofloxacin (CIP) Halts DNA replication by inhibiting the DNA gyrase enzyme

Capreomycin (CAP) Inhibits protein synthesis by interfering with ribosomal assembly [174]

Ethionamide (ETM) Inhibits enoyl-ACP reductase coded for by the inhA gene involved in mycolic acid synthesis for cell wall construction

D-Cycloserine (CYS) Inhibits alanine racemase and blocks the synthesis of peptidoglycan

Reviewed in the Handbook of Tuberculosis Drugs 2008 [178] and Gillespie. SH 2002 [179] 


\subsubsection{Tb Control Measures}

BCG is the only vaccine (section 1.3.2.1) available to protect children from $M$. tuberculosis infection with nearly no efficacy in patients who already have Tb. Therefore, new and improved vaccines are required to tackle the resurgence $\mathrm{Tb}[180]$.

\subsection{DOTS Therapy}

After the WHO had launched DOTS program in 1994 for more integrated infection control, drug-susceptible $\mathrm{Tb}$ is treated as per standard recommendations to ensure patients adherence and treatment success [166]. DOTS proved to be effective for disease control and has been successful in all countries and territories with a significant impact on reducing the $\mathrm{Tb}$ incidence [181]. For latent infections, a nine-month isoniazid monotherapy followed by rifampicin and pyrazinamide (daily for two months), is recommended with DOTS [28, 166, 182-183]. However, regimen may be variable in medication doses, toxicities and monitoring requirements depending on individual patient's situation [28]. While nine-month isoniazid therapy is preferred, six-month isoniazid regimen may provide an equal protection in both non-HIV infected and HIV-infected patients [184-185]. For active Tb, treatment is given in two phases; initial phase and eradication phase, with a combination of first and second-line drugs. Initial intensive phase includes chemotherapy with rifampicin and isoniazid along with pyrazinamide and ethambutol for the first two months, followed by rifampicin and isoniazid for at least next four months in the continuation phase to complete the treatment course (Table 7.1).

Anti-tubercular therapy associated toxicity such as skin rash, fever, peripheral neuropathy and liver toxicity, has been reported previously and may require discontinuation of the treatment [186-188]. Adverse effects generally appear in the early stages of the treatment therefore, evaluation of patient's tolerance towards anti-Tb drugs is important for the 
successful treatment outcomes. Several risk factors for isoniazid-associated hepatotoxicity such as age (over 35 years), female gender and increase use of alcohol, have been documented [189-192]. Specific guideline for use of isoniazid in the treatment of LTBI are available including assessment of risk factors and clinical monitoring of hepatotoxicity [193].

\subsubsection{Drug Resistance}

Despite long and exhaustible Tb treatment, failure of DOTS strategy is not the only reason for the development of resistance to available drugs. An additional factor is lack of monitoring current drug resistance levels in communities with a high HIV burden [194]. Therefore, fast emerging drug resistant $\mathrm{Tb}$ has to be effectively controlled in order to successfully implement the mutual TB-HIV control programs [195]. M. tuberculosis has a resilient nature as it renders itself resistant towards the available drugs over time (section 1.8.3.1), and this makes it hard and expensive to treat. Resistance can vary in type depending on the strains and severity of the disease e.g. multiple drug resistance (MDR), extensive-drug resistance (XDR) and extremely-drug resistant (XXDR) forms of Tb [196].

Extremely drug-resistant $\mathrm{Tb}$ indicates an endpoint for resistance where $M$. tuberculosis becomes resistant to all first-line and second-line anti-Tb drugs [197]. A number of factors are involved in developing drug resistance such as patient's non compliance towards treatment including self-medication, non standard regimens (lack of proper treatment), inadequate drug supply, status of the patient's immune system, antibiotic selective pressure and any pre-existing resistance present in the infecting strain [198]. While such factors can generate multi-drug resistant strains, there is a chance for dissemination of these resistant strains in the community. Other contributing factors include lack of awareness, poverty and socioeconomic issues. These risk factors need to be observed regularly to evaluate the impact of interventions [199]. 


\subsubsection{Resistance Due to Genetic Mutations}

While intrinsic resistance in mycobacterial species to some drugs is generally attributed to their thick and less permeable cell wall, genetic basis of drug resistance have been identified in mycobacteria [200]. Specific mutations in genes could lead to altered drug susceptibility patterns [201]. Role of mutations and associated drug resistance in M. tuberculosis has previously been demonstrated $[116,202]$ (section 4.3.1.1). A patient with active Tb could possibly be infected with more than one strain at a time, and therefore, genetic mutations could hamper successful treatment [203]. Gaining an insight into molecular mechanisms underlying drug resistance in $M$. tuberculosis (with availability of complete genome sequence [17]) would be helpful for the development of new anti-Tb drugs.

Table 1.4: Genetic basis of drug resistance in M. tuberculosis

Anti-Tb Drug Mechanism of action Genes involved Reren

Isoniazid Inhibition of mycolic acid biosynthesis $k a t \mathrm{G}$, inh $\mathrm{A}$ and $\operatorname{ahpC} \quad$ Vilchèze et al. ${ }^{[170]}$

Rifampicin $\quad$ Inhibition of transcription $\quad r p o \mathrm{~B} \quad$ Wehrli et al. ${ }^{\text {[204] }}$

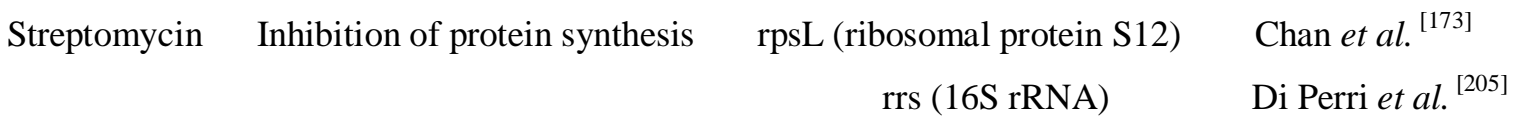

\begin{tabular}{|c|c|c|c|}
\hline \multirow[t]{3}{*}{ Ethambutol } & Inhibition of arabinogalactan & $e m b \mathrm{~A} / \mathrm{B}$ & Ramaswamy et al. ${ }^{[206]}$ \\
\hline & \& lipoarabinomannan biosynthesis & (arabinosyl transferase) & Belanger et al. ${ }^{[207]}$ \\
\hline & & $e m b \mathrm{C}$ & Goude et al. ${ }^{[208]}$ \\
\hline \multirow[t]{2}{*}{ Pyrazinamide } & inhibits trans-translation ${ }^{[171]}$ & pncA & Scorpio et al. ${ }^{[209]}$ \\
\hline & $(\mathrm{pyr}$ & zinamidase-nicotinamidase) & Davies et al. ${ }^{[210]}$ \\
\hline
\end{tabular}

Fluoroquinolones Inhibition of DNA gyrase $\quad$ gyrA (DNA gyrase subunit A) Telenti A. ${ }^{\text {[202] }}$ 


\subsubsection{Phenotypic Drug Resistance}

The term 'phenotypic drug resistance' refers to $M$. tuberculosis cells that are not metabolically active and replicate at an extremely slow growth rate; therefore, these cells develop temporary resistance towards drugs. Persister cells have been found to promote drug tolerance that leads towards multiple drug resistance to the available chemotherapy [211].

\subsubsection{Multiple Drug Resistant Tb (MDR-Tb)}

Multi-drug resistance (MDR) is defined as a state in which M. tuberculosis is resistant to the two major front line anti-Tb drugs, rifampicin and isoniazid [205]. MDR-Tb outbreaks have been reported by many countries worldwide [212-214], and it has been considered a serious concern for the global $\mathrm{Tb}$ drug resistance surveillance programs [215]. The WHO has estimated 0.65 million prevalent MDR-Tb cases from the pool of 12 million globally prevalent $\mathrm{Tb}$ cases in 2010 [100]. According to WHO report 2011, the annual number of MDR cases is increasing, with at least 27 high burden MDR-Tb countries reported so far [100]. Adequate control of MDR-Tb is required as these growing number of MDR cases is a worrying factor [216-217], that demands for the formulation of an effective regimen [218].

\subsubsection{Extensively Drug Resistant Tb (XDR-Tb)}

$\mathrm{XDR}-\mathrm{Tb}$ is a more severe form of MDR-Tb, in which $M$. tuberculosis is resistant to at least two front line anti-Tb drugs, rifampicin and isoniazid along with resistance to fluoroquinolone and at least one of the three injectable aminoglycosides. XDR-Tb been considered a threat to human health [219], and also for the effectiveness of global $\mathrm{Tb}$ control programs [220]. It is virtually impossible to exterminate XDR-Tb along with complications such as age factor, treatment failure and death [221]. The possibility of re-infection with a 
drug-resistant strain is not common during chemotherapy; hence, it is not usually the cause of treatment failure, indicating that the potential problem of drug resistance is a more serious concern [222].

\subsubsection{XDR-Tb at the Global Level}

To date 58 countries have been reported by the WHO to have extensively drug resistant strains of M. tuberculosis [223], with Asia and Africa being the major contributors [224-225]. Growing numbers of XDR-Tb cases is a worrying factor and concern for meeting the target of reversing the incidence of Tb by 2015 compared to 1990 through the Stop Tb Partnership and Millennium Development Goals [226].

\subsection{XDR-Tb in New Zealand}

Fortunately, the situation for drug-resistant $\mathrm{Tb}$ is not threatening in New Zealand. However, New Zealand Institute of Environmental and Scientific Research has calculated the annual MDR-Tb incidence to be $0.8 \%$ with an XDR-Tb case reported in 2010 [142]. If not controlled, XDR-Tb could be a major challenge to disease eradication [227], and may also present potential concern for the Australasian health industry [228-229].

\subsubsection{Extremely Drug Resistant Tb}

A recently reported new form of $\mathrm{Tb}$ defined as super extensively drug-resistant $\mathrm{Tb}$ (XXDR$\mathrm{Tb}$ ), exists in which $M$. tuberculosis does not respond to any anti-Tb drugs and is therefore impossible to treat [196], [230]. If the numbers of XXDR-Tb cases keep on rising, it will not only jeopardise the Stop-Tb plan worldwide but also will create further complications for global $\mathrm{Tb}$ control and management agencies. 


\subsubsection{Tb and HIV Co-infection}

Human Immunodeficiency Virus (HIV) infection serves as a driving force for the high prevalence of $\mathrm{Tb}$, due to host immunodeficiency [231]. Therefore, the HIV pandemic has had a huge impact on the global Tb burden (section 5.1.1). Despite the availability of an antiinfective chemotherapy, such dual infections continue to escalate relentlessly, especially in the developing world as the WHO has estimated 2.1 million $\mathrm{HIV}$-associated $\mathrm{Tb}$ cases and 0.35 million deaths in 2010 worldwide [100]. Furthermore, due to the synergistic effect of $\mathrm{HIV}$ on $\mathrm{Tb}$ infection, the prevalence and mortality rates for $\mathrm{Tb}$ are increasing. Unfortunately, currently available anti-Tb drugs are not compatible with most retroviral drugs used to treat HIV infection. Therefore, the situation requires modification to the current regimen by introducing new drugs with high compatibility for the treatment of dual infections (Chapter $5)$.

Nosocomial transmission of $\mathrm{Tb}$ infection to laboratory staff and official health care workers has been previously reported among immuno-compromised people [232]. People with low immunity occasionally can catch hospital-acquired infections caused by non-tuberculous mycobacteria as well, along with multiple infections as a result of their immune deficiency [233]. Moreover, smoking and alcohol have been considered as contributing factors to the disease [234-235]. There is an urgent need to produce new and more effective drugs to control and cure Tb. Sir John Crofton reviewed some aspects of past Tb research highlighting the lack of novel chemotherapies that has forced us to rely on old anti-Tb drugs for decades.

"It is a sad reflection on society's incompetence that, more than thirty years after the methods for cure and prevention were evolved and before the advent of the HIV epidemic, there were already more patients with active tuberculosis in the world than there had been in the 1950s" 
$\mathrm{Tb}$ research has undoubtedly made progress in terms of reducing the global $\mathrm{Tb}$ burden over the last decade [100], yet there are challenges that need to be overcome [237]. The current drug regimen is over three decades old and is complemented with a huge daily pill burden, requiring 6-12 months (depending on the type of infection) to treat drug-sensitive $\mathrm{Tb}$ (Table 7.1). Novel anti-Tb drugs for use in combination therapy and with different mode of action are clearly required that are also synergistically active with anti-retroviral therapy against HIV infections. New drugs are also expected to reduce the treatment time and to overcome the growing burden of drug-resistant $\mathrm{Tb}$ [238].

\subsubsection{Current Pipeline for New Anti-Tb Drugs}

While many candidates are currently in the clinical pipeline for $\mathrm{Tb}$ drug development, no new anti-Tb drugs have been marketed for over three decades. Only few of them have reached phase three such as moxifloxacin and gatifloxacin (Figure 5.20).

\subsubsection{Research Hypothesis}

Two important hypotheses were to be tested in this study;

1. M. smegmatis is limited in its ability to detect inhibitors that are active against $M$. tuberculosis.

2. Mycobacterial inhibitors with new modes of action can be found from non-targeted screening of chemical libraries.

The main objectives of this research are given below (section 1.8.6.1). 


\subsubsection{Aims of This Research}

1. To develop a sensitive assay for determining inhibitory activity in the slow-growing Tb strains, M. bovis BCG and M. tuberculosis H37Ra.

2. To compare various in vitro $\mathrm{Tb}$ models previously used for anti- $\mathrm{Tb}$ drug screening to demonstrate their better ability and sensitivity to specifically identify $M$. tuberculosis inhibitors.

3. To validate newly-identified inhibitors against virulent $M$. tuberculosis in vitro which have the potential to be developed into therapeutic drugs. Also to test these inhibitors further with respect to their bactericidal activities against $M$. tuberculosis and their efficacy against other human pathogens.

4. To test the hypothesis that development of a dual inhibitor of Tb/HIV is possible.

There are several methods available for screening mycobacterial inhibitors e.g. in vitro models, intra-macrophage infection models and various in vivo models [239]. Due to the pathogenic nature of $M$. tuberculosis $\mathrm{H} 37 \mathrm{Rv}$, an avirulent $\mathrm{Tb}$ strain, M. bovis BCG, was employed as a drug-screening model in this study using a physical containment level 2 laboratory that was available for high-throughput screens (HTS) at Victoria University of Wellington. 
Chapter 2

Materials and Methods 


\subsection{Bacterial Strains and Plasmids}

\subsubsection{Bacterial Strains}

Various bacterial (and mycobacterial) strains were used in this study and are listed in table

2.1 along with their types and relevant details. All bacterial strains were made up in $20 \%$ final concentration of glycerol and were stored at $-80{ }^{\circ} \mathrm{C}$.

Table 2.1: Bacterial strains used in this study

\begin{tabular}{|c|c|c|c|}
\hline Strain & Type $^{\mathrm{a}}$ & Virulence & Source or Reference \\
\hline E. coli $\mathrm{DH} 5 \alpha$ & Plasmid storage strain & non-pathogenic & Hanahan D 1985 [240] \\
\hline E. coli lab strain & ATCC25922 & non-pathogenic & ESR Labs $^{1}$ \\
\hline E. coli lab strain & BL21 (DE3) & non-pathogenic & Novagen \\
\hline Staphylococcus aureus & ATCC25923 & non-pathogenic & ESR Labs ${ }^{1}$ \\
\hline Staphylococcus aureus & Newman $g f p$ labelled & non-pathogenic & Earl et al. [241] \\
\hline Pseudomonas aeruginosa & ATCC25668 & non-pathogenic & ESR Labs $^{1}$ \\
\hline \multicolumn{2}{|c|}{ Mycobacterium smegmatis mc $^{2} 155$ ATCC607 } & avirulent strain & Snapper et al. 1990 [242] \\
\hline Mycobacterium bovis $\mathrm{BC}$ & Pasteur strain $1173 \mathrm{P} 2$ & non-pathogenic & $\mathrm{MIMR}^{2}$ \\
\hline M. bovis BCG-GFP & Pasteur strain $1173 \mathrm{P} 2$ & non-pathogenic & Luo et al. 1996 [243] \\
\hline \multicolumn{2}{|c|}{ Mycobacterium tuberculosis H37Ra ATCC25177 } & avirulent strain & Wang et al. 2007 [244] \\
\hline \multicolumn{2}{|c|}{ Mycobacterium tuberculosis H37Rv ATCC27294 } & virulent $^{3}$ & Steenken et al. 1934 [245] \\
\hline
\end{tabular}

\footnotetext{
${ }^{a}$ American Type Culture Collection. ${ }^{1}$ Laboratory strains were imported from New Zealand reference laboratory, Institute of Environmental and Scientific Research (ESR), Porirua, Wellington. ${ }^{2}$ Malaghan Institute of Medical Research, Wellington, New Zealand. ${ }^{3}$ PC3 facility was used at Johns Hopkins Centre for Tuberculosis Research, USA.
} 


\subsubsection{Plasmids Used in This Study}

Several types of plasmids were used in this research including new constructs. Two new constructs, pET28a+_Rv2228c and pET28a+_Rv2902c were generated in this study and are listed in table 2.2. Plasmid speciality, relevant characteristics and their source are given in table 2.2 whereas, plasmid maps are presented in the appendix section 7.3 (Miscellaneous figures). 
Table 2.2: Bacterial plasmids used in this study

\begin{tabular}{|c|c|c|}
\hline Plasmid & Description & \\
\hline pHS201 & $\begin{array}{l}\text { Mycobacterial-E. coli shuttle vector with a high copy number } \\
\text { version of the pAL5000 mycobacterial replicon, BCG } h s p 60 \\
\text { promoter, } g f p \text { with a synthetic Shine-Delgarno sequence } \\
\operatorname{kan}^{\mathrm{r}} \text {. Size } 5.813 \mathrm{~kb}\end{array}$ & [246] \\
\hline pSHIGH_hsp60 & $\begin{array}{l}\text { A high copy number } E \text {. coli shuttle vector for mycobacteria. } \\
\text { OriM, BCG } h s p 60 \text { promoter, gfpmut } 2 \text { and } \operatorname{kan}^{\mathrm{r}} \text {. Size } 5.6 \mathrm{~kb}\end{array}$ & [247] \\
\hline pYL_gfp & $\begin{array}{l}\text { BCG-E. coli shuttle vector. Two constitutively } \\
\text { expressed BCG promoters } h s p 60 \text { and } h s p 70, \operatorname{kan}^{\mathrm{r}}\end{array}$ & {$[243]$} \\
\hline pET28a (+) & $\begin{array}{l}\text { Protein over-expression vector, pBR322 origin, } \\
\text { T7 promoter, T7 transcriptional start and terminator, } \\
\text { His•Tag }{ }^{\circledR}, \mathrm{MCS}, \mathrm{Kan}^{\mathrm{r}}-\text { size } 5.369 \mathrm{~kb}\end{array}$ & Novagen \\
\hline $\mathrm{pCR}^{\circledR} 2.1$ & $\begin{array}{l}\text { TA cloning vector for direct ligation of PCR products, } \\
\text { pUC origin, T7 promoter/primer site, LacZ } \alpha \text { fragment, } \\
\text { M13F and M13R priming site, Amp }{ }^{\mathrm{r}}, \mathrm{kan}^{\mathrm{r}} \text {. Size } 3.9 \mathrm{~kb}\end{array}$ & Invitrogen \\
\hline pCG79 & $\begin{array}{l}\text { Mycobacterial- } E \text {. coli shuttle vector containing the } \\
\text { pAL5000 and pUC18 mycobacterial replicons. Also carries } \\
\text { Tn611 sequence and cassette for } \mathrm{kan}^{\mathrm{r}} \text { - size } 17.9 \mathrm{~kb}\end{array}$ & [248] \\
\hline pET28a (+)_Rv2 & $\begin{array}{l}\text { 2228c Derivative of pET28a (+) with a } 1.095 \mathrm{~kb} \text { PCR fragment } \\
\text { Rv2228c, encodes RNase HI from } M \text {. tuberculosis H37Rv } \\
\text { cloned in EcoRI and XhoI, total size of plasmid } 6.468 \mathrm{~kb}\end{array}$ & This study \\
\hline pET28a (+)_Rv2 & $\begin{array}{l}\text { 902c Derivative of pET28a (+) with a } 0.795 \mathrm{~kb} \text { PCR fragment } \\
\text { Rv2902c, encodes RNase HII from M. tuberculosis H37Rv } \\
\text { cloned in EcoRI and XhoI, total size of plasmid } 6.167 \mathrm{~kb}\end{array}$ & This study \\
\hline
\end{tabular}


Table 2.3: Mammalian cell lines used in this study

Cell line

Description

HL-60 Non-adherent human promyelocytic leukaemic cell line

Vero $\quad$ Non-adherent human cell line

J774.A1 Adherent murine macrophage cell line

\subsection{Growth Media}

\subsubsection{Growth Requirements for the Slow-growing Mycobacterial Strains}

All M. tuberculosis and M. bovis BCG cultures were grown at $37{ }^{\circ} \mathrm{C}$ in Middlebrook $7 \mathrm{H} 9$ (Becton Dickinson Ltd) broth and on Middlebrook 7H10 agar plates for up to two and four weeks, respectively.

\subsubsection{Media Supplements}

- OADC

OADC stands for Oleic acid, albumin, dextrose and catalase. It is a special nutrient supplement for the cultivation $M$. tuberculosis complex. Oleic acid provides long-chain fatty acids for metabolism, albumin binds any toxins to protect $M$. tuberculosis, dextrose is a energy source in the form of carbon and catalase enzyme catalyzes iron with molecular oxygen to stimulate revival of any damaged bacilli. Filter-sterilized OADC has to be added into $7 \mathrm{H} 9$ broth at about $50-55{ }^{\circ} \mathrm{C}$ to avoid denaturation of additional nutrients and essential proteins. 


\section{OADC Preparation (for 1L)}

1. Combine $925 \mathrm{~mL}$ deionised water and 8.5 grams $\mathrm{NaCl}$ in a 2 litre flask. Stir with a magnetic stir bar until salt is dissolved.

2. Add 50 grams bovine serum albumin Fraction $\mathrm{V}$ and stir until completely dissolved.

3. Add 20 grams dextrose and stir until completely dissolved.

4. Add $50 \mathrm{~mL}(1 \%)$ sodium oleate solution and stir until completely mixed. The $\mathrm{pH}$ should be around 7.0.

5. Filter sterilize through $0.22 \mu \mathrm{m}$ Millipore syringe filter and store at $4{ }^{\circ} \mathrm{C}$.

\section{A-Sodium Oleate Solution}

Sodium oleate is an additive for the OADC supplement for $M$. tuberculosis growth media.

Ingredients (for $500 \mathrm{~mL}$ )

\section{$12.5 \mathrm{~mL} 2 \mathrm{M} \mathrm{NaOH}$}

$6.0 \mathrm{~mL}$ oleic acid (specific gravity for oleic acid, $1 \mathrm{~g} / 1.2 \mathrm{~mL}$ )

$481.5 \mathrm{~mL}$ water

\section{B- Protocol}

Combine the above ingredients in a $(1 \mathrm{~L})$ flask. Stir with a magnetic stir bar until completely dissolved. Filter sterilize through a $0.22 \mu \mathrm{m}$ millipore filter. Store at room temperature wrapped in aluminium foil as oleic acid is light sensitive.

\section{- $\quad$ Tween80}

Tween80 is a detergent that is used as surfactant for mycobacterial cultures. Mycobacteria tend to make clumps during in vitro growth. Therefore, apart from mild agitation, Tween80 is 
used to disrupt the clumps in culture and for smoother mycobacterial growth kinetics [249]. Stock solutions, $10 \%(\mathrm{w} / \mathrm{v})$ were prepared in distilled and deionised water $\left(\mathrm{ddH}_{2} \mathrm{O}\right)$ and stored at $4{ }^{\circ} \mathrm{C}$. Sterilization was achieved using an autoclave (Siltex 250D Global Science) at $121{ }^{\circ} \mathrm{C}$ for 30 minutes. Unless otherwise stated, the final concentration of Tween80 was $0.05 \%$ (v/v) for all M. bovis BCG and M. tuberculosis cultures.

\section{- Glycerol}

Glycerol was used as an additional carbon source to yield better M. tuberculosis growth at final concentrations of $0.05 \%$. Glycerol stocks of $80 \%, 50 \%$ and $10 \%$ were prepared with distilled water and stored at $4{ }^{\circ} \mathrm{C}$ after autoclaving in a standard laboratory autoclave used for complete sterilization.

\section{- Antibiotics}

Unless otherwise stated, all antibiotics were purchased from Sigma Aldrich Company and were resuspended in DMSO or water depending on their respective solubilities. All drugs and inhibitors (freezer stocks and working stocks) were stored at $-20{ }^{\circ} \mathrm{C}$.

\subsubsection{Enrichment Media for the Slow Growers}

All M. tuberculosis-specific media were supplemented for further enrichment with:

- $10 \%$ OADC (w/v) (Oleic acid, Albumin, Dextrose and Catalase)

- $\quad 0.05 \%$ Tween80 (v/v) and

- $0.5 \%$ Glycerol (v/v)

Sodium oleate solution is light sensitive and therefore the Schott bottle was wrapped with aluminium foil to protect it from light. 


\subsubsection{Starvation Media for the Slow Growers}

In contrast to the enriched culture condition, a starvation recipe (to achieve effective carbon starvation) was used to investigate the metabolic activity of the slow growers by formulating appropriate starvation conditions for in vitro culture. Objective of this exercise was to evaluate the response to various inhibitors of non-growing or extremely slow-growing $M$. tuberculosis cells that are still metabolically active. The aim was to use a medium that mimicked the hypoxic conditions of human granulomas. Effective carbon starvation was achieved when these medium conditions were used for M. bovis BCG (Figure 7.4, A-D). Cells were washed with PBS three times to reduce the nutrient carryover, and the mycobacteria were resuspended in sterile PBS before being seeded into assays.

- $7 \mathrm{H} 9$ broth

- $2.0 \%$ OADC (w/v) (Oleic acid, Albumin, Dextrose and Catalase)

- $\quad 0.025 \%$ Tween80 (v/v) and

- $0.1 \%, 0.05 \%$ and $0 \%$ glycerol $(\mathrm{v} / \mathrm{v})$

\subsubsection{Culture Conditions for the Slow Growers}

Standard growth conditions for both $M$. tuberculosis and M. bovis BCG cultures are as follows;

- Standard growth medium (sterile)

- Temperature $37^{\circ} \mathrm{C}$

- Shaking, 200 rpm (Shaking Incubator DK-S1020, Global Science)

All mycobacterial cultures (solid and liquid) were grown at $37^{\circ} \mathrm{C}$. Solid $7 \mathrm{H} 10$ plates were put in a non-shaking incubator for 3-4 weeks. Figure 7.3-II shows the growth of M. tuberculosis 
H37Ra on 7H10 plates supplemented with 10\% OADC. Liquid cultures either in $50 \mathrm{~mL}$ or 15 $\mathrm{mL}$ polypropylene tubes, $250 \mathrm{~mL}$ or $500 \mathrm{~mL}$ flasks or in 96-well plates (Ray Lab) were agitated in a shaking incubator with a shaking speed of $200 \mathrm{rpm}$. After placement of their lids, 96-well plates were sealed with a thin cellulose transparent seal (Inter lab) to avoid culture evaporation during longer incubations. Assay plates were also wrapped tightly with parafilm afterwards and placed in a marked polythene bag prior to incubation.

\subsubsection{Freezer Stocks}

For the long-term storage of mycobacterial strains, freezer stocks were prepared in duplicate aliquots for storage in a -80 freezer. An aliquot $(0.5 \mathrm{~mL})$ of a logarithmically growing culture of $M$. bovis BCG was transferred into $1.8 \mathrm{~mL}$ Cryotube $^{\mathrm{TM}}, 0.5 \mathrm{~mL} 80 \%$ glycerol was added and the cells were stored at $-80{ }^{\circ} \mathrm{C}$. Frozen cells were stable for several years.

\subsubsection{Mycobacterium smegmatis $\mathrm{mc}^{2} 155$}

M. smegmatis was grown in Luria-Bertani broth (LB) as well as in $7 \mathrm{H} 9$ broth with and without supplements (section 2.2.1.1). M. smegmatis was cultured with $50 \mu \mathrm{g} / \mathrm{mL}$ kanamycin (for plasmids pHS201 and pSHIGH_gfp). All M. smegmatis liquid cultures were placed in a shaking incubator with a shaking of $200 \mathrm{rpm}$ along with $0.1 \%(\mathrm{v} / \mathrm{v})$ final concentration of Tween80. For solid growth, $M$. smegmatis cultures were streaked on LB agar plates and were incubated in a non-shaking incubator at $37{ }^{\circ} \mathrm{C}$ for up to 96 hours.

\subsubsection{Escherichia coli, Pseudomonas aeruginosa and Staphylococcus aureus}

All strains were grown in Luria-Bertani broth (LB broth) along with relevant antibiotics, as required in the assay. To achieve solid agar growth, cultures were streaked on LB agar plates and incubated in a non-shaking incubator at $37{ }^{\circ} \mathrm{C}$ overnight (for all cultures). All liquid 
cultures were grown without adding Tween 80 and were incubated at $37{ }^{\circ} \mathrm{C}$ with (or without) shaking.

\subsection{Chemicals and Reagents}

Unless otherwise stated, all chemicals, reagent and drugs required for setting up growth inhibition assays were purchased from Sigma Aldrich. DNA markers, DNA loading buffer, dNTPs, IPTG, DNA ladder were purchased from Invitrogen, New Zealand. All individual novel chemical compounds identified in the screens as positive hits for validation experiments were imported from the National Institutes of Health (NIH), United States.

\subsection{Enzymes}

Unless otherwise stated, all restriction enzymes were purchased from New England Bio Labs (NEB, Auckland, NZ). RNase HI from E. coli and lysozyme were sourced from Sigma Aldrich. T4 DNA ligase and Bio Line's DNA Taq polymerase were purchased from Invitrogen and Qiagen, respectively.

\subsection{Oligonucleotides}

Oligonucleotide primers for DNA manipulation were designed using Gentle Software (version 2.1). The approximate primer length was about $30 \mathrm{bp}$ with an average GC content of about 45-55\%. Both forward and reverse complementation along with restriction site sequences were confirmed and verified before their synthesis by Integrated DNA Technologies (Belgium). All primers were resuspended in ddH2O (DNase and RNase free) to make $100 \mu \mathrm{M}$ stocks and $10 \mu \mathrm{M}$ working stocks were prepared for final use in downstream molecular biology applications. Primer sequences along with their respective genes from $M$. tuberculosis $\mathrm{H} 37 \mathrm{Rv}$ are given in table 2.4. 


\subsubsection{Primer Sequences for M. tuberculosis Genes Coding RNase HI/II Proteins}

Primers used to amplify and clone RNase HI/II enzymes from $M$. tuberculosis are depicted in table 2.4.

Table 2.4: Primers used in this work

\begin{tabular}{lccc} 
Primer Name & Gene & Product & Primer Sequence [5'-3'] \\
\hline Rv2228c_F & $r n h$ A & RNase HI & 5'-CTAG GAATTCGTGAAAGTTGTCATCGAAGC -3' \\
Rv2228c_R & - & - & 5'-CTAG $\underline{\text { CTCGAG CTATAGATAGCCTGTCTGATT-3' }}$ \\
Rv2902c_F & $r n h \mathrm{~B}$ & RNase HII & 5'-CTAG $\underline{\text { GAATTC ATGACCAAGACCTGGCCACCG-3' }}$ \\
Rv2902c_R & - & - & 5'-CTAG $\underline{\text { CTCGAG CTAAGTGCCCTGAGCGCGACC-3' }}$
\end{tabular}

Sequencing Primer

T7_F

$-\quad-$

5'-CCCAGTCACGACGTTGTAAAACG-3'

T7_R

5'-AGCGGATAACAATTTCACACAGG-3'

'F' denotes "Forward" and 'R' denotes "Reverse". The underlined sequences represent the restriction sites at the start of each primer (also see Table 7.8). 


\subsection{Chemical Compound Libraries}

\subsubsection{Chemical Libraries}

A number of compound libraries have been used in this study in order to identify potential growth inhibitors of the MTBC (section 2.6.2). Five drug libraries were used for the identification of mycobacterial growth inhibitors.

\section{A) LOPAC Library ${ }^{1280} \mathrm{TM}$}

LOPAC $^{1280}$ stands for Library of Pharmacologically Active Compounds (Figure 2.1). An entire collection of LOPAC was purchased from Sigma Aldrich Company and consists of 1280 distinct compounds, with a wide range of biological targets. As the name of this library indicates, compounds in this library are already pharmacologically active; therefore, it was expected to yield compounds that were able to inhibit mycobacterial growth to optimise the screening procedure.

\section{B) Spectrum Collection}

The Spectrum Collection was purchased and imported from MicroSource Discovery System, Inc (U.S.A). This library had a range of active compounds, and the library consisted of 2,000 compounds in total. There was an overlap of a few compounds in Spectrum library from LOPAC, which therefore, served as embedded controls during the high through screening (HTS). The Spectrum Collection has a wide range of compounds and natural products with biological activities. Potential hits identified from the HTS using the Spectrum library are discussed in Chapter 3. 


\section{C) NIH Diversity Set}

The NIH Diversity Set was imported from the National Institutes of Health (NIH). It contained 1990 compounds in total, to which, a set of natural products was also included (refer below).

\section{D) NIH Natural Product Set}

The NIH Natural Product Set contained about 240 compounds from natural sources. These compounds were screened against $M$. bovis $\mathrm{BCG}$ and $M$. tuberculosis $\mathrm{H} 37 \mathrm{Ra}$ to identify novel growth inhibitors. Individual compounds that proved active in the HTS (and that were permitted to be imported into New Zealand by MAF, Biosecurity Department) were then obtained from overseas, by completing an NIH request form (available at http://dtp.cancer.gov/RequestCompounds/index.jsp) through their official website of the NIH, Developmental Therapeutics Program (NIH-DTP).

\section{E) Library of Plant Extracts from NZ Native Plants}

A library of New Zealand native plant extracts was collected and prepared to identify potential anti-mycobacterial activity they might have. Plant samples were collected across the country mainly from Karori Wild-life Sanctuary (Zealandia), Otari-wilton Bush and other suburbs including South Island by [250]. After sample collection, a mini plant extract library was prepared by dissolving extracts in various solvents i.e. methanol, ethanol and aqueous extracts (depending on their solubility) before they were tested for their medicinal activity (section 3.3.1.4). Figure 2.1 shows the number of libraries and compounds used to screen in high-throughput. 


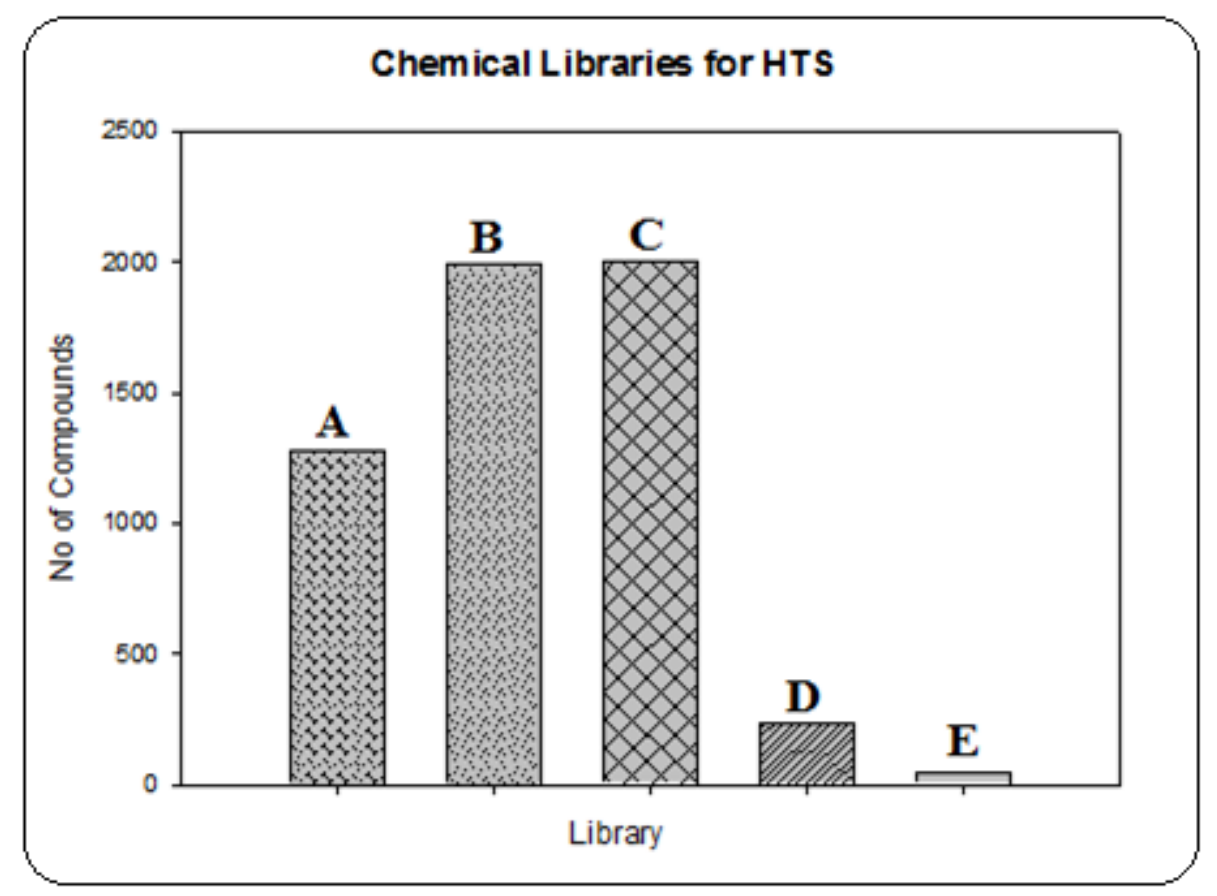

Figure 2.1: Chemical libraries used for high-throughput screening. (A) LOPAC $(n=1280)$ (B) The Spectrum Collection ( $\mathrm{n}=2000)$ (C) The NIH Diversity set $(\mathrm{n}=1990)$ (D) Natural Product set $(\mathrm{n}=240)$ and (E) New Zealand plant extracts $(n=50)$.

\subsubsection{Plate Map for High Throughput Screening}

The first column of the standard 96 -well plate was filled with $150 \mu \mathrm{L}$ of sterile water in order to reduce evaporation of cultures from the plate during long incubation periods. The last column had a range of controls in duplicate (section 3.1.3.2), which were included in each plate used for HTS. The top two wells at position A12 and B12 had media controls (negative control), position C12 and D12 had the $100 \%$ growth controls (cells+media only), position E12 and F12 had 2\% DMSO controls and the last two wells at position G12 and H12 had the antibiotic controls (positive controls), including rifampicin (RIF) and isoniazid (INH), respectively (Figure 2.2). 


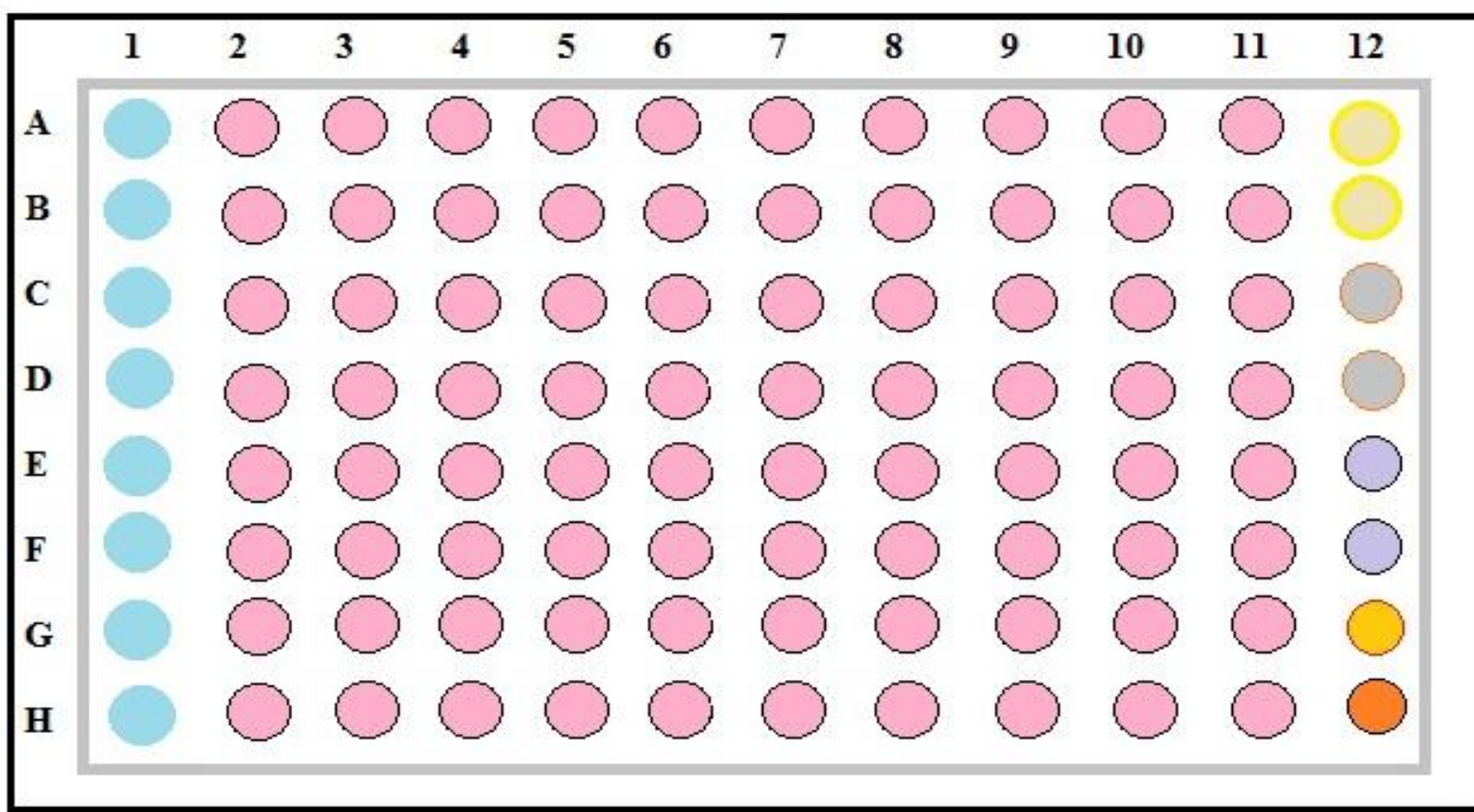

media, media + cells,

$\bigcirc \rightarrow$ solvent control (DMSO) $\mathrm{dH}_{2} \mathrm{O}$ antibiotic control (INH)<smiles>[14CH3][14CH2]c1ccccc1</smiles><smiles>ClCCOCC#CCc1ccccc1</smiles>

Figure 2.2: Plate map used for high-throughput screening. Column 12 shows the arrangement of controls used. A total of 80 compounds (represented as pink circles) were screened per plate and all screens were performed in triplicate. Each compound was dispensed at $20 \mu \mathrm{M}$ final test concentration along with positive controls, rifampicin and isoniazid.

\subsubsection{Individual Compounds for Validation Experiments}

Potential growth inhibitors of $M$. tuberculosis identified from HTS were imported from the NIH (U.S.A) as well from Sigma Aldrich (New Zealand) in order to confirm their antimycobacterial activity in dose-response assays. 


\subsubsection{NIH Chemical Compound Repository}

Unless otherwise stated, all individual chemical compounds obtained for validation experiments were suspended in DMSO. All compounds from the NIH library had a unique 'NSC' ID number.

\subsubsection{96-well Plates for Assay}

For all growth inhibition assays (section 2.7.2.1), dormicidal assays (section 2.7.3), resazurin reduction assays (section 2.7.4), and cytotoxicity assays (section 2.7.5), standard roundbottom 96-well plates were used (BD Falcon ${ }^{\mathrm{TM}}$ ).

\subsubsection{Sigma Plot for Data Analysis}

Unless otherwise stated, all data produced from growth inhibition assays, dormicidal and cytotoxicity assays were analysed with Sigma Plot Software (SYSTAT) version 11.0 to generate four-parameter logistic standard curves. 50\% inhibitory and minimum inhibitory concentrations were determined using standard curves.

\subsubsection{Dose-Response Assays}

The plate map for dose response assays was the same as the map presented for HTS (Figure 2.2) except for the arrangement of controls (Figure 7.12).

\subsubsection{Plate Map for Dose-Response Assays}

The set up for various dose-response and bacteriostatic assays was slightly different from the HTS format in terms of the arrangement of controls (Chapter 3). For all assays other than HTS, in the first row and $8^{\text {th }}$ row from wells A1-A12 and H1-H12, respectively, $150 \mu \mathrm{L}$ of 
sterile water was added to reduce evaporation of the medium. In the first column (A1-H1), sterile 7H9 medium (without antibiotics and cells) was added as negative controls for bacterial growth. The last column (right-hand side) wells B12 and D12, 100\% growth (medium + cells) controls were added and a standard first line anti-Tb drug either rifampicin or isoniazid was included in triplicate as a positive control for the assay at well position numbers E12 to G12 (Figure 7.12).

\subsubsection{Novelty of Inhibitors}

For novel inhibitors identified from the HTS part of this study (Chapter 3), pre-set criteria were followed for selecting compounds for further characterization and data analysis. These selection criteria were based on two approaches, experimental approach and a literature-based approach (section 3.3.1.1.4).

\subsubsection{Import of Anti-HIV RNase H Compounds}

More than 50 HIV RNase $\mathrm{H}$ inhibitors were selected from the PubChem database and a list was prepared after prioritising all compounds on the basis of their specific activity (i.e. active against HIV but no inhibitory activity against human RNase H). All compounds were then imported from the NIH (U.S.A).

\subsubsection{Structure-activity Relationships}

A good structural and practical analysis of all compounds selected in an early discovery programme can be time and cost efficient. Chemical structures of prioritised compounds both in terms of high anti-mycobacterial activity and low cytotoxicity towards human cells were compared for structural similarity with currently in-use anti-tubercular drugs. An initial 
structural comparison was performed to examine if such compounds had different structures and were likely to have a novel mechanism of action.

\subsubsection{Lipinski's Rules to Evaluate Drug-like Properties of Lead Compounds}

Christopher Lipinski has described rule-of-5 to evaluate the likelihood of lead compounds to be developed into drugs [251]. These rules state that an ideal drug will have a low molecular weight (less than 500 Daltons) for better solubility and absorption. Compounds should have fewer rings with a smaller number of rotatable bonds. After following our selection criteria (section 3.3.1.1.4 and section 5.3.4), Lipinski's rule-of-5 was applied on compounds identified and selected for validation to confirm if these inhibitors had potential to be developed into drugs (section 5.7), as it was deemed appropriate to perform a brief comparison of compounds identified in this study with the available anti-tubercular drugs and to determine if they violated any of the rules (Chapter 5). Following that, structural analogues of active compounds were imported for validation experiments. 


\subsection{Methods}

\subsubsection{Tuberculosis Screening Models - M. bovis BCG and M. tuberculosis H37Ra}

A slow-growing member of the MTBC and a Tb vaccine strain, $M$. bovis $\mathrm{BCG}$ (Table 2.1) was employed for screening of compound libraries. After preliminary screening assays, inhibitors were validated against another surrogate species, M. tuberculosis H37Ra. Both strains are slow-growing species and have a doubling time of 18-24 hrs. A fast-growing mycobacterial species, M. smegmatis $\mathrm{mc}^{2} 155$ was also used in this work to compare screening data received from various Tb models (Chapter 4).

\subsubsection{Growth Inhibition Assays for Mycobacteria}

\section{Growth Inhibitory Assay}

In a growth inhibitory assay, the percentage inhibition (or alternatively, a measurement of cell viability) of bacterial growth is determined for any active extract, inhibitor, chemical compound, or drug. The growth inhibition assay is also referred to as a bacteriostatic assay (to determine the activity of unknown compounds which are responsible for bacterial growth inhibition). Section 2.7.2 details the methodology of the bacteriostatic assays.

\subsubsection{Bacteriostatic Assay for M. bovis BCG}

Unless otherwise stated, all M. bovis BCG cultures were grown in 7H9 medium enriched with OADC as described (section 2.2.1). After initial culture inoculation, both $M$. bovis BCG and M. tuberculosis attain their log phase growth in about 2-3 days. Both species tend to make clumps of colonies during growth, which significantly affects assay readings, especially when reading optical density for cultures. Therefore, an $\mathrm{OD}_{600}$ and GFP fluorescence correlation were used to analyse the data from inhibitory assays (section 3.2.2). Tween80 was used in all 
assays as a surfactant to facilitate bacterial growth with concentrations between $0.025-0.05$ percent; it causes emulsification and restricts cells adhesion. Shaking is another way to avoid bacterial clumping, which helps keep bacterial colonies a part; therefore, a mild sonication of mycobacterial cultures was performed before cells were seeded into an assay. Midlogarithmically growing $M$. bovis BCG and M. tuberculosis $\mathrm{H} 37 \mathrm{Ra}$ cultures were used in all growth inhibition assays. Cells were used by diluting the culture down to an $\mathrm{OD}_{600}$ of 0.05 (10 mm path length glass cuvettes).

A two-fold whole plate dilution series of standard anti-Tb drugs was performed in triplicate. Depending on the MICs of the known anti-tubercular drugs published in the Handbook of Anti-Tuberculosis Agents [178], different drugs were added at starting concentration of at least 3-4 times higher than their MIC values in order to observe complete inhibitory concentrations and to determine a range of inhibitory concentrations for calculating a concentration that inhibits growth by $50 \%\left(\mathrm{IC}_{50}\right)$. Fluorescent cultures of $M$. bovis $\mathrm{BCG}$ were diluted to an optical density of 0.1 at $\mathrm{A}_{600} \mathrm{~nm}$, and $50 \mu \mathrm{L}$ of a diluted cell culture was added to the drug-containing wells. All cultures were seeded at a final $\mathrm{OD}_{600}$ of 0.05 . For the negative controls, sterile 7H9 medium was added without antibiotics and for positive controls, known standard drugs were added at a final concentration of $20 \mu \mathrm{M}$ along with $50 \mu \mathrm{L}$ cells (with final $\mathrm{OD}_{600}$ of 0.05). Details of the arrangements of controls and the use of test compounds are presented in the plate map for dose-response assays in figure 7.12. All assays were performed in 96-wells plates, and the final volume for all assays was $100 \mu \mathrm{L} /$ well. Solvent controls (cells and medium only) were also included to compare 100\% M. bovis BCG growth to drug-treated positive controls. All 96-well plates were covered with lids, sealed with a thin-seal cellulose film and wrapped again with parafilm to reduce media evaporation over long incubation time periods. A after complete labelling, all plates were placed in specific polythene bags and were stacked in a shaking incubator (DK-S1020, Global Science) 
and incubated at $37{ }^{\circ} \mathrm{C}$ for 2 weeks with constant shaking of $200 \mathrm{rpm}$. After an appropriate

incubation time, all assays were read by 25 point scan $(5 \times 5)$ using a Perkin-Elmer Envision ${ }^{\mathrm{TM}}$ Multi-Label Plate Reader for the measurement of optical density at $600 \mathrm{~nm}$ and the GFP signal (excitation $490 \mathrm{~nm}$ and emission $510 \mathrm{~nm}$ ). The final results were based on the correlation of both $\mathrm{OD}_{600}$ and GFP fluorescence for $M$. bovis $\mathrm{BCG}$. $\mathrm{IC}_{50}$ values (defined as the concentration of drug that inhibits fluorescence of the cultures by $50 \%$ compared to the no-drug positive controls) of all drugs tested were calculated through SigmaPlot version 11.0.

\subsection{Assay for M. tuberculosis}

All growth conditions and assay time points for M. tuberculosis H37Ra were exactly the same as for $M$. bovis BCG except that BCG harboured a pYL_gfp plasmid (Table 2.2) carrying GFP and kanamycin resistance $(50 \mu \mathrm{g} / \mathrm{mL})$ genes. In contrast, M. tuberculosis H37Ra did not have a GFP gene and therefore, results for validation of M. tuberculosis inhibitors are based on $\mathrm{OD}_{600}$ only. The $\mathrm{OD}_{600}$ values were comparable to GFP values obtained from the M. bovis BCG. Figure 7.12 presents the arrangement of wells for the growth inhibition assay in M. tuberculosis H37Ra. Novel anti-Tb compounds detected in the present study were also tested against the virulent strain of $\mathrm{Tb}$, M. tuberculosis $\mathrm{H} 37 \mathrm{Rv}$ at Johns Hopkins University, Baltimore (U.S.A) with similar assay protocol as described (section 2.7.2).

\subsubsection{Bacteriostatic Assay for Mycobacterium smegmatis $\mathbf{m c}^{2} 155$}

While M. smegmatis is fast growing mycobacterial species, which divides after every 2-3 hours, it does not require special growth medium requirements compared to $M$. bovis BCG or M. tuberculosis H37Ra (section 2.2.1). Therefore, LB medium with or without antibiotics was used to culture $M$. smegmatis both on liquid as well as on solid agar plates. However, 
when comparison of various $\mathrm{Tb}$ models was carried out to evaluate the efficacy of each model to pick up M. tuberculosis-specific inhibitors (Chapter 4), enriched medium was used, the same as used for M. bovis BCG and M. tuberculosis H37Ra (described in section 2.7.2.1) to keep the growth conditions identical across all species. M. smegmatis cells were diluted down to an $\mathrm{OD}_{570}$ of 0.1 , and the final volume was $100 \mu \mathrm{L}$ per well (as described in section 2.2.2). Inhibitors were tested for bacteriostatic activity using $M$. smegmatis as previously described [252].

\subsubsection{Bacteriostatic Assay for Non-mycobacterial Clinical Strains}

Various non-mycobacterial clinical strains were used in this study (Table 2.1) to test the spectrum of mycobacterial inhibitors across a range of other clinically important bacterial species. Assay set-up for these strains was not different from M. smegmatis (section 2.7.2.2). However, media conditions (section 2.2.3) and time points were different. After challenging with drugs/compounds, all plates were left in the incubator at $37{ }^{\circ} \mathrm{C}$ for overnight incubation. Appropriate controls were included in each plate. Assays were read at $570 \mathrm{~nm}$ wavelength for their optical density and for resazurin readings as discussed in section 2.7.5 using the Perkin Elmer ${ }^{\mathrm{TM}}$ Multi-label plate reader.

\subsubsection{Colony Forming Units for Mycobacteria}

\subsection{CFU's for M. tuberculosis}

The optical density of pure M. tuberculosis cultures was checked at $\mathrm{OD}_{600}$ in $1 \mathrm{~mL}$ (section 7.2.1), and then culture was serially diluted in PBS (10-fold for 7 times) before plating them out in order to calculate the viable number of cells in each millilitre of $M$. tuberculosis culture (CFU's/mL). An aliquot of each dilution $(100 \mu \mathrm{L})$ was plated at least in duplicate on $7 \mathrm{H} 10$ 
agar plates supplemented with $10 \%$ filter sterilized OADC (section 2.2.1.1) and $0.5 \%$ glycerol (with or without $0.1 \%$ Tween80) in a sterile environment. All plates for $M$. tuberculosis CFUs were wrapped tightly in parafilm and then with aluminium foil, and then the plates were incubated for $3-4$ weeks at $37{ }^{\circ} \mathrm{C}$ before individual colonies were counted, each colony representing viable $M$. tuberculosis cell. The counted CFUs were also used to estimate the number of viable cells present in $1 \mathrm{~mL}$ of original $M$. tuberculosis culture.

CFUs/mL $=\mathrm{N}^{\mathrm{o}}$ of individual colonies $\mathrm{x}$ dilution factor $\left(10^{\mathrm{n}}\right)$

$0.1 \mathrm{~mL}$ (volume plated)

\subsection{CFU's for M. smegmatis}

The technique of counting CFUs for M. smegmatis was the same as for M. tuberculosis except for the medium and reading time points. After having serially diluted original culture, $0.1 \mathrm{~mL}$ aliquot of the dilutions were aseptically plated on LB agar, and after carefully wrapping, the plates were incubated at $37{ }^{\circ} \mathrm{C}$ in a non-shaking incubator for 96 hours before counting the CFUs.

\subsubsection{Dormicidal Assay Used for Mycobacteria}

A dormicidal assay assesses the death of dormant mycobacterial cells caused by a compound. Novel inhibitors identified in this study were further tested to confirm the type of activity they had. The dormicidal assay confirmed whether anti-mycobacterial compounds identified under nutrient-rich conditions were also active against non-replicating and low metabolically active mycobacteria. $M$. smegmatis_pSHIGH_hsp60 was used in these assays. All cultures were grown for at least 10 days to ensure that cells had reached their stationary phase. Cells were tested for their optical density by centrifuging (CR3i Multifunction Centrifuge, Thermo 
Scientific) and resuspending the pellet to an $\mathrm{OD}_{600}$ of 0.5 in PBS. Cells were washed (three times with PBS) to avoid any medium and nutrient carryovers and resuspended in sterile PBS before they were used in the dormicidal assay. The arrangement of test compounds, media and controls in 96-well plates was same as shown in figure 7.12 except that cells were suspended in PBS instead of 7H9 medium. Carbonyl cyanide m-chlorophenyl hydrazone (CCCP) was used as a positive control to kill all growing and metabolically inactive cells. After 4 days of incubation at $37{ }^{\circ} \mathrm{C}, 30 \mu \mathrm{L}$ of $0.02 \%(\mathrm{v} / \mathrm{v})$ final concentration of resazurin was added to all wells and the plates were re-sealed and further incubated at $37{ }^{\circ} \mathrm{C}$ overnight (Ian Bassett, unpublished method). The data were analysed as described (section 2.6.4) and minimum dormicidal concentration (MDC) and 50\% dormicidal concentration $\left(\mathrm{DC}_{50}\right)$ values were calculated for the test compounds.

\subsubsection{Resazurin Reduction Assay}

Resazurin reduction assay is robust and gives a reliable determination of redox-state of live cells and an easy interpretation of results. Resazurin is an oxidation-reduction indicator used to detect viability of cells [253], and was used in dormicidal assays instead of GFP fluorescence. Resazurin salt is a non- fluorescent to weak fluorescent blue dye which gets reduced to a pink fluorescent dye, indicating the viability of cells (Figure 2.3).
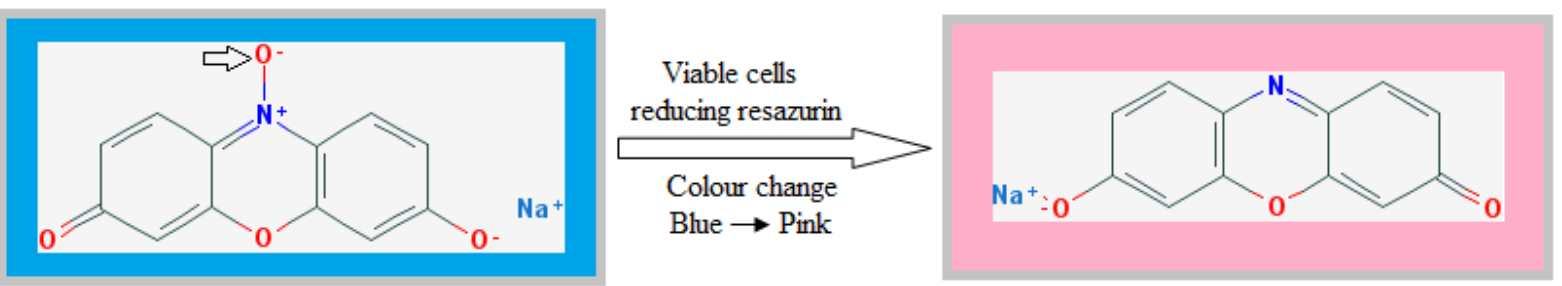

Figure 2.3: Biochemical reduction of resazurin sodium salt into resorufin. The activity of cellular respiration indicates the viability of cells. Resazurin salt (MW $251.18 \mathrm{~g} / \mathrm{mol}$ ) is reduced by removal of one 
oxygen atom (indicated by an arrow), yielding a MW of $235.18 \mathrm{~g} / \mathrm{mol}$ of the fluorescent compound (pink). Chemical structures were retrieved from the PubChem database. MW, molecular weight.

Unlike $M$. bovis BCG, $M$. tuberculosis H37Ra did not have a reporter system to detect cell viability; therefore, a resazurin reduction method was used. Similar assay conditions were adapted for resazurin assay as previously described [254], with slight modifications. At the end of the drug exposure time, $30 \mu \mathrm{L}$ of $0.02 \%(w / v)$ final concentration of resazurin was added to all wells, and the plates were incubated without agitation at $37{ }^{\circ} \mathrm{C}$ overnight for $M$. tuberculosis and for six hours for M. smegmatis. Resazurin gets converted from its blue colour into pink, after being reduced by the mitochondrial enzymes of viable cells (Figure 2.3). Plates were read for fluorescence by excitation and emission at $530 \mathrm{~nm}$ and $590 \mathrm{~nm}$, respectively, in a Perkin Elmer - EnVision ${ }^{\mathrm{TM}}$ Multi-label plate reader using a fluorescein isothiocyanate (FITC) filter.

\subsubsection{Cytotoxicity Assay}

The human leukaemic cancer cell line (HL-60) and the murine macrophage cell line (J774.A1) were used in this study to evaluate the level of toxicity of the novel compounds. All cytotoxicity assays were performed in 96-well plates (Ray-Lab) using a standard tissue culture facility in a sterile environment. The experimental protocol to determine cytotoxicity of compounds in the murine macrophage J774.A1 cell line was used as previously described $[255]$.

\subsubsection{Murine Macrophage J774.A1 Cell Line}

J774.A1 adherent cells were cultured in $250 \mathrm{~mL}$ T-75 flasks (Invitrogen) using the standard recommended RPMI-1640 medium supplemented with 10\% filter-sterilized Fetal Calf Serum 
(FCS), 0.25 Units/mL insulin and a combination of antibiotics, $1 \%$ penicillin-streptomycin (100 Units/mL penicillin and 100 Units/mL streptomycin). Cells were incubated at $37{ }^{\circ} \mathrm{C}$ in a $5 \% \mathrm{CO}_{2}$ air atmosphere $\left(\mathrm{CO}_{2}\right.$ Incubator, SANYO) until cells had become $80 \%$ confluent.

\subsection{MTT Assay and Data Analysis}

Test compounds were added to 96-well plates (black-walled with flat glass bottom from BD Bioscience) and two-fold serial dilution were performed. Cells were then seeded into the required wells at a concentration of $1 \times 10^{5}$ (50 $\mu \mathrm{L}$ of 10,000 cells/well), and the plates were incubated for 72 hours. No compound/drug was added to the control wells in order to observe complete cell growth. MTT (20 $\mu \mathrm{L}$ of $5 \mathrm{mg} / \mathrm{mL}$ solution) [3-(4, 5-dimethylthiazol-2-yl)-2, 5diphenoltetrazolium bromide] (Sigma Aldrich), was added to all wells after three days of incubation. Plates were wrapped in a Gladwrap and incubated for two hours. A $100 \mu \mathrm{L}$ of solubilizer $(10 \%$ SDS in $0.01 \mathrm{~N} \mathrm{HCl})$ was added to each well, and plates were further incubated at $37{ }^{\circ} \mathrm{C}$ overnight and read at $570 \mathrm{~nm}$. All assays were performed in triplicate and data were analysed with Sigma Plot Software (version 11.0) using a four-parameter logistic standard curve analysis to determine $\mathrm{IC}_{50}$ values.

\subsection{Passaging the J774 Cell Line}

For continuous passaging of cells, $400 \mu \mathrm{L}$ of trypsin enzyme was added to $80 \%$ confluent cells. Trypsin incubation at $37{ }^{\circ} \mathrm{C}$ for $5-10$ minutes helps to detach the layer of adherent macrophage cells. Cells were spun down in a $15 \mathrm{~mL}$ centrifuge tube at $300 \mathrm{~g}$ for 5 minutes. The supernatant was discarded, and the cells were resuspended in $5 \mathrm{~mL}$ of fresh medium. Washed cells $(100-200 \mu \mathrm{L})$ were then added to $10 \mathrm{~mL}$ of fresh medium and the passage number was recorded. If further passage was not required, cells were frozen at $1 \times 10^{6}$ 
cells/cryovial in $10 \%$ DMSO in a total volume of $1 \mathrm{~mL}$. Initially, cells were frozen in a -80 freezer overnight then transferred to liquid nitrogen for long-term storage.

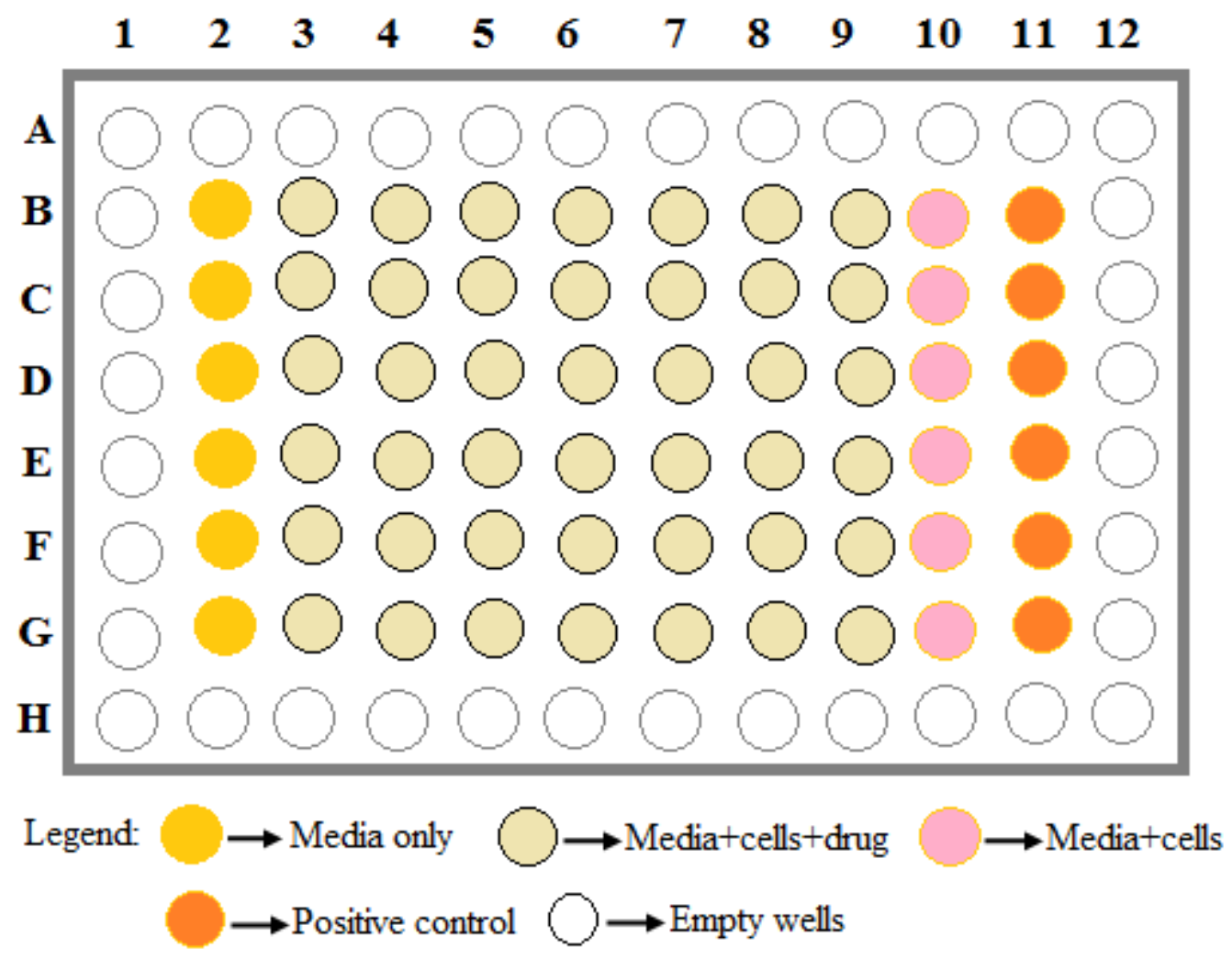

Figure 2.4: Plate map for mammalian cell cytotoxicity assays. From column 3-9, two compounds were tested per plate in triplicate. Outer wells were left empty.

\subsubsection{HL-60 (Human Leukaemic) Cell Line}

Non-adherent human promyelocytic leukaemic cells were grown in $25 \mathrm{~cm}^{2} \mathrm{~T}-25$ flasks (RayLab) using standard RPMI-1640 medium. For HL-60 cells, RPMI media was supplemented by $10 \%$ filter-sterilized FCS along with $1 \%$ penicillin-streptomycin $(100$ Units/mL penicillin and 100 Units $/ \mathrm{mL}$ streptomycin). When the cells were $80 \%$ confluent, $50 \mu \mathrm{L}$ of medium was added to each well of a 96-well plate followed by the test-compounds and two-fold serial dilutions was performed before $50 \mu \mathrm{L}$ of 10,000 cells/well were added to the required wells 
(Figure 2.4). For compounds that were dissolved in DMSO, the highest test concentration for such compounds was kept to $100 \mu \mathrm{M}$, thereby, not exceeding a $1 \%$ final concentration of DMSO. DMSO concentrations of $>1 \%$ inhibit HL-60 cell growth. Plates were incubated at 37 ${ }^{\circ} \mathrm{C}$ for 48 hours in a tissue-culture incubator (Heraeus).

\subsection{Cell Count for Seeding}

To count viable cells in an Improved Neubauer Chamber (Haemocytometer), cells were stained with trypan blue (Sigma Aldrich). Trypan blue was used to differentiate between live and dead cells as it crosses the plasma membrane of dead cells only, leaving the live cells unstained due to an impermeable nature of the cell membrane of live cells. Cells $(10 \mu \mathrm{L})$ were mixed with $10 \mu \mathrm{L}$ of stain (1:1), and then $10 \mu \mathrm{L}$ of this mixture was loaded onto haemocytometer. Cells were counted in the four outer large squares (Figure 2.5). An average of the four large squares was taken, and the number of cells was estimated using the formula $\mathrm{C}_{1} \mathrm{~V}_{1}=\mathrm{C}_{2} \mathrm{~V}_{2}$ to calculate the quantity of cells required from the stocks to make up working suspension of $4-5 \mathrm{~mL}$ of cells $\left(2 \times 10^{5}\right.$ cells $\left./ \mathrm{mL}\right)$.

A $50 \mu \mathrm{L}$ of cells was then added to each well except the blank wells where only $100 \mu \mathrm{L}$ of medium was added. All assay plates were then incubated at $37{ }^{\circ} \mathrm{C}$ without agitation for 48 hours. MTT solution and the absorbance of wells determined as described above in section 2.7.5.1.1. All cytotoxicity assays were performed in Biological Safety Cabinet Class II (AES Environmental Pvt. Ltd.). 


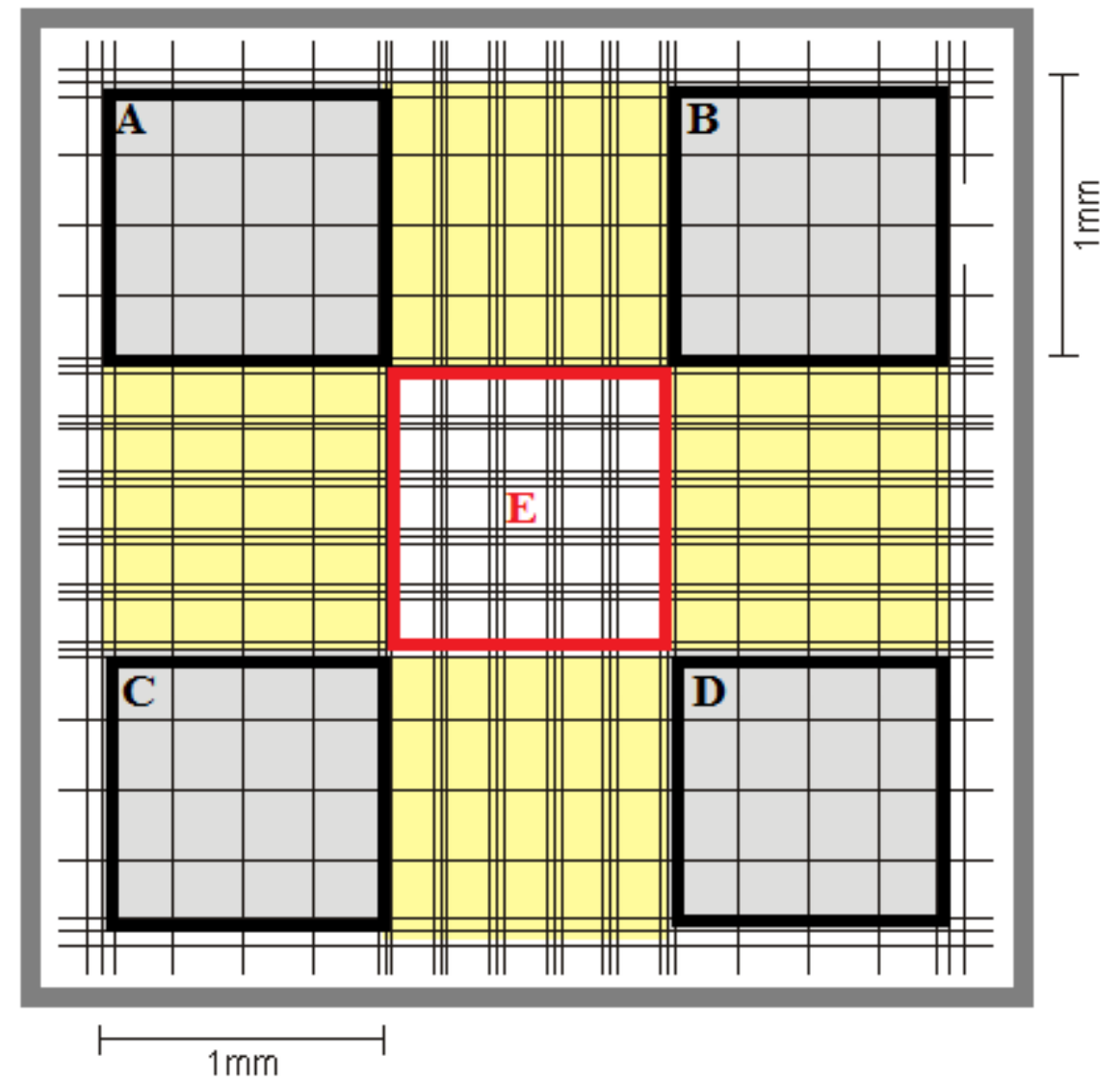

Figure 2.5: Improved Neubauer Chamber (Haemocytometer). HL-60 cells were counted in 4 squares, (A, B, C and D), (black squares); whereas, macrophage cells were counted in (E) the middle 25 squares (within large red square). A total sample volume of $10 \mu \mathrm{L}$ was loaded onto the chamber for cells counting.

\subsection{Controls for Cytotoxicity Assay}

A known mammalian cell cytotoxic compound, epothilone A (Epo A), (Merck) was used as a positive control in the cytotoxicity assays. For observation of normal cell growth, growth controls were included without drug(s) (cells and medium, $50 \mu \mathrm{L}$ each) in each plate. For the medium blank, only medium without cells or drug was added. The arrangement of controls and test compounds used in cytotoxicity assays are presented in figure 2.4 . 


\subsubsection{Cloning of M. tuberculosis Ribonuclease HI/II Proteins}

\section{Transformation of pET28a(+)/pET28a(+)_Rv2228c and Rv2902c into E. coli Strains}

E. coli strains (DH5 $\alpha$ and BL21-DE3) were transformed with various plasmids for the cloning procedures. A heat transformation method was used for most of $E$. coli strains rather than electroporation.

\subsubsection{Calcium Competent $E$. coli Cells}

E. coli cultures from $-80{ }^{\circ} \mathrm{C}$ freezer stocks were streaked on agar LB plates and incubated overnight at $37{ }^{\circ} \mathrm{C}$. Individual colonies were then selected and transferred into $5 \mathrm{~mL}$ of LB broth and further incubated overnight. This $5 \mathrm{~mL}$ of culture was sub-cultured into $50 \mathrm{~mL}$ of medium (1:10) the following day into a sterile $250 \mathrm{~mL}$ flask with a cotton plug on wrapped in aluminium foil. The culture flask was placed in a shaking incubator (with set speed of 200 rpm) at $37{ }^{\circ} \mathrm{C}$ for a few hours until the optical density of the culture at $590 \mathrm{~nm}$ was $\sim 0.5$. $\mathrm{OD}_{590} \mathrm{~nm}$ was read in a spectrophotometer (Thermo Scientific). These logarithmically growing cells were then transferred into $50 \mathrm{~mL}$ polypropylene tube and the tube was placed in ice for at least 25 minutes. Cells were harvested by centrifugation at $4000 \mathrm{rpm}$ for 20 minutes. The supernatant was discarded and a $50 \mathrm{~mL}$ of pre-chilled and sterile $0.1 \mathrm{M}$ calcium chloride $\left(0.1 \mathrm{M} \mathrm{CaCl}_{2}\right)$ was added to the pellets. Cells were then placed back on ice for the next 20 minutes after which they were recovered by centrifugation at $4000 \mathrm{rpm}$ for 10 minutes. This washing step was repeated twice by reducing the amount of $\mathrm{CaCl}_{2}$ by half $(25$ $\mathrm{mL}$ ) in the second wash. Finally, 2-4 $\mathrm{mL}$ of $\mathrm{CaCl}_{2}$ was added along with $14 \%$ glycerol. Cells were thoroughly mixed and $100 \mu \mathrm{L}$ aliquots were dispensed and stored in a $-80{ }^{\circ} \mathrm{C}$ freezer for later use. 


\subsubsection{Heat Shock Transformation}

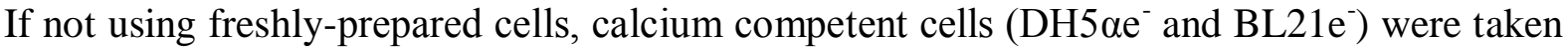
from $-80{ }^{\circ} \mathrm{C}$ freezer stocks and thawed on ice for 10 minutes. The E. coli bacteria were transformed with both pET28a (+) plasmids carrying Rv2228c and Rv2902c sequences along with appropriate transformation controls (section 2.7.6.4). The DNA concentration averaged about $20-30 \mathrm{ng} / \mathrm{mL}$ for each plasmid. Plasmids were taken from $-20{ }^{\circ} \mathrm{C}$ stocks and put on ice for about 5 minutes before $2 \mu \mathrm{L}$ was added to the competent cells and mixed gently and left on ice for 10 minutes. The cells plus plasmid were then put on a heat block for heat shock transformation with a pre-set temperature of $42{ }^{\circ} \mathrm{C}$ and left for 90 seconds. After heat shock, the tubes were placed back on ice for up to 2 minutes. A $900 \mu \mathrm{L}$ aliquot of antibiotic-free $\mathrm{LB}$ medium was added to the transformed cells, and the tubes were placed on a shaker at $200 \mathrm{rpm}$ for an hour to allow the cells to recover and to allow the expression of the kanamycin resistance gene. After this incubation, cells were harvested by centrifugation at $14,000 \mathrm{rpm}$ for 1 minute, the supernatant was discarded and the cells were resuspended in LB medium and gently mixed. A $100 \mu \mathrm{L}$ aliquot of these cells was then plated aseptically onto LB plates with the required antibiotics. The plates were sealed and incubated at $37{ }^{\circ} \mathrm{C}$ overnight in order to select for transformants that expressed the kanamycin resistance gene.

\subsubsection{Electroporation of pHS201 into M. bovis BCG}

Both pSHIGH and pHS201 harboured a GFP gene used to confirm the viability of BCG cells in growth inhibition assays with good reliability. Both these plasmids also have kanamycin resistance genes, $\operatorname{kan}^{\mathrm{r}} 30 \mu \mathrm{g} / \mathrm{mL}$ (Table 2.2). GFP fluorescence was used as a viability indicator of mycobacterial cells and to make comparisons with optical density measurements to get more reliable results. pHS201 plasmid was electroporated into $M$. bovis BCG with Gene Pulser Xcell ${ }^{\mathrm{TM}}$ (BIO-RAD). Plasmid preparations were cleaned using a plasmid clean 
kit and DNA was quantified using a NanoDrop® - ND100 Spectrophotometer (NanoDrop Technologies, Auckland, New Zealand) (Table 7.7). Electrocompetent cells were thawed on ice for 5 minutes, and then $200 \mu \mathrm{L}$ of $10 \%$ glycerol was added to the cells. Then, $7 \mu \mathrm{L}$ of DNA was added to the aliquot containing the cells and glycerol, and the mixture was kept on ice for 5 minutes. All contents were thoroughly mixed and transferred into a pre-chilled electroporation cuvette. Cells were then placed on ice for 1-2 hours before proceeding with the electroporation step. As per settings described in mycobacteria protocols [256], cuvettes containing cells, DNA and glycerol were electroporated at the following settings;

- Voltage $1250 \mathrm{~V}$

- Capacitance $25 \mu \mathrm{F}$

- Resistance $1000 \Omega$

- Cuvette $2 \mathrm{~mm}$ pathlength

A time constant of 19.7 was recorded for this procedure (well within a range of 15-25 as being successful electroporation). pHS201 plasmid served as a positive control and Milli- $\mathrm{Q}^{\circledR}$ water was used as a negative control for electroporation. After electroporation, cells were transferred into $1 \mathrm{~mL}$ of $7 \mathrm{H} 9$ and incubated with gentle shaking at $37{ }^{\circ} \mathrm{C}$ for at least 48 hours before cells were plated out on 7H10 agar plates (supplemented with OADC) containing kanamycin $50 \mu \mathrm{g} / \mathrm{mL}$. The plates were incubated at $37{ }^{\circ} \mathrm{C}$ for $3-4$ week to select plasmidcontaining colonies. The resulting colonies were further grown in $7 \mathrm{H} 9$ and were confirmed for fluorescence using a FITC filter in an Olympus microscope (Provis AX 70TRF Fluorescent) microscope. Colonies yielding the best fluorescence were used to make freezer stocks in $20 \%$ glycerol final concentration for future use. 


\subsubsection{Transformation Controls}

Both positive and negative controls were included in all transformation experiments. Unless otherwise stated, pCG79 plasmid (Figure 7.1-A) with $20 \mathrm{ng} / \mu \mathrm{L}$ of DNA (Table 2.2) was used as a positive control in all transformation assays, which yielded many individual colonies indicating a successful transformation. For negative controls, only $2 \mu \mathrm{L}$ of sterile water rather than DNA was used which produced no colonies on $\mathrm{kan}+(50 \mu \mathrm{g} / \mathrm{mL})$ plates.

\subsubsection{PCR Reaction Mix}

Rv2228c and Rv2902 from M. tuberculosis H37Rv (encode for RNase HI and HII respectively) were cloned into E. coli expression vector pET28a (+) (section 5.4.6). PCR was performed to amplify the required $M$. tuberculosis genes. All reactions were made on ice and mixed well before loading on to PCR thermal cycler, MJ Mini ${ }^{\mathrm{TM}}$ and My Cycler thermal cycler (BIO-RAD). For a standard $50 \mu \mathrm{L}$ PCR reaction, reagents were used in following order and quantities:

- $\quad$ DEPC $\mathrm{dH}_{2} \mathrm{O}$..............18 $\mu \mathrm{L}$

- $\quad$ DMSO ........................1 $\mu \mathrm{L}$ of $2 \%$

- $\operatorname{Primer}(\mathrm{F}) \ldots \ldots \ldots \ldots \ldots \ldots . \ldots 1 \mu \mathrm{L}$ of $10 \mu \mathrm{M}$

- $\operatorname{Primer}(\mathrm{R}) \ldots \ldots \ldots \ldots \ldots \ldots \ldots \ldots$. $1 \mu \mathrm{L}$ of $10 \mu \mathrm{M}$

- $\quad$ DNA template ...............2 $\mu \mathrm{L}$

- $\mathrm{MgCl}_{2} \ldots \ldots \ldots \ldots \ldots \ldots \ldots . .2 \mu \mathrm{L}$ of $50 \mathrm{mM}$

- Master Mix (Bio Line) ......25 $\mu \mathrm{L}$ 


\subsubsection{Confirmation of PCR Product through Restriction Digestion}

Since there are two natural restriction sites present in the R2228c DNA sequence i.e. BamHI and HindIII (Table 7.8). BamHI and HindIII restriction enzymes were used to perform restriction digestion to initially confirm the right amplification of Rv2288c. The amplification of product was later confirmed by DNA sequencing of PCR products by Macrogen, North Korea (section 2.7.8.2).

pET28a (+) plasmid was extracted from the DH5 $\alpha-80{ }^{\circ} \mathrm{C}$ freezer stocks in order to proceed with the cloning experiments. Restriction digestion was performed on both amplified genes. The same restriction enzymes were used in double digestion experiments. NEB double digest enzyme finder was used to find appropriate conditions and buffer types available at http://www.neb.com/nebecomm/DoubleDigestCalculator.asp. NEB buffer 4 is compatible with both restriction enzymes used in this study, i.e. EcoRI and XhoI.

\subsubsection{Restriction Digestion}

All restriction digestion reaction were prepared either with a total volume of $10 \mu \mathrm{L}$ or $20 \mu \mathrm{L}$. Samples were incubated at $37{ }^{\circ} \mathrm{C}$ overnight, then gel electrophoresis was carried out to confirm a successful restriction by visualizing the correct size bands on a $0.8 \%$ agarose gel under UV light. The materials used and required to perform the restriction digestion along with their respective concentrations are tabulated in table 7.3.

All restriction digests provided with cohesive-end DNA fragments that were suitable for subsequent ligation reactions. 


\subsection{Restriction Digestion Reaction}

Restriction digestion of both vector and PCR products were performed with similar restriction enzymes and conditions used for these experiments (Table 7.4).

\subsection{Heat Inactivation of Restriction Digest}

To halt the restriction enzyme activity to prevent DNA digestion, the reaction was incubated at $65{ }^{\circ} \mathrm{C}$ for at least 20 minutes, as recommended by New England Bio Labs (NEB enzymes, section 2.7.7.2).

\subsection{DNA Fragments Clean Up}

All resulting DNA fragments produced from either restriction digestion or from PCR reaction, were cleaned and concentrated with a PCR Purification Kit (Pure Link ${ }^{\mathrm{TM}}$, Invitrogen) in order to purify the DNA by removing dNTPs, excessive salts and unwanted buffers etc.

\subsubsection{Ligation of Inserts into Vector}

After restriction digestion of amplified PCR products and plasmid, ligation was carried out as follows;

\subsubsection{Ligation Reaction}

For a $20 \mu \mathrm{L}$ ligation reaction, a vector to insert molar ratio was first calculated in order to decide on the specific quantity to be used to set up a ligation reaction. A 1:3 molar ratio of vector-to-insert (insert $30 \mathrm{ng} / \mu \mathrm{L}$ and vector $20 \mathrm{ng} / \mu \mathrm{L}$ DNA concentrations) was calculated on the basis of their DNA concentration. Reaction conditions are given below in order of the reagent addition for setting up a reaction. 
- Buffer (4) $2.0 \mu \mathrm{L}$

- $\mathrm{ddH} 2 \mathrm{O}$ $.8 .5 \mu \mathrm{L}$

- Insert $5.0 \mu \mathrm{L}$

- Vector $.2 .5 \mu \mathrm{L}$

- ATP $1.0 \mu \mathrm{L}$

- $\quad$ T4 DNA ligase ...1.0 $\mu \mathrm{L}$

The vector used in this work was pET28a (+) (Figure 7.1-B) and the insert was Rv2228c from M. tuberculosis for the expression of RNase HI protein in E. coli. The ligation reaction was incubated at $16{ }^{\circ} \mathrm{C}$ overnight before proceeding with the transformation experiments.

\subsubsection{Inactivation of T4 DNA Ligase}

T4 DNA ligase enzyme after ligation was inactivated by heating the reaction to $65{ }^{\circ} \mathrm{C}$ for 20 minutes.

\subsubsection{Ligation Controls}

Along with pET28a (+)_Rv2228c transformation into competent BL21 E. coli cells, as a control, pET28a (+) without insert was also transformed in order to verify a successful ligation experiment before confirmation by restriction digestions and gels electrophoresis. A restriction digestion experiment was performed using EcoRI and XhoI restriction enzymes, followed by running a $0.8 \%$ gel to confirm that the plasmid was cut at the right position and has released the insert of correct size (Figure 5.9). 


\subsubsection{Confirmation of Ligation Reaction}

Ligation reactions were confirmed for inserts performing overnight double digestion with the same set of restriction enzymes. Agarose gel electrophoresis was done to confirm if ligation experiments was successful by visualizing the right size bands under UV light. Restriction digestion of pET28 (+)_Rv2228c was performed as per conditions given in table 7.4.

\subsubsection{Agarose Gel Electrophoresis}

Agarose gels (1\%) were prepared by dissolving one gram of molecular grade agarose into $100 \mathrm{~mL}$ of $1 \mathrm{x}$ TAE. The agarose was melted in a laboratory microwave, and after a few minutes of cooling, $5 \mu \mathrm{L}$ of $10 \mathrm{mg} / \mathrm{mL}$ ethidium bromide was added into $50 \mathrm{~mL}$ of agarose gel. The solution was mixed well and poured into a gel caster in a pre-set electrophoresis assembly (Bio Rad). Appropriate gel combs were placed into the gel, and it was allowed to set at room temperature for at least 20 minutes. DNA samples were mixed with 5x DNA loading buffer (1:4) and loaded along with $5 \mu \mathrm{L}$ of a DNA ladder to estimate the size of the DNA bands. Gels were run according to the conditions detailed in table 7.5; and after the separation of bands, DNA was visualized in an Ultraviolet-Transilluminator (EL Logic 100 IMAGING SyStEM). Gel photos were taken using a Gel Documentation System with high performance KODAK $1 \mathrm{D}^{\mathrm{TM}}$ wide-zoom digital camera. Photos were printed using a Digital Graphic Printer, UP-D895 (Sony).

\subsubsection{Identification of Recombinant Clones}

After confirmation of the correct inserts via restriction analysis (section 2.7.7.4), the plasmids were transformed into the BL21 strain and grown overnight at $37{ }^{\circ} \mathrm{C}$ with kanamycin 50 $\mu \mathrm{g} / \mathrm{mL}$. The plasmid was then extracted from these cultures with a plasmid extraction kit 
(High-speed Plasmid Mini Kit, Geneaid Biotech Ltd.). Recombinant clones were identified by a colony PCR method (section 2.7.8.1).

\subsubsection{Colony PCR}

Individual colonies were picked up by micropipette tip dipped into PBS and were placed in a PCR reaction as a DNA template. PCR was run as per conditions presented in table 7.2 using appropriate primers (Table 2.4). The PCR screen confirmed the correct inserts, after amplification of the desired genes from M. tuberculosis, and confirming correct sizes on a $1 \%$ agarose gel (for Rv2228c, 1095 bp and Rv2902c, 795 bp). Plasmids carrying the genes of interest were grown in $\mathrm{DH} 5 \alpha$ to make $-80{ }^{\circ} \mathrm{C}$ freezer stocks, and the desired clones were selected for further analysis.

\subsubsection{DNA Sequencing}

All samples for DNA sequencing (PCR products, primers, and DNA samples for confirmation of orientation of correct inserts) were sent to the Macrogen DNA sequencing facility in North Korea. All samples were sent either in $200 \mu \mathrm{L}$ PCR tubes or $1.5 \mathrm{~mL}$ eppendorf tubes, which contained PCR products to reconfirm the fidelity of the DNA polymerase enzyme and primers. The amount of DNA for plasmids was about $200 \mathrm{ng}(20 \mu \mathrm{L})$ and for PCR products $50 \mathrm{ng}(10 \mu \mathrm{L})$, for primers 5.0 pmol taken from a $10 \mu \mathrm{M}(5 \mu \mathrm{L})$ stock. All DNA sample containing tubes were wrapped with bubble paper to avoid possible sample damage and to ensure safe transportation of DNA samples. Chromatogram files were studied using Chromas (Version 2.3) and vector NTI Advance ${ }^{\circledR} 11.0$ software. 


\subsection{In Silico Analysis}

In silico analysis was performed to predict the interaction of novel anti-Tb compounds with RNase HI in $M$. tuberculosis (section 5.5). A number of docking software were used to examine if the interaction of previously-known HIV RNase $\mathrm{H}$ inhibitors with mycobacterial RNase HI (Rv2228c) is possible (section 5.5). These include QuacPac (version 1.3.1), Omega (version 2.4.3), FRED (2.2.5), VIDA (version 4.0.3) and OpenEye Scientific Software, Inc. Santa Fe, NM (U.S.A, available at www.eyesopen.com, 2010). 


\section{Chapter 3}

High Throughput Screening of Chemical Libraries for the Identification of M. tuberculosis Inhibitors 


\subsection{Introduction}

\subsubsection{Purpose}

Tb continues to be a serious health concern [100], [257] owing to its unmet needs for drug discovery [258]. Apart from drug resistant $\mathrm{Tb}$ [259], the magnitude of the HIV-Tb coinfection problem is remarkably huge, jeopardising the Tb control efforts worldwide [260]. It has been estimated that without proper containment, treatment and control programs, billions more will be infected and about millions of lives will be claimed by 2020 [261]. It is therefore necessary to find new drugs to strengthen $\mathrm{Tb}$ treatment regimen [262]. One of the effective ways of searching new drugs is random screening of chemical libraries to identify novel lead compounds [263].

\subsubsection{High Throughput Screening}

While there is no preferred method to identify potential anti-tubercular compounds, high throughput screening (HTS) can discover novel leads with high probability [264]. HTS is a process that screens thousands of chemical or drug-like compounds in a relatively short period of time to select ideal candidates with good pharmacological potential. Once identified, these can be further characterized and modified to develop a highly active anti-mycobacterial drug. Broth macrodilution methods and plating colony forming units (CFUs) are traditional orthodox techniques to test compounds for their efficacy and antimycobacterial susceptibility, however, both these methods are time consuming and labour intensive. With the advancement of science and technology, new ways to discover inhibitors against various pathogenic species in a time-efficient manner have been developed. One such example is the development of drug libraries with a collection of compounds from a range of sources. These libraries are batch-screened against the desired microbes to identify potential growth 
inhibitors. The process of screening drug libraries in high-throughput makes it even more convenient and time efficient. Thousands of compounds can be tested at a time against the organism of interest; hence HTS plays a pivotal role in drug discovery. Scientific and drug discovery research relies on HTS for accelerating the drug discovery process [265-267]. Section 3.1.2.1 describes the types of screening procedures for drug discovery.

\subsubsection{Types of Screens}

In vitro high throughput screen can be performed in various ways with their own advantages and limitations. Two main types of screens used for the identification of random and targetspecific inhibitors are discussed below;

\section{A. Targeted-based screening}

When cell-free and targeted-based screenings are performed against an array of biological targets, cell-free assays will only inhibit specific cellular processes. The approach of screening for specific enzyme inhibitors for drug development and its structure-activity relationships is well-known and documented [268-272]. However, target-based screenings have a limitation of selecting inhibitors with only one target at a time. In contrast, whole cell-based screens can detect inhibitors with a wide range of targets, which may have novel modes of action.

B. Whole cell-based high throughput screening

There are a number of advantages for using cell-based assay rather than cell-free assays for compound screening that include cost effectiveness, convenience and robustness [273-274]. Cell-based in vitro screening is a preferred method for the identification of active M. tuberculosis inhibitors [275-276]. A whole cell-based 
screening approach was therefore selected in this study to perform a primary screen to detect novel M. tuberculosis inhibitors. The rationale for using a whole cell-based screen was to perform a random screen to identify mycobacterial inhibitors with multiple-targets in M. bovis BCG, which could then be validated in M. tuberculosis.

\subsubsection{Chemical Libraries for HTS}

The main objective of this part of the study was to perform random high-throughput screening against $M$. bovis BCG using five chemical compound and plant extract libraries (Figure 2.1 and section 3.3.1).

\subsubsection{Stock Concentration of Compound Libraries}

Unless otherwise stated, all libraries were imported with concentrated stocks in deep 96-well plates (10 mM stock concentration). Thereafter, chemical libraries were diluted (10-fold) to 1 $\mathrm{mM}$ using DMSO in order to prepare working stocks. A $2 \mu \mathrm{L}$ aliquot of these working stocks were then dispensed into wells of a 96-well plate with a final concentration of $20 \mu \mathrm{M}$ for each compound tested. Libraries were dispensed directly from $1 \mathrm{mM}$ working stocks using a Liquid-handling Robot System (section 3.1.4).

\subsubsection{Controls for HTS}

Each plate in an HTS assay had a number of controls. The positive control included two standard anti-tubercular antibiotics, rifampicin and streptomycin at $20 \mu \mathrm{M}$, negative controls with no drugs and media controls with no cells (Figure 2.2). Since the chemical libraries were resuspended in DMSO, a DMSO control was also included in each plate. All controls were run in duplicate. 


\subsubsection{Liquid-handling Robot System}

HTS were performed by using a Liquid handler CyBio-96 ${ }^{\circledR}$. All plates were dispensed with high efficiency and good well-to-well accuracy in standard 96-wells plates with 80 compounds per plate, each compound at $20 \mu \mathrm{M}$ concentration.

\subsubsection{Objectives}

- To screen chemical libraries against the slow-growing species, $M$. bovis BCG and $M$. tuberculosis H37Ra.

- To identify and select potential growth inhibitors of M. tuberculosis.

- To validate inhibitors identified in HTS in an in vitro model of Tb, M. tuberculosis H37Ra. 


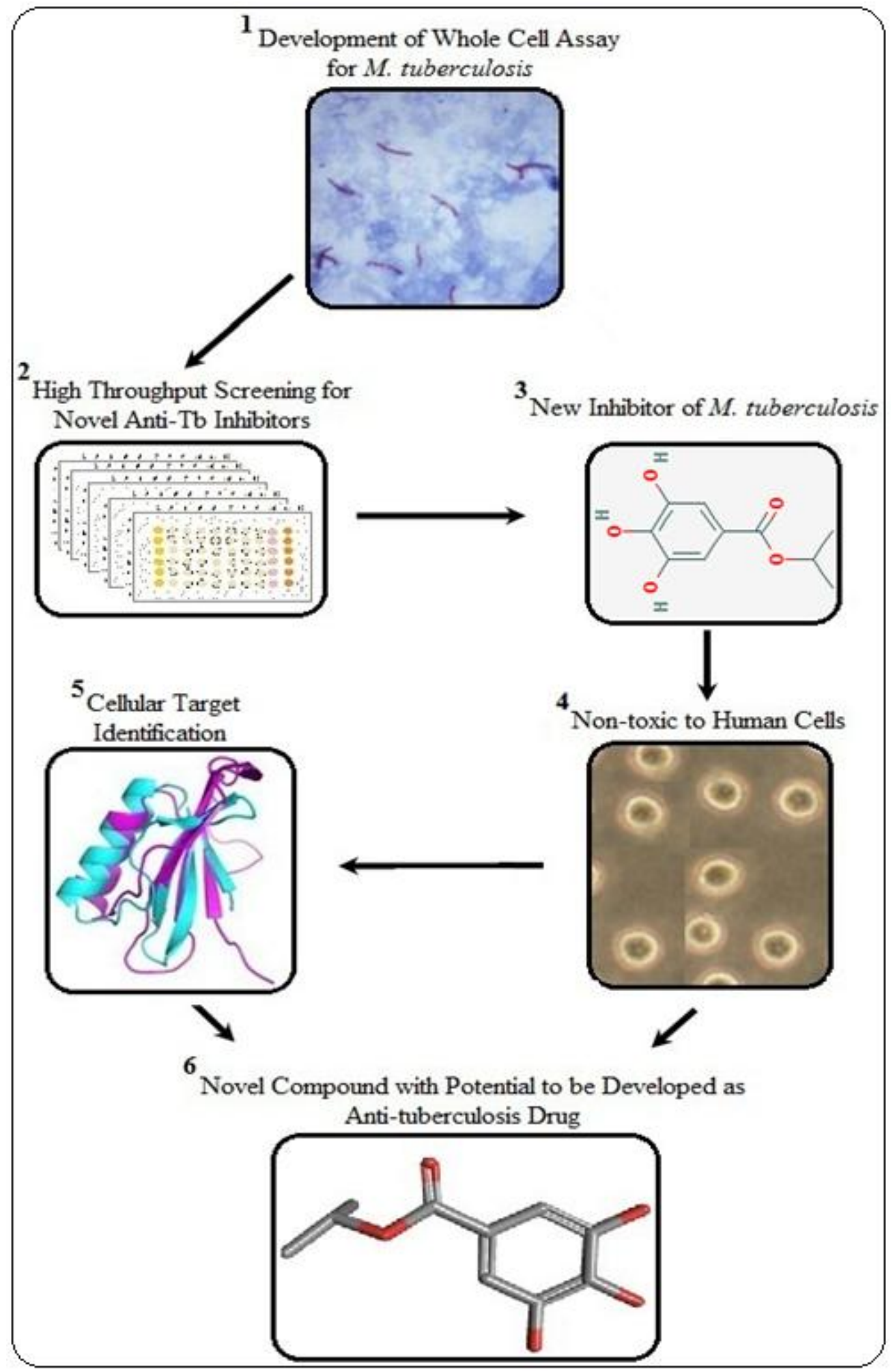

Figure 3.1: A schematic flow diagram of main research steps and objectives for this study (1) Develop a cell based assay to detect inhibitors of M. tuberculosis (2) Perform HTS of chemical libraries 3) Validate $M$. tuberculosis inhibitors using Tb model, M. tuberculosis H37Ra (4) Test for toxicity towards human cells (5) Characterize the possible drug target and (6) Finally select inhibitor(s) based on its therapeutic potential and 
unique mechanism of action, which can then be developed as a novel anti-Tb drug in collaboration with Global Alliance for Tb Drug Development.

\subsection{Development of a Biological Assay for High Throughput Screening}

\subsubsection{Growth Inhibition Assay}

A bacteriostatic assay was set up to determine growth inhibition of the slow-growing mycobacterial species, M. bovis BCG and M. tuberculosis H37Ra caused by the inhibitory activity of chemical compounds (section 3.2.3).

\subsubsection{Tb Models for HTS}

A slow-growing $\mathrm{Tb}$ vaccine strain was used as a surrogate species for screening of compound libraries (section 1.3.2.1). M. bovis BCG was transformed with an E. coli-BCG shuttle plasmid (pYL_gfp+kan ${ }^{\mathrm{r}}$ ) which has a green fluorescent protein (GFP) cloned from Aequorea victoria and a kanamycin resistance gene $\left(\operatorname{kan}^{\mathrm{r}}\right)$ downstream of the mycobacterial $h s p 70$ promoter. The gene encoding for GFP fluorescence is constitutively expressed by the $h s p 70$ promoter [277]. This genetically modified strain (Figure 3.2) was used as a screening model for HTS in this study (Table 2.1) and growth cultures were maintained as previously described [256]. The fluorescence expression was observed as described in section 2.7.2.1. 


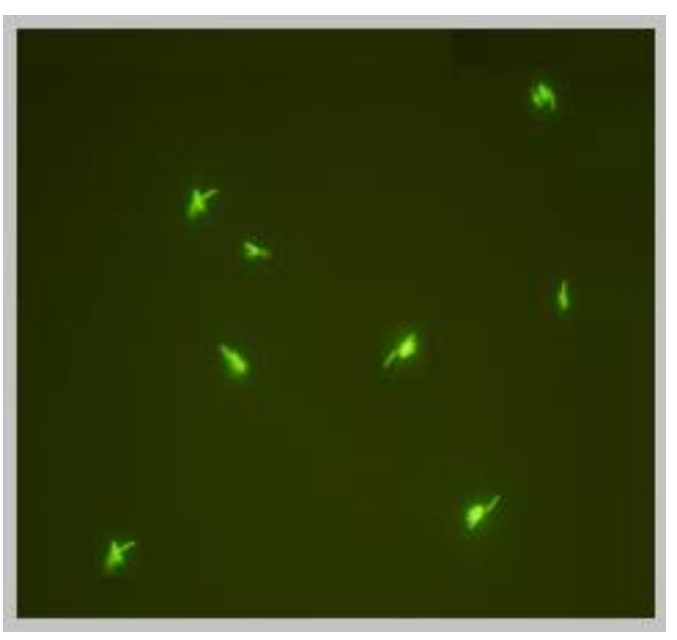

Figure 3.2: $M$. bovis BCG Pasteur 1173P2 fluorescently labelled with pYL_gfp.

\subsubsection{M. tuberculosis H37Ra}

Another slow-growing species, M. tuberculosis H37Ra that is closely related to $M$. tuberculosis $\mathrm{H} 37 \mathrm{Rv}$, was used in screening and for subsequent validation of mycobacterial inhibitors (sections 3.2.4). While both strains produce similar drug susceptibility data (section 4.1.1), $\mathrm{H} 37 \mathrm{Ra}$ is non-pathogenic in nature which makes it a preferable choice to be used as a surrogate for $\mathrm{H} 37 \mathrm{Rv}$ pathogenesis, in PC2 labs.

\subsubsection{M. smegmatis $\mathrm{mc}^{2} 155$}

M. smegmatis is a fast-growing mycobacterial species with a doubling time of 4 hours. It was employed for the purpose of comparing inhibitors across the different model organisms used in HTS to evaluate the sensitivity of each species to detect anti-Tb compounds (section 4.1.1).

\subsubsection{Available Reporter Systems for Mycobacterial Assays}

Growth is an effective indication of cell replication but a simple means of measuring optical density is not reliable in determining cell viability because mycobacteria tend to clump in 
broth cultures. Therefore, several techniques have been developed over the past decade to detect the viability of mycobacterial cells in HTS and growth inhibition assays. Examples include, a luciferase gene for bioluminescent signals [278], a gene encoding GFP harboured by a mycobacterial plasmid [279] or uptake of radio-labelled uracil by live cells [280]. Reporter systems have been widely used for several biological assays for viability and for monitoring cellular processes such as transcription. Like the Luciferase reporter, GFP has also been increasingly used in biological research with an efficient reporting ability in various biological models [281-282]. These reporter molecules, luciferase [283] and GFP [284], have the potential for drug screening in high-throughput with both reliability and cost effectiveness. However, unlike the luciferase gene-based assay, GFP does not require any exogenous substrates and/or cofactors [285-286]. Zafer et al. first reported the use of a reporter system in slow growing mycobacteria and the results from their study were based on a correlation between the fluorescence signal and CFUs [287]. Calculating CFU's is time consuming and can be labour intensive. Therefore, we used the correlation between $\mathrm{OD}_{600}$ values and the GFP signal. A close correlation between these two measurements ensured data reliability and accuracy. Table 3.1 presents the available fluorescence-based assays for drug screenings. 
Table 3.1: Reporter assays for high-throughput screening

\begin{tabular}{|c|c|c|c|}
\hline \multirow[t]{2}{*}{ Potential for HTS ${ }^{1}$} & \multicolumn{3}{|c|}{ Techniques } \\
\hline & $\mathrm{MABA}^{2}$ & Luciferase based assay & SFP based assay \\
\hline Sensitivity of the assay & Sensitive & Sensitive & Sensitive \\
\hline Substrates/co-factors & Dye required & Co-factors required & Not required \\
\hline \multicolumn{4}{|l|}{ Requirement } \\
\hline Reliability & Reliable & Highly reliable & Highly reliable \\
\hline Efficiency & Efficient & Efficient & Excellent \\
\hline Shaking requirements & Not required & Required & Required \\
\hline Reading device & Fluorometer & Luminometer & Fluorometer \\
\hline Expression analysis & MIC/FUs & RLUs & RFUs \\
\hline Fluorescence/CFUs & Yes & Yes & Yes \\
\hline \multicolumn{4}{|l|}{ Comparison } \\
\hline Effect of vector on & Not Required & No effect & No effect \\
\hline \multicolumn{4}{|l|}{ Drug susceptibility } \\
\hline Cost effectivity & Cost effective & Cost effective & Cost effective \\
\hline Assay reading $^{3}$ & Change in colour & Fluorescence & Fluorescence \\
\hline Use of mutant strains & No use & Yes & Yes \\
\hline Assay better than & BACTEC460 systems & Broth macro dilution assays & Luciferase assay \\
\hline
\end{tabular}

${ }^{1}$ Efficiency and capacity of various screening assays. ${ }^{2}$ Microplate-based Alamar Blue Assay. ${ }^{3}$ Assay reading time depends on optimum conditions and the type of strain used. GFP was exclusively selected for the determination of cell viability based on its efficiency [288]. RLUs, Relative Luminescence Units. RFUs, Relative Fluorescence Units.

The reason for comparing various available techniques is cost. The GFP reporter system is cost effective and does not require exogenous co-factors and/or substrates and is more 
convenient to use. Therefore, $M$. bovis BCG harbouring pYL_gfp plasmid was used for drug susceptibility testing in this study.

\subsubsection{Development and Optimization of a Fluorescence-based Assay}

All cell-based screening experiments were performed in 96-well plates with a total volume of $100 \mu \mathrm{L}$ per well as described (section 2.7.2) with appropriate controls in each assay plates (Figure 7.12). At the end point, plates were read for GFP fluorescence and $\mathrm{OD}_{600}$ (section 2.7.2.1.2)

\subsubsection{Growth Curves for M. tuberculosis H37Ra and M. bovis BCG}

The optimal inoculum and end point of the assay was determined by testing the variables in growth curves. Cultures were grown in 7H9 medium under standard growth conditions for mycobacteria as described (section 2.7.2.1). The $\mathrm{OD}_{600}$ and fluorescence of cultures were determined every day after the first 6 days of incubation for up to 14 days. The growth was also verified by CFU counts from these cultures. A consistent increase in optical density of cultures indicated that both the slow growing strains used in this study (M. tuberculosis H37Ra and M. bovis BCG) were growing for up to two weeks, after which cultures entered into stationary phase. There was no change in $\mathrm{OD}_{600}$ after two weeks of incubation, suggesting stationary phase had occurred (Figure 3.3). Therefore, assay reading time was set at day 14, before the cultures reached growth saturation. Cultures of different ages were tested for growth rates and to determine exactly when $M$. tuberculosis cells reach stationary phase. A noticeable difference was observed in the number of viable cells in logarithmically growing cultures versus stationary cultures. The CFU counts of an $M$. tuberculosis culture $\left(\mathrm{OD}_{600}\right.$ of $\left.\sim 1.0\right)$ was $1.54 \times 10^{5} \mathrm{CFUs} / \mathrm{mL}$ for log-phase cultures; whereas stationary cultures 
(more than four weeks old) gave a slightly lower number of viable cells i.e. $0.94 \times 10^{5}$ CFUs/mL. Figure 3.3 presents growth curves for both slow-growing mycobacterial species.

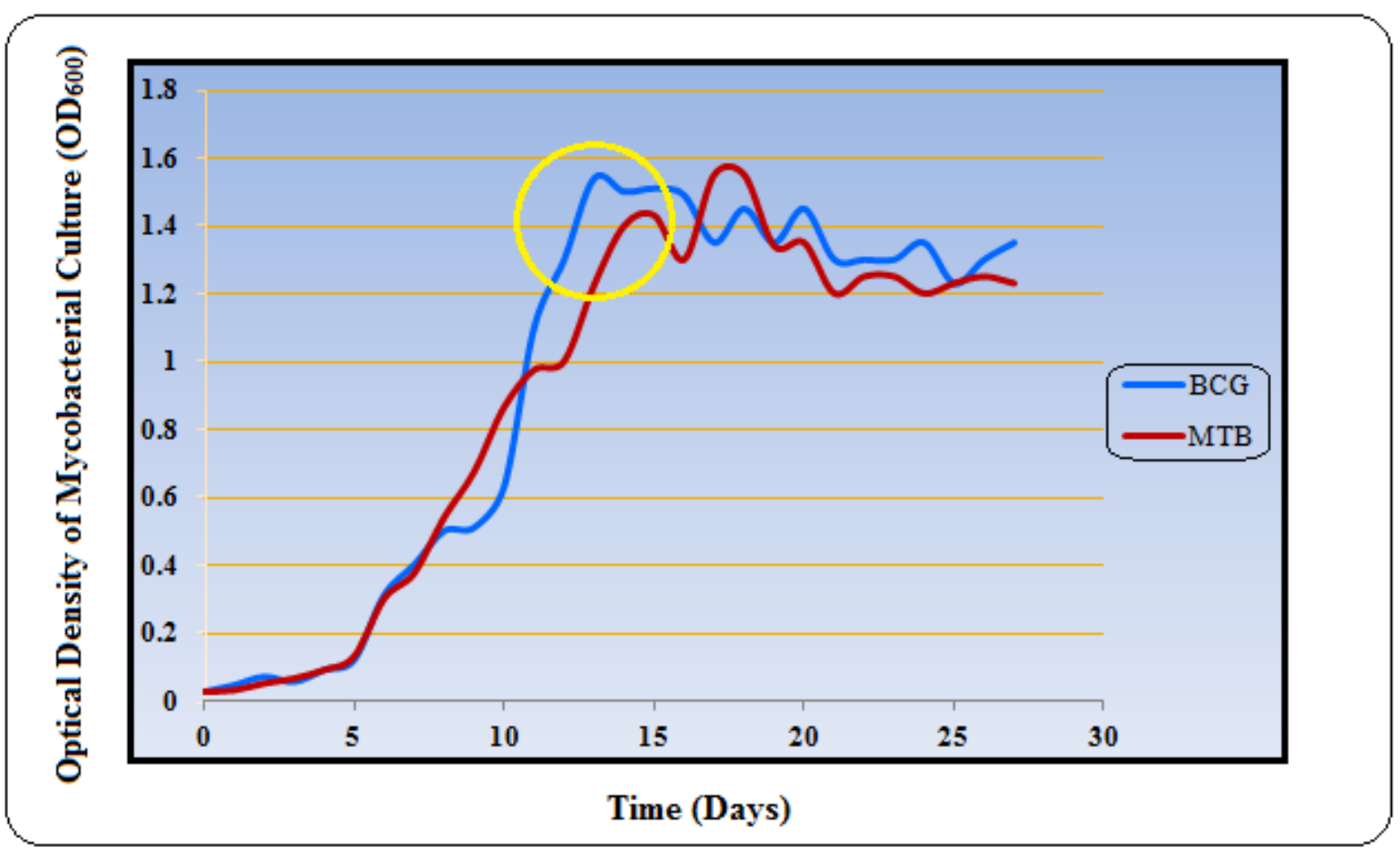

Figure 3.3: Time point growth curve for slow-growing mycobacteria. There was almost no change observed in $\mathrm{OD}_{600}$ readings post two weeks incubation indicating a stationary time point (circled) in M. bovis BCG and $M$. tuberculosis $\mathrm{H} 37 \mathrm{Ra}$ cultures; therefore, assay plates were read on day 14 of incubation for slow-growing mycobacteria. Both cell cultures were tested for $\mathrm{OD}_{600}$ every day and cells were mixed well to break their clumps and for their even distribution before readings. All $\mathrm{OD}_{600}$ values are the average of triplicate readings that were read each day from day zero to day 26.

\subsubsection{Reliability of a Bacteriostatic Assay}

A range of standard anti- $\mathrm{Tb}$ drugs were used in order to evaluate the reliability of the mycobacteriostatic assay. Published MIC values for standard anti-Tb drugs against $M$. tuberculosis $\mathrm{H} 37 \mathrm{Rv}$ were compared directly with the MICs values determined against $M$. 
bovis BCG in this study. Growth controls without any drug and sterility controls (medium only) were also included in each assay plates.

\subsubsection{Confirmation of the Assay Sensitivity}

To ensure assay sensitivity, the inhibitory effects of known anti-tubercular drugs were tested against $M$. bovis BCG. A close correlation of MIC values was observed among standard antitubercular drugs determined with $M$. bovis BCG to that of published values against $M$. tuberculosis H37Rv (Table 3.2). 
Table 3.2: Comparison of MIC values between M. bovis BCG and M. tuberculosis H37Rv

\begin{tabular}{lcccc}
\hline Anti-Tb Drug & \multicolumn{2}{c}{${ }^{1} \mathrm{H} 37 \mathrm{Rv}$} & & \multicolumn{2}{c}{ M. bovis $\mathrm{BCG}$} \\
\cline { 2 - 2 } & $\mathrm{MIC}(\mu \mathrm{M})$ & & $\mathrm{MIC}(\mu \mathrm{M})$ & $\mathrm{IC}_{50}(\mu \mathrm{M})$ \\
\hline Rifampicin & 0.48 & & 0.15 & 0.006 \\
Isoniazid & 0.183 & & 0.188 & 0.08 \\
${ }^{3}$ Pyrazinamide & 100 & & $>200$ & - \\
Ethambutol & 2.5 & & 2.8 & 1.9 \\
Streptomycin & 1.72 & & 1.25 & 0.6 \\
Capreomycin & 2.6 & & 1.64 & 0.36 \\
Ethionamide & 1.5 & 20 & 12.6 \\
D-Cycloserine & 245 & 265 & 205 \\
Ciprofloxacin & 1.5 & 1.55 & 0.98 \\
\hline
\end{tabular}

${ }^{1}$ Reported MICs for M. tuberculosis H37Rv adapted from the Handbook of Anti-Tuberculosis Agents [178]. ${ }^{2}$ Comparison of MICs against M. bovis BCG and M. tuberculosis H37Rv strains to evaluate the sensitivity of the growth inhibition assay used in this study. ${ }^{3}$ While pyrazinamide is active against $M$. tuberculosis, bovine mycobacterial strains are naturally resistant to pyrazinamide due to a point mutation in pnc $\mathrm{A}$ and therefore, no activity was observed at highest concentration tested $(200 \mu \mathrm{M})$. The comparison of MIC values in M. bovis BCG with M. tuberculosis H37Rv suggests that there was no effect of having a plasmid in the working M. bovis BCG model used for screening. (-) not calculated. MIC, minimum inhibitory concentration. $\mathrm{IC}_{50}$ values were calculated by Sigma Plot version 11.0 (Dose-response curves for standard drugs against M. bovis BCG are presented in figure 7.5).

\subsubsection{Assay Validation - Dose Response Assays}

M. tuberculosis $\mathrm{H} 37 \mathrm{Ra}$, an unlabelled avirulent strain of $\mathrm{Tb}$ was used for the validation of the anti-tubercular compounds identified and to determine their in vitro efficacy. Unlike the screening model, M. bovis BCG, M. tuberculosis H37Ra does not have the GFP reporter 
system or the antibiotic resistance cassette. Therefore, results for validation assays performed with M. tuberculosis H37Ra are based on optical density $\left(\mathrm{OD}_{600}\right)$.

\subsubsection{Effect of DMSO on M. bovis BCG Growth}

Since chemical libraries (and individual compounds tested for validation) were dissolved in DMSO and $2 \mu \mathrm{L}$ (of $1 \mathrm{mM}$ library working stocks) of each compound was dispensed making $2 \%$ final concentrations of DMSO in each well, it was deemed necessary to determine if DMSO itself had any inhibitory effect over M. bovis BCG growth. Figure 3.4 shows $M$. bovis BCG growth with up to 5\% DMSO.

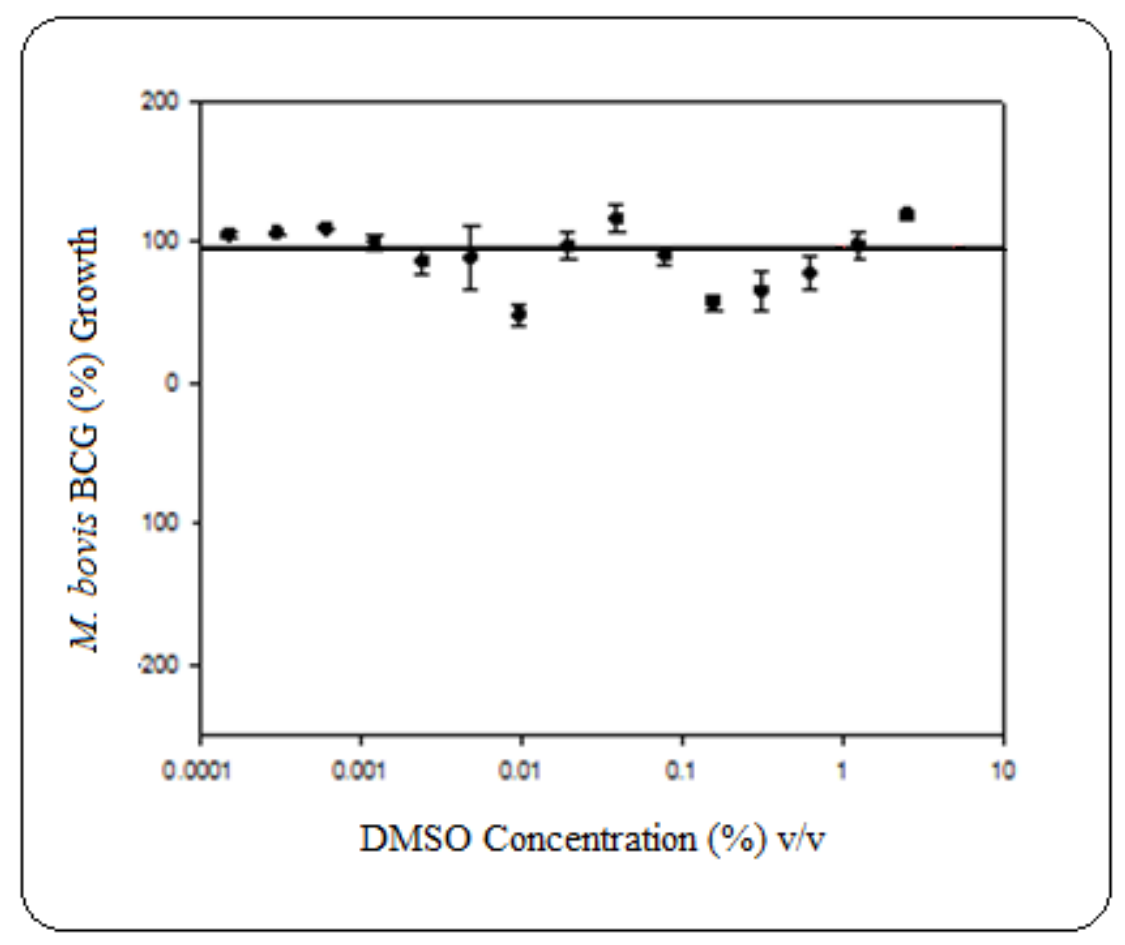

Figure 3.4: Percentage M. bovis BCG growth in DMSO. There was no inhibition of M. bovis BCG growth observed in a bacteriostatic assay with up to $5 \%$ DMSO. 


\subsection{Results}

\subsubsection{Compound Libraries for HTS}

Various chemical libraries consisting of over 5,000 distinct compounds were obtained as described (section 2.6) and were screened in high-throughput against M. bovis BCG (Figure 2.1).

\subsubsection{LOPAC ${ }^{1280 T M}$}

LOPAC was prepared in $250 \mu \mathrm{L}$ DMSO in $10 \mathrm{mM}$ stocks in 96-well plates with one compound per well. The library has 16 plates in total consisting of 80 individual compounds per plate. It has a wide range of compounds with antibacterial activities and known drug targets e.g. DNA gyrase inhibitors, cell wall and various cellular enzyme inhibitors [289]. LOPAC itself served mainly as an assay validation library; a preliminary screen was run against $M$. bovis BCG to identify known M. tuberculosis inhibitors in the library (Table 3.3). Figure 3.5 presents the distribution and class of pharmacologically active compounds in the LOPAC library with their wide range of biological targets. 


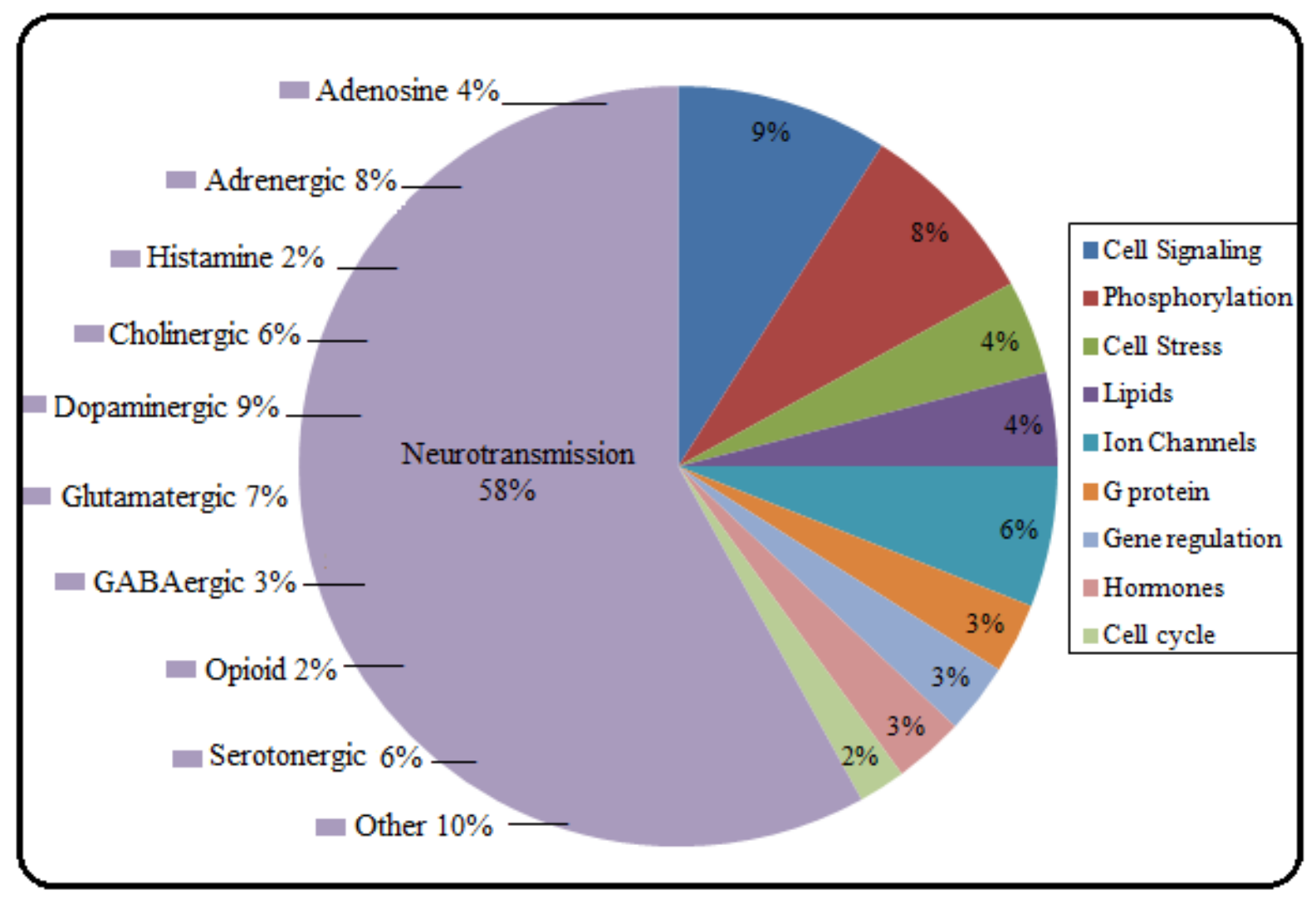

Figure 3.5: Distribution of compounds and range of drug targets in the LOPAC library. LOPAC has been designed to have a range of biological targets across bacterial, viral and fungal species [289].

\subsection{Scatter Plot for the LOPAC against M. bovis BCG}

An average of the triplicate values for each compound in a test plate was calculated, and the percentage $\mathrm{OD}_{600}$ and GFP fluorescence values were plotted together in a scatter plot to show hits, non-hits and values for a range of controls for HTS. Table 3.3 presents the positive antimycobacterial hits identified from LOPAC screen including their biological activity and the percentage inhibition they caused whereas, figure 3.6 shows the distribution of hits and controls used in LOPAC screen. 


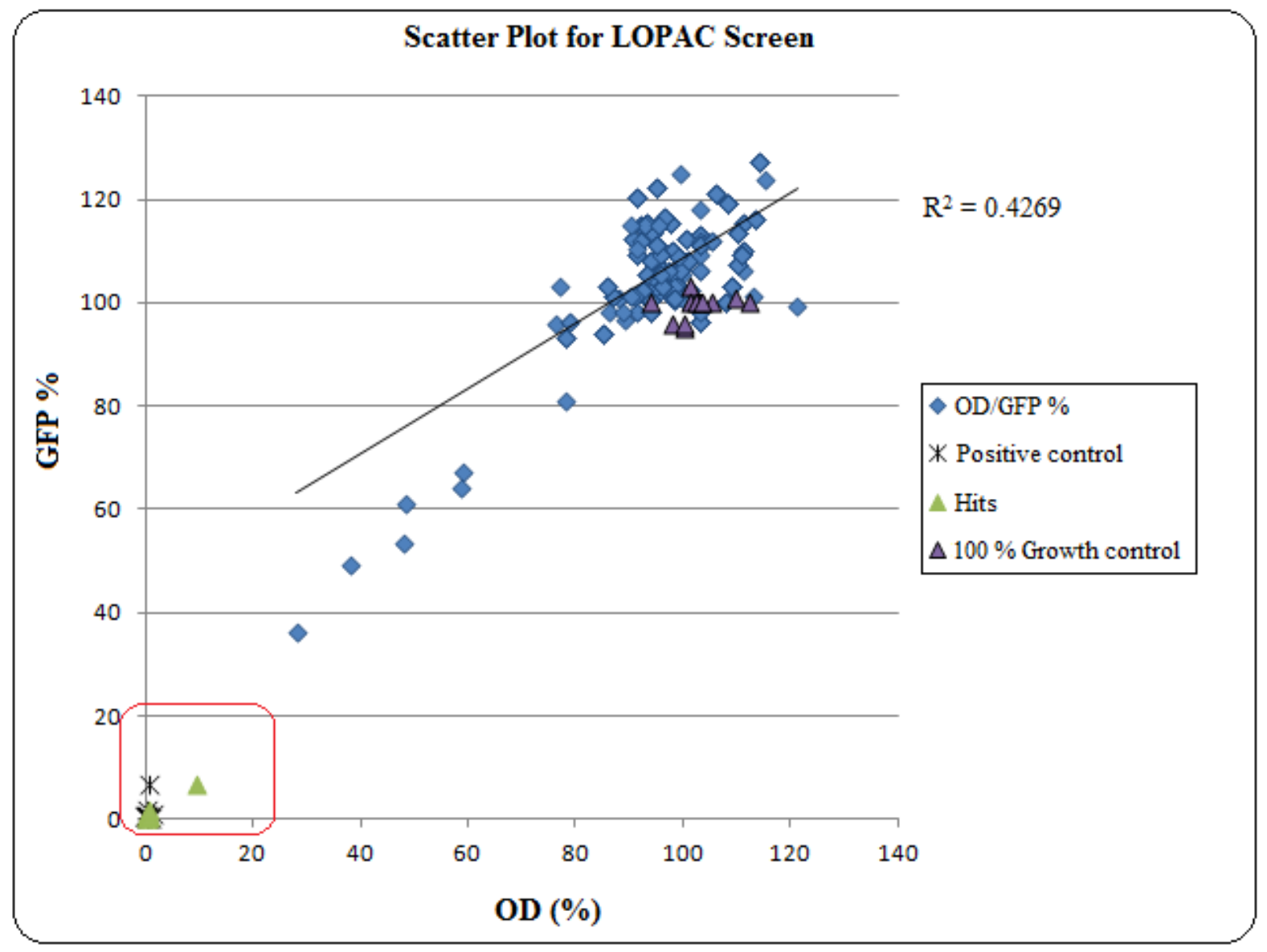

Figure 3.6: Scatter plot for LOPAC screen against $\boldsymbol{M}$. bovis BCG. $\mathrm{OD}_{600}(\%)$ values were plotted on the $x$ axis and GFP fluorescence (\%) values on the $y$-axis. The red square shows the $25 \%$ cut off for the identification of a positive hit i.e. at least $75 \%$ growth inhibition and also shows a positive control (rifampicin) that was included in each plate. Readings for both GFP fluorescence and $\mathrm{OD}_{600}$ show a good correlation $\left(\mathrm{R}^{2}\right.$ value 0.4269). The Z-factor was calculated to ensure fidelity of the screen, $\mathrm{Z}=0.91$ and 0.92 for both positive controls rifampicin and streptomycin, respectively.

In LOPAC scatter plot, while many inhibitors displayed only $50 \%$ inhibition of $M$. bovis BCG growth, some compounds had high fluorescence signal and their corresponding $\mathrm{OD}_{600}$ values showed relatively less or no inhibition compared to $100 \%$ growth control recorded in this study. Since these inhibitors did not fit our compound selection criteria (section 3.3.1.1.4), they were not considered for further characterization. However, compounds which inhibited mycobacterial growth by $>75 \%(n=24)$ from LOPAC screen are presented in table 3.3. Hits 
that appeared active twice during a triplicate screen were validated against $M$. tuberculosis in a dose-response assay designed to achieve measureable inhibitory concentrations. 
Table 3.3: Hits identified from LOPAC screen against $M$. bovis BCG and $M$. tuberculosis

\begin{tabular}{|c|c|c|c|c|}
\hline \multirow{2}{*}{$\begin{array}{l}\text { Compound } \\
\text { APDC }\end{array}$} & \multirow{2}{*}{$\frac{\mathrm{BCG}}{+}$} & \multirow{2}{*}{$\begin{array}{l}M t b \\
+\end{array}$} & \%Inhibition & \multirow{2}{*}{$\frac{\text { Class/Action }}{\text { ed anti-tubercular compound [290] }}$} \\
\hline & & & 99 & \\
\hline Calmidazolium chloride & + & + & 99 & Inhibitor of $\mathrm{Ca}_{2}+$ ATPase \\
\hline Clotrimazole & - & + & 80 & Inhibitor of $\mathrm{Ca}_{2}+$-activated $\mathrm{K}+$ channels \\
\hline Cephapirin sodium & + & + & 99 & Cell wall inhibitor [291] \\
\hline Calcimycin & + & + & $>75$ & $\mathrm{Ca}_{2}+$ ionophore [292] \\
\hline Diphenyleneiodonium $\mathrm{Cl}^{-}$ & + & + & 95 & Nitric oxide synthase inhibitor [293] \\
\hline Dequalinium chloride & + & + & $>75$ & Anti-tubercular[294] \\
\hline Doxycycline hydrochloride & + & + & 99 & Antibiotic \\
\hline Demeclocycline $\mathrm{HCl}$ & + & + & 99 & Antibiotic \\
\hline Fusidic acid sodium & + & + & 95 & Protein synthesis inhibition in prokaryotes \\
\hline Ellipticine & + & + & 95 & Anti-tubercular, TAACF [295] \\
\hline 5-Fluorouracil & + & - & 75 & Thymidylate synthetase inhibitor \\
\hline Idarubicin & + & - & 85 & Anti-cancer (PubChem database) \\
\hline Lomefloxacin $\mathrm{HCl}$ & + & + & 99 & Antibiotic/DNA gyrase inhibitor \\
\hline Minocycline $\mathrm{HCl}$ & + & + & 90 & Protease inhibitor \\
\hline Methoctramine tetra $\mathrm{HCl}$ & + & + & 90 & Antagonist \\
\hline Nialamide & + & + & 85 & Monoamine oxidase inhibitor \\
\hline Niclosamide & + & + & 90 & Antibiotic/protonophore [295] \\
\hline Pentamidine isethionate & + & - & 75 & Known antimicrobial [296] \\
\hline Resveratrol & + & - & 85 & anti-M.smegmatis [297] \\
\hline Seglitide & - & + & 75 & sst2 somatostatin receptor agonist \\
\hline 1, 10-Phenanthroline & + & + & 95 & Metalloprotease inhibitor \\
\hline Tetraethylthiuram disulfide & + & + & 99 & Alcohol dehydrogenase inhibitor \\
\hline Vancomycin hydrochloride & + & + & 99 & Antibiotic (known) \\
\hline
\end{tabular}

Compounds were further selected for validation in M. tuberculosis if they were found active at least twice during a triplicate screen with no previously reported anti-mycobacterial activity. A (+) sign indicates positive hit in HTS against M. bovis BCG and was also validated in M. tuberculosis H37Ra. Whereas, (-) indicates no 
hit in that species. APDC, Ammonium Pyrrolinedithiocarbamate. TAACF, Tuberculosis Antimicrobial Acquisition and Coordinating Facility.

\subsection{Chromogenecity of Compounds}

Coloured compounds appeared to interfere with the GFP signal and the $\mathrm{OD}_{600}$ measurements. To overcome this, $100 \mu \mathrm{L}$ of medium without cells was added to separate wells with these compounds and the readings for each well were subtracted from the growth readings of the test compounds to correct for the high background. 
Table 3.4: Validated hits against $M$. tuberculosis from the LOPAC library

\begin{tabular}{lccccc}
\hline Compound & Structure & $(\mathrm{MICs} \mu \mathrm{M})$ in BCG & $M t b$ & Molecular Weight & Formula \\
\hline TETD & 6.25 & 3.125 & $296.52 \mathrm{~g} / \mathrm{mol}$ & $\mathrm{C}_{10} \mathrm{H}_{20} \mathrm{~N}_{2} \mathrm{~S}_{4}$
\end{tabular}<smiles>CCN(CC)C(=S)SSC(=S)N(CC)CC</smiles>

APDC

1.56

0.781

164.28

$\mathrm{C}_{5} \mathrm{H}_{9} \mathrm{NS}_{2} \cdot \mathrm{NH}_{3}$<smiles>NC(=S)N1CCCC1</smiles>

2-CA

12.5

12.5

301.69

$\mathrm{C}_{10} \mathrm{H}_{12} \mathrm{CIN}_{5} \mathrm{O}_{4}$<smiles>Nc1nc(Cl)nc2c1ncn2C1OC(CO)[C@@H](O)C1O</smiles>

FAS

3.125

1.56

538.71

$\mathrm{C}_{31} \mathrm{H}_{47} \mathrm{NaO}_{6}$

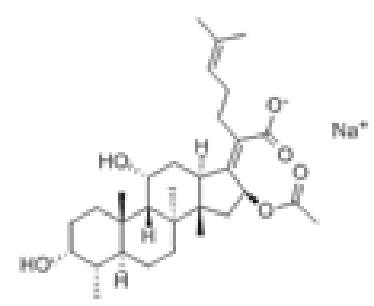

WB-64

12.5

12.5

808.66

$\mathrm{Br}_{2} \mathrm{C}_{40} \mathrm{H}_{48} \mathrm{~N}_{4} \mathrm{O}_{4}$

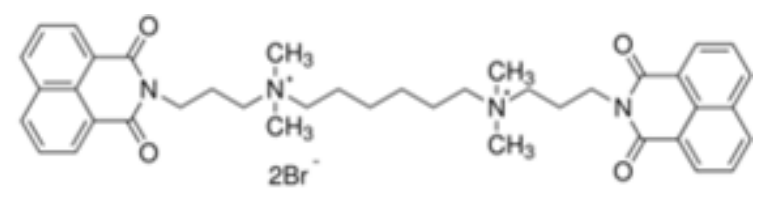

Compounds were validated in M. tuberculosis H37Ra. TETD, Tetraethylthiuram disulfide. APDC, Ammonium Pyrrolinedithiocarbamate. 2-CA, 2-Chloroadenosine. FAS, Fusidic acid sodium. Compound images were retrieved from [289]. 


\subsection{Brief Description of Validated LOPAC Hits and their Biological Activity}

Except for WB-64, hits listed in table 3.4 were $M$. bovis BCG and Mtb-specific, which did not appear in screens with $M$. smegmatis (Chapter 4). A brief description of their biological activity and any anti-mycobacterial activity is noted below.

\section{A. Tetraethylthiuram Disulfide (TETD)}

TETD, also known as Disulfiram, is a low molecular weight compound (296.52 g/mol) that inhibits the enzyme alcohol dehydrogenase. Disulfiram has been considered to be a safe drug and was previously used for the management of alcohol abuse and intoxication as well as being used as a soil treatment agent in the agricultural sector [298-300]. It has also been used for the treatment of $\mathrm{Tb}$ [301].

\section{B. Ammonium Pyrrolinedithiocarbamate (APDC)}

APDC is a low molecular weight compound $(164.28 \mathrm{~g} / \mathrm{mol})$ and is non-toxic. It bears anti-corrosive properties and has been used as a metal chelator [302-303]. APDC has previously been found to be active against the slow-growing mycobacterial species with an MIC against M. tuberculosis H37Rv of $0.791 \mu \mathrm{M}$ [290], in this study the MIC was $0.781 \mu \mathrm{M}$ against $M$. tuberculosis H37Ra (Table 3.4). Inhibition of $M$. tuberculosis growth may be associated with its metal chelating activity. Pyrrolinedithiocarbamate compounds also seem to have anti-viral properties in vitro [304].

\section{Fusidic Acid Sodium}

Fusidic acid was validated against M. tuberculosis H37Ra with an MIC value of 1.56 $\mu \mathrm{M}$. It has previously been reported to be anti-mycobacterial by Hoffner et al. (MIC 
range for $M$. tuberculosis $=0.032-0.064 \mu \mathrm{g} / \mathrm{mL}$ or $0.06-0.2 \mu \mathrm{M})$ and was found synergistically active with ethambutol in combination therapy [305]. Fusidic acids are known to interfere with protein synthesis of prokaryotes and are highly active against M. leprae infections with an $\mathrm{IC}_{50}$ of $0.3 \mu \mathrm{M}$ [306].

\section{2-Chloroadenosine}

This compound has been used in biological research for the modification of nucleoside and nucleotides. It has been shown by Mary et al. to be a metabolically stable analogue of adenosine, which acts as a selective adenosine receptor agonist. It also possesses anti-mycobacterial activity with higher inhibitory concentrations ranging from 3.0-33.3 $\mu \mathrm{M}$ [307]. While good anti-mycobacterial activity of 2Chloroadenosine was observed in this study (MIC $12.5 \mu \mathrm{M}$ against M. tuberculosis), it displays cytotoxicity to mammalian cells by inducing cell apoptosis [308].

\section{E. WB-64}

WB-64 [N,N,N',N'-tetramethyl-bis[(1,8-naphthylimid-9-yl)propyl]-N,N'-hexane-1,6diyl-bis(ammonium bromide)] is a white coloured and water soluble compound. It was one among many anti-mycobacterial hits against from the LOPAC screen and was detected with M. smegmatis with an MIC value of $50 \mu \mathrm{M}$ [252]. The present study identified it as being active against $M$. bovis BCG in HTS, and this activity was also validated in $M$. tuberculosis $\mathrm{H} 37 \mathrm{Ra}$ with an MIC of $12.5 \mu \mathrm{M}$ (Table 3.4). However, it has recently been identified as an inhibitor of human apurinic endonuclease [309].

LOPAC hits were primarily used to validate the HTS procedure used in this study and to evaluate the suitability of dose-response assays for the validation of compounds. However, 
there have been many compounds identified from both the Spectrum Collection and the NIH libraries that may hold potential for further development in the drug discovery process (section 3.3.1.3).

\subsection{Selection Criteria for Novel Compounds Identified Through HTS}

Compounds identified from HTS were only considered appropriate for further characterization if they fulfilled following pre-set selection criteria.

\section{A. Experimental Approach}

Compounds identified in the HTS have yielded $>75 \%$ anti-mycobacterial activity in triplicate screens (tested at $20 \mu \mathrm{M}$ ).

Appeared active when re-tested at a higher concentration $(100 \mu \mathrm{M})$ against $M$. bovis $\mathrm{BCG}$ in a dose-response assay.

$>$ Compounds are also validated against M. tuberculosis $\mathrm{H} 37 \mathrm{Ra}$ and have low inhibitory concentrations.

Compounds that retain good in vitro activity against $M$. tuberculosis H37Ra were put through an extensive literature search to confirm if a hit has potential to be further characterized.

\section{B. Literature-based Approach}

Literature mining was performed to find any previously reported activity against M. tuberculosis for the inhibitors identified.

Databases such as PubChem, Toxnet, Chemblink, DrugBank, TAACF and journal articles were accessed as a primary source of information on inhibitors identified in this study. 
After novelty check based on previously published data, compounds were further assessed on the basis of their toxicity profiles. New mycobacterial inhibitors were excluded, if those compounds were previously found toxic towards mammalian cells (e.g. anti-mycobacterial hits from NIH library thaspine, mitxantrone and nogalamycin), it was excluded from further study.

If compounds were known to target human enzymes, they were excluded as they would not make good anti-microbials.

A compound found to have any promiscuous activity or identified as an interference compound (if a compound has been identified in previous multiple active screens) was also not considered as a positive hit for M. tuberculosis and was left out of the hit list. Novel inhibitors are being sought in the present study must not have biological targets in humans and ideally should have had no previous active screens.

\subsubsection{Spectrum Collection}

A set of 2,000 compounds known as the Spectrum Collection was purchased from Microsource Discovery Inc. Each compound had a minimum of 95\% purity in the Spectrum library.

\subsection{Collection of compounds}

Spectrum library consisted of compounds containing drug-like properties from a range of resources. The majority of compounds (50\% of the library collection) were drug-like components. Thirty percent of the compounds $(n=580)$ were pure natural extracts and the rest of the compounds were non-enzyme inhibitors that might have biological activity ( $20 \%$ of the library collection). The distribution and type of Spectrum compounds are available from their website www.msdiscovery.com/spectrum.html. There was also an overlap of compounds, 
approximately $12 \%$ with the LOPAC including some known anti-bacterials or drugs with known mechanism of action. These compounds served as embedded controls for HTS to determine the reliability of the screens as well as to cross verify hits between libraries.

\subsection{Spectrum Screen against $M$. bovis BCG}

A triplicate screen (three plates with three wells per plate per compound) was performed twice (two separate screens, a total of 6 plates, 18 wells) to identify potential growth inhibitors from the Spectrum Collection, in which a close correlation both in terms of number as well as types of hits was found (Figure 3.7). An average of approximately 62 hits was achieved with a hit rate of $3.1 \%$.

\section{Comparison of Hits Between Triplicate Spectrum Screens}

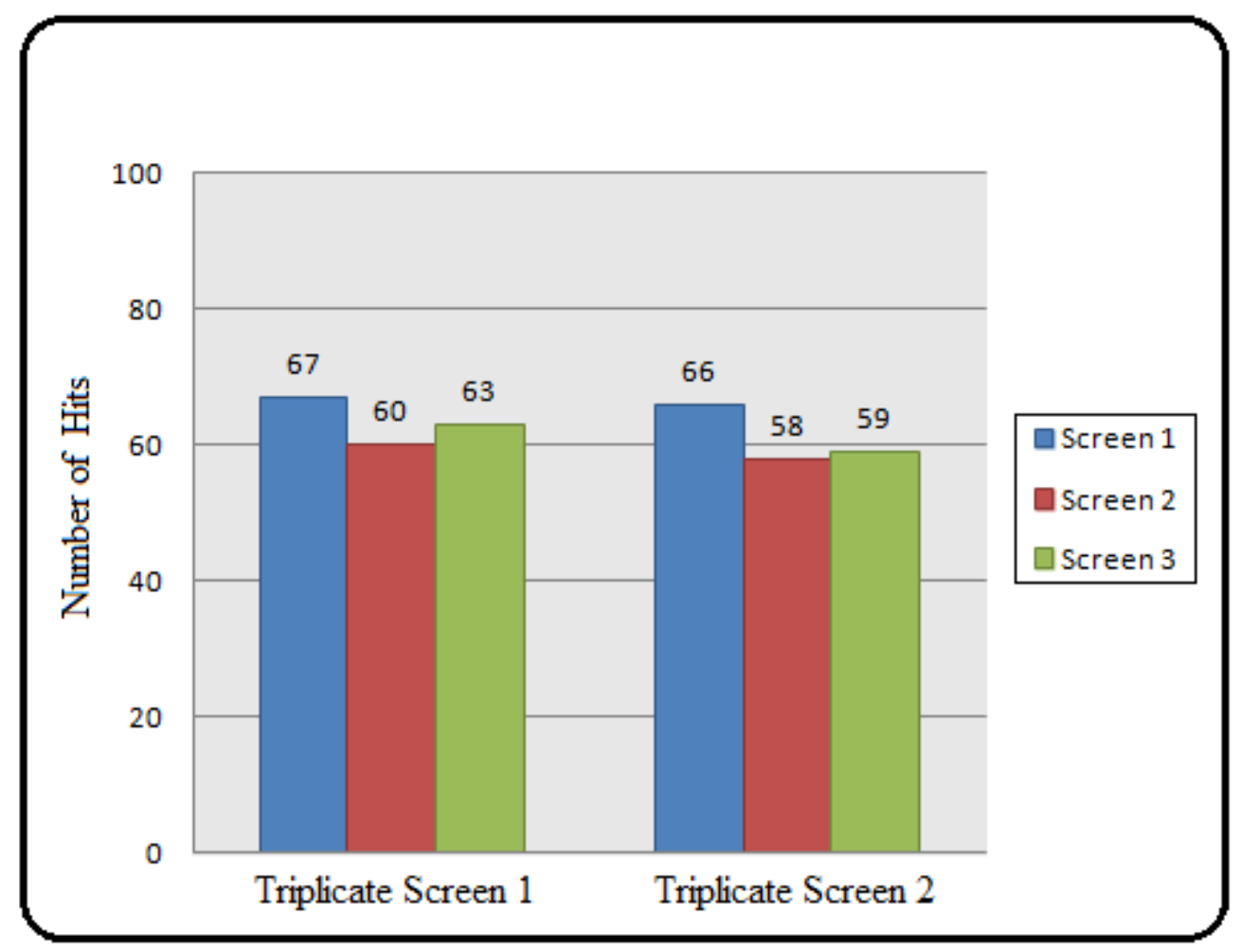

Figure 3.7: Number of hits identified in two independent triplicate Spectrum screens against $M$. bovis

BCG. Results from triplicate screens were compared to ensure data integrity and to examine if type of hits were consistent in each screen (see figure 3.9). 


\subsection{Scatter Plot for Spectrum Screen}

A scatter plot was made using the percentage $\mathrm{OD}_{600}$ and GFP fluorescence values to identify positive hits compared to positive controls (Figure 3.8).

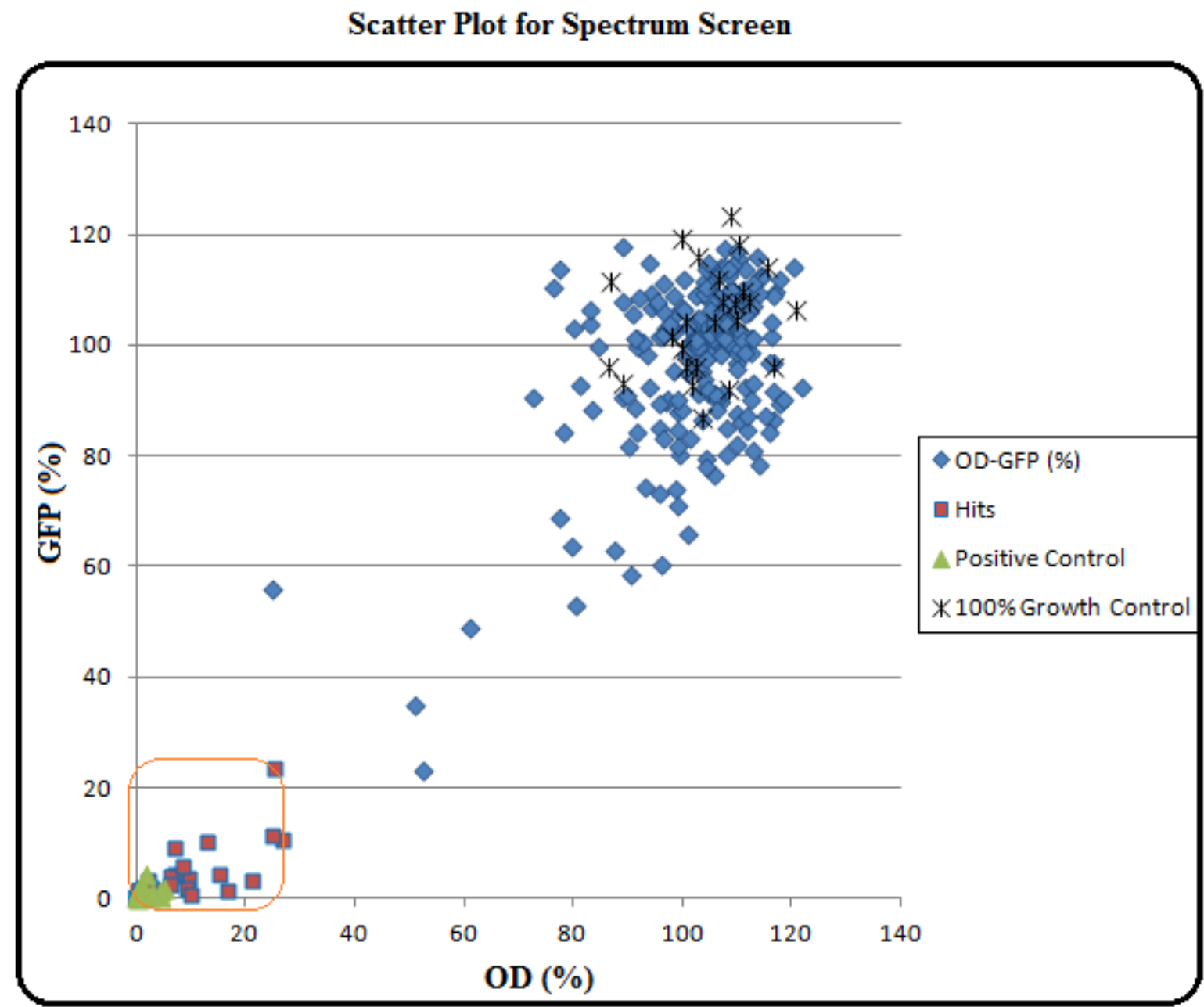

Figure 3.8: Scatter plot for Spectrum screen. The red-square dots within the cut off value $(>75 \%$ growth inhibition, orange square) were identified as positive hits compared to the positive control, rifampicin, included in each plate. 
Table 3.5: Validated hits against $M$. tuberculosis from Spectrum library

\begin{tabular}{|c|c|c|c|c|}
\hline Compound & BCG & $M t b$ & \%Inhibition & Activity \\
\hline Aklavine $\mathrm{HCl}$ & + & + & 99 & Anti-malarial [310] \\
\hline Cloxyquin & + & + & 99 & Anti-tubercular [311] \\
\hline Disulfiram• & + & + & 99 & Anti-malaria [312] \\
\hline Ketoconazole & + & + & $>75$ & Anti-M. tuberculosis [295] \\
\hline Mercaptopurine & + & + & $>75$ & Mycobacterium paratuberculosis [313] \\
\hline Clotrimazol & + & + & 99 & Anti-tubercular [295] \\
\hline Pasiniazid & + & + & 99 & Anti-tubercular \\
\hline Cefdinir & + & + & 95 & Cephalosporin class, antibacterial [314] \\
\hline Econazole nitrate & + & + & 95 & Anti-tubercular [295] \\
\hline Alexidine $\mathrm{HCl}$ & + & + & 99 & Antibacterial/Anticancer activity [315] \\
\hline Orlistat & + & + & 90 & Mycolic acid biosynthesis inhibitor [316] \\
\hline Sappanone A* & + & + & 99 & Novel anti-Tb compound \\
\hline Dimethyl ether & & & & [This study] \\
\hline Acivicin & + & + & 99 & Inhibits tumour angiogenesis [317] \\
\hline Nigericin sodium & + & + & 95 & Disrupt membrane potential in $M t b$ [318] \\
\hline Carmofur & + & + & 95 & Anti-neoplastic agent [PubChem database] \\
\hline
\end{tabular}

Except aklavine $\mathrm{HCl}$, carmofur and clotrimazole, most compounds from Spectrum screens were detected with $M$. bovis BCG (and M. tuberculosis $\mathrm{H} 37 \mathrm{Ra}$ ) but not with M. smegmatis (data not shown). *Compounds with no previously published anti-mycobacterial activity. $(\bullet)$ Compound also appeared as a hit from the LOPAC library (Table 3.4) and was also active against the eukaryote parasite P. falciparum and was previously reported as an anti-tuberluar compound. 


\subsection{Description of Spectrum Hits with Anti-mycobacterial Activity}

While some compounds from the Spectrum libray are known to have antibiotic properties, many anti-mycobacterial hits identified in this study had been noted to have undetermined biological actitivity, (biological information of compounds was retrieved from MicroSource Discovery Inc). Compounds detected as positive hits in screens were not further pursued due to known toxicity or unfavourable therapeutic potential (section 3.3.1.1.4). However, compounds with no published reports of any specific anti-mycobacterial activity were considered novel such as sappanone A dimethyl ether, which may have good potential to be further characterized and developed into a drug (section 3.4). Some inhibitors have previously been reported to inhibit Plasmodium falciparum (P. falciparum) replication in vitro e.g. aklavine $\mathrm{HCl}$ and mitoxantrone [310]. These compounds also inhibited mycobacterial growth in the present study (Table 3.5), suggesting that these could be further investigated for mutual inhibitory activity against parasitic and bacterial pathogens (section 6.3.3). 
Comparison of Spectrum Hits, BCG Vs Mtb

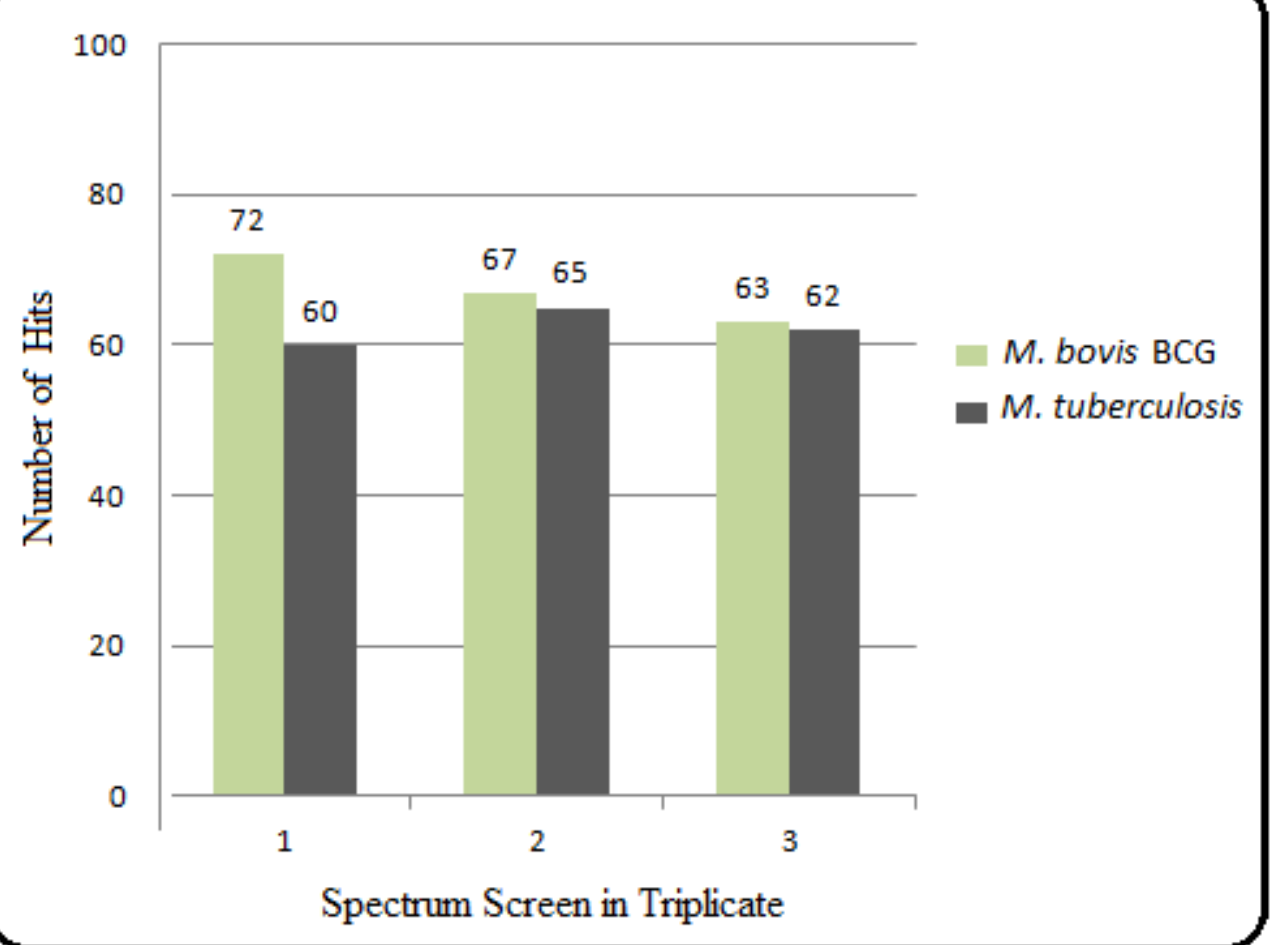

Figure 3.9: Comparison of hits from a triplicate Spectrum screen performed against M. bovis BCG and $M$. tuberculosis H37Ra. Hits appeared to be consistent with slow-growing mycobacterial species among all screens (see Table 3.5 for validated hits from the spectrum library and Figure 3.17 for common hits among all screens).

\subsubsection{NIH Library}

The NIH library has a wide range of structurally-diverse compounds with a range of biological activities. Plate-to-plate information and information on individual compounds were retrieved from the NIH website for Developmental Therapeutics Program (www.dtp.nci.nih.gov). The NIH library was divided into two portions.

1. NIH Diversity Set constituting 1990 compounds and

2. A mini library of NIH Natural Products containing 240 compounds

After the libraries were screened, information on positive hits was retrieved from the official website of PubChem database, available at www.ncbi.nlm.nih.gov/pccompound. 


\subsection{Scatter Plot for NIH Screen}

\section{Scatter Plot of HTS for NIH Chemical Library}

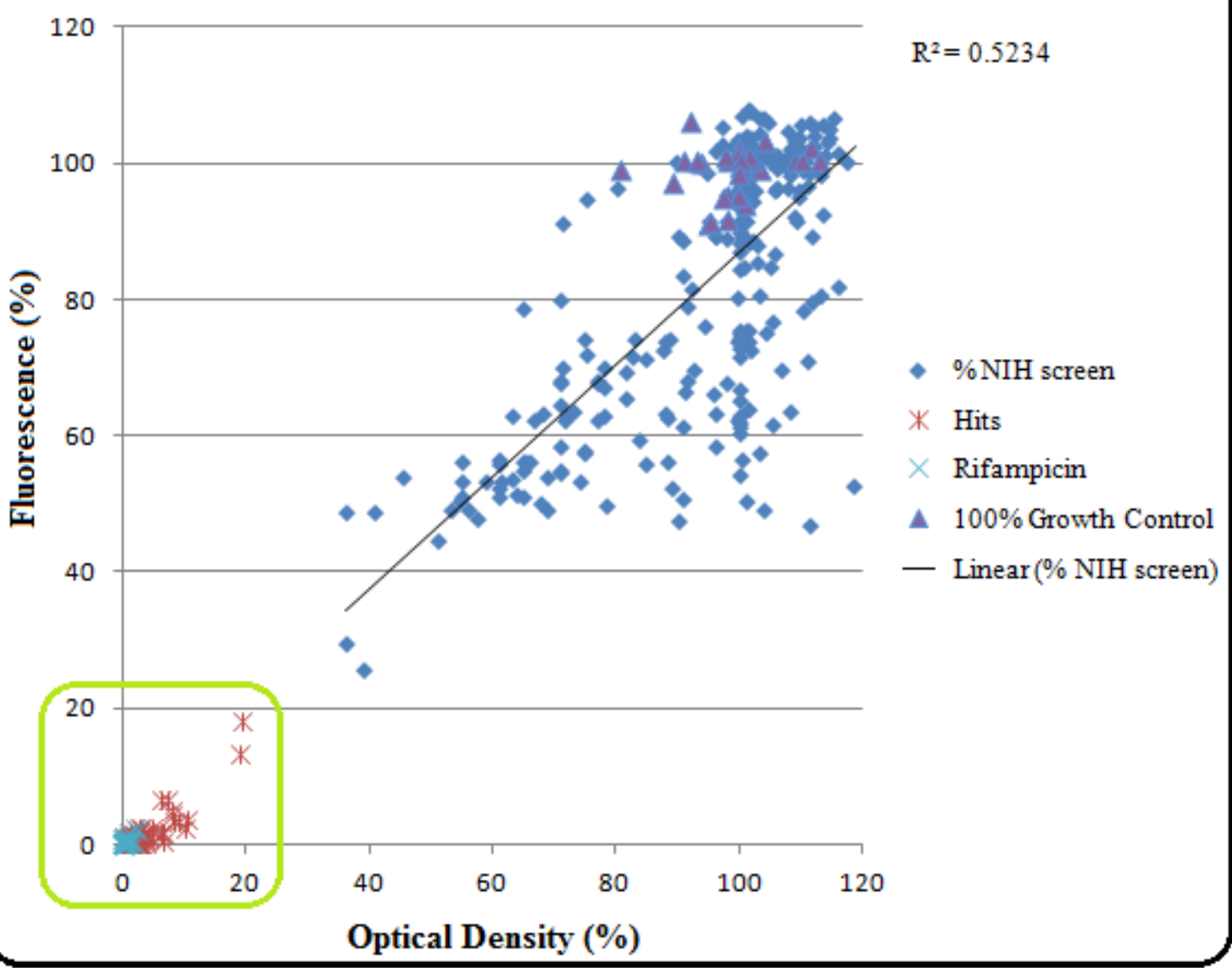

Figure 3.10: Scatter plot for NIH screen performed against $M$. bovis BCG. NIH library contained Diversity and Natural product sets constituting 2230 compounds. Hits with positive control (rifampicin) are shown within the cut-off value of $<25 \%$ growth (green square). Novel growth inhibitors selected from the NIH library are presented in table 3.6 .

\subsection{Potential Inhibitors of M. tuberculosis from NIH Library}

New hits identified from the NIH library screen that inhibited growth of $M$. bovis BCG and $M$. tuberculosis H37Ra have been listed in table 3.6. 


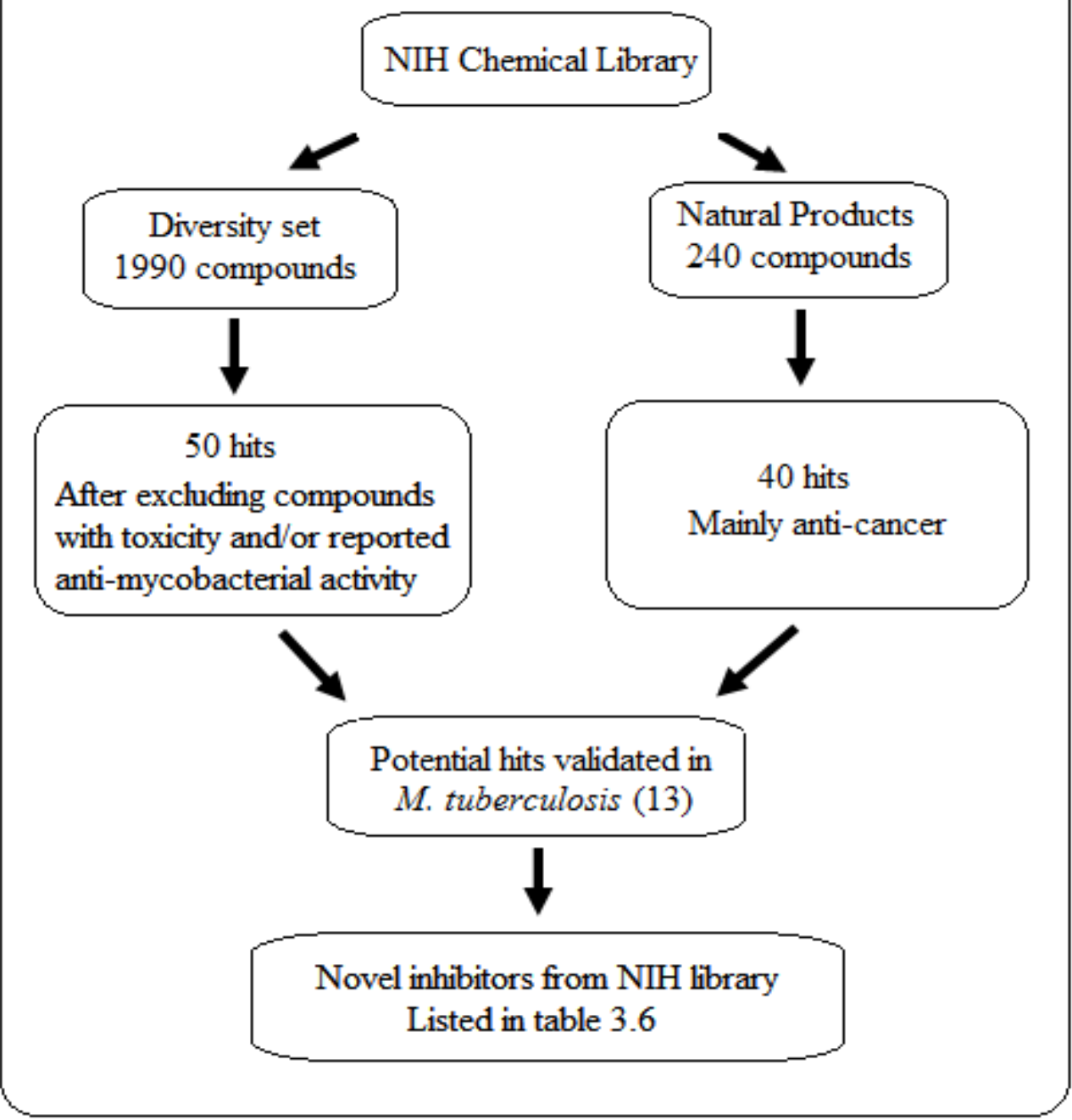

Figure 3.11: Flow chart for NIH chemical library screen. A total of 90 anti-mycobacterial hits were detected from the NIH screens against $M$. bovis BCG. All compounds were tested individually in dose-response assays against M. tuberculosis H37Ra. Inhibitors validated against M. tuberculosis $(\mathrm{n}=13)$ are listed in table 3.6, see also table 4.2 for active inhibitors of $M$. tuberculosis H37Ra identified from the NIH library ( $\mathrm{n}=28)$.

\subsection{Biological Evaluation of Hits from NIH Library}

After the hits have been identified as potential inhibitors of $M$. bovis BCG growth, they were evaluated for their biological activity from any previously published data. Biological activity and literature study indicated that many inhibitors such as NSC15381, NSC64111, NSC168466, NSC168467, NSC168468, NSC333003, NSC402959 and NSC603988 had no 
previously established anti-mycobacterial activity. However, a few of them were previously tested in other biological assays (non-Tb assays) in which they appeared as inactive (PubChem database). All compounds were then selected for further biological/toxicity evaluation and characterization. 
Table 3.6: Validated $M$. tuberculosis inhibitors from the NIH library

\begin{tabular}{|c|c|c|c|}
\hline \multirow[t]{2}{*}{ Compound } & \multicolumn{3}{|c|}{ Mycobacterial Species (MIC $\mu \mathrm{M}$ ) } \\
\hline & M. tuberculosis & M. bovis $\mathrm{BCG}$ & M. smegmatis \\
\hline NSC15381 & 0.781 & 0.39 & nd \\
\hline NSC16865 & 12.5 & 25 & 6.25 \\
\hline NSC62709 & 12.5 & 50 & 25 \\
\hline NSC61586 & 1.56 & 25 & 25 \\
\hline NSC64111 & 25 & 50 & 50 \\
\hline NSC145611 & 6.25 & 50 & 100 \\
\hline NSC145612 & 50 & $>100$ & $>100$ \\
\hline NSC168466 & 1.56 & $>100$ & $>100$ \\
\hline NSC168467 & 12.5 & $>100$ & $>100$ \\
\hline NSC168468 & 3.125 & $>100$ & $>100$ \\
\hline NSC333003 & 1.56 & $>100$ & $>100$ \\
\hline NSC402959 & 12.5 & 25 & $>100$ \\
\hline NSC603988 & 0.39 & 0.39 & $n d$ \\
\hline
\end{tabular}

Some compounds $(\bullet)$ did not exhibit inhibitory activity in dose-response assays against $M$. bovis BCG for up to maximum concentration tested $(100 \mu \mathrm{M})$ and only demonstrated activity against $M$. tuberculosis (section 3.4). Compounds that showed an MIC of at least $<50 \mu \mathrm{M}$ against $M$. tuberculosis are listed. $n d$, not done.

\subsection{Potential M. tuberculosis Inhibitor (NSC402959) and its Biological Activity}

NSC402959 was identified during HTS from NIH chemical library and has not been previously reported to have anti-mycobacterial effect. It has been found active in previous screens for an E. coli RNase HI (PubChem database). It was also tested against a number of cell lines in this study and its cytotoxicity appeared to be in an acceptable range with an $\mathrm{IC}_{50}$ for both HL-60 and J774 cell lines being >150 $\mu \mathrm{M}$ (Figure 4.13 and Figure 7.8, respectively). 
NSC402959 called attention towards the identification of a novel class of $M$. tuberculosis inhibitors (Chapter 5). Figure 3.12 shows bacteriostatic activity of NSC402959 against $M$. tuberculosis H37Ra.

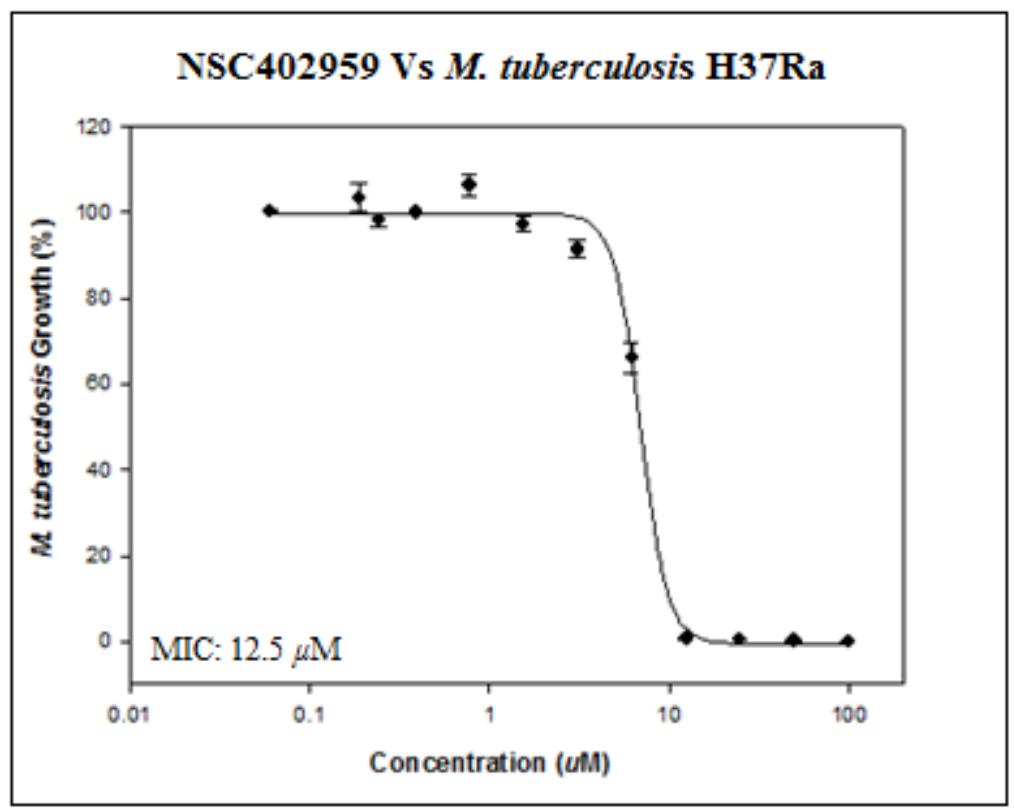

Figure 3.12: Dose-response of NSC402959 against M. tuberculosis H37Ra. MIC against the avirulent $M$. tuberculosis species (H37Ra) was $12.5 \mu \mathrm{M}$. NSC402959 also exhibited a similar level of inhibitory activity against virulent $\mathrm{Tb}$ model, M. tuberculosis $\mathrm{H} 37 \mathrm{Rv}$ (also see Figure 4.12 and Figure 7.7).

\subsection{Structural Analogues of NSC402959}

Two structurally related compounds NSC15381 and NSC603988 were imported individually from the NIH compound repository (section 4.5). Being structurally similar (Figure 4.14), they were expected to show anti-mycobacterial activity. In dose response assays, they were found to be highly active against $M$. tuberculosis $\mathrm{H} 37 \mathrm{Ra}$ with an MIC of $0.781 \mu \mathrm{M}$ and 0.39 $\mu \mathrm{M}$, respectively (Table 3.6). NSC15381 also exhibited inhibitory activity against clinical strains tested being the highest activity against $S$. aureus with an MIC of $12.5 \mu \mathrm{M}$ (Figure 
3.13). However, unlike NSC402959, its structural analogues were toxic to HL-60 cell line compared to controls (section 3.3.1.3.7).

\subsection{Activity of the NSC402959 Analogue NSC15381 against Clinical Strains}

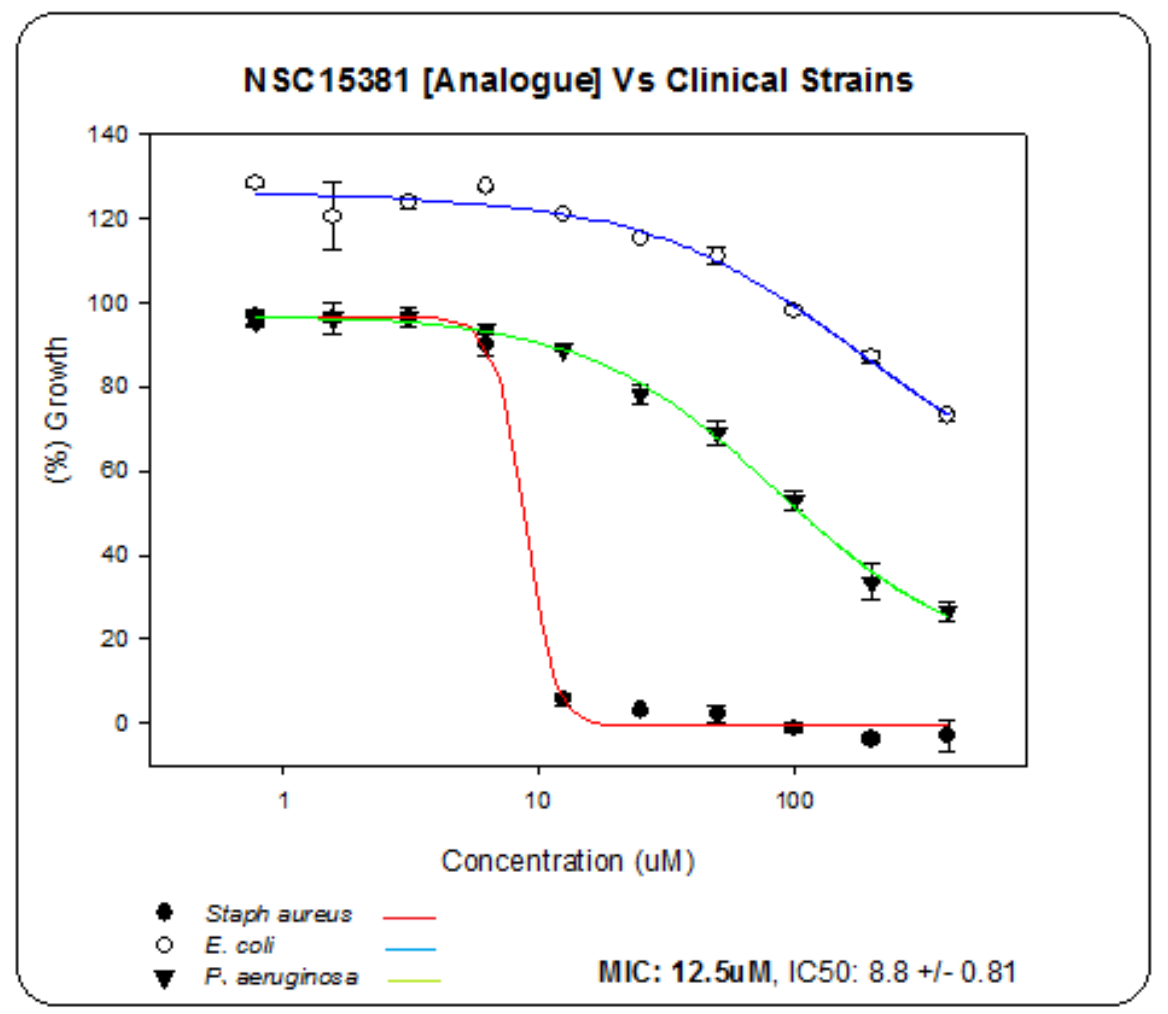

Figure 3.13: Activity of NSC15381 against clinical strains. Unlike NSC402959, its structural analogue NSC15381 showed a better inhibitory activity against $S$. aureus ATCC $25923\left(\mathrm{IC}_{50} 8.8 \mu \mathrm{M} \pm 0.81\right)$ and mild inhibitory activity against P. aeruginosa ATCC 25668 and E. coli ATCC 25922. ( \pm ) sign indicates the standard error between triplicate readings.

\subsection{Cytotoxic Effect of NSC402959 Structural Analogues}

While NSC402959 was not itself cytotoxic to HL-60 cell line, both of its analogues (NSC15381 and NSC603988) showed toxicity against human cells (Figure 3.14). 


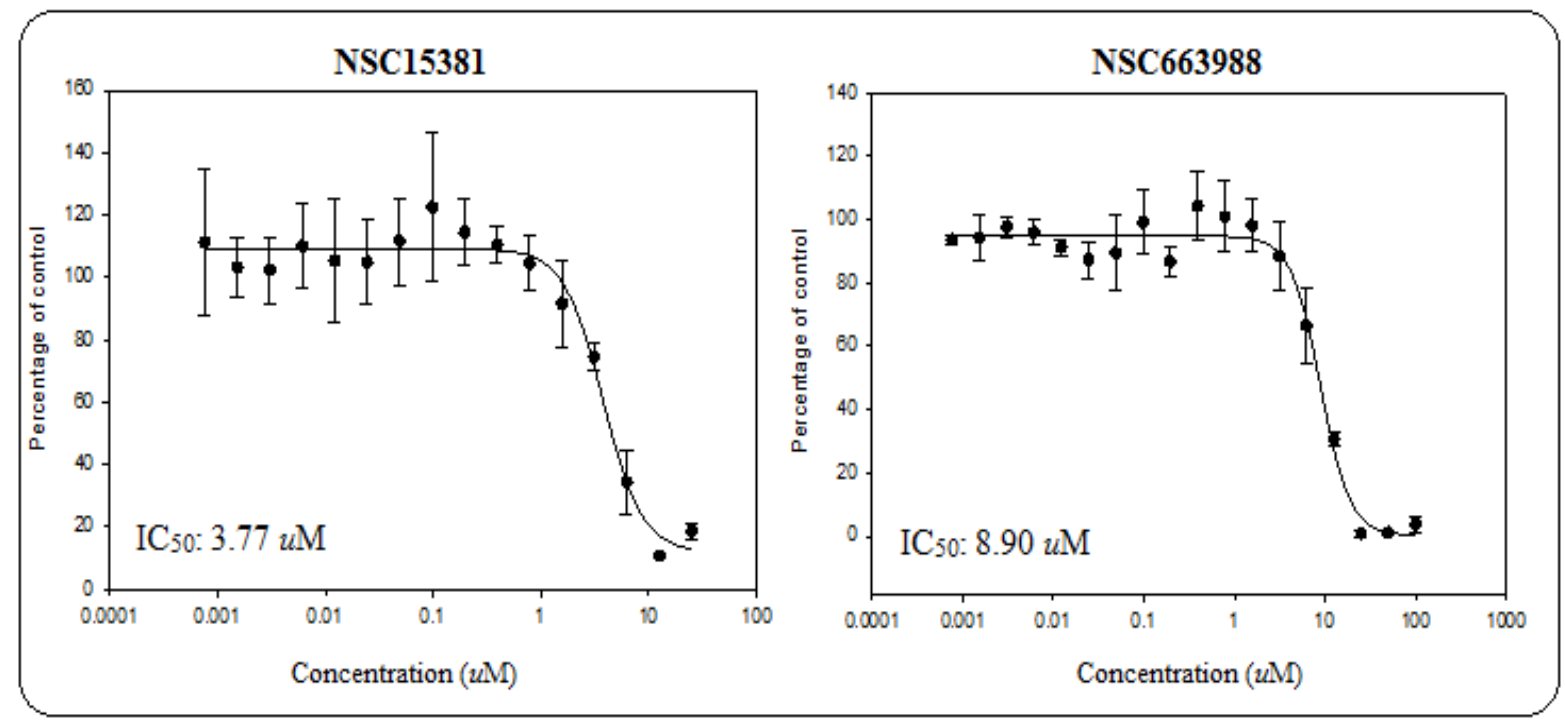

Figure 3.14: Cytotoxicity of NIH compounds against HL-60 cell line. Despite good anti-mycobacterial activity, both compounds NSC15381 and NSC663988 exhibited toxicity towards HL-60 with 50\% inhibitory concentrations of $3.77 \mu \mathrm{M}$ and $8.90 \mu \mathrm{M}$, respectively.

\subsubsection{Library of New Zealand Native Plant Extracts}

Natural products from plants have long been recognised as potential therapeutics and used for various medicinal purposes in history [319-324]. Several plant extracts are known to have a wide range of antimicrobial activity. To test NZ native plants for their anti-mycobacterial activity, a mini library containing more than 50 plant extracts was prepared as described (section 2.6.1, E). Growth inhibition assays for M. bovis BCG were performed as described (section 2.7.2.1). Six plant extracts were found to be active against mycobacteria (Table 3.7). 
Table 3.7: Anti-Tb activity of native New Zealand plant extracts

\begin{tabular}{|c|c|c|c|c|c|c|}
\hline \multirow[t]{2}{*}{ Plant } & \multirow[t]{2}{*}{ Part } & \multirow[t]{2}{*}{ Extract } & \multicolumn{2}{|c|}{$\mathrm{BCG}$} & \multicolumn{2}{|c|}{$M t b$} \\
\hline & & & MIC & $\mathrm{IC}_{50}$ & $\mathrm{MIC}$ & $\mathrm{IC}_{50}$ \\
\hline Lnovae-zealandiae* & Bark & Aqueous & 1.5 & $0.54 \pm 0.03$ & 4.16 & $2.39 \pm 0.33$ \\
\hline L. bullata & Leaf & Methanol & 3.12 & $2.59 \pm 0.43$ & 6.51 & $4.59 \pm 0.32$ \\
\hline M. exelsa & Flower & Methanol & 3.13 & $1.12 \pm 0.12$ & 4.18 & $2.19 \pm 0.76$ \\
\hline P. tenuifolium & Leaf & Ethanol & $>10$ & $5.37 \pm 1.35$ & 1.25 & $0.51 \pm 0.11$ \\
\hline P. crassifolius & Leaf & Methanol & 6.25 & $4.08 \pm 1.76$ & 3.58 & $1.74 \pm 0.18$ \\
\hline P. colorata & Leaf & Methanol & 7.15 & $5.95 \pm 1.28$ & 7.15 & $3.66 \pm 0.39$ \\
\hline \multirow[t]{2}{*}{ Control (rifampicin) } & - & - & 0.12 & $4.94 \times 10^{-5}$ & 2.06 & $4.84 \times 10^{-5} \pm$ \\
\hline & & & x $10^{-3}$ & $\pm 5.02 \times 10^{-6}$ & X $10^{-4}$ & $4.03 \times 10^{-5}$ \\
\hline
\end{tabular}

*Laurelia novae-zealandiae was the only plant species in NZ biota that was previously used for traditional treatment of Tb lesions in Mâori populations [325]. MIC and $\mathrm{IC}_{50}$ values are given in $\mathrm{mg} / \mathrm{mL}$ along with means of standard error $( \pm)$. Only extracts with activity against slow-growing mycobacteria are listed.

New Zealand native plant species have been used previously for a range of medicinal remedies [326]. New Zealand plants extracts were found to be active against mycobacterial strains in this study and their validated anti-tubercular activity against $M$. tuberculosis H37Ra indicated the medicinal potential as well as the diverse flora of this country [241]. Future research will determine their therapeutic efficacy to confirm whether these plant species can be developed as a remedy to treat human infections including $\mathrm{Tb}$.

\subsubsection{96-well Plate Template Used for HTS}

Figure 3.15 shows an HTS template along with a positive hit (at position, 5B) and the arrangement of controls for high throughput screening performed in this study. 


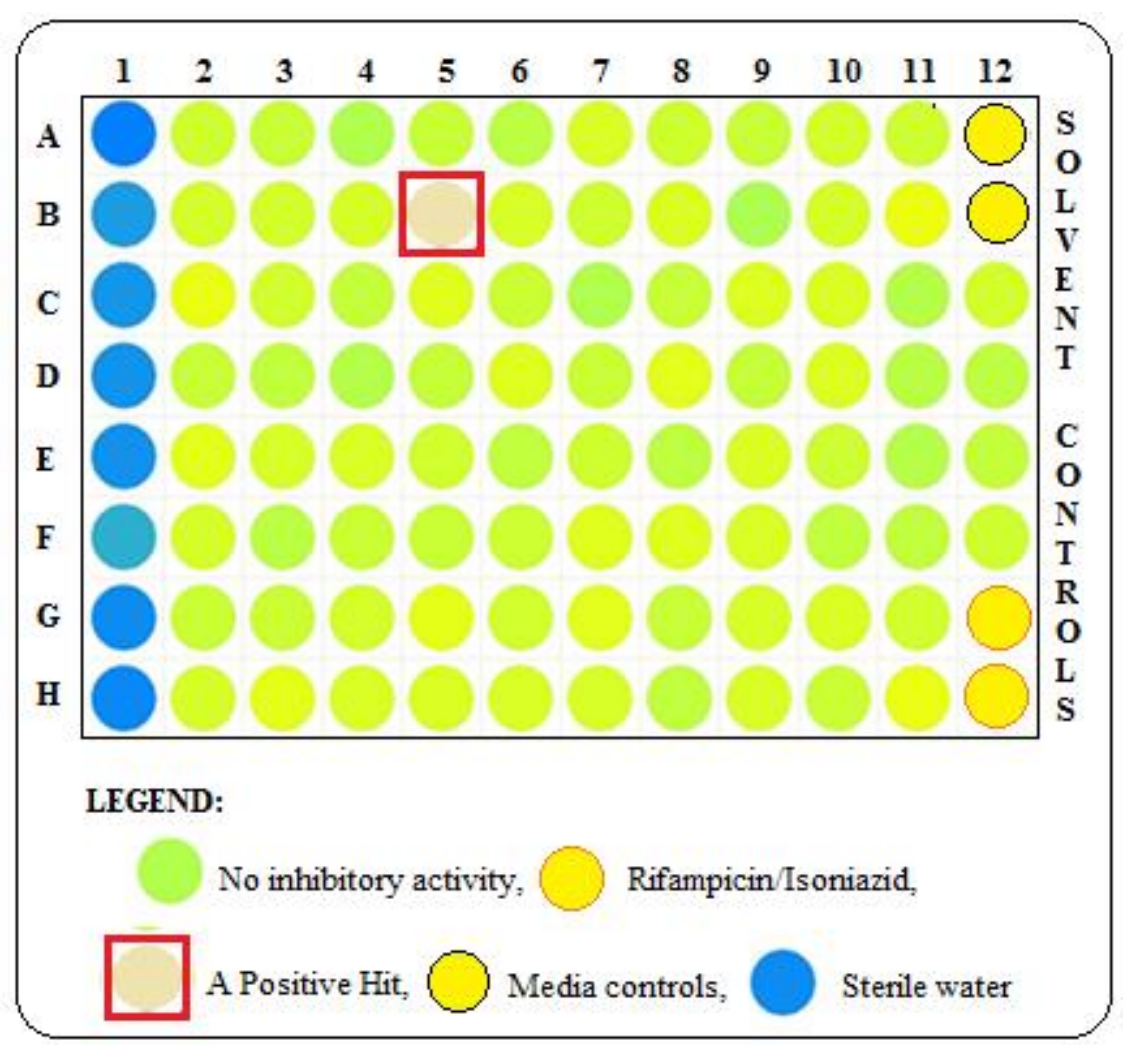

Figure 3.15: A diagram of an HTS 96-well plate. Diagram shows a plate map for controls and a possible hit at plate position 5-B (red square). This compound caused complete growth inhibition of M. bovis BCG (5-B) compared to $100 \%$ growth controls (at positions $12 \mathrm{C}-\mathrm{F}$ ). Cells viability was determined with GFP fluorescence (as shown in green).

\subsubsection{Data Analysis from HTS}

\subsubsection{High-throughput Screening Data Normalization}

Data recovered from HTS were initially processed in Excel spread sheets. All $\mathrm{OD}_{600}$ and GFP fluorescence values were normalized against blanks (without cells), 100\% growth controls and no growth controls in order to get percentage values for each well. 


\subsubsection{Statistical Analysis}

One of the problems with analysing high throughput data is the large amount of data output. Statistical significance was taken into account when analysing the data generated to ensure reliable results as there are often false positive hits in the screens. The signal-to-noise ratio $(\mathrm{S} / \mathrm{N})$ was calculated to remove any outliers that could affect screening results.

\subsection{Z-factor Calculations}

The solvent and 100\% growth controls represented maximum signals while the positive controls (known anti-Tb drugs, rifampicin or isoniazid) yielded minimum-to-no signal. The z-factors for all screens were calculated using the formula below as previously described by Zhang et al. [327].

$$
\mathrm{Z} \text {-factor }=\frac{1-(\text { SD of sample }+ \text { SD of control })}{\text { Mean of sample }- \text { mean of control }}
$$

\subsubsection{Duplicate Screen Correlation}

A duplicate screen was performed against each mycobacterial species, and screening results were directly compared for the total number and types of hits that appeared in both screens. The two data sets were statistically correlated. The $\mathrm{R}^{2}$ value represents a linear correlation between two screens and indicates consistency and reliability of the results from screens performed under identical conditions (Figure 3.16). 


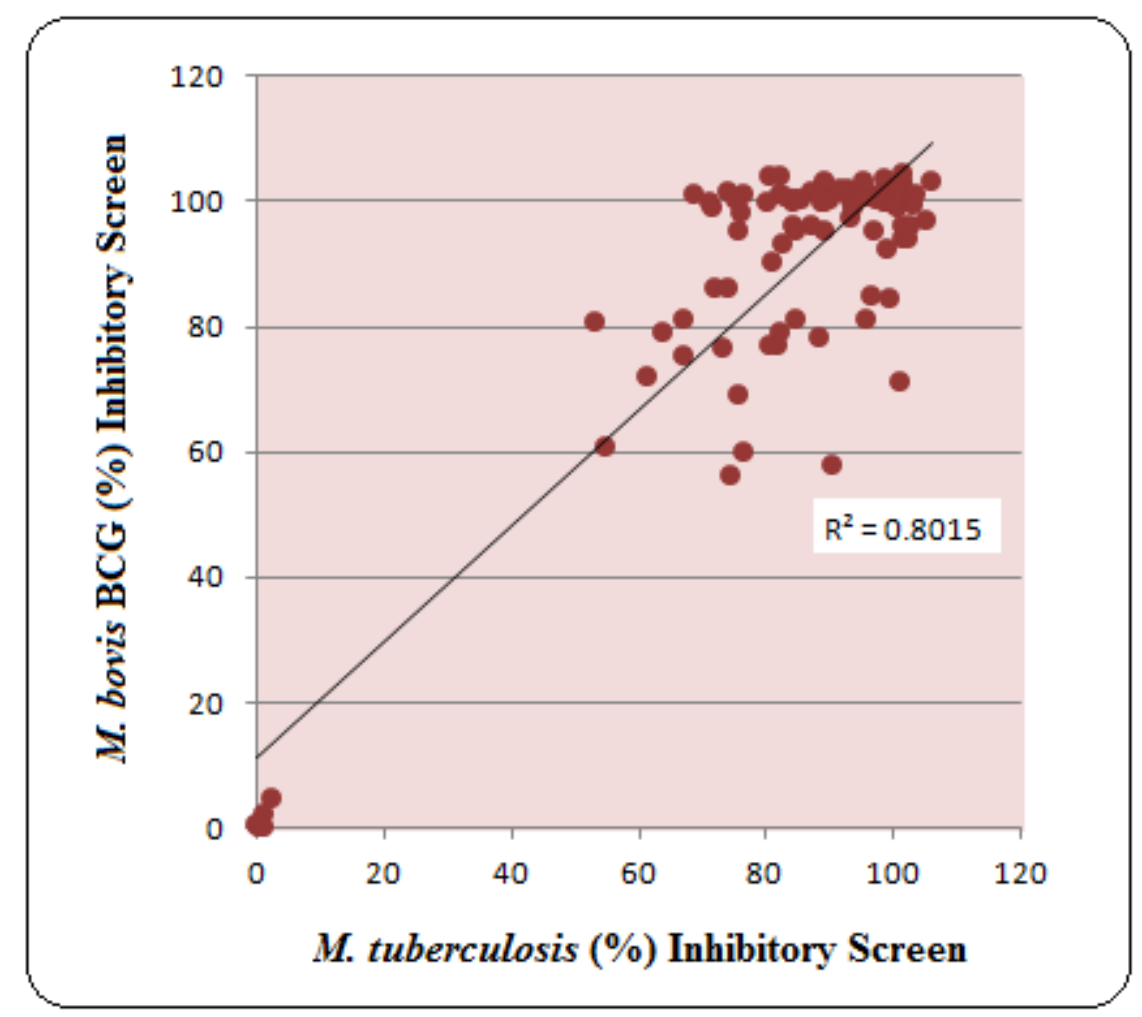

Figure 3.16: Correlation of LOPAC screens performed against M. bovis BCG and M. tuberculosis H37Ra. After taking average $\mathrm{OD}_{600}$ values across rows for each library plate, a significant linear correlation $\left(\mathrm{R}^{2}=\right.$ 0.8015) was observed between the two (data sets) screens performed against both species. Hits that appeared against initial $M$. bovis BCG screens were expected to show similar inhibitory activity when screened against $M$. tuberculosis H37Ra. 
Table 3.8: Comparison of library hits against $M$. bovis BCG and M. tuberculosis

\begin{tabular}{lccc}
\hline Chemical Library & Compounds & \multicolumn{2}{c}{ Total No of hits in HTS $^{1}$} \\
\cline { 3 - 4 } & & M. bovis BCG & M. tuberculosis \\
\hline LOPAC & 1280 & 38 & 37 \\
Spectrum & 1990 & 67 & 62 \\
NIH-DS & 2000 & 76 & 51 \\
NIH-NPS & 240 & 37 & 44 \\
\hline Total Compounds & 5510 & 218 & 194 \\
\hline
\end{tabular}

${ }^{1}$ Comparison of total number of hits that were identified against both species in HTS (an average of triplicate screens). ${ }^{2}$ Diversity Set from National Institutes of Health. ${ }^{3}$ Natural Product Set. (M. bovis BCG hits $3.95 \%, M$. tuberculosis $3.5 \%)$.

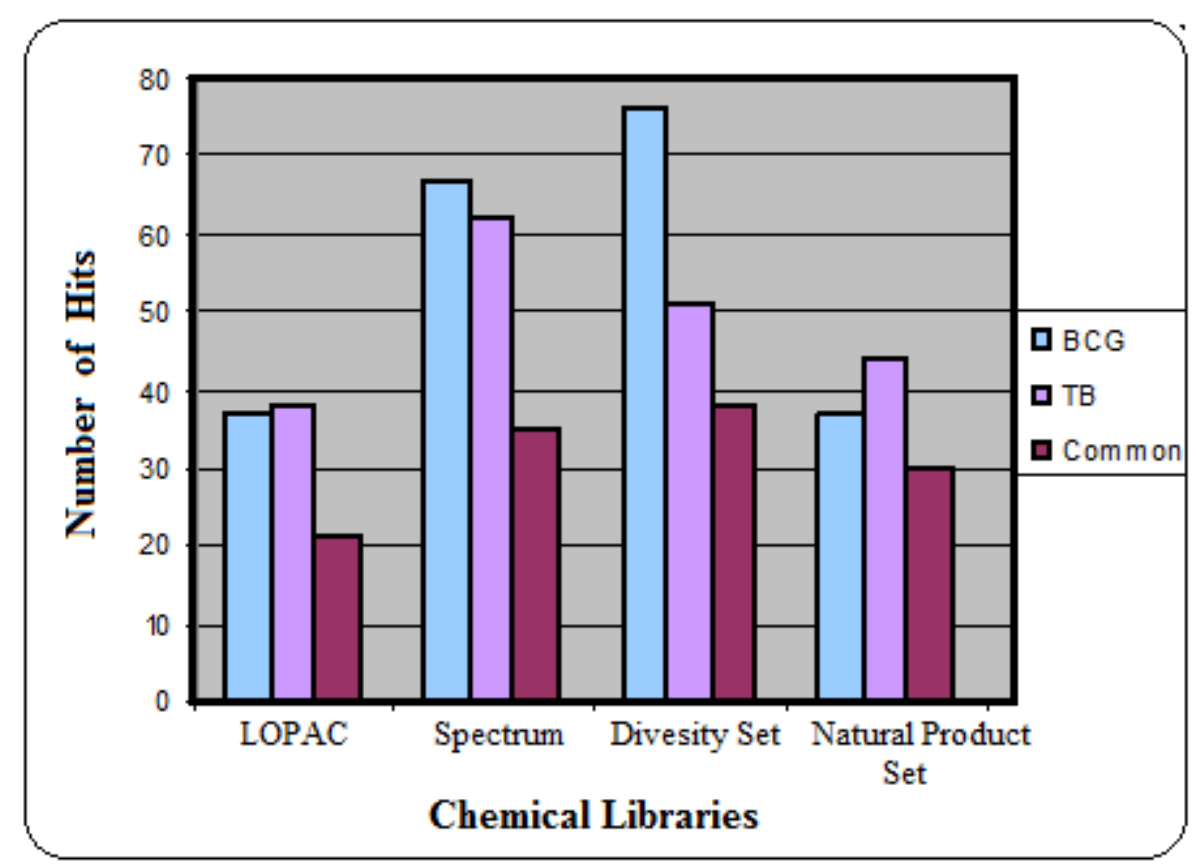

Figure 3.17: Number of common hits between M. bovis BCG and M. tuberculosis H37Ra. More than 60\% of the hits were common between both mycobacterial species. 


\subsubsection{Criteria to Remove Promiscuous Compounds Identified by HTS}

Use of a GFP reporter system and comparing results with $\mathrm{OD}_{600}$ measurements proved useful for establishing a reliable data analysis and correlation, as well as for determining falsepositive hits. If a compound had been identified as a positive hit in previous assays reported in the literature and appeared to have multiple targets or biological activities in humans, such compounds despite their novel anti-tubercular activity, were deemed as interference compounds and were filtered out of our active compound database, as described previously by Jonathan et al. [328].

\subsubsection{Embedded HTS Controls and Tb Screening Models}

Apart from controls and reference drugs used in this study, some previously-reported mycobacterial inhibitors from our lab were used as additional controls for HTS. These include idarubicin, resveratrol, methoctramine, 1-10 phenanthroline and pentamidine [252]. The selection of hits was variable between each Tb models used in HTS (Chapter 4).

\subsubsection{Selection of Leads}

There were many novel compounds identified as potentially active at the completion of HTS listed tables 3.4-3.6. Most hits had a range of biological targets (PubChem database); however, only a few of them were amenable in this research format due to the selection criteria as described (section 3.3.1.1.4) therefore, only a few compounds have been considered appropriate for further characterization. 


\subsection{Discussion}

New anti-Tb compounds that are structurally different from existing drugs and have a novel mechanism of action are needed. Novel drugs would help reducing the lengthy $\mathrm{Tb}$ treatment time [329]. The HTS part of this research has identified several mycobacterial inhibitors that should be considered for further development as novel anti-Tb drugs. Among active anti-M. tuberculosis compounds identified in this study, some inhibitors exhibited significant cytotoxicity towards mammalian cells lines HL-60 and J774 therefore, such inhibitors were excluded from further characterization (section 3.3.1.1.4). However, a few compounds with unique chemical structures and presumably with unique mechanisms of action were identified (e.g. NSC402959, Table 3.6) that were not toxic to human cells compared to positive controls and such compounds were considered to be suitable lead compounds. While identification of inhibitors is the initial step in anti-Tb drug discovery, there are a number of experimental phases involved that enable prioritizing of selected compounds before these can be entered into the development phase [265]. Therefore these hits were further characterized in the present study as potential leads for the development of anti-mycobacterial drugs.

\section{Comparative Activity of NIH Hits with LOPAC and Spectrum Compounds}

NSC402959 was one of interesting compounds detected from the NIH Diversity set in the present study. It showed consistent activity against $M$. tuberculosis H37Ra and displayed low cytotoxicity against human cells $\left(\mathrm{IC}_{50}>150 \mu \mathrm{M}\right)$. NSC402959 was also validated against virulent $M$. tuberculosis strain $(\mathrm{H} 37 \mathrm{Rv})$ and was found to have a similar activity against both the virulent and the avirulent strains (MIC $12.5 \mu \mathrm{M}$ ). Information on NSC402959 from the PubChem database revealed that it inhibited RNase HI from E. coli previously. BLAST analysis of E. coli RNase HI showed approximately $31 \%$ similarity with $M$. tuberculosis RNase HI. After the confirmation of NSC402959's inhibitory activity across a range of 
mycobacterial strains in vitro, it was hypothesized that it may be targeting the same enzyme in M. tuberculosis. This hypothesis was to be tested for further development of a novel class of M. tuberculosis inhibitors (Chapter 5).

\section{Sappanone A Methyl Ether}

Sappanone A dimethyl ether was discovered as a novel anti-mycobacterial compound from the Spectrum library (Table 3.5). It is derived from Caesalpinia sappan L, a flowering tree which is common in South East Asia and belongs to the family Caesalpiniaceae [330]. Compounds extracted from Caesalpinia sappan are Sappanone A/B and Brazilin. Some other compounds have also been isolated from this species that include flavonoids and phenolics, which are responsible for a range of biological activities. Apart from the commercial use of sappanwood, (e.g. paint/ink production from wood extracts) this plant species is known to have anti-bacterial and anti-oxidative activity. However, to our knowledge no previous reports have been made with respect to its specific anti-M. tuberculosis activity.

Biological and therapeutic activities of Caesalpinia sappan L (Brazilin, Sappanone A and B) have been well documented [331]. For example, its traditional medicinal use as an anticonvulsant [332], anti-complementary agent [333], its possible hepatoprotective effect [334] and anti-viral activity [335] have been reported previously. It has also been traditionally used for skin diseases, as an anti-acne agent, and an agent for healing of wounds and skin burns as well as for remedial use for leprosy infections [336-337].

A structural analogue of sappanone A [338], and its derivative 3-deoxysappanone B have also been reported as useful for medicinal purposes in the Encyclopaedia of Traditional Chinese Medicines [339]. Furthermore, Vidavalur et al. have recently evaluated the anti-oxidative potential of sappanone A and have shown that it was better than other commercially-available dietary anti-oxidants such as vitamin $\mathrm{C}$ and vitamin $\mathrm{E}$ and could be used for patients suffering 
from asthma [340]. Other research efforts are underway to further evaluate the medicinal potential of Caesalpinia sappan [341]. Based on its inhibitory effect towards $M$. tuberculosis in the present study (99\% growth inhibition in vitro, Table 3.5), further research is warranted to investigate its specific therapeutic potential for $\mathrm{Tb}$.

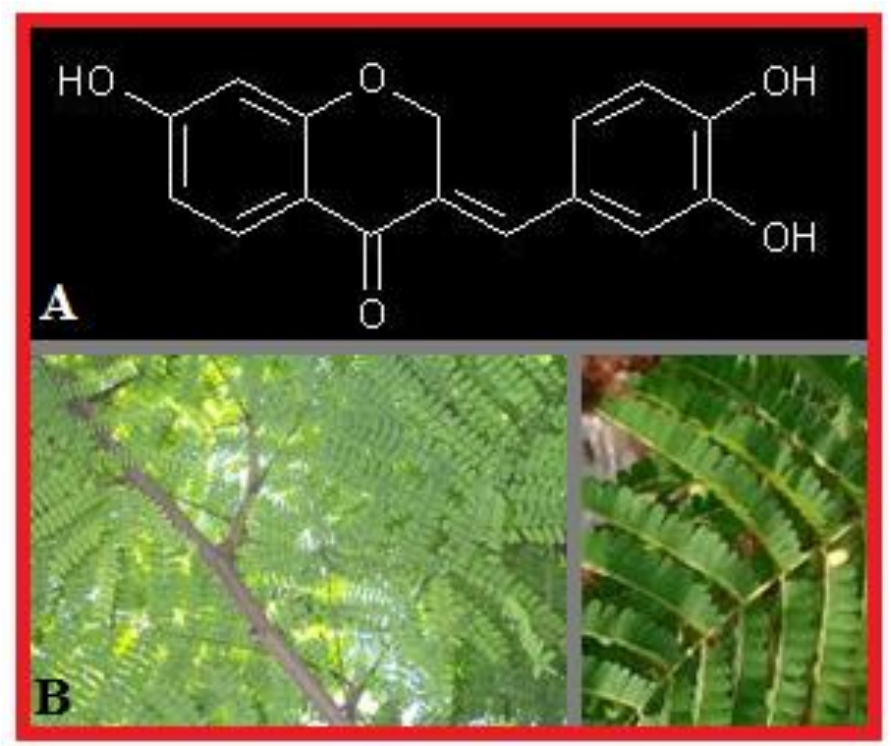

Figure 3.18: Chemical structure of sappanone A, a novel anti-Tb compound identified from the Spectrum library (A) Chemical structure of sappanone A, an active component of Caesalpinia sappan. MW 312.33 g/mol, $\mathrm{MF} \mathrm{C}_{18} \mathrm{H}_{16} \mathrm{O}_{5}$ and (B) Pictures of C. sappan [342].

\section{Key Biological Activity and Importance of Potential Hits}

Several mycobacterial inhibitors identified from the NIH library appeared to be specific in the inhibition of $M$. tuberculosis H37Ra upon validation. For example compounds NSC64111, NSC145611, NSC168466, NSC168467, NSC168468, NSC333003 and NSC402959 exhibited specific anti-M. tuberculosis activity (Table 3.6). None of these compounds were active against M. smegmatis in HTS nor did they show anti-M. bovis BCG activity in the dose response assays. The anti-Tb activity of compounds NSC168466, NSC168467 and NSC168468 may be due to their similar chemical structures (Figure 4.9). However, 
NSC333003 is structurally different from these compounds (PubChem database) yet it exhibited similar level of inhibitory activity against $M$. tuberculosis and there have been no previous anti-Tb data available for NSC333003. These inhibitors were identified as hits against $M$. bovis BCG in HTS, but upon validation experiments they did not exhibit inhibitory activity at concentrations up to $100 \mu \mathrm{M}$ (Table 4.2). Dose-response assays provide a more reliable measure for compound's activity and these $M$. tuberculosis-specific inhibitors were mostly inactive against $M$. bovis BCG upon validation. These compounds were therefore considered as false positive hits in the library screens. However, specific activity against $M$. tuberculosis but not $M$. bovis BCG suggests that there may be unique targets for such inhibitors in M. tuberculosis, given the small genetic difference between $M$. bovis BCG and M. tuberculosis.

Some compounds that are highly active against M. tuberculosis in vitro may not show inhibitory activity against an established infection in vivo. Pethe et al. have presented examples from their study, in which they found $M$. tuberculosis inhibitors in vitro that possessed little or no activity in vivo [343]. While some compounds detected from the LOPAC, Spectrum and NIH libraries in the present study had no previous data for showing activity against $\mathrm{Tb}$, many of them had been previously reported as anti-bacterial. A few inhibitors had recently been reported as anti-tubercular compounds e.g. cloxyquin [311]. However, anti-mycobacterial compounds from NIH library such as NSC333003, which showed marked inhibitory activity against M. tuberculosis, consistently, in at least three independent experiments, had also been reported to be active in a number of other screens including human proteins (PubChem database), suggesting that such inhibitors may not be suitable to be developed into drugs. A group of inhibitors were found to be active against $M$. bovis BCG but not against $M$. tuberculosis, and similarly, some compounds were active 
against $M$. smegmatis but had no activity upon later validation in the $M$. tuberculosis model used in this study (section 4.4.6.2).

\subsection{Conclusion}

In conclusion, after successfully developing a growth inhibition assay for the slow-growing mycobacterial strains (M. bovis BCG and M. tuberculosis H37Ra), a number of $M$. bovis BCG inhibitors were identified that were validated in M. tuberculosis H37Ra. The most promising of these anti-Tb compounds was NSC402959 with no previously reported antimycobacterial activity. Therefore, such compounds were considered to be novel growth inhibitors of $M$. tuberculosis in vitro. Further experimentation on these inhibitors for their anti-M. tuberculosis activity in vivo would help establish whether these inhibitors should be further developed as novel anti-Tb drugs. 


\section{Chapter 4}

Comparison of in vitro Models Used for Anti-Tb Drug Discovery 


\subsection{Introduction}

Due to a leading cause of death and increase in trend of drug-resistant strains [344], Tb drug discovery is one of the greatest challenges today [259, 345-346]. This has led to the search for new and more effective compounds against $\mathrm{Tb}$ and other human infections [347-348]. However, over the last three decades, less than $1 \%$ of 1556 new chemical entities introduced to global market for disease control were dedicated for the treatment of $\mathrm{Tb}$ [349]. An acceleration in the process of anti-Tb drug discovery is clearly needed to strengthen more than 40 years old $\mathrm{Tb}$ treatment regimen with more effective drugs that are also active against persistent $M$. tuberculosis [122]. One of the key aspects towards success of finding a new disease treatment is to have new compounds with novel mechanisms of action [350]. A new mechanism of action means drugs may retain their activity against some of the more resistant Tb strains. To screen for such drugs, this study first determined the applicability of various available in vitro screening models for anti-Tb drug discovery.

\subsubsection{Models for Tb Drug Discovery}

Various experimental models have been used for anti- $\mathrm{Tb}$ drug discovery and related $\mathrm{Tb}$ research to date $[239,351]$. However, each model differs with respect to its sensitivity and reliability for specific mycobacterial inhibitors. While M. tuberculosis H37Ra was used to validate anti-BCG compounds identified (due to lack of a PC3 facility at Victoria University), subsequent validations studies for selective novel inhibitors were performed against $M$. tuberculosis H37Rv at Johns Hopkins Centre for Tuberculosis Research (Figure 4.1). 


\subsubsection{Comparison of H37Ra and H37Rv Strains}

While $M$. tuberculosis H37Ra has been considered a suitable model organism in primary screening to identify compounds that are likely to show anti-Tb activity [352], a close correlation in drug susceptibility data was observed in this study between $M$. tuberculosis H37Ra and M. tuberculosis H37Rv (Figure 3.12 and Figure 4.12). Based on the previous drug susceptibility reports [352-353] and a high genome similarity between $M$. tuberculosis H37Ra and M. tuberculosis H37Rv (98.9\% of H37Rv genes have orthologues in H37Ra using a protein similarity cut-off of 50\%), a good correlation between these two strains in library screens was expected (section 4.3). Since it is apparent from the available data that $M$. tuberculosis H37Ra could be a good model for M. tuberculosis H37Rv in drug screening, it was selected as one of the reference strains in this work, in conjunction with the other screening models of a slow growing species, $M$. bovis BCG and a fast growing species, $M$. smegmatis (Table 2.1 and Table 3.2). 


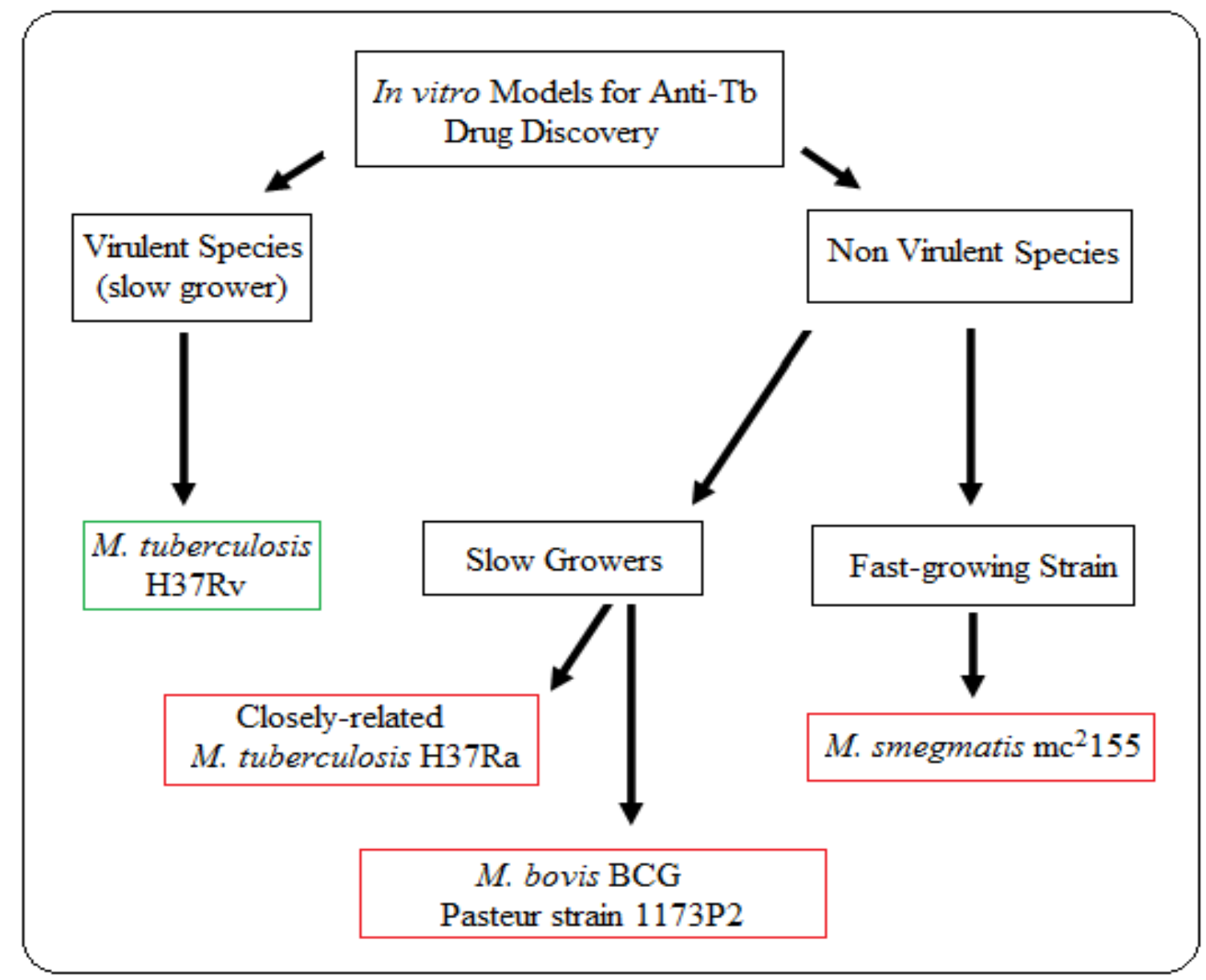

Figure 4.1: In vitro models for anti-Tb drug screening. Three avirulent Tb models $M$. smegmatis mc2155, $M$. bovis BCG Pasteur 1173P2 and M. tuberculosis H37Ra (red) were employed in this study (PC2 lab at VUW) including validation studies by using H37Rv (green) in a PC3 facility at Johns Hopkins Centre for Tuberculosis Research. 


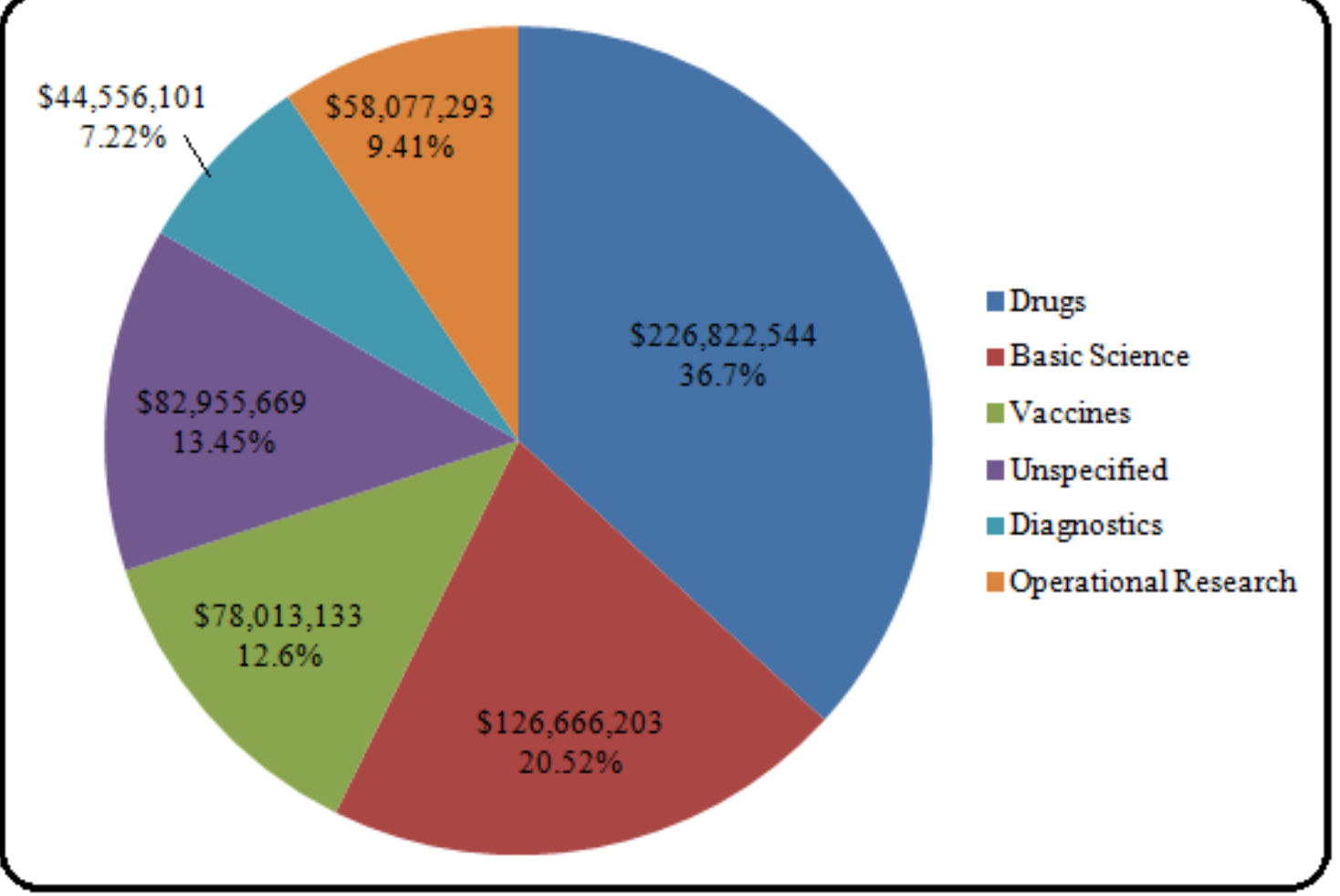

Figure 4.2: Allocated funding for Tb research and development in 2010. Over 226 million US dollars were spent on the search for new drugs $(36.7 \%)$ to cure $\mathrm{Tb}$, which was more than any other Tb-related funding in the year 2010 [354].

The above statistical figure indicates the urgency for the search of new drugs for treating and containing $\mathrm{Tb}$ infections worldwide. To conduct this research, an appropriate in vitro model is required for reliable screening of potential inhibitory compounds.

\subsection{Goals of This Study}

1. To evaluate the efficacy of the M. smegmatis model for the discovery of M. tuberculosis growth inhibitors.

2. To determine the sensitivity of $M$. bovis BCG as a reliable screening model for drug discovery. 


\subsection{Comparative Genomic Analysis}

\subsubsection{M. tuberculosis H37Rv Genome}

M. tuberculosis $\mathrm{H} 37 \mathrm{Rv}$ is the widely used laboratory strain in $\mathrm{Tb}$ research due to its virulence in vivo and drug susceptibility [355]. The complete sequencing of $M$. tuberculosis $\mathrm{H} 37 \mathrm{Rv}$ genome was accomplished in 1998 [17] and has undoubtedly helped researchers to understand $\mathrm{Tb}$ pathology and to develop efficient $\mathrm{Tb}$ diagnostic tools [356]. M. tuberculosis $\mathrm{H} 37 \mathrm{Rv}$ has a GC rich genome (GC and AT contents $65.6 \%$ and $34.4 \%$ respectively) with over 4000 protein-coding genes (Figure 4.3) and is capable of synthesizing essential enzymes, co-factors and amino acids required for its growth under aerobic and anaerobic conditions [17-18]. M. tuberculosis genome sequences and annotations (first performed by Cole et al. [17]), has facilitated the development of new drugs and identification of their respective cellular targets.

\subsubsection{M. bovis BCG Genome Comparison with M. tuberculosis}

$M$. bovis genome was sequenced in 2003 and bears more than $99 \%$ similarity with $M$. tuberculosis at DNA level [56]. M. bovis BCG Pasteur 1173P2 is the Tb vaccine strain developed through repeated in vitro passages of $M$. bovis (section 1.3.2.1). Its complete genome sequencing was published in 2007 (Figure 7.13), which contains 3954 protein-coding genes [357] compared to 4034 protein-coding genes in H37Ra. Since the establishment of $M$. bovis BCG as a vaccine, it has been used for a number of research purposes [358-360] including drug susceptibility testing and random screening for inhibitors [361-363]. M. bovis BCG possesses a higher genome similarity with $M$. tuberculosis species than other screening models used for anti-Tb drug discovery (section 4.4.8). While a high percentage of proteincoding genes are common between $M$. bovis BCG and M. tuberculosis H37Ra, approximately 
$30 \%$ of $M$. tuberculosis genes lack conserved orthologues in M. smegmatis compared to only 3\% in $M$. bovis $\mathrm{BCG}$, making $M$. bovis $\mathrm{BCG}$ the preferred choice for modelling the $\mathrm{Tb}$ bacterium as compared to $M$. smegmatis. Furthermore, due to a closer genetic relationship with M. tuberculosis and non-virulent nature of M. bovis BCG [364-365], it was considered as an apt model system for the screening of $M$. tuberculosis inhibitors. This study was therefore designed to examine if $M$. bovis BCG is more sensitive and relevant than $M$. smegmatis for modelling M. tuberculosis. Growth conditions and culture requirements for these species are described in section 2.2.

M. bovis BCG has three large genomic deletions (regions of difference) referred to RD1, RD2 and RD3 compared to M. tuberculosis H37Rv (section 1.3.2.1). Mahairas et al. have evaluated these genetic differences between strains and have identified the sizes of (RD1RD3) genomic deletions to be $9.5 \mathrm{~kb}, 10.7 \mathrm{~kb}$ and $9.3 \mathrm{~kb}$, respectively [62]. Unlike M. bovis $\mathrm{BCG}$, genomic regions (covering RD1-RD3) are present in $\mathrm{H} 37 \mathrm{Rv}$ genome and are conserved in other virulent mycobacterial species. RD1 encodes for several mycobacterial regulatory factors mainly associated with virulence (secretary antigens) such as ESAT6 and CFP10 (expressed by genes present in RD1), whose restoration in M. bovis BCG results in restoration of a protein profile nearly identical to $M$. bovis and M. tuberculosis H37Rv [62-63, 366]. RD1 sequence can therefore be used as a target to differentiate $M$. bovis BCG from other MTBC species through PCR [367]. These large deletions of genomic regions are associated with mycobacterial survival and growth in vivo [368] and may even provide resistance to some drugs. In vitro evidence suggests that $M$. bovis BCG containing RD2 (M. bovis BCG-Japan, BCG-Russia and BCG Moreau) were resistant to macrolides compared to other macrolide-susceptible BCG strains including BCG-Pasteur, which did not had RD2 [369]. Previous studies do suggest multi-drug efflux activity in M. tuberculosis [201, 358], and more recently, a regulator of mycobacterial efflux pump (Rv3066) has been described by 
Bolla and Do et al. [370]. These pumps are associated with mycobacterial virulence as they play a vital role in conferring drug resistance by up regulating genes that encode for efflux pump and membrane proteins. Mutation in $r p o B$ ( $\beta$-subunit of RNA polymerase) gene leads to rifampicin resistance in $M$. tuberculosis has recently been associated with efflux [371-372], a similar efflux-related activity is responsible for pyrazinamide resistance [373-374]. It is therefore believed that efflux pumps are used to export drugs out of the cell thereby conferring intrinsic resistance to antibiotics [375], e.g. Rv1285c gene (encodes for the tap efflux pump) in M. bovis BCG is responsible for altered drug susceptibility and for inducing phenotypic change that affects cell growth [376]. Inhibitors that are able to block the activity of multi-drug efflux pumps can reverse the situation [358, 377-378]. However, an inhibitor has to evade the bacterial efflux pumps and get through the cell wall barriers to be able to effectively inactivate its cellular target [379], particularly an anti-mycobacterial agent [380]. Since, enhanced expression of genes encoding for multi-drug efflux pumps may extrude a wide range of drugs $[376,381]$, therefore, this should be considered while selecting lead compounds for in vivo studies. 


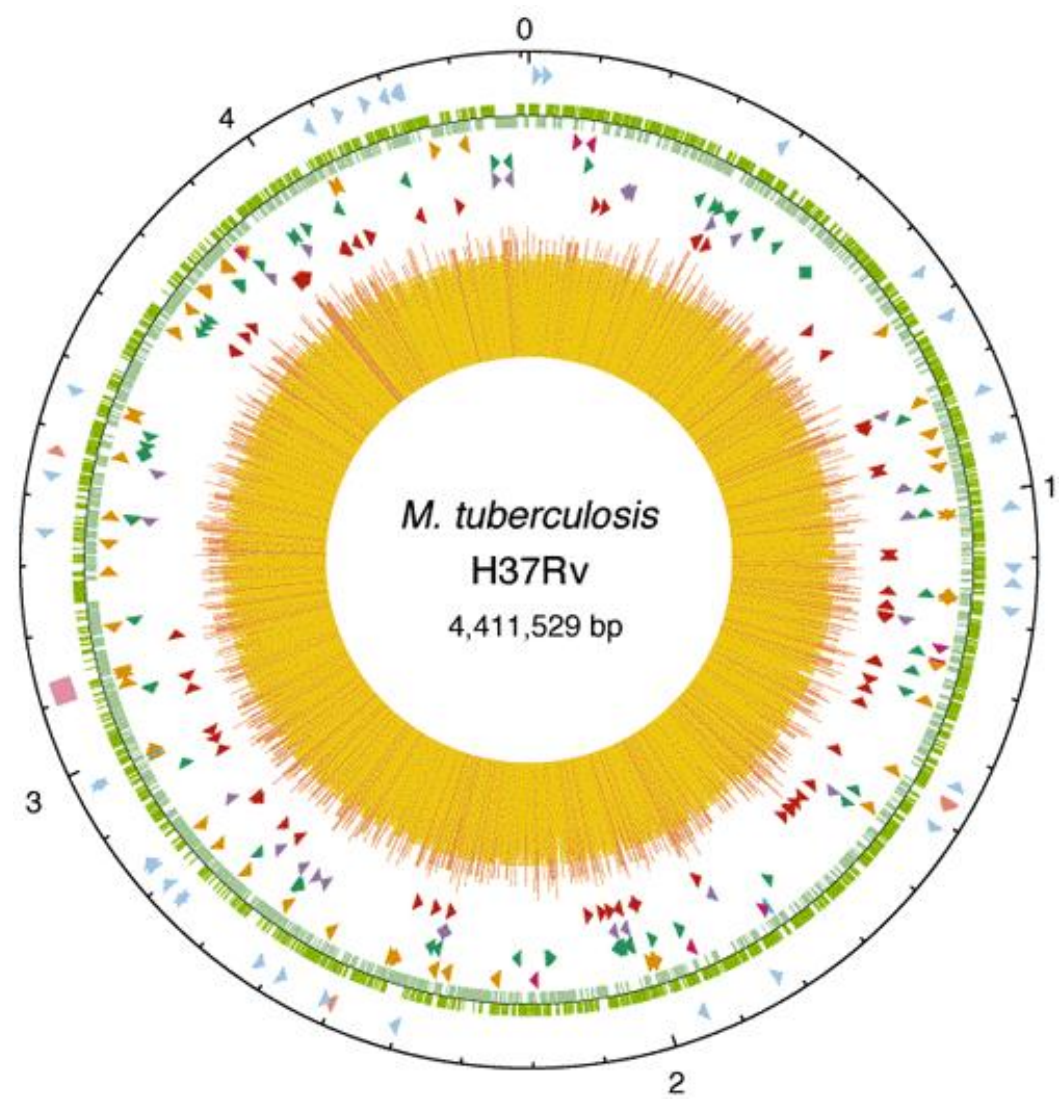

Figure 4.3: The genome of M. tuberculosis H37Rv. Figure reproduced with permission from Cole et al. [17].

\subsubsection{M. smegmatis Controversy in the Tb Field}

M. smegmatis is a fast growing mycobacterial species which replicates every 2-3 hours [382].

It has been used with a number of other species for in vitro modelling in Tb research [239, 383]. M. smegmatis is a saprophytic mycobacterial species [384], and has been widely used in Tb research [385-390]. A letter entitled "Mycobacterium smegmatis: an absurd model for tuberculosis?" [391] provoked a useful discussion with respect to M. smegmatis applicability in studying $\mathrm{Tb}$ [392-393]. Furthermore, lack of $M$. smegmatis ability to survive within macrophages [394-396] and to cause a significant virulence within in vivo models have been shown previously [397], controversy therefore appeared to persist with respect to its ability to mimic the hallmark of Tb. 
Although widely used as a convenient model organism, few if any studies have been conducted to determine the efficacy of $M$. smegmatis in drug screening. In this study, a direct comparison of $M$. smegmatis with another Tb model, M. bovis BCG was performed to detect novel inhibitors of $M$. tuberculosis by screening chemical libraries. Parallel screens were performed against a third $\mathrm{Tb}$ model, M. tuberculosis $\mathrm{H} 37 \mathrm{Ra}$ under identical growth conditions, and the data produced from HTS were analysed to determine similarities and differences between these screening models (section 4.4).

\subsection{Results}

\subsubsection{Results from Library Screens}

Three compounds libraries (section 2.6) were screened to identify inhibitors of mycobacterial growth. The anti-mycobacterial compounds identified with each $\mathrm{Tb}$ models were then compared for similarities.

\subsubsection{Comparison of M. tuberculosis Hits Identified Using Different Tb Models}

The inhibitors detected in HTS of the compound libraries using the three Tb models used in this study (Figure 4.1) were compared. The compounds were grouped on the basis of their inhibitory activity against one or more of the mycobacterial species. Using the selection criteria outlined in section 3.3.1.1.4, a total of 14, 27 and 24 hits were detected against $M$. smegmatis, $M$. bovis BCG and $M$. tuberculosis, respectively using the LOPAC library (Figure 4.4). In the Spectrum Collection 36, 65 and 45 hits were obtained (Figure 4.7) and in the NIH Diversity Set 69, 126 and 91 hits were detected (Figure 4.8). 
After analysing the data from the screens, it was obvious that a majority of antimycobacterial inhibitors identified using the LOPAC library against M. smegmatis $(\mathrm{n}=12$, 85.7\%) were also detected as being active against $M$. tuberculosis. However, a significant number of anti-M. tuberculosis compounds were not detected using $M$. smegmatis in the LOPAC library. At least $50 \%$ of the hits $(\mathrm{n}=12)$ were missed in the screens with $M$. smegmatis as a model organism for the detection of anti-mycobacterial compounds. A different situation existed for M. bovis BCG as only 5 hits (21\%) of the M. tuberculosis hits were not detected with $M$. bovis BCG from the LOPAC library. Furthermore, the percentage of hits that were common between M. bovis BCG and M. tuberculosis H37Ra (33\%) was higher than the number of hits $(4 \%)$ that were common between $M$. tuberculosis and $M$. smegmatis (Figure 4.4). These results confirm M. bovis BCG as a more suitable model of $\mathrm{Tb}$ than M. smegmatis.

To examine if the above findings were unique to LOPAC, the other two sets of chemical libraries, the Spectrum collection and the NIH Diversity Set were also screened against all model organisms. An even higher number of M. tuberculosis H37Ra hits in the Spectrum Collection were absent in M. smegmatis HTS i.e. 55.5\% of the M. tuberculosis H37Ra (n=26) and $48.3 \%(n=44)$, respectively. However, the majority of inhibitors identified in these two compound libraries with $M$. smegmatis were also detected as active against $M$. tuberculosis H37Ra. Thus $50 \%$ of the M. smegmatis hits from the Spectrum collection were also seen with M. tuberculosis $(\mathrm{n}=18)$ and $68 \%$ of the hits from the NIH library were also seen in $M$. tuberculosis $(\mathrm{n}=47)$. Only $13.3 \%$ of $M$. tuberculosis hits $(\mathrm{n}=6)$ from the Spectrum Collection and $6.6 \%(\mathrm{n}=6)$ from the NIH Diversity set were not also detected with $M$. bovis BCG. These data again suggest that the ability of $M$. bovis BCG to identify inhibitors in HTS that are also active against $M$. tuberculosis is better than M. smegmatis (Figures 4.7 and 4.8). 


\subsubsection{Dose-response Assays for the Validation of Mycobacterial Inhibitors}

All dose-response assays for validation of HTS hits were performed as described in section 2.7. Compounds identified from LOPAC and NIH screens were validated for their inhibitory activity against all three model species. Compounds yielding an MIC of $<25 \mu \mathrm{M}$ against $M$. tuberculosis from the LOPAC and NIH Diversity set are listed in tables 4.1 and 4.2, respectively. Individual examples of compounds that were not detected with $M$. smegmatis but were identified with $M$. bovis BCG and inhibitory activity was validated in $M$. tuberculosis are noted in sections 4.4.4 and 4.4.6.

\subsubsection{LOPAC Compounds}

Hits obtained from the screens against the three mycobacterial species were compared and a Venn diagram was drawn to examine the difference between the ability of each species to detect M. tuberculosis inhibitors (Figure 4.4). Mycobacterial inhibitors identified from the LOPAC library that were detected by the slow growers but were not detected with $M$. smegmatis are listed in table 4.1. These inhibitors included ammonium pyrrolidine dithiocarbamate (APDC), 2-chloroadenosine, fusidic acid and tetraethylthiuram disulphide (TETD). APDC was detected only when $M$. bovis BCG was used in the screens and it was also validated against $M$. tuberculosis. MICs for APDC against $M$. bovis BCG and $M$. tuberculosis were $1.56 \mu \mathrm{M}$ and $0.781 \mu \mathrm{M}$ respectively (Figure 4.5). There was no anti- $M$. smegmatis activity of APDC observed at the highest concentration tested (100 $\mu \mathrm{M})$ (Table 4.1). Similarly, TETD was not detected with M. smegmatis but M. bovis BCG, and was validated in M. tuberculosis H37Ra (Figure 4.6).

Niclosamide is a known inhibitor of $M$. tuberculosis, but it was not detected with $M$. smegmatis in this study. However, it did exhibit inhibitory activity against $M$. smegmatis in 
dose-response experiments with an MIC of $25 \mu \mathrm{M}$. It was only identified in the $M$. bovis BCG HTS and it showed good activity against both M. bovis BCG and M. tuberculosis in a validation experiment with an MIC of $6.25 \mu \mathrm{M}$ against both Tb strains (Table 4.1).

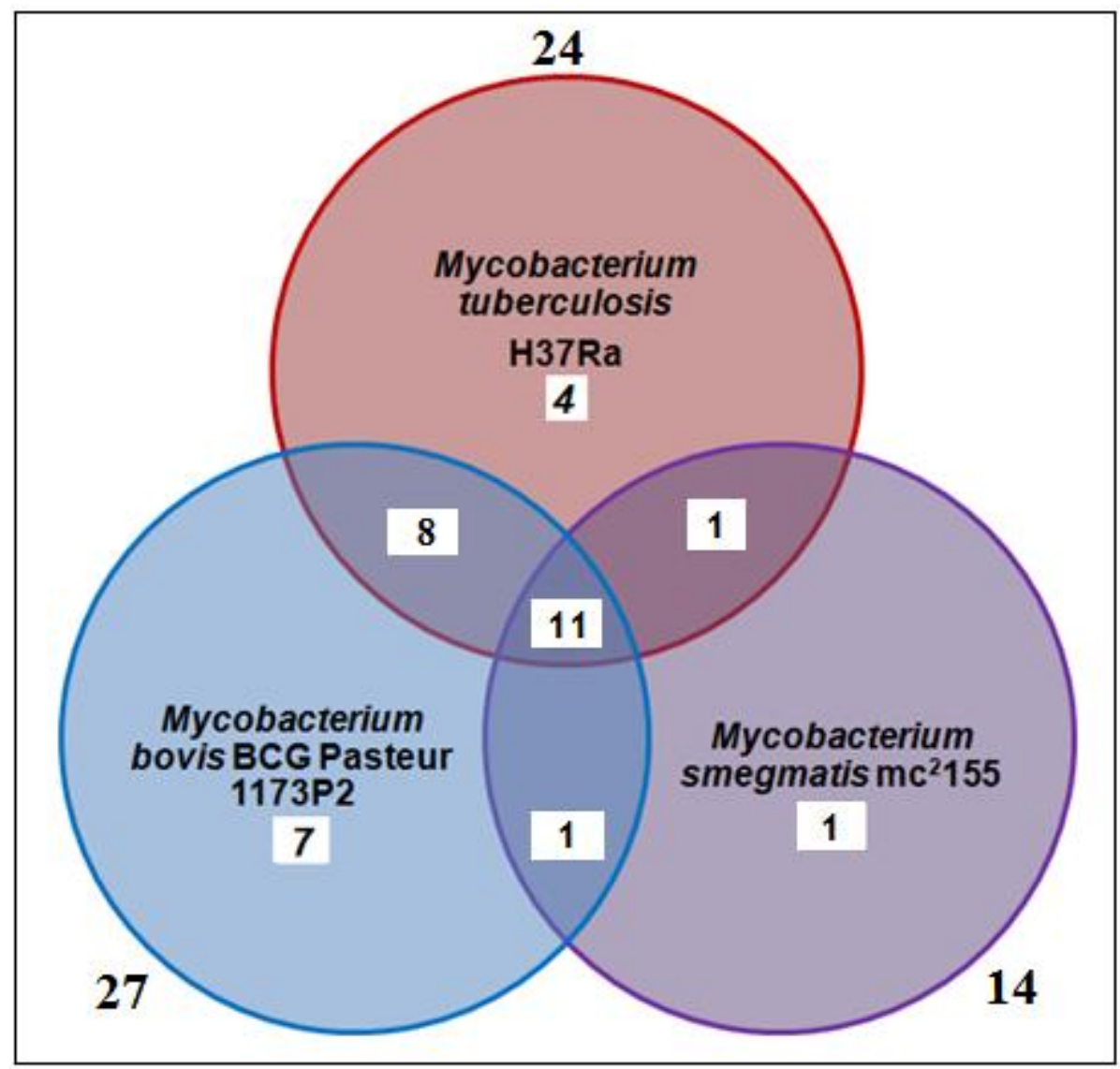

Figure 4.4: Venn diagram for the LOPAC library showing the distribution of mycobacterial compounds detected with M. smegmatis $\mathrm{mc}^{2} 155$, M. bovis BCG Pasteur 1173P2 and M. tuberculosis H37Ra. A total of 14, 27 and 24 hits were detected against M. smegmatis, M. bovis BCG and M. tuberculosis H37Ra, respectively, in the LOPAC library. 
Table 4.1: Validated mycobacterial inhibitors from the LOPAC library

\begin{tabular}{|c|c|c|c|}
\hline \multirow[t]{2}{*}{ LOPAC Compound } & \multicolumn{3}{|c|}{ MIC Values $(\mu \mathrm{M})$} \\
\hline & smegmatis & M. bovis & M. tuberculosis \\
\hline Ammonium pyrrolidine dithiocarbamate & $>100$ & $\underline{1.56}$ & $\underline{0.781}$ \\
\hline 2-Chloroadenosine & $>100$ & $\underline{12.5}$ & $\underline{12.5}$ \\
\hline Demeclocyclin dichloride & 3.125 & 3.125 & 1.56 \\
\hline Dequalinium dichloride & 3.125 & 3.125 & 6.25 \\
\hline Diphenyleneiodonium chloride & 3.125 & 0.195 & 0.39 \\
\hline Ellipticine & 25 & 12.5 & 12.5 \\
\hline Fusidic acid sodium & $>100$ & $\underline{3.125}$ & $\underline{1.56}$ \\
\hline Idarubicin & 3.125 & 6.25 & 6.25 \\
\hline Lomefloxacin hydrochloride & 6.25 & 1.56 & 3.125 \\
\hline Methoctramine hydrochloride & 12.5 & 1.56 & 6.25 \\
\hline Minocycline hydrochloride & 12.5 & 1.56 & 3.125 \\
\hline Niclosamide & 25 & $\underline{6.25}$ & $\underline{6.25}$ \\
\hline Ofloxacin & 3.125 & 1.56 & 1.56 \\
\hline Tetraethylthiuram disulphide & $>100$ & $\underline{3.125}$ & $\underline{6.25}$ \\
\hline WB-64 & 12.5 & 12.5 & 12.5 \\
\hline
\end{tabular}

Underlined MIC values indicate M. tuberculosis inhibitors that were detected with M. bovis BCG during screening but not with M. smegmatis. Compounds were tested up to a maximum concentration of $100 \mu \mathrm{M}$ across all species. Only LOPAC compounds which yielded an MIC of $12.5 \mu \mathrm{M}$ against $M$. tuberculosis upon validation in dose-response assays are listed. 


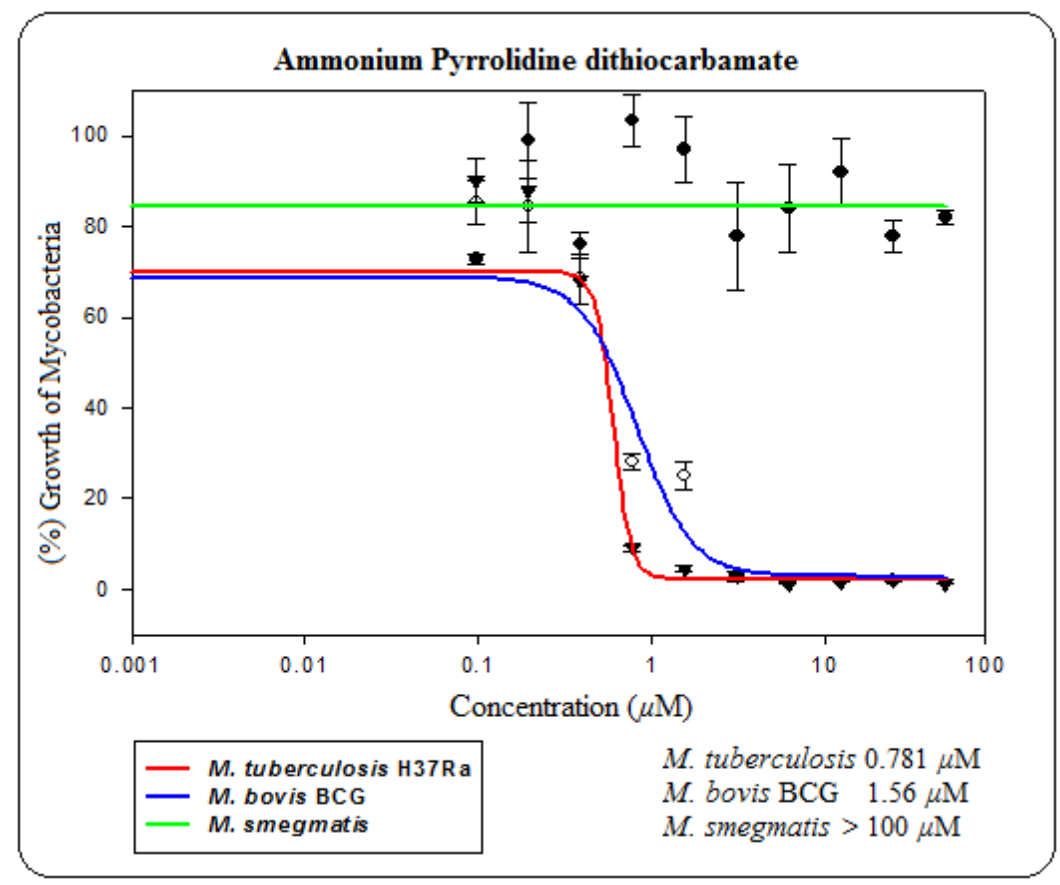

Figure 4.5: Dose-response curve of ammonium pyrrolidine dithiocarbamate against mycobacteria. Three mycobacterial species are M. smegmatis mc2155 (green), M. bovis BCG Pasteur 1173P2 (blue) and M. tuberculosis H37Ra (red).

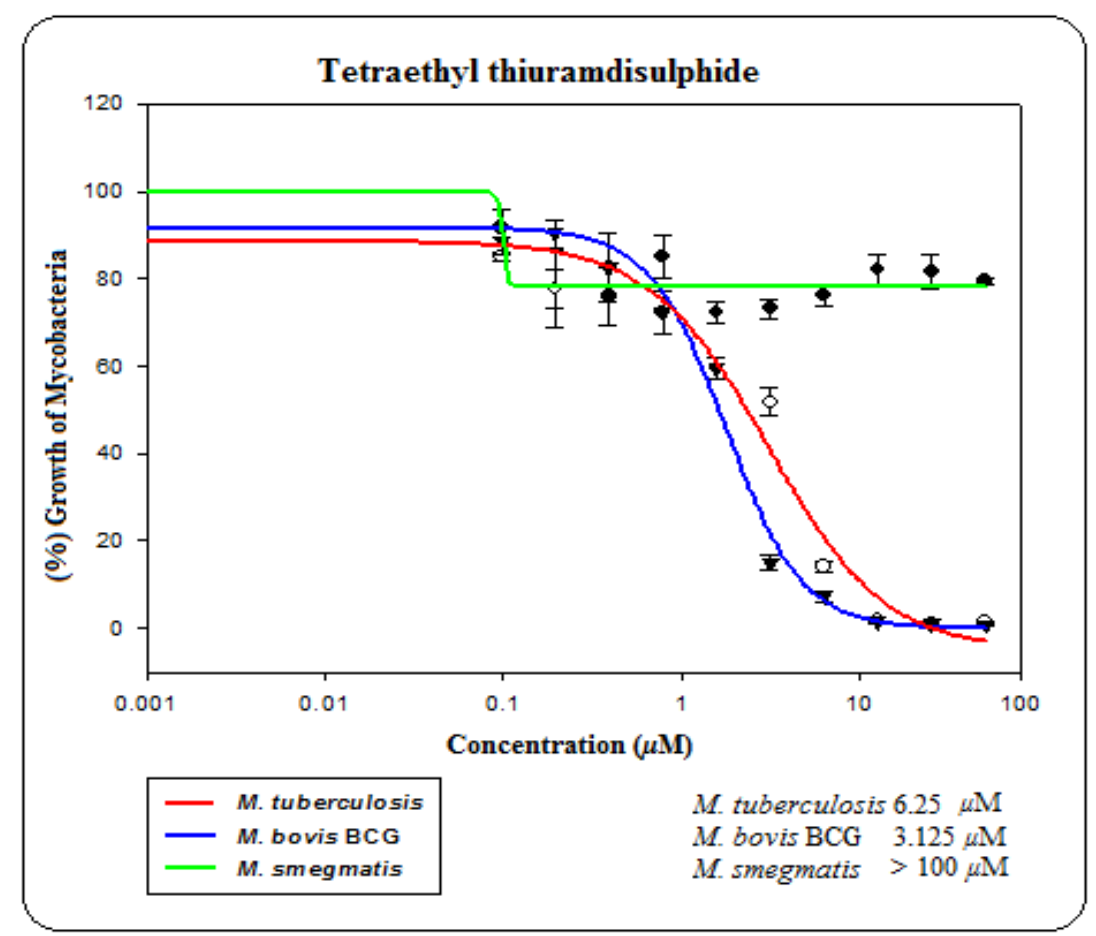

Figure 4.6: Dose-response curve of tetraethyl thiuramdisulphide (TETD) against mycobacteria, $M$. smegmatis mc2155 (green), M. bovis BCG Pasteur 1173P2 (blue) and M. tuberculosis H37Ra (red). There was 
no inhibitory activity of TETD observed against $M$. smegmatis at concentrations up to $100 \mu \mathrm{M}$. TETD was identified in the M. bovis BCG screen (MIC $3.125 \mu \mathrm{M}$ ) but not with M. smegmatis screen (MIC >100 $\mu \mathrm{M}$ ) and was validated against $M$. tuberculosis $\mathrm{H} 37 \mathrm{Ra}(\mathrm{MIC} 6.25 \mu \mathrm{M})$.

\subsubsection{Spectrum Collection}

Sappanone A was identified as a hit during Spectrum screens using $M$. bovis BCG. It was also identified as an active compound in the M. tuberculosis screen but was not detected with M. smegmatis. Sappanone A dimethly ether has no previously reported anti-M. tuberculosis activity and may therefore be a potential novel candidate for further development as an antiTb agent (section 3.3.1.2.4). These differences in the identification of M. tuberculosisspecific inhibitors using different $\mathrm{Tb}$ models demonstrate the importance of selecting an appropriate in vitro $\mathrm{Tb}$ model prior to screen for active inhibitors. 


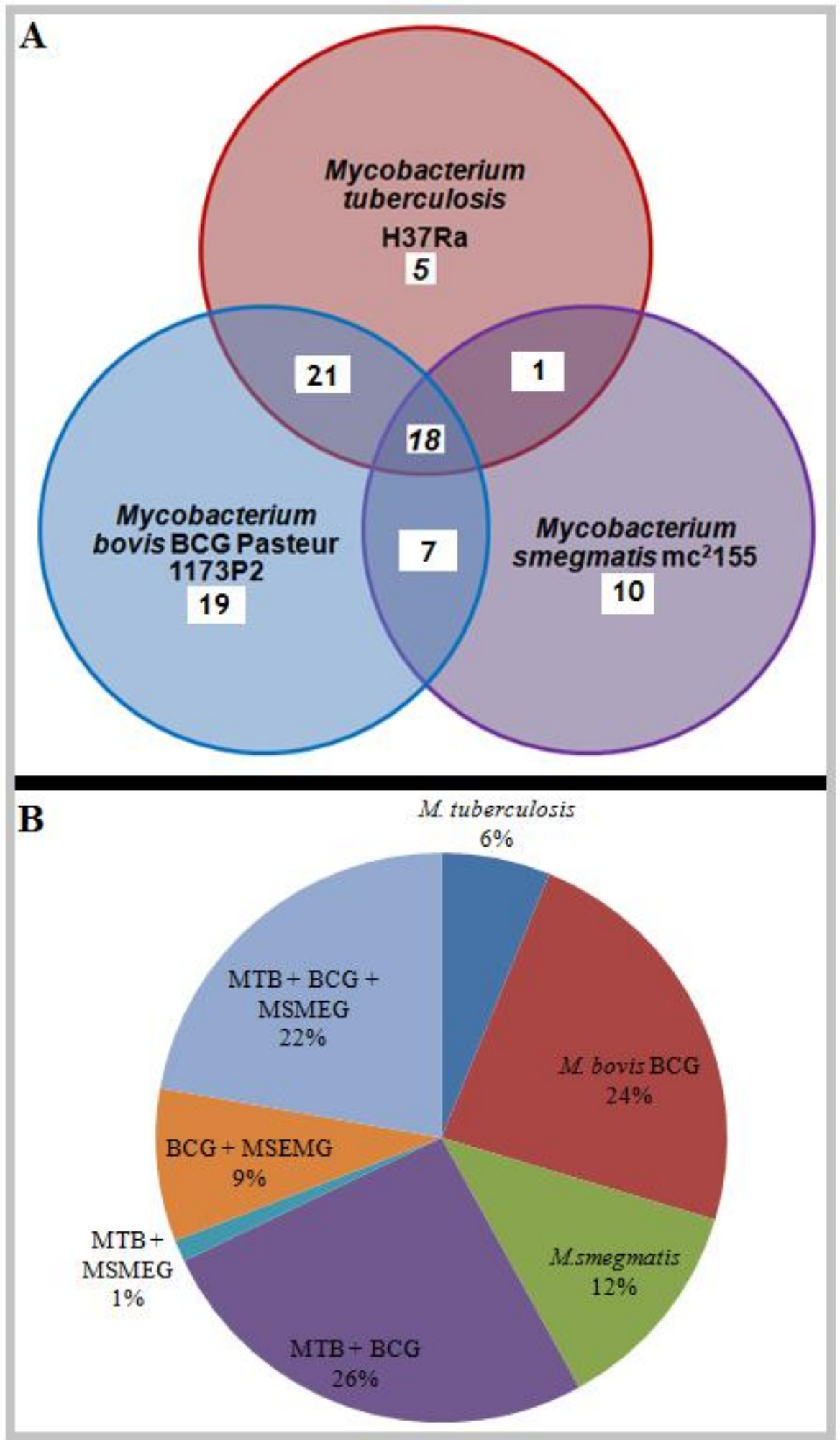

Figure 4.7: Venn diagrams for Spectrum library (A) Number of hits identified across the three mycobacterial species, M. smegmatis mc ${ }^{2} 155$ (36 hits), M. bovis BCG Pasteur 1173P2 (65 hits) and M. tuberculosis H37Ra (45 hits) and (B) Percentage of inhibitors detected in the total library. The total number of hits detected for each library were in agreement with the Venn diagram and also fulfilled our hit selection criteria of $\geq 75 \%$ inhibitory 
activity and growth inhibition in at least 2 out of 3 independent screens (at $20 \mu \mathrm{M}$ ) for designation of compounds as anti-mycobacterial.

\subsubsection{NIH Diversity Set}

The two anti-mycobacterial compounds identified from the NIH Diversity set, NSC145611 and NSC402959, were only identified using an M. bovis BCG model in the screens. These compounds demonstrated a significant growth inhibitory activity in dose-response assays against $M$. bovis BCG and upon subsequent validation experiment against $M$. tuberculosis H37Ra but not against M. smegmatis $\mathrm{mc}^{2} 155$ at the maximum concentration tested $(100 \mu \mathrm{M})$. The anti-M. tuberculosis activity of NSC145611 and NSC402959 was $6.25 \mu \mathrm{M}$ and $12.5 \mu \mathrm{M}$ (MIC), respectively (Table 4.2).
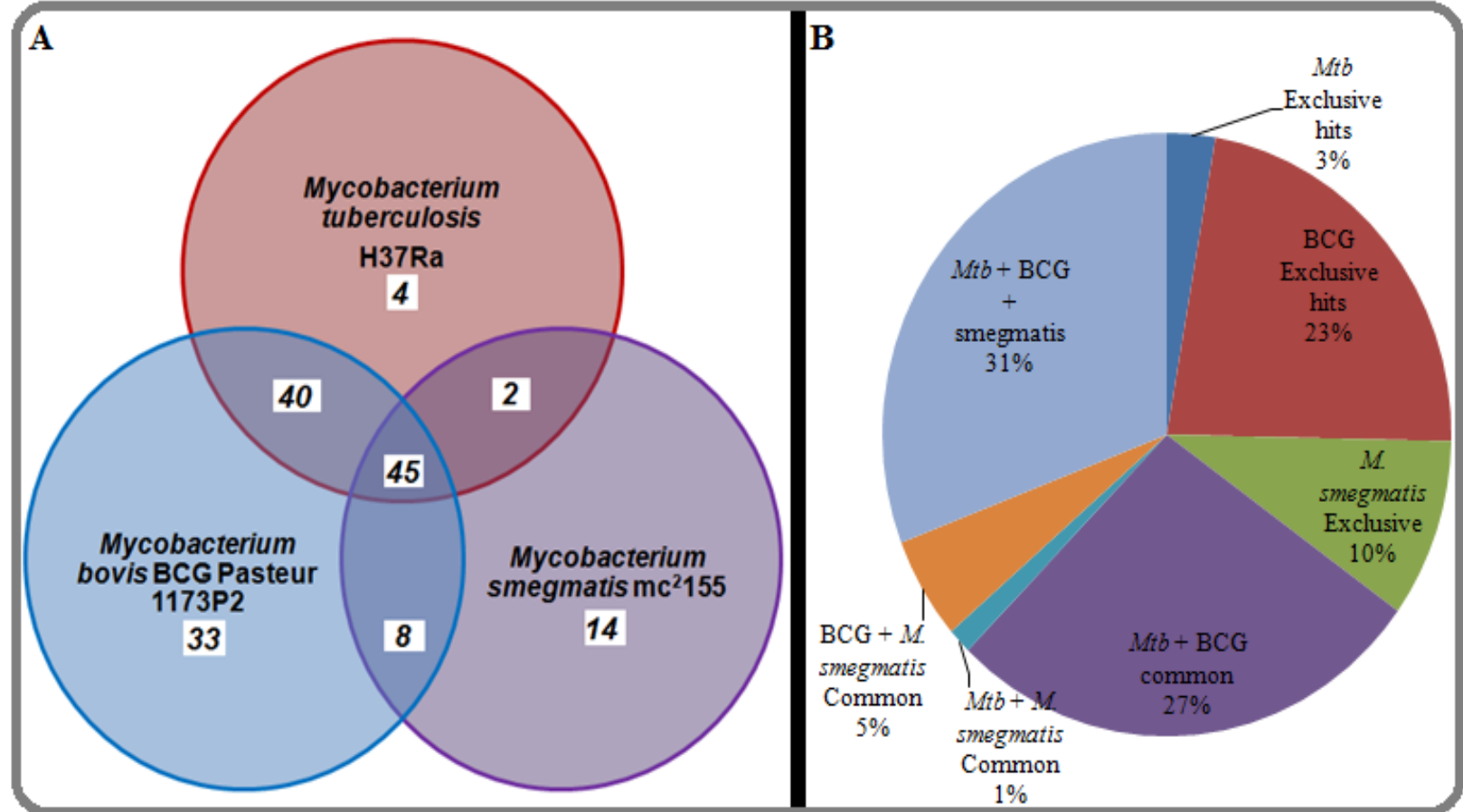

Figure 4.8: Venn diagrams for the NIH Diversity Set (A) Distribution of mycobacterial compounds detected in M. smegmatis $\mathrm{mc}^{2} 155$ (69 hits), M. bovis BCG Pasteur 1173P2 (126 hits) and M. tuberculosis H37Ra (91 hits) and (B) Percentage of inhibitors detected. Among the M. bovis BCG exclusive hits (26\%), most compounds also 
exhibited inhibitory activity against $M$. tuberculosis H37Ra in dose-response assays with only a slight difference in MIC values (data not shown).

\subsubsection{M. tuberculosis Specific Inhibitors}

Not all M. tuberculosis inhibitors were detected in the screens with M. bovis BCG. For example, NSC168467 was not detected in either of the screening models, yet exhibited a significant anti- $M$. tuberculosis activity upon validation experiments in $M$. tuberculosis H37Ra and therefore such compounds appeared to be M. tuberculosis specific hit in vitro. NSC168467 and other NIH compounds from the same series NSC168466 and NSC168468 are structural analogues of each other (PubChem database). Even though closely related in terms of their chemical structures (Figure 4.9), NSC168467 was not detected with M. bovis BCG (Table 4.2).

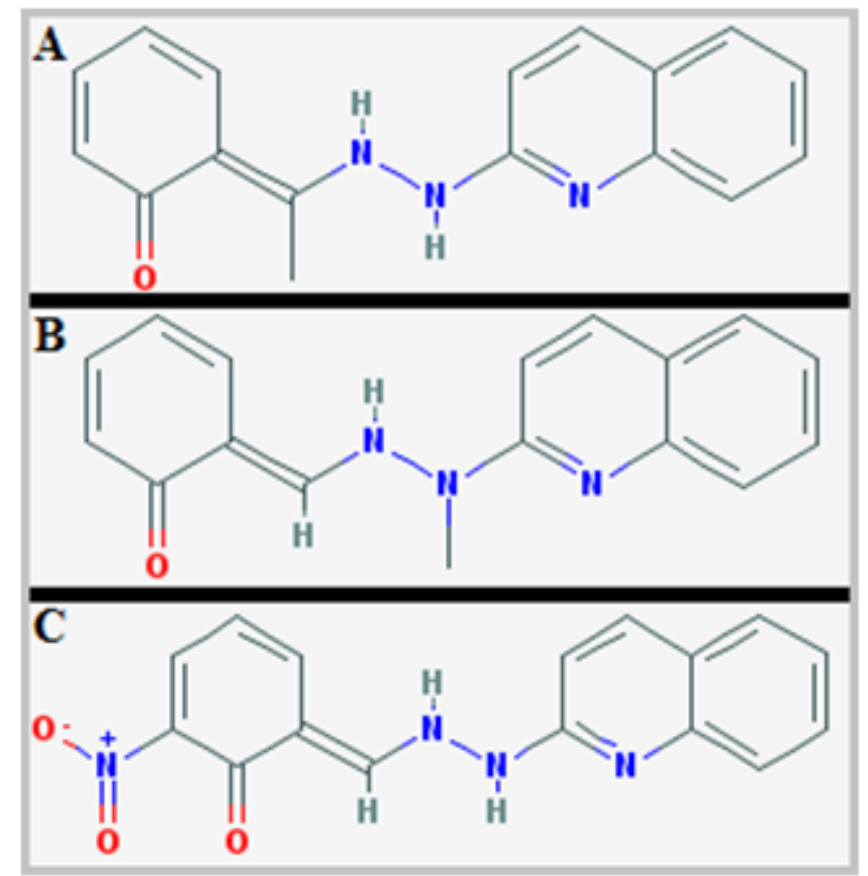

Figure 4.9: Chemical structures of anti-M. tuberculosis compounds (A) NSC168466, (B) NSC168467 and (C) NSC168468. MW for compounds A, B and C are $277.32 \mathrm{~g} / \mathrm{mol}, 277.3205 \mathrm{~g} / \mathrm{mol}$ and $308.29 \mathrm{~g} / \mathrm{mol}$, respectively (PubChem database). 
As discussed in Chapter 3, many compounds from the NIH library had anti-tumour or anticancerous activity, and despite their potent anti-mycobacterial activity, such compounds were not considered for further investigation due to known cytotoxic properties (section 3.3.1.1.4).

\subsubsection{False Positive Hits}

Some compounds were detected with $M$. bovis BCG in the screens but exhibited low antiBCG activity at the highest test concentration $(100 \mu \mathrm{M})$ in the validation studies. These include compounds NSC17061, NSC47151, NSC95570, NSC181486, NSC267461 and NSC306960. Since these compounds had no anti-mycobacterial activity in the validation studies, they were considered to be false-positives. Therefore, if such compounds were not found active in any of the three species upon validation in triplicate at the highest concentration tested $(100 \mu \mathrm{M})$, they were excluded from the list. 
Table 4.2: Validated compounds with anti-mycobacterial activity from the NIH library

\begin{tabular}{|c|c|c|c|}
\hline \multirow[t]{2}{*}{ NIH Compound } & \multicolumn{3}{|c|}{ MIC Values $(\mu \mathrm{M})$} \\
\hline & M. smegmatis & M. bovis $\mathrm{BCG}$ & M. tuberculosis \\
\hline NSC3907 & 25 & $\underline{12.5}$ & $\underline{3.125}$ \\
\hline NSC5159 & $\underline{1.56}$ & $\underline{1.56}$ & $\underline{1.56}$ \\
\hline NSC13480 & $\underline{12.5}$ & $\underline{12.5}$ & $\underline{12.5}$ \\
\hline NSC17061• & $\geq 100$ & $>\underline{100}$ & $>100$ \\
\hline NSC17600 & $\underline{25}$ & $\underline{25}$ & 25 \\
\hline NSC31660 & 50 & $\underline{25}$ & 12.5 \\
\hline NSC51001 & $\underline{12.5}$ & $\underline{12.5}$ & $\underline{12.5}$ \\
\hline NSC62709 & 25 & $\underline{50}$ & 12.5 \\
\hline NSC82892 & 50 & $\underline{25}$ & $\underline{12.5}$ \\
\hline NSC95570 • & $\geq 100$ & $>\underline{100}$ & $>100$ \\
\hline NSC125066 & $\underline{3.125}$ & $\underline{3.125}$ & $\underline{1.56}$ \\
\hline NSC145611 & $\geq 100$ & $\underline{50}$ & $\underline{6.25}$ \\
\hline NSC168466 & $>100$ & $>\underline{100}$ & $\underline{1.56}$ \\
\hline NSC168467 & $>100$ & $>100$ & $\underline{12.5}$ \\
\hline NSC168468 & $\geq 100$ & $>\underline{100}$ & $\underline{3.125}$ \\
\hline NSC189794 & $\underline{12.5}$ & $\underline{25}$ & $\underline{6.25}$ \\
\hline NSC267461 & $\geq 100$ & $\geq \underline{100}$ & $\underline{6.25}$ \\
\hline NSC268879 & $\underline{3.125}$ & $\underline{1.56}$ & $\underline{3.125}$ \\
\hline NSC285116 & $\underline{1.56}$ & $\underline{6.25}$ & $\underline{6.25}$ \\
\hline NSC305787 & 25 & $\underline{25}$ & $\underline{6.25}$ \\
\hline NSC306960 & $>100$ & $>\underline{100}$ & 12.5 \\
\hline NSC310551 & $\underline{6.25}$ & $\underline{12.5}$ & $\underline{6.25}$ \\
\hline NSC311153 & $\underline{25}$ & $\underline{6.25}$ & $\underline{6.25}$ \\
\hline NSC321206 & 25 & $\underline{25}$ & $\underline{3.125}$ \\
\hline NSC333003 & $\geq 100$ & $>\underline{100}$ & $\underline{1.56}$ \\
\hline NSC363182 & 50 & $\underline{25}$ & $\underline{3.125}$ \\
\hline NSC402959 & $>100$ & $\underline{25}$ & $\underline{12.5}$ \\
\hline NSC659779 & $\underline{3.125}$ & $\underline{0.781}$ & $\underline{3.125}$ \\
\hline
\end{tabular}

Underlined values indicate the species in which an inhibitor was detected during screening. All compounds which gave an MIC of at least $25 \mu \mathrm{M}$ in $M$. tuberculosis upon validation in dose-response experiments are listed. 
Dose-response assays provide better indication of any differences in the inhibitory profiles, compounds marked $(\bullet)$ were detected with $M$. bovis BCG but had no inhibitory activity against $M$. tuberculosis H37Ra in validation assays and were therefore considered as false-positives. All compounds were tested at concentrations up to 100 $\mu \mathrm{M}$.

\subsubsection{Z-factor for Screens}

Data received from HTS were statistically analysed to determine data integrity and fidelity of screens from z-factors values calculated (section 3.3.2). Two antibiotic controls were included in each plate for HTS, rifampicin and streptomycin. The mean z-factors for rifampicin were 0.86 (M. tuberculosis), 0.91 (M. bovis BCG) and 0.95 (M. smegmatis). The mean z-factors for streptomycin were $0.87,0.92$ and 0.96 for M. tuberculosis, M. bovis BCG and M. smegmatis, respectively. Values for all controls were within the dynamic range of a screening window coefficient as described by Zhang et al. [327] suggesting that the screening results obtained in this study were reliable.

\subsubsection{Genomic Comparison of the Mycobacteria}

The variation in chemical library inhibitory profiles was compared with the level of variation at the genomic level for the three mycobacterial species, M. smegmatis $\mathrm{mc}^{2} 155$, M. bovis BCG Pasteur 1173P2 and M. tuberculosis H37Ra. The Multi-Genome Homology tool (from J. Craig Venter Institute, available at http://cmr.jcvi.org/cgi-bin/CMR/CmrHomePage.cgi) was used to determine the level of similarity among the conserved orthologues between the three species. M. tuberculosis H37Ra was selected as the reference genome; whereas, M. bovis BCG Pasteur $1173 \mathrm{P} 2$ and M. smegmatis $\mathrm{mc}^{2} 155$ were selected as comparison genomes. A cut-off of at least $50 \%$ protein similarity was used to determine similarities and differences between the three mycobacterial species. The genome analysis revealed that $2816(69.8 \%)$ of the 4034 protein-coding genes of $M$. tuberculosis H37Ra has orthologues in both $M$. 
smegmatis and M. bovis BCG. In addition, 1083 of the $M$. tuberculosis genes (26.9\%) were also common to $M$. bovis BCG but are not present in $M$. smegmatis. Approximatley $50 \%$ of the $M$. smegmatis genome containing 3540 genes do not have conserved orthologues in $M$. tuberculosis $\mathrm{H} 37 \mathrm{Ra}$ compared to only 87 genes for $M$. bovis $\mathrm{BCG}$ that lacked orthologues in M. tuberculosis H37Ra (Figure 4.10).

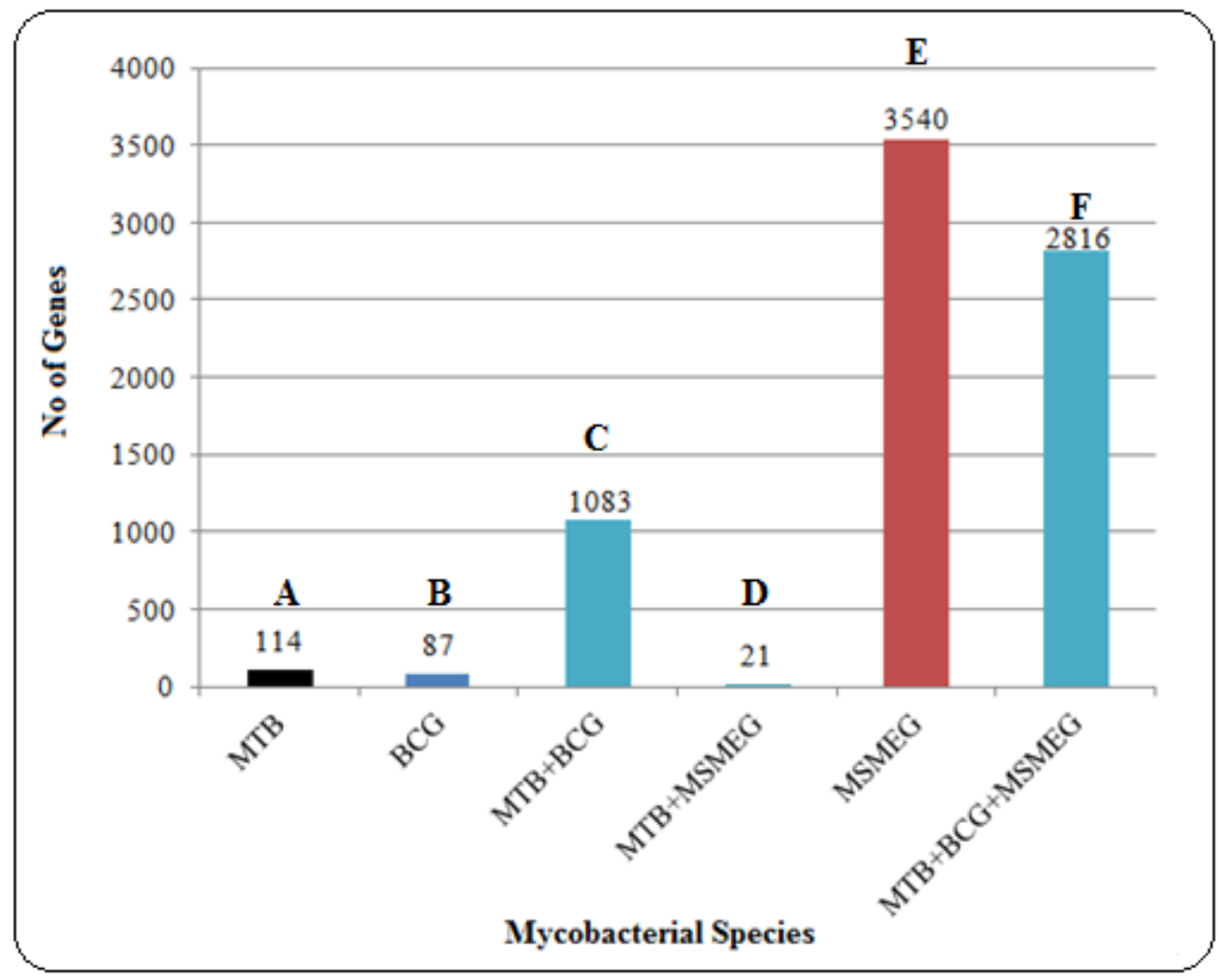

Figure 4.10: Comparison of the mycobacterial species for the analysis of conserved orthologues genes across M. tuberculosis H37Ra [MTB, (A)], M. bovis BCG Pasteur 1173P2 [BCG, (B)] and M. smegmatis mc $^{2} 155$ [MSMEG, (E)]. Reference proteins present in all comparison molecules and reference proteins not present in any of the comparison molecules are recorded. Using a 50\% cut-off for protein similarity, approximately 1000 M. tuberculosis genes were found to lack conserved orthologues in M. smegmatis in contrast to only $130 \mathrm{M}$. tuberculosis genes were absent in M. bovis BCG (A, B and E present total number of exclusive genes present in each species, whereas $\mathrm{C}, \mathrm{D}$ and $\mathrm{F}$ present conserved orthologues between $>1$ species). 


\subsubsection{Novel M. tuberculosis Inhibitor Identified from the NIH Library}

Of 2240 compounds screened for inhibitory activity from the NIH library, 60 compounds showed anti-M. tuberculosis activity at the $20 \mu \mathrm{M}$ test concentration. While some compounds were known anti-bacterials or anti-Tb agents, many inhibitors had no previously reported inhibitory activity. For example, NSC402959 (8-iodo-N-(8-iodo-10-phenyl-5H-phenazin-2yl)-10-phenylphenazin-2-imine) was identified from the NIH library screen as a potential inhibitor, as it showed good anti-mycobacterial activity and was non-cytotoxic to mammalian cells (Figures 3.12 and 4.13, respectively). Furthermore, it was also tested against a virulent in vitro model of $\mathrm{Tb}(M$. tuberculosis $\mathrm{H} 37 \mathrm{Rv})$ and retained decent activity with an MIC of $12.5 \mu \mathrm{M}$ (Figure 4.12).

\subsubsection{Dormicidal Activity of an RNase $H$ compound}

There could be different populations in old in vitro bacterial cultures which could escape from potential drug action [398]. It is therefore necessary to evaluate the sterilizing effect of inhibitors using in vitro models. The dormicidal effect of NSC402959 was also determined in stationary-cidal assays because the new growth inhibitors of MTBC are also expected to be active against persisters in order to complement the directly observed short course and to reduce the lengthy treatment time for $\mathrm{Tb}$. For the search of potential therapeutic agents that are active against non-replicating $M$. tuberculosis cells, Cole et al. have defined conditions for assay to identify such inhibitors [399]. Therefore, in order to examine if the effect of novel inhibitors (identified in this study) that are active against exponentially-growing cells, also retain similar activity after mycobacterial growth arrest; dormicidal assay was conducted (as described in section 2.7.3) with short-listed compounds against M. tuberculosis H37Ra (data not shown). NSC402959 showed bacteriostatic activity (rather than dormicidal) against M. tuberculosis H37Rv (Figure 7.9). Miller et al. have defined a range of culture/nutrient 
conditions for specifically selecting anti-mycobacterial compounds. Modifications in the culture conditions may have effect over respective inhibitory profiles of anti-mycobacterial compounds [252]. In this work, a carbon-limited condition in mycobacterial cultures was achieved by reducing the amount of glycerol by 10 fold (from $0.5 \%$ to $0.05 \% \mathrm{v} / \mathrm{v}$ ) or by removing glycerol completely (Figures 7.4, A-D), to examine effect on BCG growth and inhibitory activity of the test compounds. Figure 4.11 presents a dose-response curve in a nutrient-limited (C-) condition for M. tuberculosis H37Ra. There was no difference observed in anti-mycobacterial activity for NSC402959 during carbon starvation compared to nutrient rich culture conditions. The MIC for NSC402959 under both conditions against $M$. tuberculosis was in the $6.25 \mu \mathrm{M}$ to $12.5 \mu \mathrm{M}$ range (Figure 3.12 and Figure 7.7).

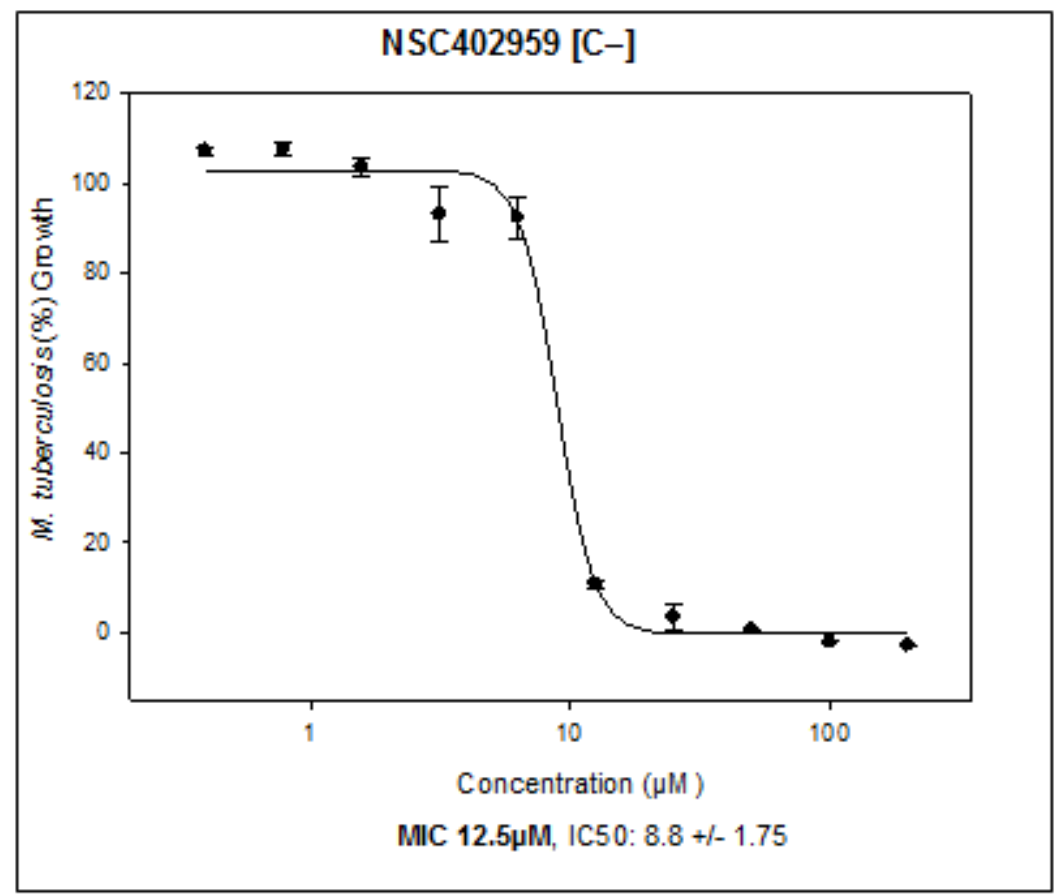

Figure 4.11: Anti-mycobacterial activity of NSC402959 against M. tuberculosis H37Ra under carbon starvation. Glycerol concentration for this experiment was $0.05 \%(\mathrm{v} / \mathrm{v}) 10$ times lower than the standard growth condition for the cultivation of slow-growing mycobacteria. 


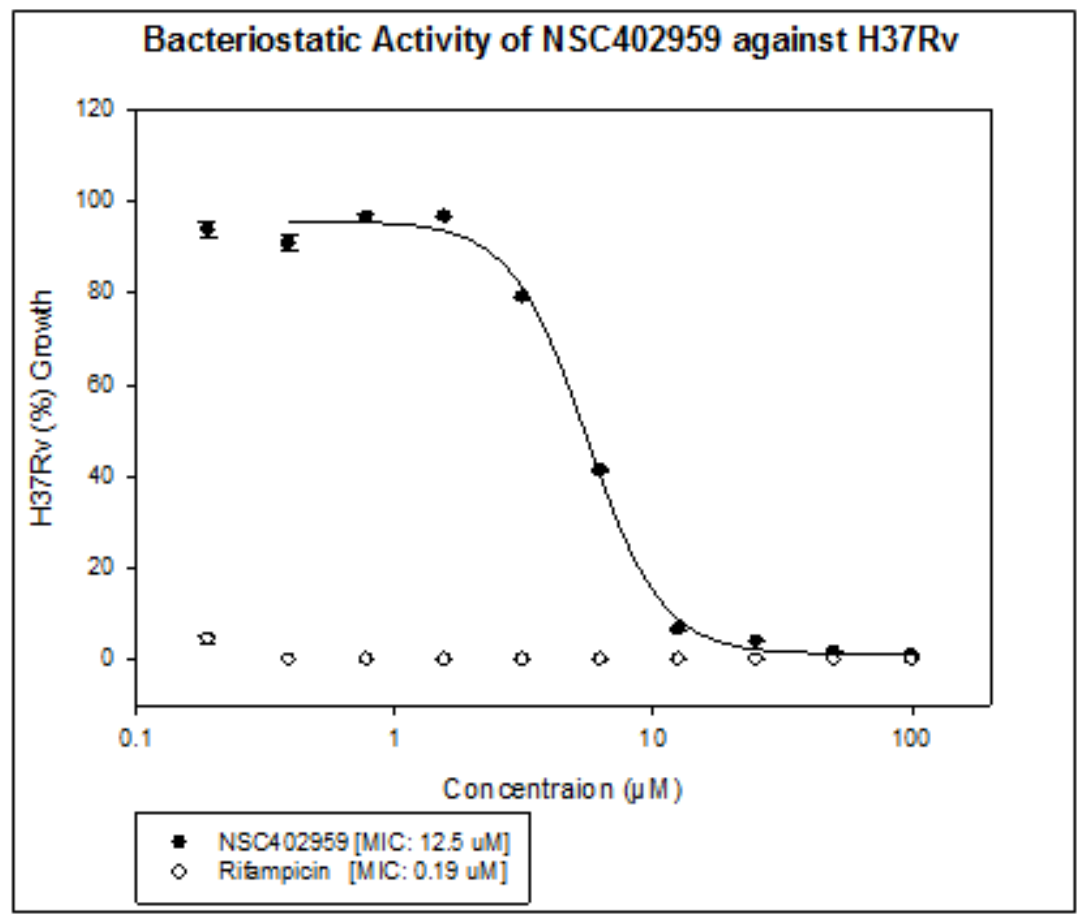

Figure 4.12: Growth inhibitory activity of NSC402959 and rifampicin in M. tuberculosis H37Rv. MICs against the virulent $M$. tuberculosis strain H37Rv for NSC402959 and rifampicin are $12.5 \mu \mathrm{M}$ and $0.19 \mu \mathrm{M}$, respectively. Studies were conducted under standard PC3 conditions at Johns Hopkins Centre for Tuberculosis Research, USA.

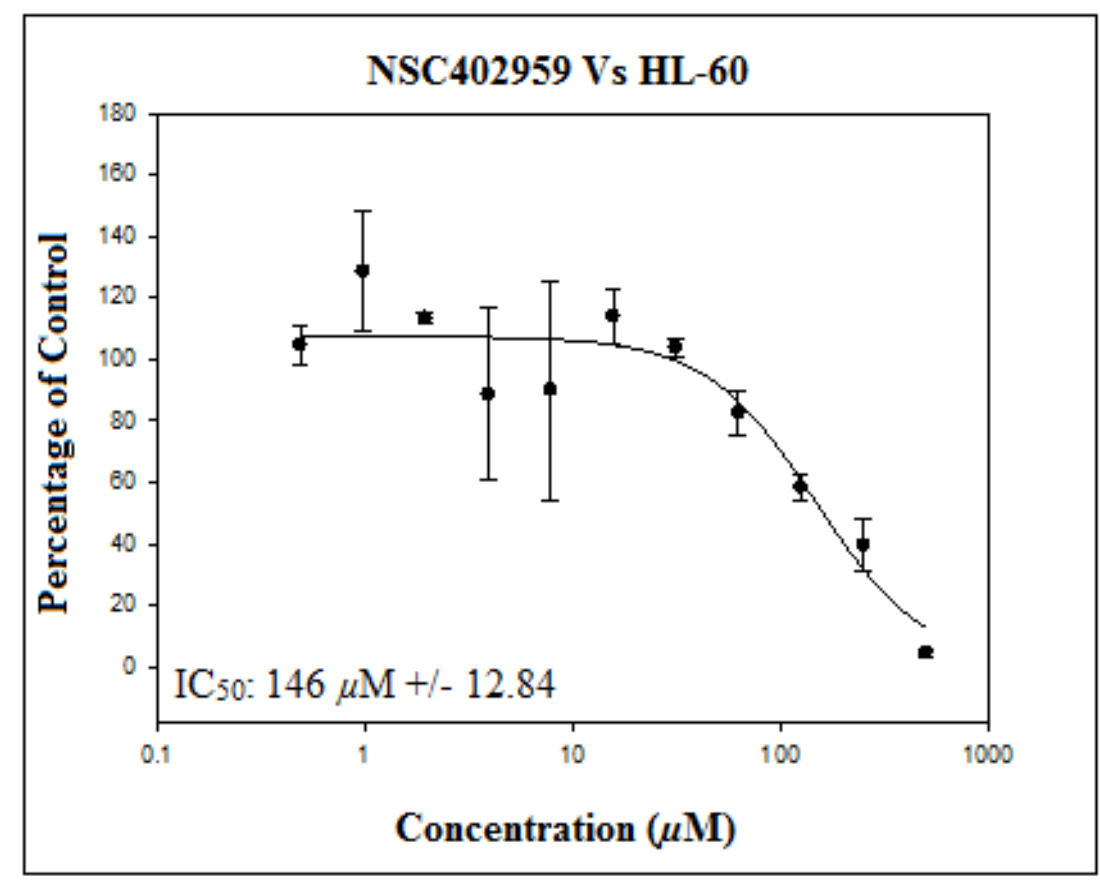

Figure 4.13: Cytotoxicity of NSC402959 against the HL-60 cell line. NSC402959 was not toxic to HL-60 cells yielding an $\mathrm{IC}_{50}$ of $146 \mu \mathrm{M}$, compared to rifampicin control $\left(\mathrm{IC}_{50}>400 \mu \mathrm{M}\right)$. 


\subsection{Activity Profile of Structural Analogues of NSC402959}

NSC402959 (8-iodo-N-(8-iodo-10-phenyl-5H-phenazin-2-yl)-10-phenylphenazin-2-imine) was identified as a new M. tuberculosis inhibitor in this study during HTS (Chapter 3). Based on its activity against mycobacteria, structural homologues of NSC402959 (Figure 4.14 A-B) were also imported from the NIH library to determine their efficacy. A preliminary structural analysis revealed that compounds NSC15381 (2-N,5-diphenyl-3-phenyliminophenazine-2,7diamine) and NSC603988 (N,5-dicyclohexyl-3-cyclohexyliminophenazin-2-amine) bear high structural similarity with NSC402959 and contain aromatic amide groups and radicals (Figure 4.14). While NSC15381 and NSC603988 were potent against M. tuberculosis in this study, unlike NSC402959, both of its analogues do not inhibit the activity of RNase H enzyme in HIV (PubChem database) and are toxic to mammalian cell line (Figure 3.14). In contrast, NSC402959 did not exhibit toxicity to any of the three cell lines tested (HL-60, Vero and J774.A1). NSC402959 is flanked by an iodine group which may play a role in it being less toxic to human cells (Figure 4.14-C). Both analogues and NSC402959 were also tested against a set of clinical strains of E. coli, S. aureus and $P$. aeruginosa to evaluate their activity profile. Only NSC402959 exhibited inhibitory activity against E. coli (Table 5.3); however, one of its analogues, NSC15381 has shown mild inhibitory activity against both $E$. coli and S. aureus (Figure 3.13). 


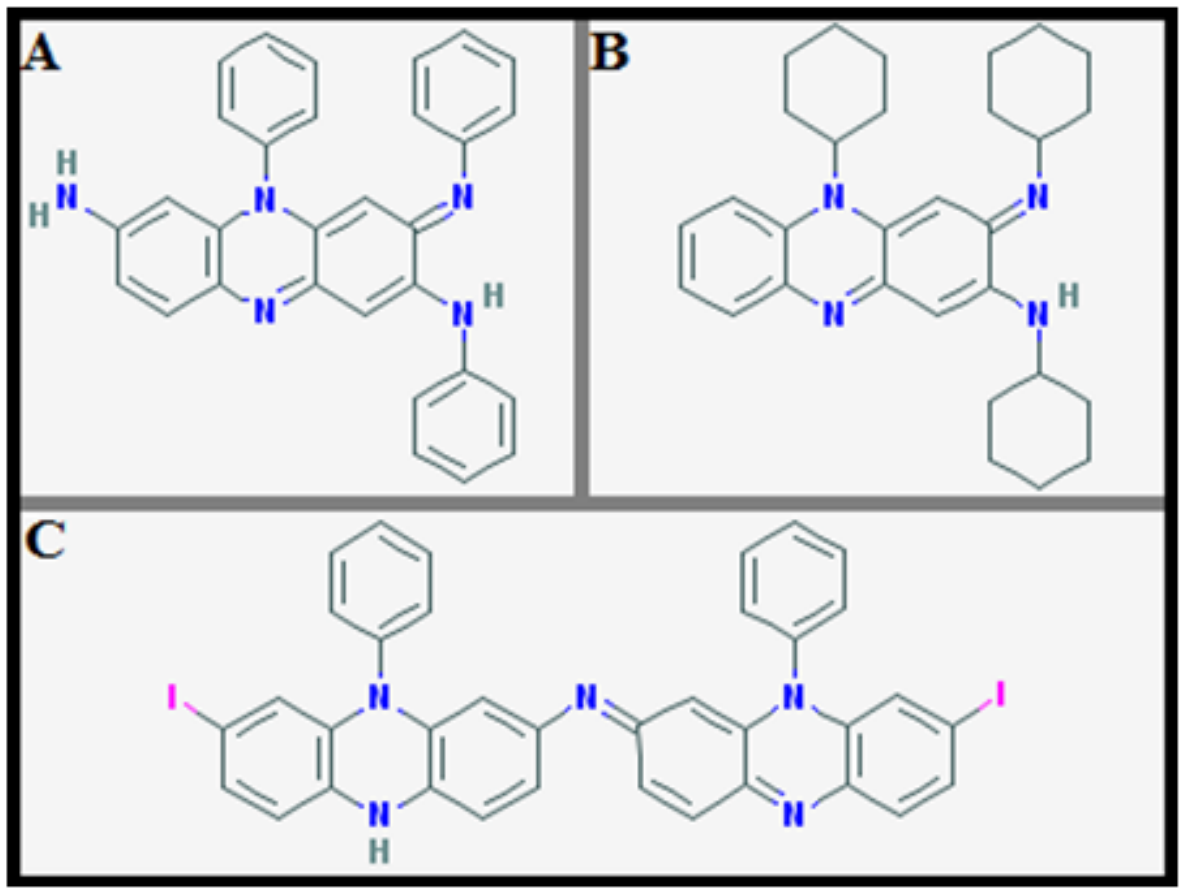

Figure 4.14: Chemical structures of (A) NSC15381, (B) NSC603988 and (C) NSC402959. MWs and MFs are (A) $453.54 \mathrm{~g} / \mathrm{mol}-\mathrm{C}_{30} \mathrm{H}_{23} \mathrm{~N}_{5}$, (B) $456.67 \mathrm{~g} / \mathrm{mol}-\mathrm{C}_{30} \mathrm{H}_{40} \mathrm{~N}_{4}$ and (C) $779.41 \mathrm{~g} / \mathrm{mol}-\mathrm{C}_{36} \mathrm{H}_{23} \mathrm{I}_{2} \mathrm{~N}_{5}$, respectively. Despite inhibitory activity of NSC402959 against human RNase H, it appears to be an interesting inhibitor and thus chemical derivatisation could be pursued to generate more effective analogues that are active against HIV and M. tuberculosis but do not target human RNase H. Chemical structures of the compounds were retrieved from the PubChem database.

The structures of NSC168466, NSC168467 and NSC168468 are very similar to one another (Figure 4.9), and so also were their anti-M. tuberculosis activity (Table 3.6). Likewise, NSC145611 and NSC145612 are high molecular weight compounds from the NIH library and have complex chemical structures but also appear very similar (Figure 4.15). Interestingly NSC145611 appeared to be highly active against M. tuberculosis H37Ra (MIC $6.25 \mu \mathrm{M})$ but its structural homologue NSC145612 did not exhibit a similar level of inhibitory activity and thus was not considered as the anti-mycobacterial of choice in this study (Table 3.6). 


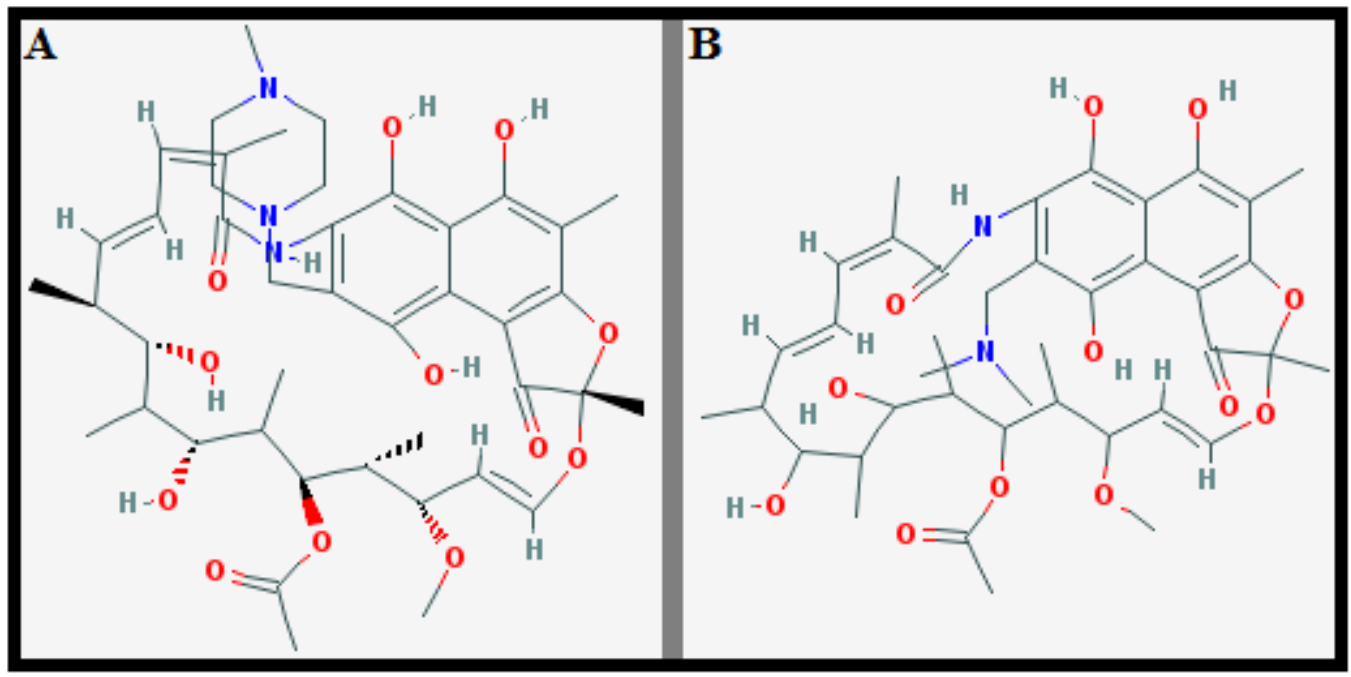

Figure 4.15: Chemical structures for anti-mycobacterial compounds. (A) NSC145611 and (B) NSC145612, MWs and MFs are $809.94 \mathrm{~g} / \mathrm{mol}-\mathrm{C}_{43} \mathrm{H}_{59} \mathrm{~N}_{3} \mathrm{O}_{12}$ and $754.86 \mathrm{~g} / \mathrm{mol}-\mathrm{C}_{40} \mathrm{H}_{54} \mathrm{~N}_{2} \mathrm{O}_{12}$, respectively. Structural information for the chemical compounds NSC145611, NSC145612 and other adjacent compounds in the NIH series were retrieved from the PubChem database.

NSC145611 has an extra nitrogen atom (Figure 4.15-A) which may play a role in its enhanced anti-mycobacterial activity (MIC $6.25 \mu \mathrm{M}$ ) compared to its analogue NSC145612 (MIC $50 \mu \mathrm{M}$ ), which has only 2 nitrogen atoms. NSC145611 in particular is closely related to a known anti-tubercular, rifamycin (PubChem database). Nitric-oxide (NO) has previously been shown to have potent anti-microbial effects [400], and the findings above are in agreement with previous reports for the antimicrobial effect of NO [401], such as its activity against mycobacteria [402]. This may explain the better efficacy of chemical entities with extra nitrogen and oxygen groups. However, Thomas et al. have suggested that the enhanced anti-mycobacterial effect of these compounds is associated with the presence of the phenylN1 group on the side chains rather than their increased lipophilicity [403-404]. The inhibitory activity of such compounds against $M$. tuberculosis could be attributed to their intrinsic antibacterial property rather than the lipophilic nature of these compounds. 


\subsection{Discussion}

Understanding the genetic variability and virulence of the disease-causing mycobacterial strains is important to select a suitable model for screening drugs and unknown inhibitors. In this study, the sensitivities of M. smegmatis and M. bovis BCG were evaluated as surrogate in vitro models for the detection of anti-M. tuberculosis compounds.

M. smegmatis has long been regarded as an environmental saprophyte of no clinical significance. A number of reports have described clinical effects of $M$. smegmatis infection [405-407]. For example, M. smegmatis has been associated with pleuro-pulmonary disease [408]. A review published in 2002, described a range of community-acquired and healthcare-associated diseases that were attributed to M. smegmatis including cellulitis, soft tissue necrosis, lipoid pneumonia and catheter sepsis [384]. While it is apparent that it may not be possible to strictly define $M$. smegmatis as a non-pathogenic species of mycobacteria, number of known clinical cases caused by $M$. smegmatis is extremely low relative to nearly nine million new cases of $\mathrm{Tb}$ occurring each year. M. smegmatis has been employed in $\mathrm{Tb}$ research for a range of studies because it offers several advantages over other Tb models e.g. fast growth, no special nutrient requirements and minimum risk to health workers. $M$. smegmatis on its own presents many similarities to the slow-growing pathogenic species of $\mathrm{Tb}, \mathrm{H} 37 \mathrm{Rv}$. For instance, the hypoxia response of $M$. smegmatis mimics the response in $M$. tuberculosis [124, 409-411]. Pathogenic and non-pathogenic species can also be made more comparable, if genetically manipulated [412]. However, the relevance of its use with respect to $\mathrm{Tb}$ drug discovery is questionable. The $M$. smegmatis controversy with respect to its use and whether it represents a suitable model for studying the pathogenicity of M. tuberculosis was initially raised in 2001 [391] (section 4.3.2). The issue of its reliability in terms of detecting M. tuberculosis inhibitors, however, has not been previously addressed. Since it is important to select the most appropriate model for the discovery of new agents that could be 
developed into drugs for the treatment of multi-drug resistant and latent forms of the disease. This study was conducted to directly measure the ability of $M$. smegmatis HTS to detect antiM. tuberculosis compounds. Three mycobacterial species were used to screen the compound libraries, and the data produced from the screening were compared for each Tb model with regard to similarities and variations in anti-mycobacterial hits relative to the reference strain, M. tuberculosis H37Ra. M. tuberculosis H37Ra was selected as a representative model organism of M. tuberculosis in this work since a good correlation between the H37Ra and H37Rv has been previously reported with regard to drug susceptibility [352].

An initial data analysis from the library screening in this study revealed that $48-58 \%$ of $M$. tuberculosis inhibitors could not be identified when $M$. smegmatis was used, representing approximately only a $50 \%$ sensitivity for detecting $M$. tuberculosis inhibitors in the library screens. However, with $M$. bovis BCG employed as an in vitro model for drug screening in parallel screens performed under identical growth conditions, a substantially higher percentage of $M$. tuberculosis inhibitors were detected, $66 \%$ and $93 \%$ for LOPAC and the NIH chemical libraries, respectively (section 4.4).

These data suggest that $M$. bovis BCG is more effective in vitro model than $M$. smegmatis for the detection of $M$. tuberculosis inhibitors. An example of a compound that was identified by using $M$. bovis BCG but not detected with $M$. smegmatis includes ammonium pyrrolidine dithiocarbamate (APDC). APDC has previously been reported to be an active inhibitor of $M$. tuberculosis $\mathrm{H} 37 \mathrm{Rv}$ with an MIC of $0.125 \mu \mathrm{g} / \mathrm{mL}(0.761 \mu \mathrm{M})$ [290]. APDC has also been found to be active against persistent cells with a minimum bactericidal concentration (MBC) as low as $0.5 \mu \mathrm{g} / \mathrm{mL}$ [413]. Similarly, another M. tuberculosis inhibitor tetraethyl thiuram disulphide (Disulfiram) was also detected from the LOPAC library with M. bovis BCG but not with M. smegmatis. Disulfiram has previously been used for the treatment of alcoholism 
in $\mathrm{Tb}$ patients [301]. Other examples include 2-chloroadenosine and niclosamide (Table 4.1 and section 3.3.1.1.3).

From these findings in the present study, it is evident that some of the potential $M$. tuberculosis inhibitors were not detected with $M$. smegmatis screening, although the inhibitors were active against both avirulent and virulent Tb strains, M. tuberculosis H37Ra and M. tuberculosis $\mathrm{H} 37 \mathrm{Rv}$ (Figures 3.12 and 4.12, respectively). For example compounds from the NIH library, NSC333003 and NSC402959 (section 4.4.9) were not detected with $M$. smegmatis but were identified by $M$. bovis BCG in the screens. Both compounds were also tested against the virulent Tb strain, M. tuberculosis H37Rv and were found to exhibit good anti-mycobacterial activity. NSC402959 in particular appeared to be bacteriostatic in its activity against $M$. tuberculosis H37Rv (Figure 7.9) and did not exhibit cytotoxicity towards a number of mammalian cell lines (Table 2.3) HL-60 (Figure 4.13), Vero (Figure 7.6) and J774 (Figure 7.8), (section 4.4.9.1). NSC402959 had been previously shown to have an antiE. coli RNase HI activity (PubChem database). Ribonuclease H1 (RNase HI) is an endonuclease responsible for cleaving the RNA strand of RNA-DNA hybrids [414]. This RNA-DNA cleavage is an essential cellular activity required for nucleic acid replication and repair in prokaryotic and eukaryotic species [415-419]. In M. tuberculosis, RNase HI and RNase HII activities are encoded by the $r n h \mathrm{~A}$ and $r n h \mathrm{~B}$ genes (ORFs Rv2228c and Rv2902c, respectively) [420]. We hypothesized that NSC402959 may have RNase HI as a primary protein target in M. tuberculosis. To determine this, further work was carried out to examine if RNase HI inhibitors possess specificity for Rv2228c, which is described in Chapter 5.

While few inhibitors that were active against $M$. tuberculosis could not be detected with $M$. smegmatis, many hits identified with this species in HTS were also validated in $M$. tuberculosis (Tables 4.1 and 4.2). Moreover, a new anti-Tb drug, TMC207 had previously been identified during HTS using M. smegmatis [421]. This demonstrates that it could still be 
useful in $\mathrm{Tb}$ research. The data obtained in the present study however indicate a lower sensitivity and ability of this model to detect positive hits compared to $M$. bovis BCG. The reason for differential inhibitory responses of different drugs in these mycobacterial species is not known, but is likely to be the fact that $30 \%$ of $M$. tuberculosis genes lack conserved orthologues in M. smegmatis. The higher genetic similarity of $M$. bovis BCG with $M$. tuberculosis along with its non-pathogenic nature makes it a more attractive model for anti$\mathrm{Tb}$ drug discovery. However, the slow growth rate of this species (two weeks are required for bacteriostatic drug screening for M. bovis BCG compared to four days for M. smegmatis) needs to be taken into account for its use in drug screening.

\subsection{Conclusion}

The research undertaken in this chapter highlights the importance of selecting an appropriate model for the screening M. tuberculosis inhibitors in vitro. Data produced in this study lend support to $M$. bovis BCG as more sensitive and reliable model than $M$. smegmatis to detect $M$. tuberculosis inhibitors. 
Chapter 5

RNase HI as a Target for Dual Inhibitors of $\mathbf{T b}$ and HIV 


\subsection{Introduction}

In Chapter 4, a new mycobacterial inhibitor was described, NSC402959. This compound has been shown to inhibit RNase $\mathrm{H}$ from a number of pathogens including HIV (PubChem database). We reasoned that NSC402959, and other RNase H inhibitors could potentially function as dual inhibitors of both $M$. tuberculosis and the Human Immunodeficiency Virus (HIV).

\subsubsection{Tb and HIV - the Two Pandemics}

HIV is a retrovirus that impairs the function of immune system and is responsible for AIDS (Acquired Immunodeficiency Syndrome) in humans [422-424]. HIV primarily targets the host immune system and makes it weak and incompetent, which further jeopardizes patient's health by making them vulnerable to a number of other opportunistic infections, such as $\mathrm{Tb}$ [425-426]. With the emergence of the HIV pandemic, there has been a resurgence of $\mathrm{Tb}$ [427]. Tb is the most common HIV-related opportunistic infection [428-430], and HIV has been considered as the inducing factor for the development of $M$. tuberculosis infection into an active disease due to malfunctioning of the immune system [431]. Moreover, reactivation of latent M. tuberculosis is quite common among HIV positive patients [432].

\subsubsection{HIV/Tb - A Lethal Combination}

M. tuberculosis and HIV co-infection is a lethal combination since both infections fuel each other, [433]; therefore the death rate is very high among such individuals due to synergy between these two infections [434-435]. HIV targets CD4 immune cells (T lymphocytes) which are required to activate macrophages in $M$. tuberculosis infections in order to halt their active replication. Therefore, patients with an ongoing HIV infection are highly vulnerable to 
catch an M. tuberculosis infection [436], especially in the resource-scarce communities where the $\mathrm{Tb}$ incidence rate is already high.

\subsubsection{Global Facts about HIV/Tb Syndemic}

Highly Active Anti-retroviral Therapy (HAART) became available in 1995 with a positive health impact for HIV-infected individuals [437]. AIDS patients lived longer [438-439], and the number of HIV cases started to fall after the advent of HAART [440]. Despite this new approach to disease control, 5.8 million new cases of HIV were still reported in 1997 along with 2.3 million AIDS-related deaths [441]. However, with the continuous rise in annual $\mathrm{Tb}$ incidence worldwide (Figure 1.4), the situation became even worse than it was before, indicating a global HIV epidemic [442]. A total of 39.5 million people were estimated to be infected with HIV including those newly-infected (4.3 million) from a deadly viral infection which claimed 2.9 million lives in 2006 [443]. In 2010 alone, an estimated 2.7 million new HIV cases along with approximately 1.8 million deaths were reported [444]. Despite the availability of HAART, the number of people living with HIV was still 34 million in 2010 [445]. The HIV epidemic therefore poses serious risks for worldwide Tb control management [446]. Tb currently claims a life every 20 seconds (about two million lives each year) [447]. Current $\mathrm{Tb}$ infection can accelerate the onset of HIV infection [448], highlighting the importance of new drug treatments that have a combined action against these two pandemics.

\subsection{Demand for Amendments to the Current HIV Therapeutic Regimen}

The 2010 WHO global report indicated that most countries would not be able to achieve the Millennium Development Goal (MDGs) No. 6 of halting and reversing the spread of HIV transmission [445]. Since both Tb and HIV cause high mortalities and there are antagonistic effects of anti-retroviral agents on current anti-Tb chemotherapy [449], it is important to 
search for more potent and compatible drugs that not only contain and reduce HIV infections but are also effective against co-infecting Tb infections.

Some of the problems associated with both anti-mycobacterial and anti-viral therapies are presented in sections 5.2.1 and 5.2.2, respectively.

\subsubsection{Current Anti-Tb Therapy and Associated Problems}

(1) Multi-drug regimen, a high daily pill burden (section 1.8.2 and Table 7.1).

2 Prolonged treatment - relapse issue (Figure 1.2).

3 Adverse effects of the Tb treatment regimen [167, 186-188, 450-451].

4 Drug-resistant $\mathrm{Tb}$ does not respond to currently-available anti-Tb drugs (section 1.8.3.4).

\subsubsection{Current Anti-HIV Therapy and Associated Problems}

(1) Only non-nucleoside reverse transcriptase (RT) inhibitors introduced in 1997 are available for the successful suppression of viral replication e.g. Nevirapine (NVP), Delavirdine (DLV) and Efavirenz (EFV), all approved by the US Food and Drug Administration (FDA).

2 Antagonistic effect of anti-Tb drugs with antiretroviral therapy. Some drugs that lead to toxicities are contra-indicated.

3 Adverse effects of available antiretroviral drugs [452-453].

4 Development of drug resistance and lack of effective vaccine production.

\subsubsection{Mutual Detrimental Effect of Bacterial and Viral Infections}

( Adverse effects of HAART therapy in TB co-infections is common [454].

2 Mortality rates are higher in HIV/Tb co-infected individuals, if left untreated [455]. 


\subsubsection{Possible Solution - A Dual Therapy}

It is apparent that drug resistance and regimen incompatibility between two therapies is the common problem associated with treatment of Tb/HIV infections. This could possibly be simplified by targeting something that is essential for both the bacteria and the virus (section

5.3.2). RNase $\mathrm{H}$ is a class of enzymes that non-specifically degrades the RNA strand of RNA-DNA hybrids [414]. There is a diverse range of RNase $\mathrm{H}$ found in both prokaryotes [456] as well as eukaryotes [419]. RNase $\mathrm{H}$ activity was not described in M. tuberculosis until recently [420]. RNase $\mathrm{H}$ is present in both species and appears to be essential for their life cycles [457]. HIV RNase HI has approximately $31 \%$ identity at protein level with RNase HI from M. tuberculosis [420, 458]. This enzyme may present a potential target in both HIV and M. tuberculosis (section 5.6). The current literature has very few reports on RNase $\mathrm{H}$ as a novel drug target in HIV and few inhibitors of RNase $\mathrm{H}$ activity have been identified [459463]. No anti-HIV RT-RNase H specific drugs are available for therapy nor has this class of enzymes been investigated for their therapeutic potential for the treatment of $\mathrm{Tb}$. Given that RNase $\mathrm{H}$ is a common drug target in HIV/Tb, co-inhibition of these two diseases could be possible. We hypothesise common inhibitors that specifically target RNase HI can be identified and developed. Therefore, RNase $\mathrm{H}$ could potentially serve as a new target in $M$. tuberculosis as well as cause simultaneous inhibition of HIV replication. New RNase H specific inhibitors, with different mechanism of action to drugs currently in use, would be expected to also have activity against drug-resistant strains, which would be crucial in containing these infections. 


\subsubsection{Aims for this Study}

1. To develop a repertoire of RNase H inhibitors of HIV-I and HIV-II.

2. To test anti-HIV RNase $\mathrm{H}$ compounds for their anti-mycobacterial activity in vitro against M. tuberculosis H37Ra in growth inhibition assays.

3. To identify a single-target dual inhibitor of M. tuberculosis and HIV that does not interfere with human RNase H enzymes.

4. To develop a biochemical assay that specifically detects anti-RNase HI activity of our active compounds against the purified proteins (Rv2228c and Rv2902c) from $M$. tuberculosis.

\subsection{Dual Inhibitors of M. tuberculosis and HIV}

\subsubsection{Definition of a Dual Inhibitor}

A dual inhibitor is a chemical compound and/or natural product that has a common target (e.g. RNase H) in two separate biological species (e.g. bacterial and viral) and shows a consistent inhibitory activity against such species in in vitro replicative assays. These compounds hold the potential to achieve co-inhibition of the two important human infections Tb and HIV.

\subsubsection{Novelty in Approach}

RNase $\mathrm{H}$ has recently been investigated for its potential to be a valid drug target for HIV but RNase $\mathrm{H}$ has never been studied in M. tuberculosis in this regard (section 5.3.2). Therefore the identification of single-target (RNase H) inhibitor in $M$. tuberculosis is the first step in the development of a dual therapy for both diseases, thus making this study unique. 


\subsubsection{RNase $H$ as a Viable Target in HIV and M. tuberculosis}

The genome of HIV $(9.8 \mathrm{~kb})$ encodes only 9 genes consisting of two structural and six regulatory genes that encode approximately 15 proteins including an important enzyme reverse transcriptase (RT) [464]. HIV-RT also possesses an RNase HI activity [465]. Recent studies to explore novel drug targets in HIV have shown that RNase $\mathrm{H}$ represents a viable drug target and is essential for the life cycle of HIV [466-469]. However, little is known about the specific binding of RNase $\mathrm{H}$ inhibitors to their active sites on the enzyme. In some of the studies that are available, it has been suggested that there might be multiple binding sites for various RNase $\mathrm{H}$ inhibitors [460, 470].

Cole et al. has sequenced the genome of $M$. tuberculosis H37Rv, and this has helped scientists make significant advancements in the field of Tb biology [17]. However, the function of the open reading frames (ORFs) of H37Rv has not been completely determined. Watkins et al. have recently demonstrated associated RNase $\mathrm{H}$ (type I) activity in $M$. tuberculosis [420]. The genome of M. tuberculosis (section 4.3.1) contains the rnhA gene (ORF Rv2228c) and $r n h \mathrm{~B}$ gene (ORF Rv2902) that have been functionally determined to possess RNase HI and RNase HII activities, respectively. RNase HI (Rv2228c) is a multifunctional protein and also seems to have associated cobC and acid phosphatase activity [420]. RNase HI activity appears to be essential for all prokaryotes [471], and can be used in scaling up the inhibitory activity of anti-RNase $\mathrm{H}$ compounds. Given specific activity of inhibitors (such as NSC112200, Figure 7.14) identified in this study, RNase HI could be a viable drug target in M. tuberculosis (section 5.4.3). 


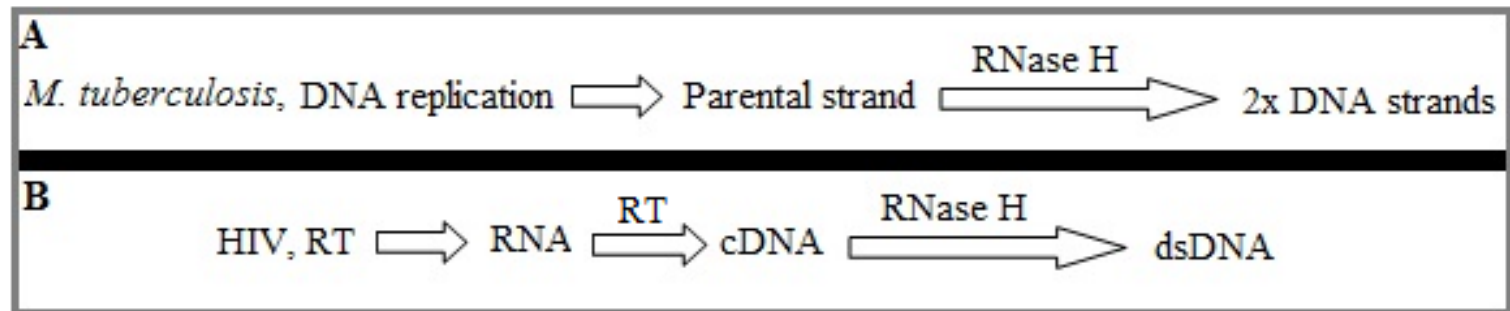

Figure 5.1: RNase H activity required for DNA replication in (A) M. tuberculosis and (B) HIV, RT. RNase $\mathrm{H}$ activity is essential for the replication of DNA strands in both species because of its cleavage of the RNA strand of RNA-DNA hybrids. RNase $\mathrm{H}$ in HIV is a bifunctional enzyme having the RT activity for making cDNA from RNA (DNA polymerase) as well as an RNase $\mathrm{H}$ activity for nicking the RNA strand of an RNADNA hybrid. RT, reverse transcriptase enzyme.

\subsubsection{Biochemical Assay to Detect RNase H Activity}

RNase $\mathrm{H}$ enzyme is responsible for cleaving the RNA strand of RNA-DNA hybrids before the DNA replication step (Figure 5.1). The specific activity of the RNase HI inhibitors identified in this study was measured using a biochemical assay as previously described [472].

\subsubsection{Principle of an RNase H Assay}

To measure the specific activity of RNase $\mathrm{H}$ enzyme and to detect the specific inhibitory activity of their inhibitors, DNA and RNA strands are labelled with dabcyl and fluorescein, respectively. When RNase $\mathrm{H}$ enzyme cleaves the RNA strand off the DNA strand, it produces fluorescence that can be detected by a spectrophotometer. However, in the presence of an inhibitor, the activity of RNase H enzyme is blocked and therefore no detectable fluorescence indicates the specific activity of an RNase H inhibitor (Figure 5.2). 


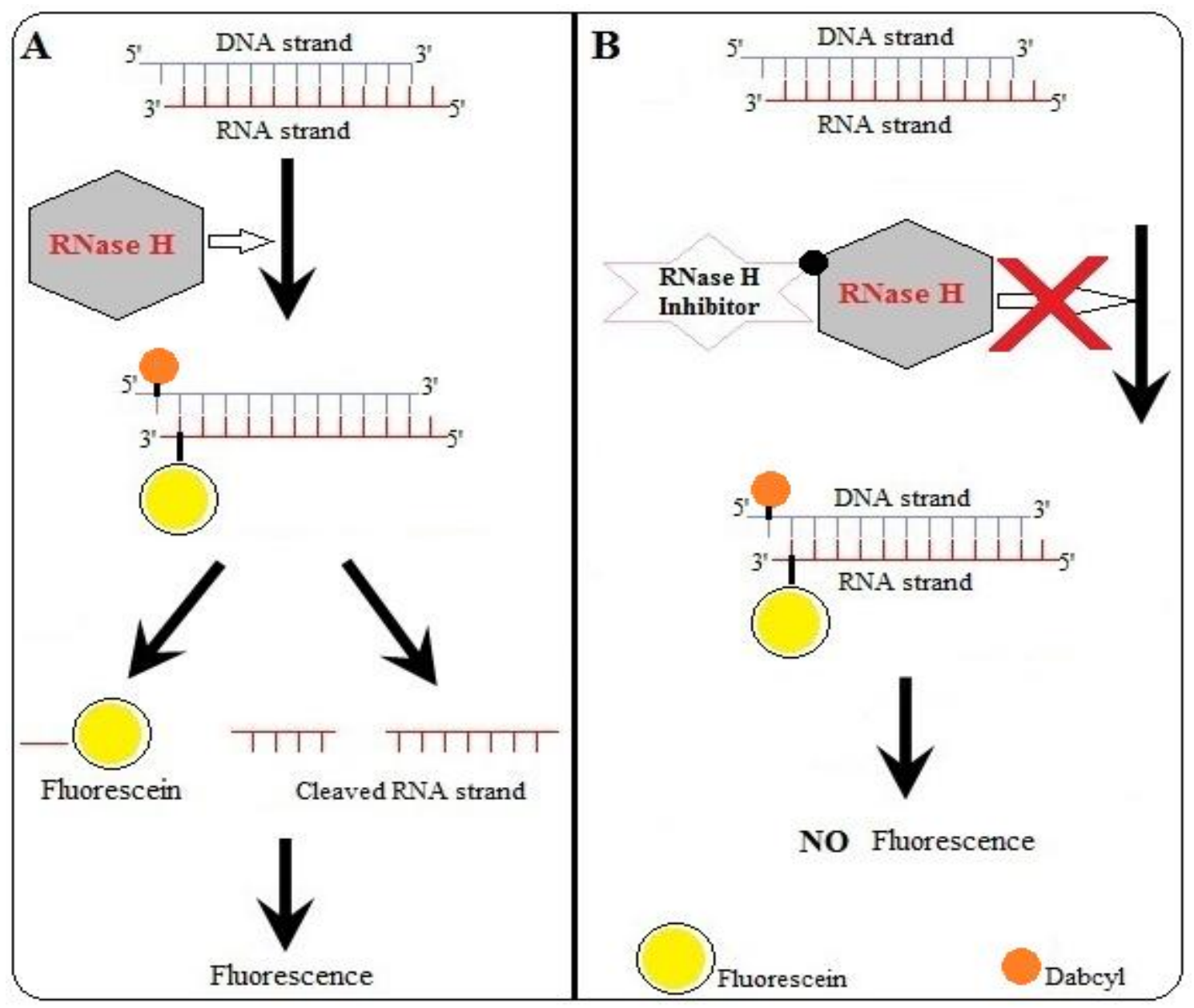

Figure 5.2: Principle of the RNase $\mathbf{H}$ assay. DNA and RNA strands are labelled with dabcyl (quencher) and fluorescein, respectively (Sigma Aldrich) (A) Shows no inhibition of the enzyme and therefore, an RNase H enzyme cleaves the substrate at the 3' end of an RNA strand and produces detectable fluorescence monitored by a spectrophotometer and (B) Shows enzyme inhibition by an RNase H inhibitor. The inhibitor blocks the activity of RNase H enzyme; therefore, it does not cleave the RNA strand thereby producing no detectable fluorescence in the presence of a dabcyl quencher. 


\subsubsection{Criteria for the Selection of Dual Inhibitors of Tb and HIV}

Compounds were selected on the basis of their inhibitory activity if;

1. They are known inhibitors of an HIV RNase H.

2. They inhibit $M$. tuberculosis growth in vitro with an $\mathrm{IC}_{50}$ of $<25 \mu \mathrm{M}$ in this study.

3. They are not cytotoxic towards the mammalian cell lines, HL-60 and J774.A1 tested.

4. They are active against $M$. tuberculosis $\mathrm{RNase} \mathrm{H}$ in an enzyme inhibition assay.

5. They do not inhibit human RNase H.

\subsection{Results}

\subsubsection{Anti-HIV RNase H Compounds}

All HIV RNase $\mathrm{H}$ inhibitors were selected from the PubChem database [www.ncbi.nlm.nih.gov/pccompound] and imported from the NIH to expand the repertoire of our RNase $\mathrm{H}$ inhibitors (section 2.6.7). Compounds, which gave an $\mathrm{IC}_{50}$ of $25 \mu \mathrm{M}$ or less were selected for further analysis and are listed in table 5.1.

The following work was completed to evaluate the anti-mycobacterial potential of the HIV RNase H inhibitors.

1. In vitro whole cell-based activity of anti-HIV RNase $\mathrm{H}$ compounds was monitored against M. tuberculosis $\mathrm{H} 37 \mathrm{Ra}$ in dose-response experiments.

2. All compounds were also tested against a number of other clinical strains (Table 5.3) to determine the spectrum of inhibitory activity based on their inhibition of $M$. tuberculosis H37Ra growth in vitro.

3. Compounds were then prioritised on the basis of their growth inhibitory potential and were further tested to evaluate their dormicidal activity against M. tuberculosis 
H37Ra (performed on over four week old cultures). Selected compounds were also tested for any shift in their inhibitory activity in nutrient-limited culture conditions (C).

4. Cytotoxicity assessment was done using HL-60 human cells to evaluate toxic effect of the inhibitors.

5. New RNase HI inhibitors of M. tuberculosis were identified that have previously shown activity against the HIV-RNase H enzymes.

6. Structural analysis was performed on the compounds identified in this study for comparison with the known inhibitors and current anti-HIV and anti-Tb drugs. Any previously-known biological activity of the inhibitors was also noted.

\subsubsection{In vitro activity of HIV RNase $H$ inhibitors against $M$. tuberculosis}

All anti-HIV RNase H compounds were tested against $M$. tuberculosis in a dose-response assay as described in section 2.7.2, and their $\mathrm{IC}_{50}$ values are tabulated below (Table 5.1). These inhibitors were tested at concentrations up to a maximum of $100 \mu \mathrm{M}$ and were ranked according to the level of inhibitory activity against $M$. tuberculosis. Compounds that exhibited mild-to-no inhibitory activity against $M$. tuberculosis $\mathrm{H} 37 \mathrm{Ra}$ (with an $\mathrm{IC}_{50}$ value of $>25 \mu \mathrm{M}$ ) were considered inactive and were excluded from the list (section 5.3.4). 
Table 5.1: Activity profile of RNase HI inhibitors

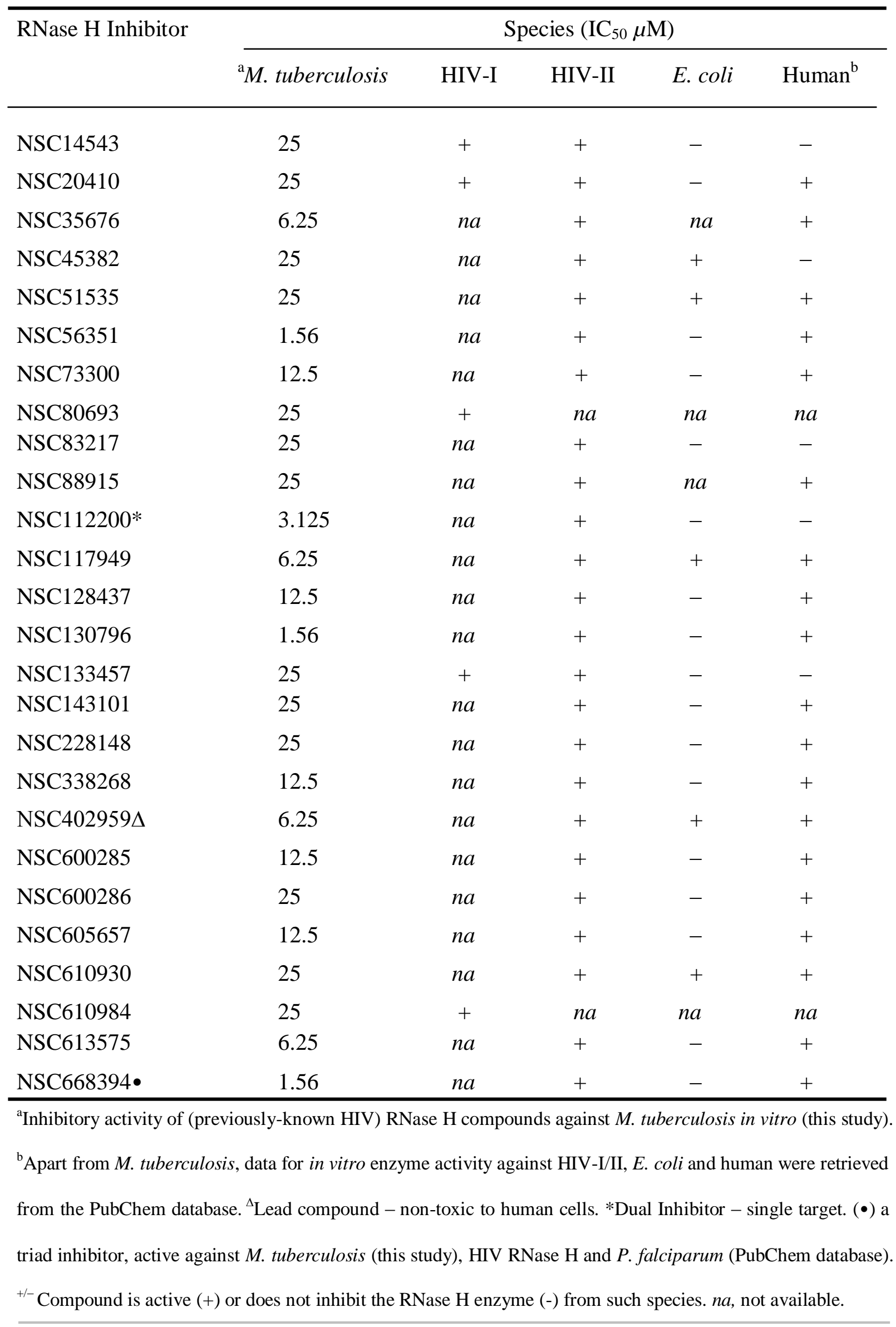


After initial evaluation of anti-RNase $\mathrm{H}$ compounds for their growth inhibitory activity against $M$. tuberculosis H37Ra, compounds were prioritized according to pre-defined criteria (section 5.3.4). Compounds that met the criteria were further characterized, and their activity profiles across other species (clinical strains) are tabulated in tables 5.2 and 5.3. This was also done to confirm whether or not they inhibited E. coli in dose-response assays. To examine this, inhibitors were tested in vitro against three clinical strains (Table 2.1). The compounds were prioritized based on their inhibitory activity against $M$. tuberculosis in vitro and antiHIV RNase H activity. Table 5.2 presents further data for cytotoxicity of individual compounds and their activity profile in nutrient-rich and carbon-starved conditions.

Table 5.2: Anti-mycobacterial Efficacy and toxicodynamics for RNase HI inhibitors of M. tuberculosis

\begin{tabular}{lcccc}
\hline RNase H Inhibitor & \multicolumn{3}{c}{$50 \%$ Inhibitory Concentration $(\mu \mathrm{M})$} & \\
\hline & MW (g/mol) & M. tuberculosis & C-limited Mtb & ${ }^{b}$ HL-60 \\
\hline NSC20410 & 634.02 & 25 & - & - \\
NSC35676 & 220 & 6.25 & - & - \\
NSC56351 & 546.48 & 1.56 & - & - \\
NSC112200 & 590 & 3.125 & - & - \\
NSC130796 & 392.33 & 1.56 & - & $>100$ \\
NSC133457 & 212.19 & 25 & - & 50 \\
NSC143101 & 611 & 25 & - & - \\
NSC338268 & 384 & 12.5 & - & $>100$ \\
NSC402959 & 779.41 & 6.25 & 3.125 & - \\
NSC605657 & 716.5 & 12.5 & - & - \\
NSC613575 & 627.64 & 6.25 & - & $<10$ \\
NSC668394 & 452.1 & 1.56 & - & - \\
\hline
\end{tabular}

Compounds were tested up to a maximum concentration of $100 \mu \mathrm{M}$. Compounds exhibiting inhibitory activity against M. tuberculosis H37Ra up to maximum concentration tested $(100 \mu \mathrm{M})$ are given. For comparison of 
inhibitory profile of the anti-RNase $\mathrm{H}$ compounds against M. tuberculosis $\mathrm{H} 37 \mathrm{Ra}, \mathrm{IC}_{50}$ values for both nutrient rich and nutrient limited conditions are given. . ${ }^{\mathrm{b}} 50 \%$ inhibitory concentrations of RNase HI compounds against HL-60 cells. The symbol (-) indicates, not done. MW, molecular weight.

\subsubsection{Activity of HIV RNase H Inhibitors Against M. tuberculosis RNase HI}

After compounds were tested in the growth inhibition assays against M. tuberculosis H37Ra, all anti-HIV RNase $\mathrm{H}$ compounds were ranked on the basis of their anti-mycobacterial activity. Selective HIV RNase $\mathrm{H}$ inhibitors were then tested in a biochemical assay (at the University of Auckland) to validate their anti-RNase HI activity in M. tuberculosis. Inhibitors were tested at two concentrations of $100 \mu \mathrm{M}$ (being highest) and $5 \mu \mathrm{M}$ (being lowest) to initially evaluate their activity in vitro. Compounds NSC112200 and NSC668394 inhibited the activity of RNase $\mathrm{HI}(\mathrm{Rv} 2228 \mathrm{c})$ in vitro at concentrations $5 \mu \mathrm{M}$ and $100 \mu \mathrm{M}$, respectively (Figure 7.14). Data obtained from biochemical assay for these compounds (Figure 7.14) are consistent with the data received from growth inhibition assays (Table 5.2) and in silico analysis on these inhibitors (Figure 5.10), except that compound NSC668394 was not effective at lowest concentration tested $(5 \mu \mathrm{M})$ compared to NSC112200 suggesting that NSC668394 is not specific to mycobacterial RNase HI and may have a different target in $M$. tuberculosis. However, they displayed good anti-mycobacterial activity against $M$. tuberculosis H37Ra in dose response assays (Table 5.1), and NSC112200 (does not interfere with human enzyme, PubChem database) appears to be anti-M. tuberculosis RNase HI specific (this study). All other inhibitors that displayed good anti-mycobacterial activity in dose response assays are listed in table 5.2.

Apart from NSC112200, NSC133457 was one of potential inhibitors identified that met the objectives of this study i.e. HIV RNase $\mathrm{H}$ inhibitors that exhibit anti-mycobacterial activity 
and do not inhibit human RNase H enzyme (section 5.3.4 and table 5.2). The dose-response curve for NSC133457 is given below (Figure 5.3).

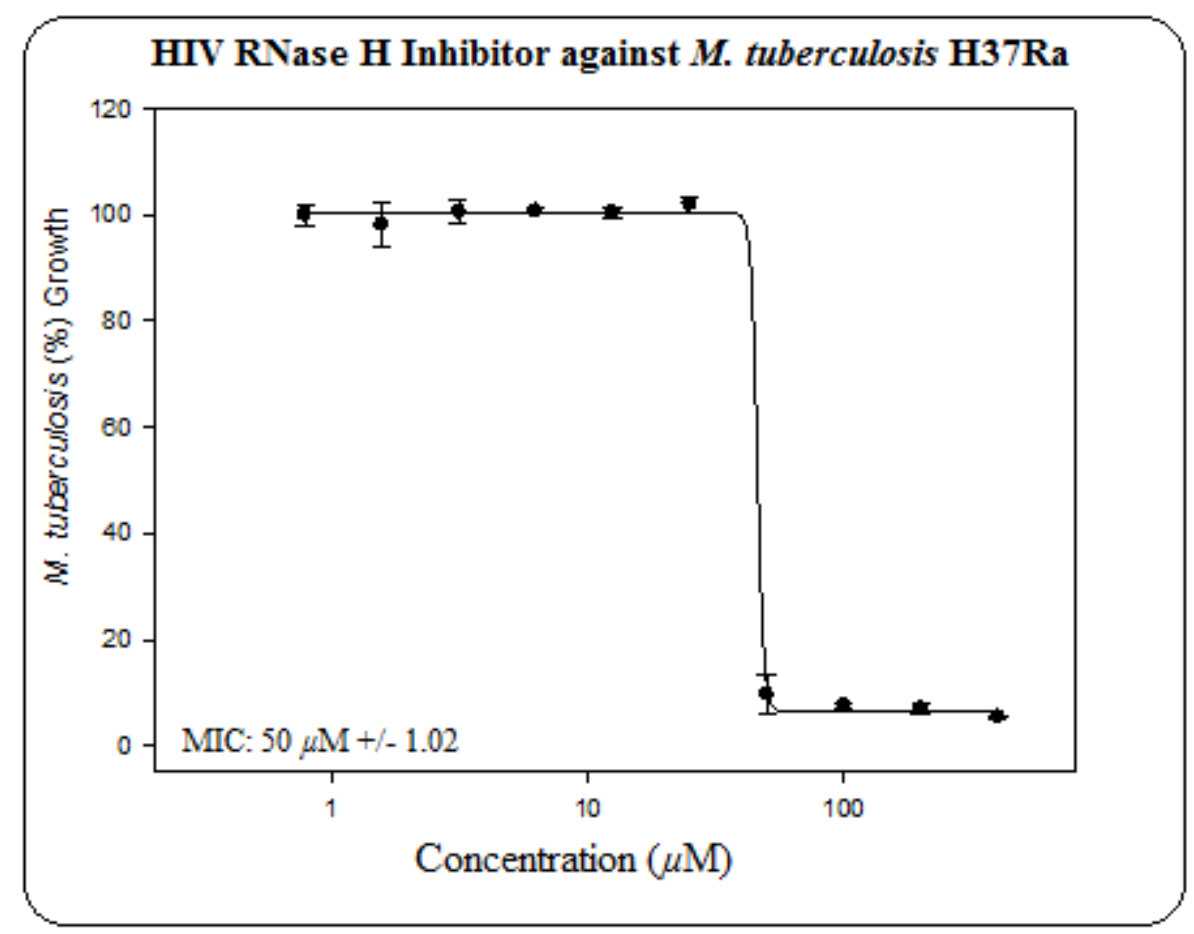

Figure 5.3: Dose-response curve for anti-HIV RNase H compound NSC133457 against M. tuberculosis (see Figure 5.5 for chemical structure of NSC133457).

\subsubsection{Evaluation of the Cytotoxic Properties of the Inhibitors Identified}

Prioritized anti-HIV RNase $\mathrm{H}$ compounds that exhibited inhibitory activity against $M$. tuberculosis in the growth inhibition assay were also counter screened for activity in a human cell line HL-60 in order to specifically identify potential leads that would be non-toxic to human cells. Table 5.2 presents $50 \%$ inhibitory concentrations $\left(\mathrm{IC}_{50}\right)$ of inhibitors compounds tested for cytotoxicity. Compounds NSC133457 and NSC402959 were of paramount importance as they presented favourable toxicities against HL-60 cells (Figure 5.4 and Figure 4.13, respectively) and were also active against $M$. tuberculosis with an $\mathrm{IC}_{50}$ of $25 \mu \mathrm{M}$ and $6.25 \mu \mathrm{M}$, respectively (Table 5.2). 


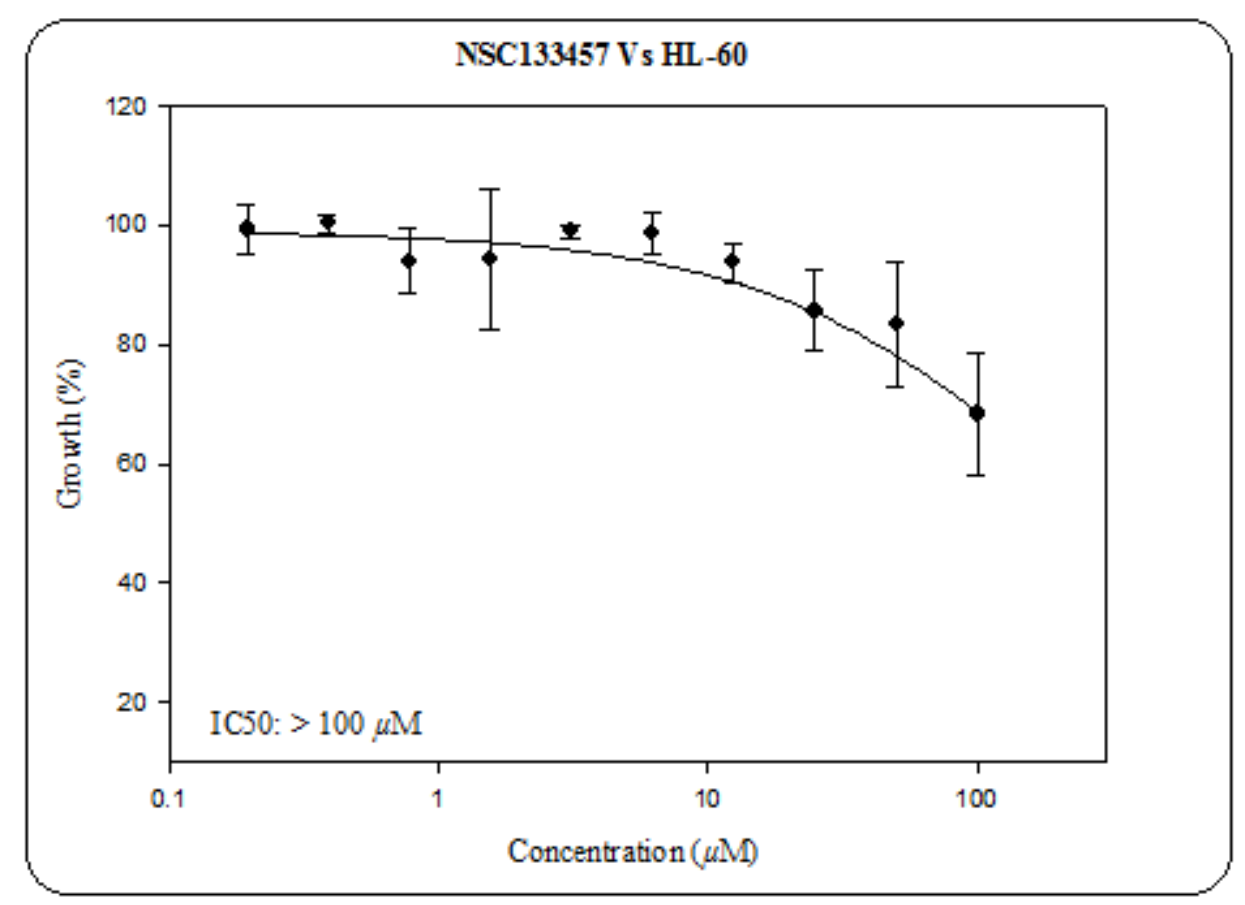

Figure 5.4: Activity of NSC133457 against the human cell line, HL-60.

NSC133457 (Figure 5.5) showed a reasonable anti-mycobacterial activity against $M$. tuberculosis in dose-response assays $\left(\mathrm{IC}_{50} 25 \mu \mathrm{M}\right)$. It is not reported as an RNase $\mathrm{H}$ inhibitor of $E$. coli and also does not inhibit human enzyme (Table 5.1). It was therefore deemed appropriate to further test it against E. coli (Table 5.3). Given that NSC133457 is a known RNase H inhibitor of HIV (PubChem database); we hypothesized that its anti-mycobacterial activity may also be due to its inhibition of the same enzyme in M. tuberculosis (Figure 5.11). Lipinski's rule [251] suggested that an ideal drug will have to have low molecular weight and fewer rings with a smaller number of rotatable bonds (section 5.7), from this prospective, NSC133457 fitted these criteria. While most anti-Tb drugs do not follow Lipinski's rules, these criteria may only serve as guideline in finding compounds with drug-like properties and selecting leads in the initial stages of drug discovery. 


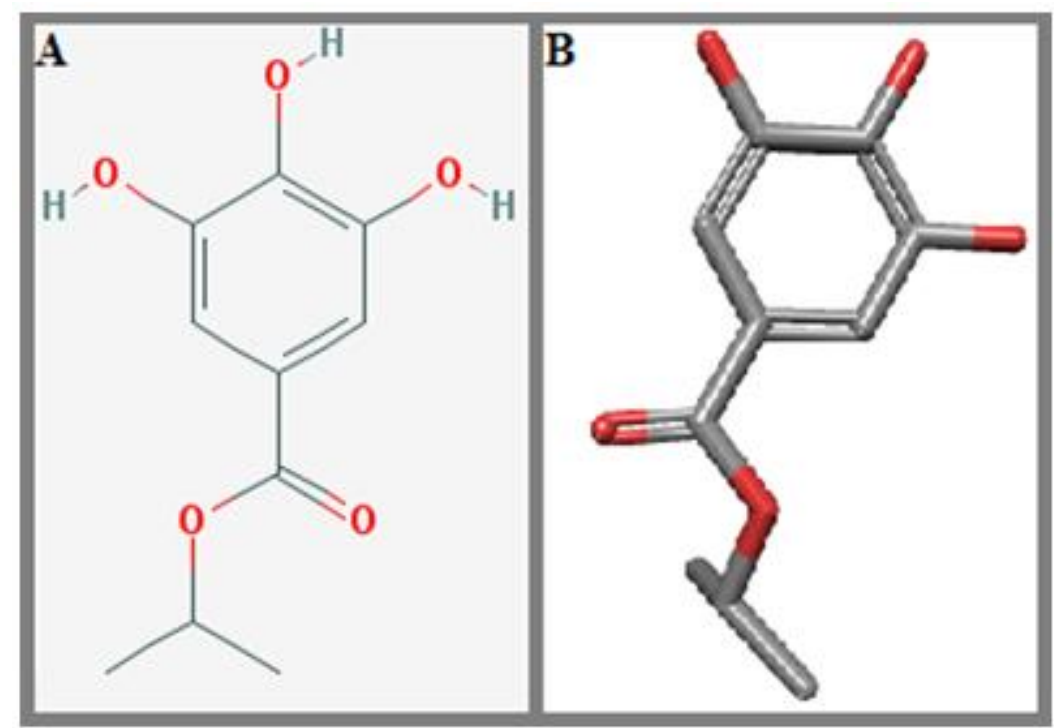

Figure 5.5: Chemical structure of NSC133457. MW $212.19 \mathrm{~g} / \mathrm{mol}$ and $\mathrm{MF} \mathrm{C}_{10} \mathrm{H}_{12} \mathrm{O}_{5}$ (A) 2D structure and (B) $3 \mathrm{D}$ structure.

\subsubsection{Inhibitory Profile of RNase $H$ Compounds against Clinical Strains}

Anti-mycobacterial compounds were also tested for their activity against a number of clinically important pathogens as described in section 2.7.2.3. While compounds displayed good anti-mycobacterial activities, none of the compounds were active against $E$. coli in the growth inhibition assays except NSC402959 (Table 5.3), which is also an inhibitor of E. coli RNase H (PubChem database). Additionally, these compounds were tested against S. aureus and $P$. aeruginosa. None of the compounds tested in dose-response assays were active against $P$. aeruginosa probably due its highly efficient efflux pumps. Therefore, compounds with significant anti-mycobacterial activity were selected for in silico analysis to determine if they specifically bound at the active site of RNase $\mathrm{H}$ enzyme in M. tuberculosis $\mathrm{H} 37 \mathrm{Rv}$ (Figure 5.10).

NSC402959 (Chapter 3) served as an entry point into this new class of anti-Tb agents, and RNase HI (Rv2228c) could well be a viable drug target for such inhibitors, e.g. NSC112200 
displayed good activity against purified Rv2228c and can serve as a potential member of a larger class of (RNase HI) M. tuberculosis inhibitors (Figure 5.10 and Figure 7.14).

Table 5.3: Activity profile of RNase HI inhibitors against clinical strains

\begin{tabular}{lcccc}
\hline RNase H Inhibitor & \multicolumn{4}{c}{ Species (MIC $\mu \mathrm{M})$} \\
\cline { 2 - 4 } & M. tuberculosis & ${ }^{1}$ E. coli & S. aureus & P. aeruginosa \\
\hline NSC20410 & 50 & $>100$ & $>100$ & $>100$ \\
NSC35676 & 12.5 & $>100$ & $>100$ & $>100$ \\
NSC56351 & 3.125 & $>100$ & 12.5 & $>100$ \\
NSC112200 & 6.25 & $>100$ & 12.5 & $>100$ \\
NSC130796 & 3.125 & $>100$ & $>100$ & $>100$ \\
NSC133457 & 50 & $>100$ & $>100$ & $>100$ \\
NSC143101 & 50 & $>100$ & $>100$ & $>100$ \\
NSC338268 & 25 & $>100$ & $>100$ & $>100$ \\
NSC402959 & 12.5 & 50 & $>100$ & $>100$ \\
NSC605657 & 25 & $>100$ & $>100$ & $>100$ \\
NSC613575 & 12.5 & $>100$ & $>100$ & $>100$ \\
NSC668394 & 3.125 & $>100$ & 6.25 & $>100$ \\
\hline NS6 & & &
\end{tabular}

${ }^{1}$ Detail on each clinical strain used in this study, are presented in table 2.1. Data available on PubChem database for individual anti-HIV RNase $\mathrm{H}$ compounds against clinical strains were cross verified by testing these compounds against clinical strains in dose response assays to confirm if they also inhibited growth of these species in vitro. All compounds were tested up to a maximum concentration of $100 \mu \mathrm{M}$. (+) indicates activity of inhibitor in that species and (-) indicates no inhibition detected at $100 \mu \mathrm{M}$. MIC, minimum inhibitory concentration. 


\subsubsection{Cloning of M. tuberculosis RNase H proteins into E. coli BL21 (DE3)}

Cloning experiments were initiated to express RNase HI and RNase HII from M. tuberculosis in the E. coli BL21 expression strain. Figure 5.6 presents the steps of the cloning experiments.

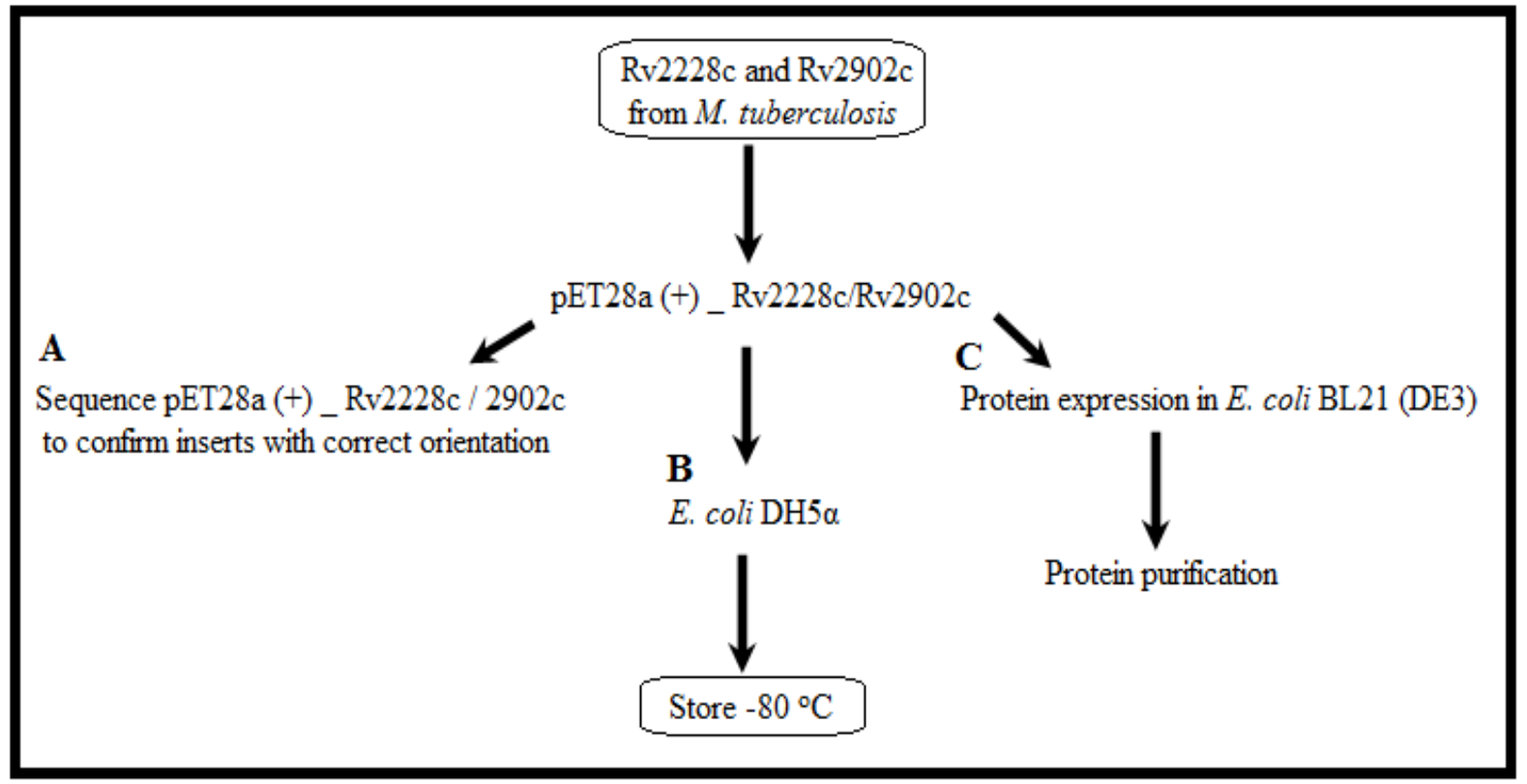

Figure 5.6: Cloning steps for Rv2228c and Rv2902c from M. tuberculosis in the E. coli strains. (A) Confirmation of cloning of Rv2228c in correct orientation through sequencing of pET28a (+)_Rv2228c (B) After confirmation, $-80{ }^{\circ} \mathrm{C}$ freezer stocks of E. coli cells expressing Rv2228c were made and (C) Expression of Rv2228c using the same vector in E. coli BL21 expression strain to over express Rv2228c protein.

\subsubsection{PCR-amplification, Restriction Digestion and Ligation of Rv2228c and Rv2902c into an E. coli Expression Vector, pET28a (+)}

Rv2228c encoding RNase HI in M. tuberculosis was cloned into E. coli BL21 (Figure 5.6) and sequenced (Figure 7.2). Subsequent steps for protein expression were performed as described in the methods section (Chapter 2). Figure 5.7 shows the PCR amplification and restriction digestion of $M$. tuberculosis genes (section 2.7.7.1). The amount of DNA was quantified using a Nano Drop ${ }^{\mathrm{TM}}$. 


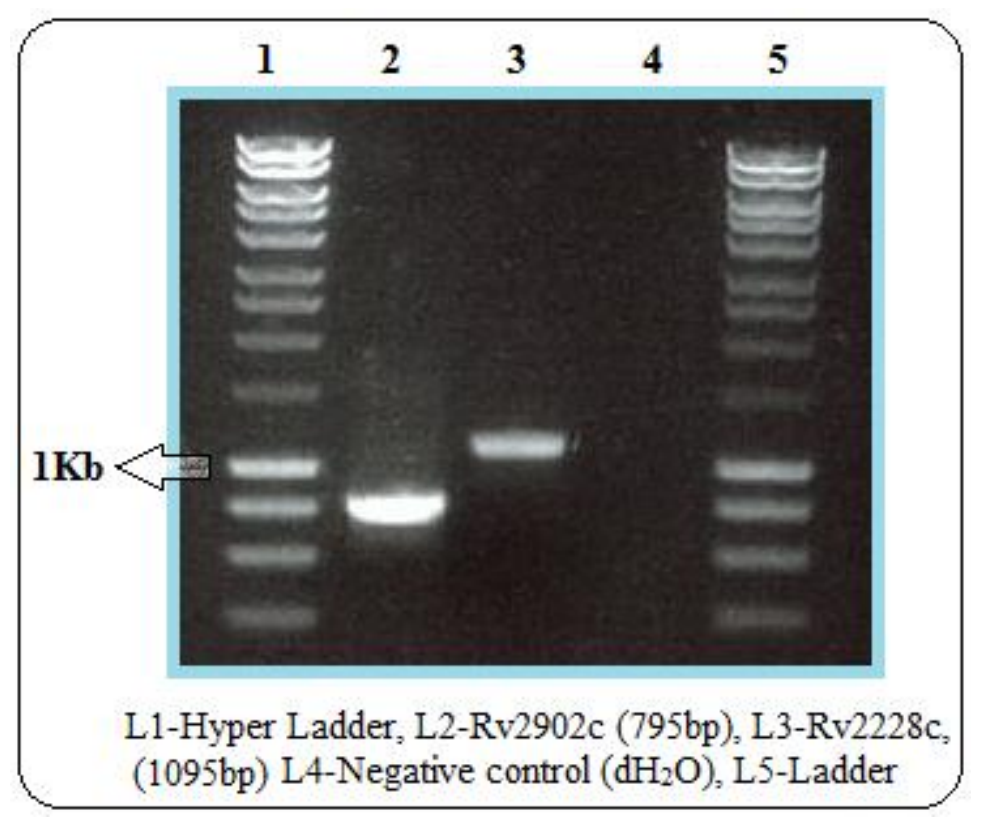

Figure 5.7: Confirmation of PCR Rv2228c and Rv2902c. Sizes of the PCR bands for Rv2902 and Rv2228c were 795 bp (lane 2) and 1095 bp (lane 3), respectively. Detail of primer design and restriction sites are given in table 2.3. All PCR products were analysed as described in section 2.7.7.5. A total of $5 \mu \mathrm{L}$ of sample was loaded on to $1 \%$ molecular biology grade agarose in $1 \mathrm{x}$ TAE buffer stained with ethidium bromide.

Figure 5.8 shows a restricted digest of the PCR product of Rv2228c. Restriction digestions of PCR products and the vector were performed as described in section 2.7.6.7.

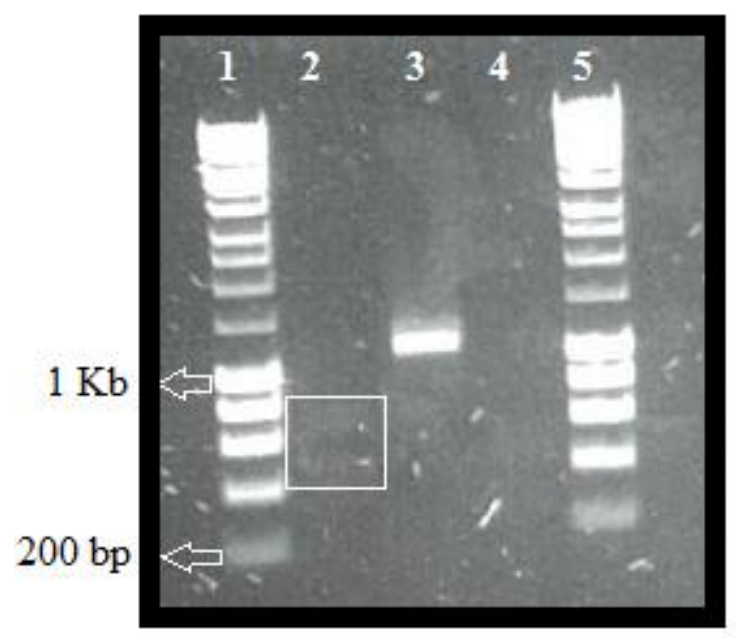

Figure 5.8: Size fractionated analysis of a restriction digest, Rv2228c. The BamHI restriction site was present at position 423 bp in Mtb's ORF Rv2228c. It yielded two bands (lane 2) of sizes 670 bp and 423 bp, 
respectively. Lane 3 shows the band for Rv2228v (PCR-amplified), used as a positive control for the restriction experiment. Lane 4 was a negative control $\left(\mathrm{ddH}_{2} \mathrm{O}\right)$ whereas lane 1 and lane 5 had standard MW ladders.

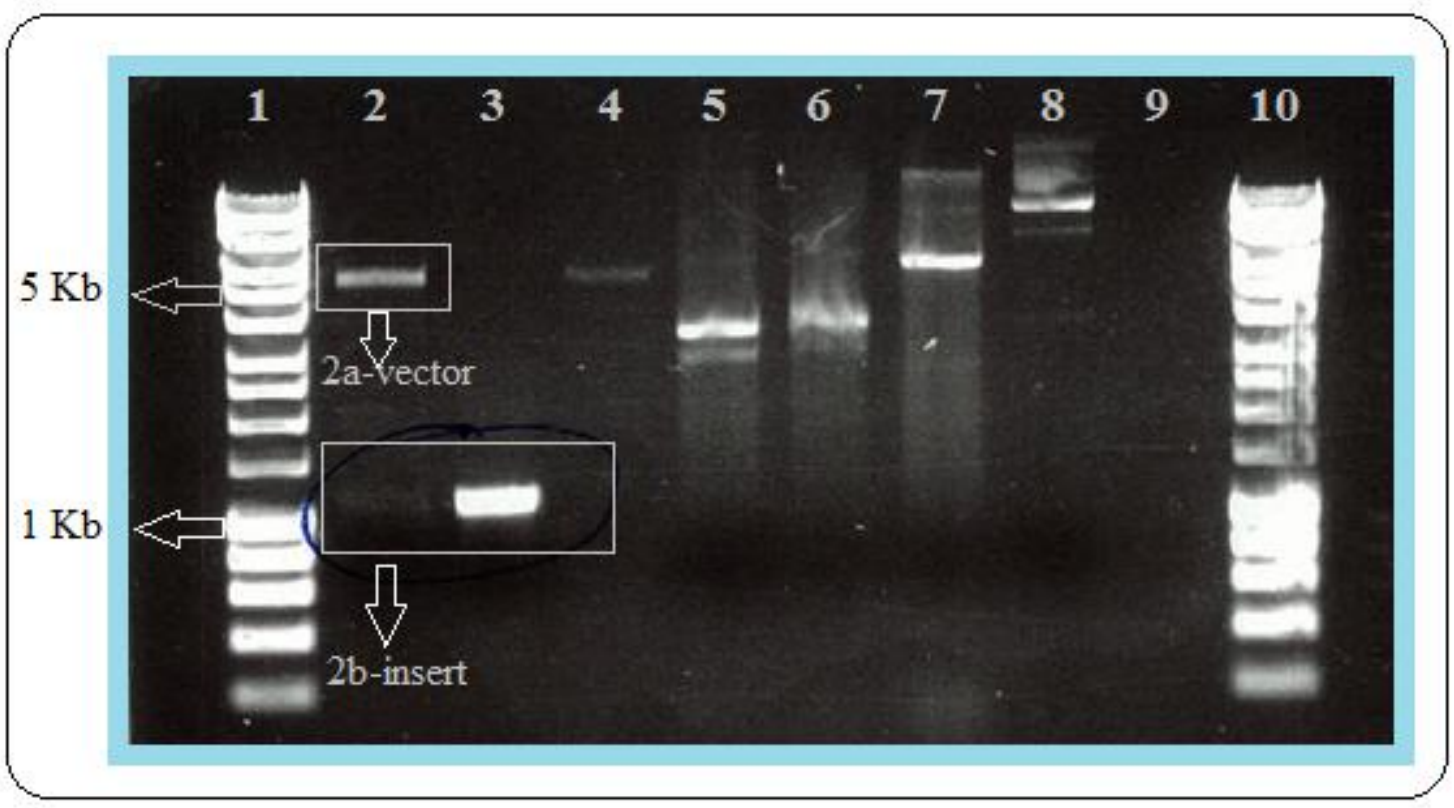

Figure 5.9: Confirmation of ligation of Rv2228c into pET28a. Lanes (1) Hyper ladder showing $1 \mathrm{~kb}$ and $5 \mathrm{~kb}$ bands for insert and vector, respectively (2) A restricted vector pET28a (2a) Digesting the correct size band at the 1095 bp-position (2b) Insert released by the vector pET28a_Rv2228c (+), (3) A control band for insert, PCR-amplifed Rv2228c, product size 1095 bp, (4) Linearized plasmid pET28a (+) digested with EcoRI and XhoI, (5) pET28a (+) uncut - $30 \mathrm{ng} / \mu \mathrm{L}$, fresh stock, (6) pET28a (+) uncut - $28 \mathrm{ng} / \mu \mathrm{L}$, previous stock, (7) pET28a (+) uncut - $20 \mathrm{ng} / \mu \mathrm{L}$, old stock, (8) Control plasmid pCG79 $10 \mathrm{ng} / \mu \mathrm{L}$, (9) No DNA negative control, $\mathrm{dH}_{2} \mathrm{O}$ and (10) Hyper ladder. Approximately $5 \mu \mathrm{L}$ of sample was loaded on to $1 \%$ molecular biology grade agarose in 1x TAE buffer stained with ethidium bromide (sequencing results for Rv2228c are presented in figure 7.2.

\subsection{Molecular Docking Simulations}

In silico analysis of compounds provides insight into which residues of the target they will bind to. To support the idea of developing a single-target dual inhibitor of Tb-HIV, in silico analysis on the novel inhibitors identified in the present study was performed. Chemical 
compounds were docked on the RNase HI protein structure of $M$. tuberculosis $\mathrm{H} 37 \mathrm{Rv}$ using the modelling programmes as described (section 2.8). Table 5.4 presents known HIV RNase $\mathrm{H}$ inhibitors that also interacted with mycobacterial RNase HI. The more negative the total score, the more significant the interaction is.

Table 5.4: Binding scores for interaction of compounds with M. tuberculosis RNase HI

\begin{tabular}{lcccccc} 
RNase H Inhibitor* & \multicolumn{7}{c}{ Binding Scores } \\
\cline { 2 - 7 } & Steric & Desolvation & Acceptor & Donor & Metal & Total Score \\
\hline NSC35676 & -9.266 & 12.981 & -2.493 & -5.562 & -57.117 & -61.456 \\
NSC112200 & -14.218 & 4.959 & -9.803 & 0.00 & -24.503 & -43.563 \\
NSC668394 & -27.329 & 9.827 & -6.391 & -0.389 & -23.481 & -47.763 \\
\hline
\end{tabular}

Compounds that gave the highest binding scores for the proposed binding sites are given. A negative score implies a spontaneous free energy of binding. NSC112200 is a novel anti-Tb compound identified in this study that is also a known HIV RNase $\mathrm{H}$ inhibitor (PubChem database). In silico analysis gave a positive interaction of NSC112200 with the RNase HI domain (Rv2228c) in M. tuberculosis (Figure 5.10). Interaction of inhibitors was studied only with the crystal structure of the RNase $\mathrm{H}$ domain. A more in-depth analysis may reveal missing interaction with the rest of the protein to determine the model's ability to predict stronger interactions. Modelling was carried out using docking software as described in section 2.8. *HIV RNase H inhibitors.

The divalent ions $\mathrm{Mg}^{2+}$ or $\mathrm{Mn}^{2+}$ are required for RNase $\mathrm{H}$ activity and are thus a significant part of the binding simulations in the docking studies; therefore, the metal ions were added computationally to the proposed binding sites on the crystal structure of an RNase HI. Compounds mentioned in table 5.3 were docked on mycobacterial RNase HI but only a few inhibitors got through the selected filters and docked with reasonable binding scores. NSC668394 was found to bind more strongly than NSC112200, and had a comparatively better cumulative score (Table 5.4). However, NSC112200 had a lower desolvation score 
than NSC668394 requiring less energy for its docking on the binding site. Results from the in silico analysis showed that an interaction of these HIV RNase $\mathrm{H}$ inhibitors with the mycobacterial RNase HI was plausible (Figure 5.10). However, the docking score for inhibitors (Table 5.4) was lower than the decoy ligands selected from the Spectrum Collection which were used to examine comparative binding scores with $M$. tuberculosis RNase HI (data not shown), suggesting that either the binding site model is limited or these ligands work through other means.

This was further confirmed by testing selected inhibitors in an enzyme inhibition assay to elucidate their specific inhibitory activity against RNase HI in M. tuberculosis. These findings may eventually help in the development of a new class of $M$. tuberculosis inhibitors. Since these inhibitors are effective against both HIV and M. tuberculosis, they can also be tested for their antagonistic or synergistic activities with other clinical anti-retroviral drugs. 

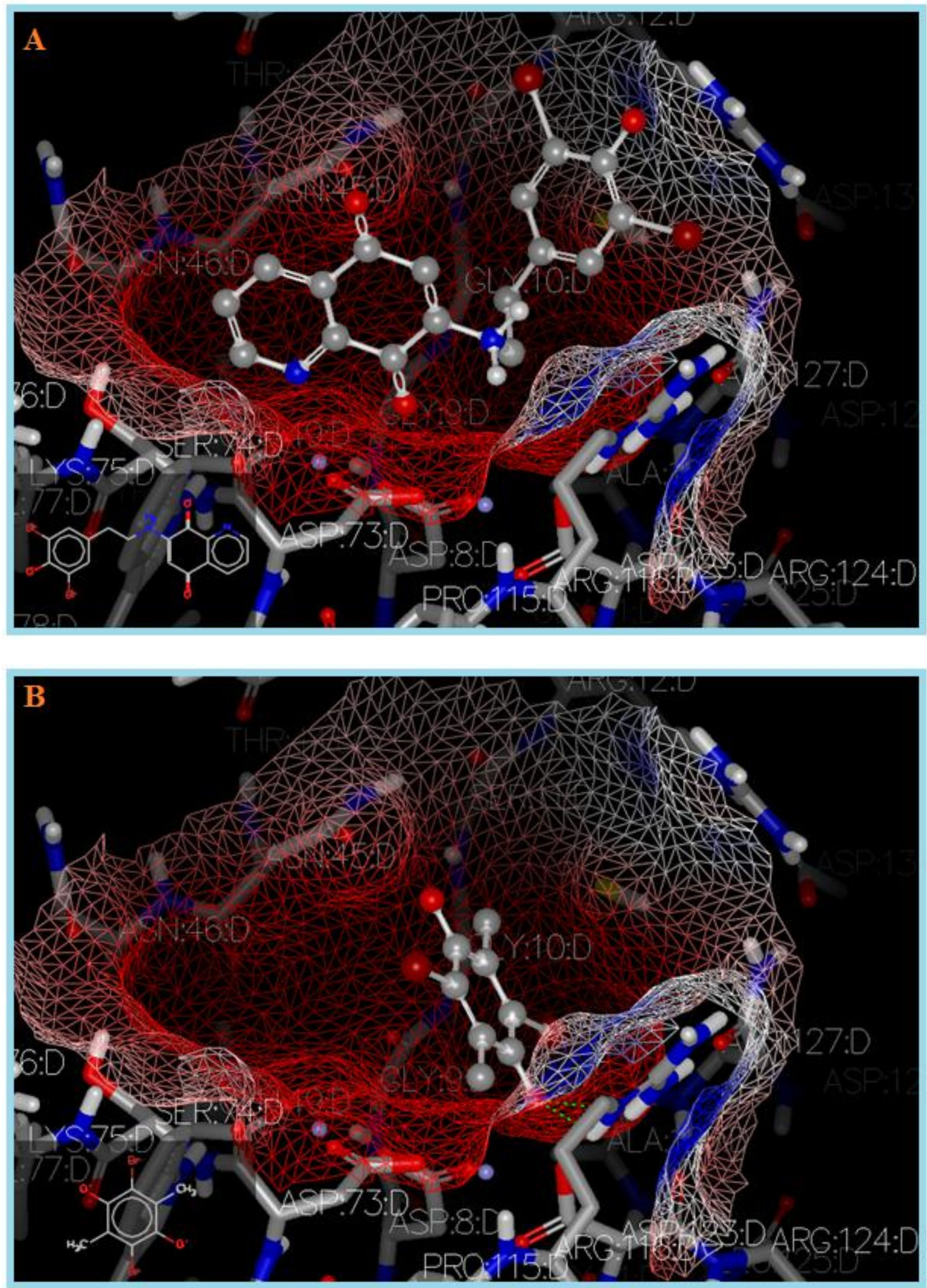

Figure 5.10: Molecular docking of HIV RNase H inhibitors on M. tuberculosis RNase HI (A) NSC668394 and (B) NSC112200. Unlike NSC668394 (MIC of $3.125 \mu \mathrm{M}$ ), NSC112200 does not interfere with the human 
enzyme (Pubchem) but was found active against M. tuberculosis H37Ra with an MIC of $6.25 \mu \mathrm{M}$ (this study). These compounds had better binding scores (Table 5.4) compared to other inhibitors that were docked on to $M$. tuberculosis RNase $\mathrm{HI}$ in the presence of divalent ions $\left(\mathrm{Mg}^{2+}\right)$, suggesting a positive interaction between HIV RNase $\mathrm{H}$ inhibitors and a mycobacterial RNase $\mathrm{HI}$ protein (colours: grey = carbon, red = oxygen, blue nitrogen and white = hydrogen). M. tuberculosis RNase HI = red ribbon. (A) NSC668394 = grey aromatic rings and (B) NSC112200 = a grey ring with two oxygen atoms (red). Regions that interact strongly with a point positive charge are coloured red, those that interact strongly with a point negative charge are coloured blue; whereas, more pale colours denote a weaker electrostatic potential. Figure courtesy of Associate Prof Paul Teesdale-Spittle (also see figure 7.14 for specific activity of NSC112200 against mycobacterial RNase HI in vitro).

\subsection{Discussion}

The HIV epidemic along with high Tb burden is a huge concern for disease control agencies [473]. Drugs with novel mechanisms of action are required as they are expected to be more effective since little natural resistance will have arisen against their targets. One of the main aims of this thesis was to identify inhibitors that may have a novel target; hence, the search for RNase $\mathrm{H}$ inhibitors. This study identified a number of interesting M. tuberculosis inhibitors from previously identified anti-RNase H compounds such as NSC402959 (Chapter 4). It may have a similar target (RNase $\mathrm{H}$ ) in M. tuberculosis in vitro as of HIV and thus it may lead to the development of a new class of inhibitors that have not yet been explored.

Previously-known inhibitors of the HIV RNase $\mathrm{H}$ were found to be active against $M$. tuberculosis H37Ra in this study (Table 5.1), suggesting that they may have the same target in both species. Based on the percentage similarity between HIV and P. falciparum RNase HI proteins, these compounds were expected to have an inhibitory activity against malarial species as well [474]. One such compound NSC668394 (Table 5.3) is a known M. tuberculosis inhibitor (MIC $3.125 \mu \mathrm{M}$ in the present study) and is also active against $P$. 
falciparum (PubChem database), (section 6.3.2). NSC668394 and NSC112200 interacted with mycobacterial RNase HI in computer docking studies (Figure 5.10) and this was further confirmed by an in vitro enzyme inhibition assay (section 5.3.3), (Figure 7.14).

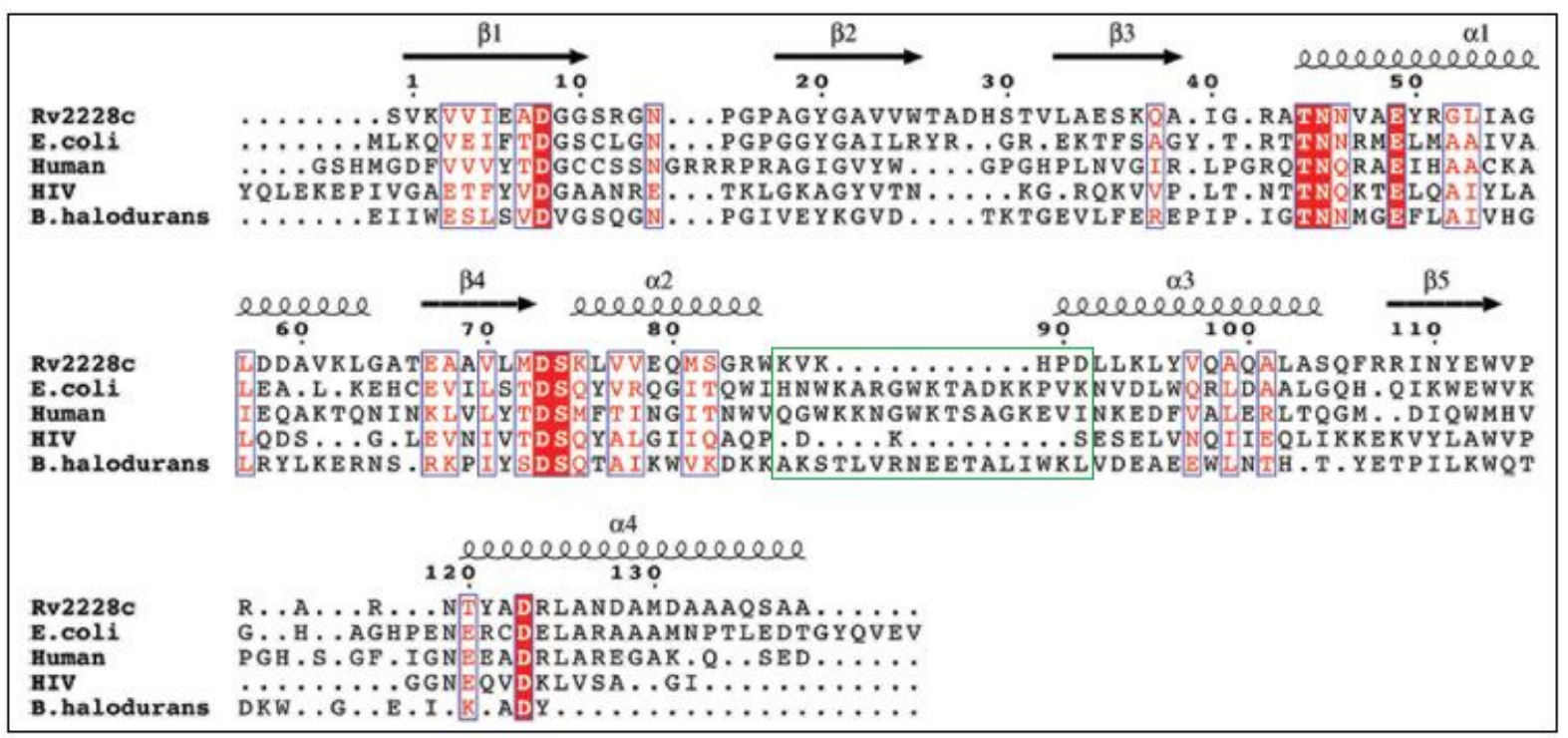

Figure 5.11: Analysis of sequence similarity between Rv2228/N and its structural homologues. Green box reveals the differences in sequence alignments of Rv2228c (involved in recognition), human and HIV RNase H between $\alpha 2$ and $\alpha 3$ helices, which may have implications for determining the specificity of RNase $\mathrm{H}$ inhibitors. Figure was adapted with permission from Watkins et al. [420].

\section{Novel Class of Mycobacterial Inhibitors}

Several anti-HIV RNase H compounds exhibited a consistent inhibitory activity against $M$. tuberculosis $\mathrm{H} 37 \mathrm{Ra}$ with an $\mathrm{IC}_{50}$ of $25 \mu \mathrm{M}$ or less (Table 5.1) e.g. NSC14543, NSC20410 and NSC51535. The structures and molecular weights of these compounds are not very similar (Figure 5.19) yet they all inhibit HIV RNase HI enzyme (PubChem database). NSC133457 (propan-2-yl 3, 4, 5-trihydroxybenzoate) is a colourless compound (Figure 5.5) that has been previously tested in several biological assays but not against M. tuberculosis. It is a known RNase $\mathrm{H}$ inhibitor of HIV type 1 and type 2 and does not inhibit human RNase $\mathrm{H}$ 
(PubChem database). Given its considerable inhibitory activity against M. tuberculosis, it is an attractive lead that could be further characterized and subsequently developed as a new inhibitor for Tb and HIV (section 5.4.3). Furthermore, it presented no toxicity towards human cell lines (Figure 5.4). Since in vitro biochemical assays are more sensitive than growth inhibition assays [475], active compounds in biochemical assays may only show a slight inhibition of bacterial growth [476]. Therefore, validation of anti-M. tuberculosis-specific activity of these compounds was confirmed by a biochemical analysis.

Anti-mycobacterial compounds that are highly active against $M$. tuberculosis in vitro may not retain good in vivo activity [343]. The lack of an in vivo activity might be due to involvement of the host immune system, the specific environment of the infected tissue or the amount and type of cells present in that tissue. It is therefore important to evaluate the in vivo efficacy of the validated in vitro inhibitors. This can be tested in suitable in vivo models or in ex vivo models, which mimic the conditions of an in vivo system. For example, Turner et al. have described an ex vivo model that could potentially predict the in vivo effectiveness of inhibitors [477].

NSC402959 identified in this study (Chapter 4), has proven to be a possible entry point to this novel class of M. tuberculosis inhibitors. NSC402959, however, interferes with human RNase $\mathrm{H}$ activity (PubChem database) and may therefore have to go through structural modification before it could be used clinically. NSC133457 however, meets the criteria set up for this project (section 5.3.4) and is thus a more promising candidate for a lead compound. 


\section{Anti-HIV RNase H Compounds}

The criteria used to initially select anti-HIV RNase H compounds in this study were that they exhibit decent anti-mycobacterial activity against $M$. tuberculosis and do not inhibit human RNase H proteins. Many compounds that are known HIV RNase H inhibitors exhibited good inhibitory activity against $M$. tuberculosis and did not inhibit human RNase H enzyme. Examples included NSC14543, NSC45382, NSC83217, NSC112200 and NSC133457. Other compounds e.g. NSC20410, NSC35676, NSC112200, NSC402959 and NSC668394, exhibited good anti-mycobacterial activity in vitro (Table 5.2) but were also active against human RNase H (PubChem database). A few compounds (NSC80693 and NSC610984) may not have been tested against human or $E$. coli RNase $\mathrm{H}$ at all but were active against HIV-I RNase $\mathrm{H}$ and had an $\mathrm{IC}_{50}$ against $M$. tuberculosis of $25 \mu \mathrm{M}$. Such compounds could possibly be chemically modified to alter their activity against human $\mathrm{RNase} H$, while retaining decent inhibitory activity against the mycobacterial and HIV RNase H.

Based on the structural similarity of HIV's RNase H and integrase, Andre'ola et al. have suggested that RNase $\mathrm{H}$ inhibitors may also have anti-HIV integrase activity [463]. In support of this, Pommier et al. have recently patented their approach to treating HIV infections with the compounds NSC18806 and NSC310618 describing them as inhibitors of HIV integrase. From this perspective, if the RNase H inhibitors NSC133457 and NSC402959 (identified in this study) do cause specific inhibition of RNase $\mathrm{H}$ in $M$. tuberculosis, which has homology with HIV RNase [420], it could also be proposed that HIV integrase/RNase $\mathrm{H}$ dual targeting is possible by such anti-RNase $\mathrm{H}$ compounds. Marchand et al. have demonstrated a dual effect of madurahydroxylactone derivatives on the above two enzymes [478]. Therefore, based on the structural similarities of inhibitors, it may be possible to target integrase and RNase H simultaneously. 


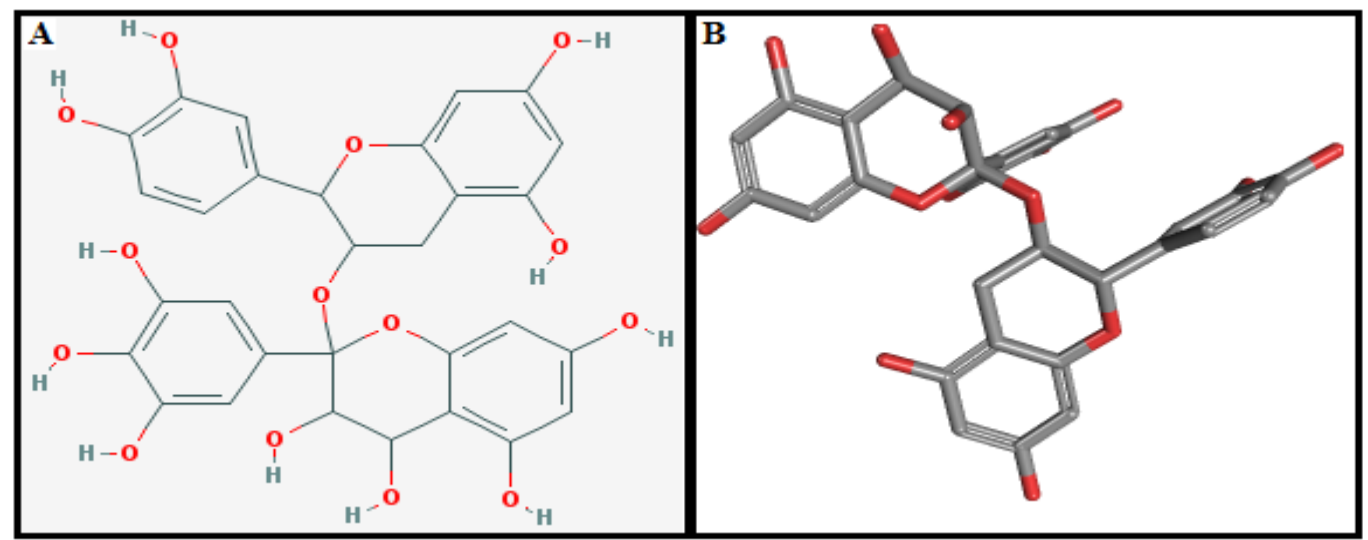

Figure 5.12: Chemical structure of NSC143101, $\mathrm{MF} \mathrm{C}_{30} \mathrm{H}_{26} \mathrm{O}_{14}, \mathrm{MW} 610.52 \mathrm{~g} / \mathrm{mol}$ (A) 2D structure and (B) 3D structure.

NSC133457 (Figure 5.5) and NSC143101 (Figure 5.12) have an MIC of $50 \mu \mathrm{M}$ against $M$. tuberculosis (Table 5.3). At least 5-fold and 2-fold difference was observed between the MICs against $M$. tuberculosis and HL-60 cells, respectively. Anti-M. tuberculosis activity and favourable toxicity make such compounds important to be considered for further development in collaboration with Global Alliance for Tb Drug Development. Furthermore, a programme of chemical derivatisation could be pursued to further strengthen their antimycobacterial activity.

\section{Efficacy of anti-HIV and Novel Inhibitors of M. tuberculosis}

After the initial discovery of active compounds, a range of in vitro research methods have been developed in order to rank novel compounds for their therapeutic potential before they are to be tested against in vivo models. For example, hypoxic conditions in tubercle granuloma have been modelled in vitro using an oxygen depletion assay [122-123]. In addition, lack of several key nutrients used in nutrient starvation assays may mimic in vivo models for nutrient stress [479]. Other in vivo stress conditions include M. tuberculosis growth in an acidic environment and nitric oxide treatment. This unfavourable environment 
for M. tuberculosis replication may help to explain phenotypic drug resistance of these cells and may also explain the altered gene expression of bacteria [480-481]. It is important to completely evaluate the differential anti-mycobacterial effect of new inhibitors between in vitro drug susceptibility assays and an intra-macrophage infection assay in order to examine their potential to invade human macrophages and specifically target latent $M$. tuberculosis. These properties may vary considerably between drugs [482]. The inhibitors identified in the present study were tested under carbon-limited medium conditions for inhibitory activity against $M$. tuberculosis H37Ra, but potential inhibitors must eventually be tested in vivo to completely scale and evaluate their efficacy.

\subsection{Structure-Activity Relationship Study}

Novel anti-Tb agents are required to have a good lipophilicity to be able to penetrate through the lipid-enriched cell wall of $M$. tuberculosis (Figure 7.11) [379-380]. This cell wall is known to have specialized barriers for selective transportation of solutes and drugs compared to other bacteria [483]. New compounds identified through primary screenings with antimycobacterial activity and reduced mammalian cell toxicity could be made more promising drug candidates by performing chemical modifications to increase their lipophilicity and antimycobacterial action. A SAR study is an important step in drug discovery. Any chemical compounds or drugs identified as an inhibitor in mycobacteria are also likely to have some toxicity in human cells. It is therefore important to perform SAR studies in order to design more effective and less toxic inhibitors through derivatisation to generate more effective analogues by making structural modifications that do not compromise their anti-bacterial activity. For example, ethambutol is a frontline ant-Tb drug (Table 1.2) that affects arabinogalactan synthesis in the M. tuberculosis cell wall (Table 1.3) and is considered to be the least effective among four standard drugs prescribed in the initial therapy against $\mathrm{Tb}$. 
SQ109 is structurally similar to ethambutol (Figure 5.13) but has a much higher potency compared to ethambutol. The in vivo efficacy of SQ109 in a mouse model is $1 \mathrm{mg} / \mathrm{kg}$ compared to that of ethambutol, which is $100 \mathrm{mg} / \mathrm{kg}$ [484-485]. Ethambutol-derived analogues have also shown better inhibitory activity than ethambutol [486], suggesting that enhanced inhibition can be obtained by performing structural modification. Chemical modification of compounds may therefore enhance the inhibitory activity and can generate analogues that are more effective than the parent compound.

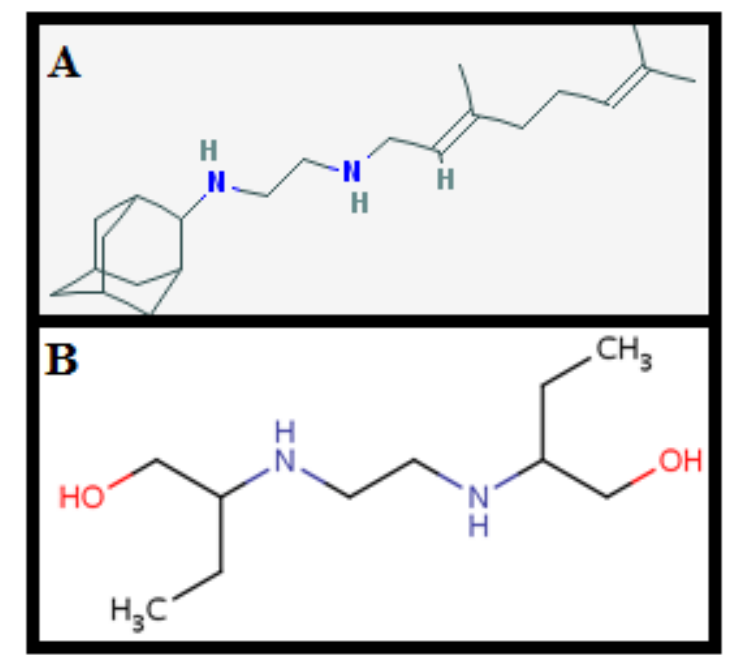

Figure 5.13: Structures of anti-Tb drugs. (A) SQ109 and (B) its structural analogue ethambutol.

Despite better pharmacokinetics, none of the current anti-Tb drugs follow these rules unlike the anti-viral drugs e.g. acyclovir, which matches the rules better. However, given their high anti-mycobacterial activity, inhibitors identified in the present study have the potential to be lead compounds (Table 5.2) as they fulfil two of the main criteria described by Lipinski [251], namely their molecular weights are under $500 \mathrm{~g} / \mathrm{mol}$ and they have $<3$ rotatable rings. 


\subsection{SAR of RNase $H$ Inhibitors}

A brief structural analysis was performed in this study on of HIV-RNase $\mathrm{H}$ inhibitors that were identified as being also active against $M$. tuberculosis H37Ra in this study. The structures were compared on the basis of chemical backbone, with known anti-HIV RNase H compounds to evaluate their likely therapeutic potential. This comparison showed a significant structural similarity between the compounds, suggesting they may be targeting a common enzyme in M. tuberculosis and HIV (Figures 5.15-5.20).

\subsubsection{Comparison of HIV and M. tuberculosis Inhibitors}

Only a few RNase $\mathrm{H}$ inhibitors of HIV have been reported to date, including the diketo acids [487], pyrimidinol carboxylic acids [488], hydroxylated tropolones [489], natural products like illimaquinone [490] and N-acyl hydrazones [491], which effectively inhibit the RNase HI activity of an HIV reverse transcriptase enzyme (HIV-RT). Gong et al. have recently described the interaction of acylhydrazones with HIV-RT [460], comparing their activity with existing inhibitors in order to identify the specific potential of these novel compounds. Acylhydrazones have previously been described to have anti-inflammatory and antithrombotic activity [492]. Moreover, a recent study by Ming et al. suggests that such compounds also possess anti-HIV-Ca activity, and thus like acylthiourea [493], are active against viral capsid proteins [494]. 


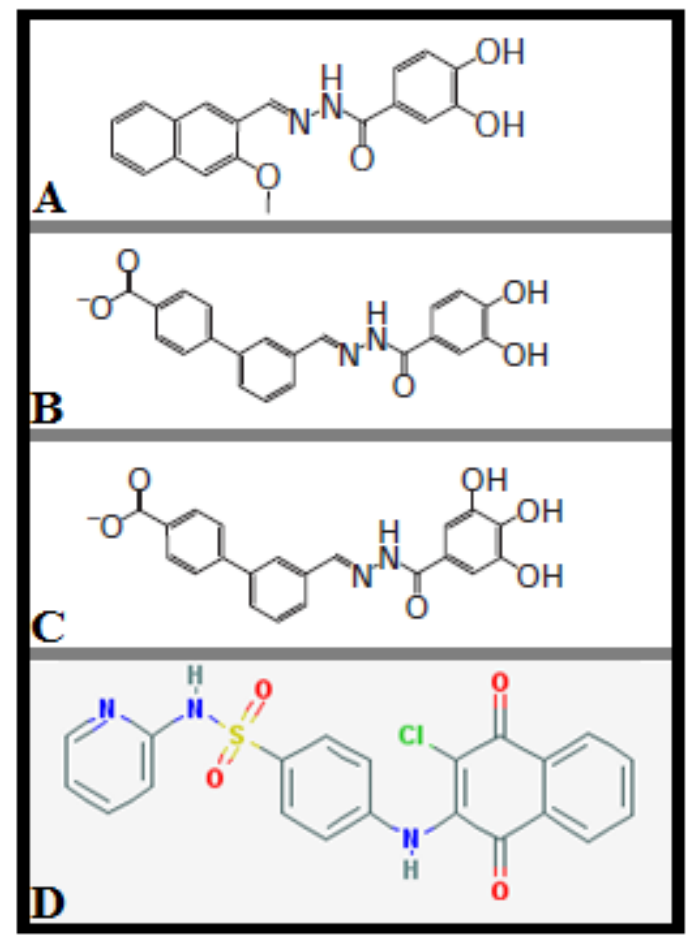

Figure 5.14: Structural comparison of acylhydrazone HIV inhibitors, reported previously, with the $M$. tuberculosis inhibitors identified in this study. (A) Dihydroxy benzoyl naphthyl hydrazone (DHBNH) [495], (B) A dihydroxy analogue of DHBNH (BHMP03) [460], (C) A trihydroxy analogue of DHBNH (BHMP07) [460] and (D) An anti-mycobacterial compound identified in this study, NSC45382, and also previously known as HIV-RNase $\mathrm{H}$ inhibitor (PubChem database). Compound 'C' with a carboxylate moiety in the distal phenyl ring, appeared to have the highest anti-viral activity [460]. This may be due to an extra ' $R$ ' group or the functional group $[\mathrm{OH}]$ in its chemical structure. All compounds otherwise appear to be structurally similar (section 5.8.2). In this study NSC45382, ( $\mathrm{IC}_{50}$ for $M$. tuberculosis of $25 \mu \mathrm{M}$ ), has a molecular weight of 439.87 $\mathrm{g} / \mathrm{mol}$ and has an additional sulphur and chloride group, yet it was found to have structural homology with these acylhydrazone inhibitors.

The compounds shown in figure 5.14 (A, B and C) are derivatives of acylhydrazones [460], and they have been recently reported as anti-HIV RNase H compounds. These compounds bear structural resemblance to the RNase $\mathrm{H}$ inhibitors of HIV identified in this study, such as NSC45382. For example, NSC45382 has a reactive alkyl amine group (Figure 5.14-D), which is not recommended for a prodrug [496] and may need to be chemically removed prior 
to further development of the compound. Another example includes NSC130796, which had an $\mathrm{IC}_{50}$ of $1.56 \mu \mathrm{M}$ against M. tuberculosis $\mathrm{H} 37 \mathrm{Ra}$ (Figure 5.15).

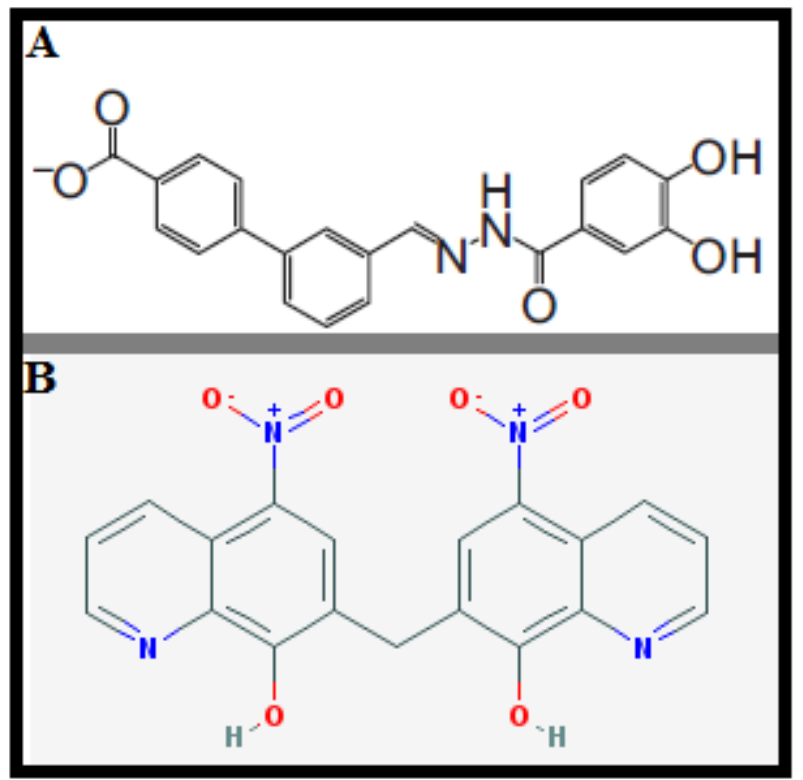

Figure 5.15: Structural comparison of the (A) Acylhydrazone derivative with the (B) Mycobacterial inhibitor identified in this study, NSC130796, MF $\mathrm{C}_{19} \mathrm{H}_{12} \mathrm{~N}_{4} \mathrm{O}_{6}$, MW $392.33 \mathrm{~g} / \mathrm{mol}$. Chemical structure of a dihydroxy analogue of an acylhydrazone derivative was retrieved from Gong et al. [460].

\subsubsection{HIV RNase H Inhibitors that Do Not Inhibit the Human Enzyme}

From the NIH library NSC45382 (4-[(3-chloro-1,4-dioxonaphthalen-2-yl)amino]-N-pyridin2-ylbenzenesulfonamide), NSC83217 (3-amino-4-[(2-amino-4-sulfamoylphenyl)disulfanyl] benzenesulfonamide) and NSC112200 (2,5-dibromo-3,6-dimethylbenzene-1,4-diol) demonstrated reasonable anti-mycobacterial activity in dose-response assays against $M$. tuberculosis H37Ra (Table 5.1). These compounds are of particular importance because of their lack of inhibitory activity against human RNase H proteins. 


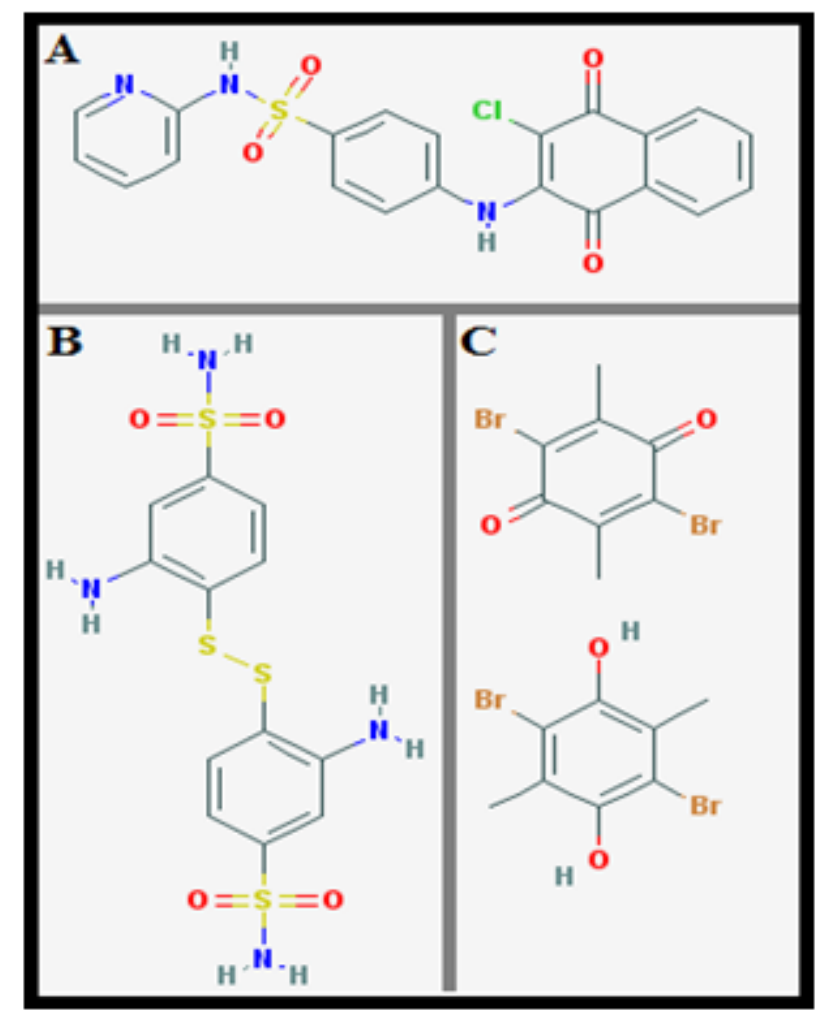

Figure 5.16: Chemical structures of (A) NSC45382, (B) NSC83217 and (C) NSC112200. MWs and MFs are $439.88 \mathrm{~g} / \mathrm{mol}-\mathrm{C}_{21} \mathrm{H}_{14} \mathrm{ClN}_{3} \mathrm{O}_{4} \mathrm{~S}, 406.52 \mathrm{~g} / \mathrm{mol}-\mathrm{C}_{12} \mathrm{H}_{14} \mathrm{~N}_{4} \mathrm{O}_{4} \mathrm{~S}_{4}$ and $589.89 \mathrm{~g} / \mathrm{mol}-\mathrm{C}_{16} \mathrm{H}_{14} \mathrm{Br}_{4} \mathrm{O}_{4}$, respectively.

A more interesting HIV RNase $\mathrm{H}$ inhibitor NSC133457, identified in the present study, exhibited a reasonable anti-mycobacterial activity (Figure 5.3) and did not inhibit human RNase H (PubChem database). It also did not exhibit toxicity towards human cells (Figure 5.4). Furthermore, it appeared to be remarkably similar to the previously reported anti-HIV compounds NSC727447 and $\beta$-thujaplicinol. NSC133457 has a low molecular weight (212.19 $\mathrm{g} / \mathrm{mol}$ ) and has less than two rings in its structures (Figure 5.17-D), and is in agreement with the two important Lipinski rules for demonstrating drug-like properties of a compound (section 2.6.8.1). 


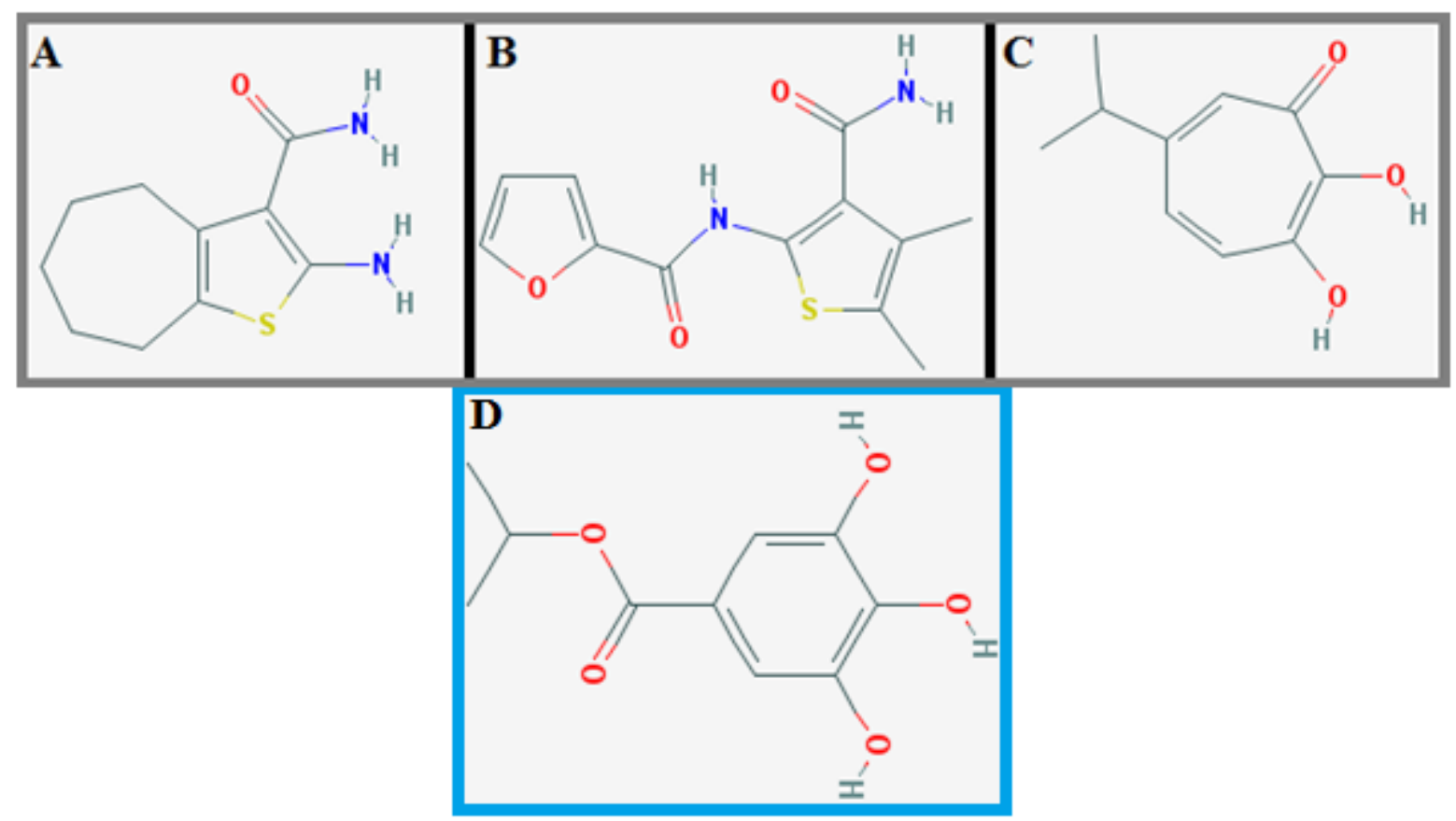

Figure 5.17: SAR of RNase $H$ inhibitors identified in this study with previously reported compounds (A) NSC727447, (B) NSC727448 (C) $\beta$-thujaplicinol and (D) NSC133457. Wandeler et al. have previously identified compounds (A) NSC727447 and (B) NSC727448 from the NIH library as inhibitors of the RNase H enzyme from HIV [470]. (C) $\beta$-thujaplicinol has also been reported as an effective RNase $\mathrm{H}$ inhibitor of HIV [489]. NSC133457 (D) (blue square) was identified as an anti-mycobacterial inhibitor in the present study and shares structural resemblance with the previously reported anti-HIV inhibitors.

Figure 5.17 illustrates the structural similarity among inhibitors. In particular, $\beta$-thujaplicinol and NSC133457 share considerable homology in their chemical structures (Figure 5.17-C and $\mathrm{D}$, respectively). With one benzene ring along with common $\mathrm{OH}$ groups and their low molecular weights, a closer relationship is suggested in terms of their structure and their common inhibition of the RNase H in HIV. Given these structural similarities and the lack of published reports on anti-M. tuberculosis activity with the current and previously-known drugs, NSC133457 offers excellent potential to be developed further (section 5.4.3). 


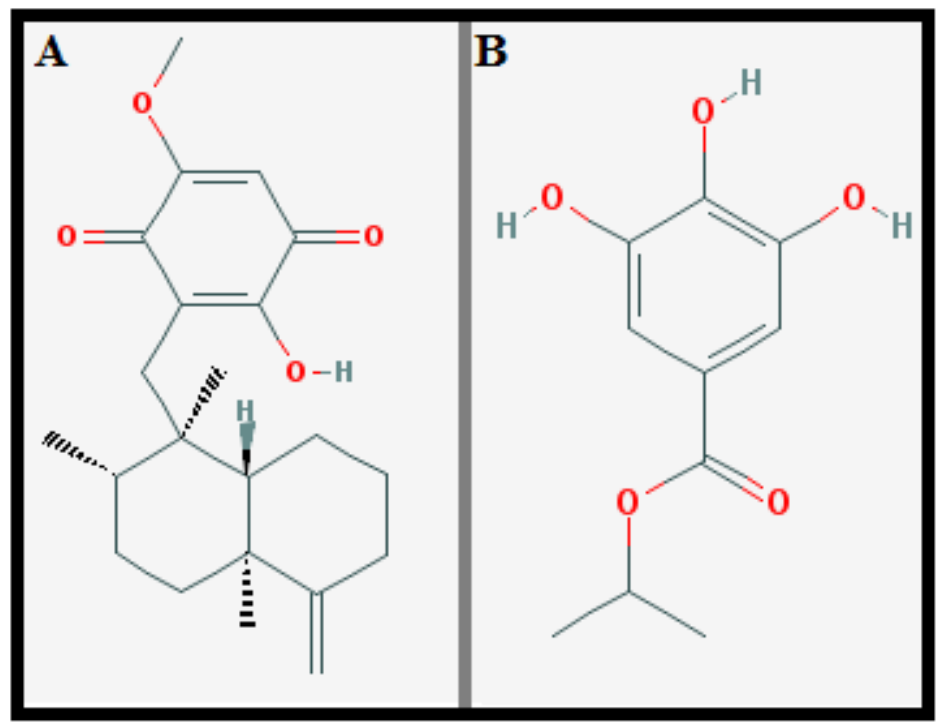

Figure 5.18: Structural similarity between an HIV RNase H inhibitor (A) illimaquinone and a mycobacterial inhibitor, (B) NSC133457. $\mathrm{MW}$ for ' $\mathrm{A}$ ' and 'B' are $358.48 \mathrm{~g} / \mathrm{mol}$ and $212.19 \mathrm{~g} / \mathrm{mol}$, respectively (section 5.8.1).

NSC14543 (3,5-bis[(dimethylamino)methyl]-1,3,5-thiadiazinane-2-thione), NSC20410 ((1,3dimethyl-2,6-dioxopurin-7-yl)-[2-methoxy-3-(1,1,3-trioxo-1,2-benzothiazol-2-

yl)propyl]mercury) and NSC51535 (3,12-dinitroanthra[9,1,2-cde]benzo[rst]pentaphene-5,10dione) have different chemical structures and formula weights (Figure 5.19), yet these inhibitors exhibited a consistent anti-mycobacterial activity in dose-response assays against M. tuberculosis H37Ra (section 5.6). However, apart from inhibiting HIV-RNase H, all compounds presented in figure 5.19 (except NSC14543), also inhibit the activity of human enzyme (Table 5.1). An additional structural modification to these compounds may yield more effective derivatives with increased efficacy, and therefore such inhibitors may have to be chemically modified. 


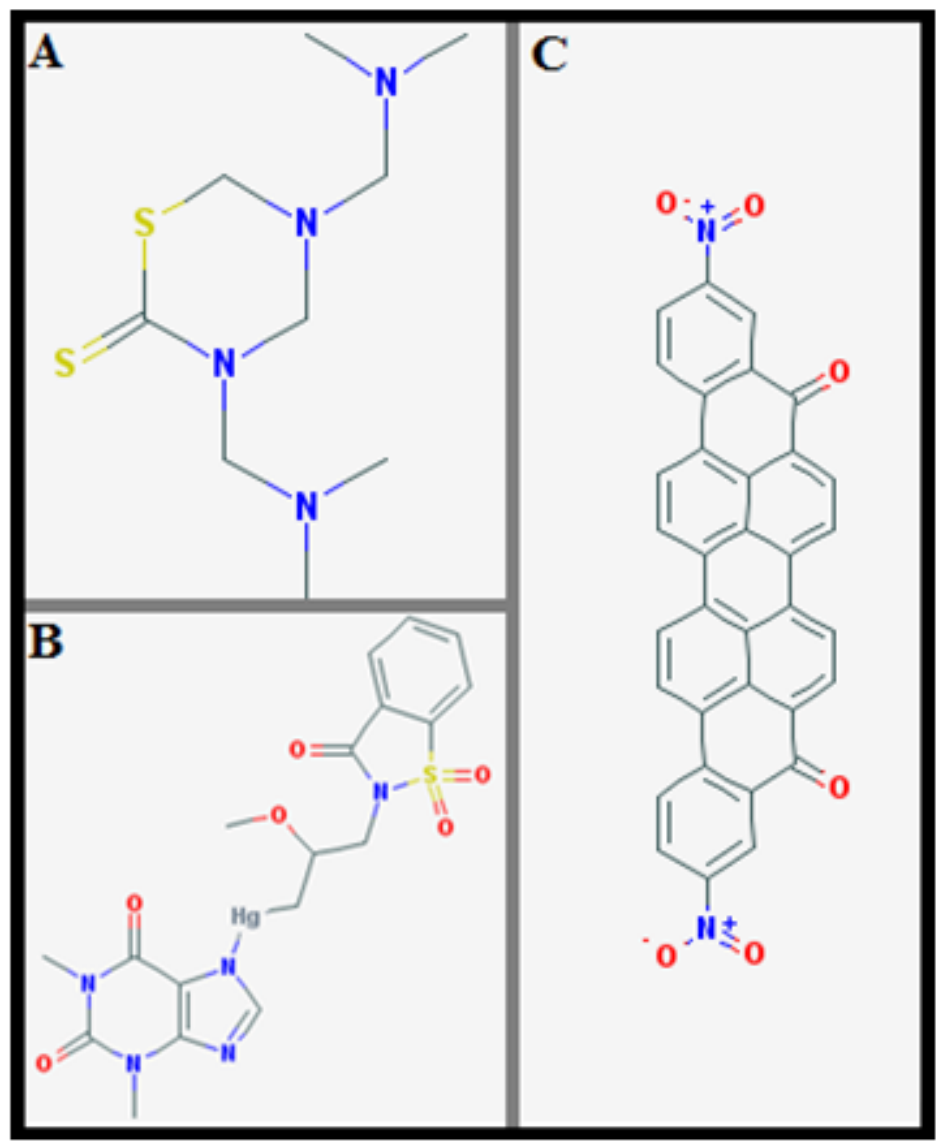

Figure 5.19: Chemical structures of (A) NSC14543, (B) NSC20410 and (C) NSC51535. MW and MF of the compounds are (A) $248.41 \mathrm{~g} / \mathrm{mol}-\mathrm{C}_{9} \mathrm{H}_{20} \mathrm{~N}_{4} \mathrm{~S}_{2}$, (B) $634.02 \mathrm{~g} / \mathrm{mol}-\mathrm{C}_{18} \mathrm{H}_{19} \mathrm{HgN}_{5} \mathrm{O}_{6} \mathrm{~S}$ and (C) $546.48 \mathrm{~g} / \mathrm{mol} \mathrm{-}$ $\mathrm{C}_{34} \mathrm{H}_{14} \mathrm{~N}_{2} \mathrm{O}_{6}$, respectively [497].

\subsubsection{Effect of Hydroxyl Groups on the Activity of Mycobacterial Inhibitors}

All compounds with reactive hydroxyl groups $[\mathrm{OH}]$ were expected to exhibit some inhibitory activity against $M$. tuberculosis H37Ra. Streptomycin (Figure 7.10-A) is one of the most active second-line anti-Tb drugs and it has seven hydroxyl groups in its structure. An $\mathrm{OH}$ group at position 12 may be of critical importance in terms of it being anti-mycobacterial, as shown previously by Xu et al. [498]. Xu et al. identified a new compound, (+)-calanolide A, which is a natural product with anti-HIV properties. This compound was further tested against $\mathrm{Tb}$ strains and was also shown to inhibit $M$. tuberculosis growth in vitro as well as in vivo. Its activity was comparable to rifampicin, the most potent anti-Tb drug. Although it 
inhibits nucleic acid synthesis like rifampicin, it was believed that calanolide A may have a different cellular target to rifampicin [498].

Furthermore, the $\alpha$-hydroxytropolones (NSC18806 and NSC310618) were reported as HIV integrase inhibitors [499]. These inhibitors also have $\mathrm{OH}$ groups in their structures and have comparable activity to NSC133457, identified in this study, which also has an $\mathrm{OH}$ group (Figure 5.18-B). These $\mathrm{OH}$ group patterns can be considered for designing a novel class of dual inhibitors, provided their functional group modifications are flexible to allow $\mathrm{OH}$ group substitutions.

\subsubsection{Description of Inhibitors Identified in this Study}

Over the last decade, new leads have been added to the anti-Tb drug pipeline (Figure 5.20). This became possible due to interaction between several disciplines including $M$. tuberculosis pathology, structural biology and mycobacterial genomics. One of the key ways to identify new leads is to perform SAR on novel anti-mycobacterial hits. After the initial discovery of antimicrobials, the evaluation of the pharmacokinetics and mechanism of action of identified leads are important phases of drug discovery. The inclusion of SAR studies helps to progress the new candidate drugs to the clinical trials stage.

Many RNase H compounds exhibited consistent and similar inhibitory activity against $M$. tuberculosis $\mathrm{H} 37 \mathrm{Ra}$ with an $\mathrm{IC}_{50}$ of $25 \mu \mathrm{M}$ or less e.g. NSC14543, NSC20410 and NSC51535. While structures of these compounds are not related to each other (Figure 5.19), they inhibit HIV RNase H activity in HIV (Table 5.1). One interesting feature of most of the RNase $\mathrm{H}$ inhibitors identified in this study is that they have low molecular weights (Table 5.2) and follow some of impotant Lipinski's rules (section 2.6.8.1). Such leads may offer better pharmacokinetic properties and should be further tested for in vivo efficacy to determine 
whether or not they retain a good anti-infectious activity in animal models. To initiate preliminary investigations, it was deemed appropriate to further investigate these compounds for their sensitivity in $\mathrm{Tb}$ screening models in vitro (Chapter 4). A novel anti-Tb compound NSC402959 was identified, which served as an entry point for the discovery of a new class of M. tuberculosis inhibitors (section 5.1). Since, a biologically active compound should be stable, lipophilic (for improved absorption) and non-toxic to humans. The prioritized inhibitors are NSC112200 (Figure 5.16-C) and NSC133457 (Figure 5.5) based on their antimycobacterial activity. These inhibitors have anti-HIV RNase $\mathrm{H}$ activity but do not inhibit human RNase H activity. These compounds also inhibited M. tuberculosis growth in vitro at $6.25 \mu \mathrm{M}$ and $50 \mu \mathrm{M}$ concentrations, respectively (sections 5.3 .4 and 5.5). In particular, NSC112200 appeared to be specific against mycobacterial RNase HI (Figure 7.14). Other inhibitors include NSC35676 and NSC668394, which need to undergo further experimentation, based on their activity that is unrelated to RNase $\mathrm{H}$ inhibition properties. For example, NSC35676 is also a known inhibitor of glucoseamine-1-phosphate acetyl transferase in $M$. tuberculosis (PubChem database), (MIC $12.5 \mu \mathrm{M}$ in this study). This compound also inhibits aspartyl aminopeptidase in P. falciparum (PubChem database). NSC112200 is not only structurally different from the current anti-Tb drugs but also has a novel target in M. tuberculosis, and thus warrants further investigation (section 6.5).

While previously known HIV RNase $\mathrm{H}$ inhibitors have also shown anti-mycobacterial activity in this study, these compounds share a significant structural homology with the active HIV RNase $\mathrm{H}$ inhibitors reported previously (Figures 5.15-5.20). Given an anti-RNase H activity, anti-mycobacterial activity and less toxicity to mammalian cells, NSC133457 appears to have a comfortable safety margin and holds good potential for further development. 


\subsection{New Anti-Tb Clinical Drug Pipeline}

Koul et al. [500] have listed anti-Tb drugs that are currently in the clinical $\mathrm{Tb}$ pipeline under several phase trials (Figure 5.20). Among all the classes described in this summary, none of the drugs targets mycobacterial RNase H. However, a preliminary analysis of selected inhibitors (identified in this study) indicated specific activity against RNase HI in $M$. tuberculosis (Rv2228c) in an enzyme inhibition assay (section 5.4.3). An example include compound NSC112200 (Figure 7.14) which inhibited mycobacterial RNase HI at $5 \mu \mathrm{M}$ concentration compared to untreated controls (also see Figure 5.10, in which NSC112200 showed positive interaction with mycobacterial RNase HI in silico). A detailed kinetic analysis would be performed next to evaluate its potential to block RNase $\mathrm{H}$ activity in $M$. tuberculosis at nanomolar concentrations. Since inhibitors identified in this study (Table 5.1) targeted RNase HI enzyme in M. tuberculosis, there is a good possibility that a new class of M. tuberculosis inhibitors could be presented for $\mathrm{Tb}$, a disease claiming over a million lives every year [501]. 


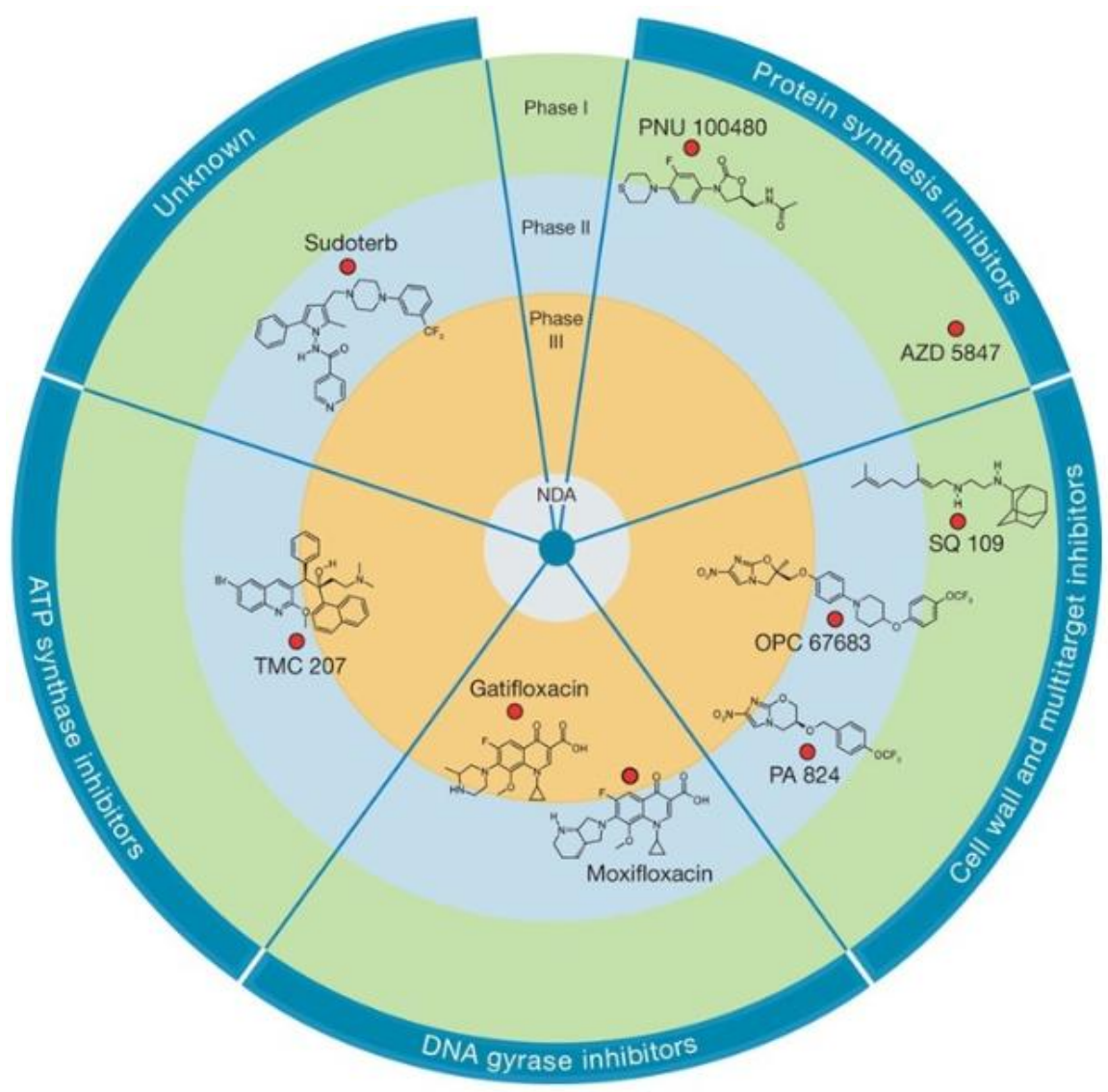

Figure 5.20: Current pipeline of new anti-Tb drugs. Figure was adapted with permission from Koul et al. [500].

Fluoroquinolones, gatifloxacin, and moxifloxacin are expected to reduce the overall treatment time for $\mathrm{Tb}$ due to their improved bactericidal activities compared to current drugs [502] and because they are active against the drug resistant-strains of $\mathrm{Tb}$ [503]. Since the development of drug resistance is inevitable, it can be, at least temporarily, overcome by identifying a novel target in $M$. tuberculosis. This study has identified a number of interesting inhibitors that could be dually targeting HIV and Tb. In particular, NSC402959 exhibited inhibitory activity against $M$. tuberculosis $\mathrm{H} 37 \mathrm{Ra}$ as well as against the virulent $\mathrm{Tb}$ strain, $M$. tuberculosis H37Rv in vitro (Figure 4.12). Since M. tuberculosis H37Ra and M. tuberculosis H37Rv behave almost similarly in drug susceptibility assays [352], it is expected that other RNase $\mathrm{H}$ inhibitors may also cause inhibition of the virulent $M$. tuberculosis strains. An even more effective series of compounds could then be generated through derivatisation and 
appropriate modification of compounds like NSC402959 that are active against $M$. tuberculosis.

Overlapping toxicities in a drug regimen for the treatment of $\mathrm{Tb} / \mathrm{HIV}$ is a serious concern when a separate drug is used for each pathogen. It may be possible to be overcome this by having a single target in both species that allows a monotherapy for the simultaneous treatment of the two different diseases. RNase $\mathrm{H}$ inhibitors could therefore be developed and tested in an animal model to generate in vivo chemotherapy data in order to completely evaluate their positive therapeutic potential and scale their activity profiles as well as their potential to be developed as a novel class of inhibitors for the simultaneous treatment of $\mathrm{Tb}$ and HIV.

Finally, previously known RNase $\mathrm{H}$ inhibitors of HIV were tested against $M$. tuberculosis in the present study to evaluate their in vitro inhibition against mycobacterial species. New RNase $\mathrm{H}$ compounds have been identified that have good anti-mycobacterial activity (Table 5.2). Although a biochemical assay did confirm the specificity of HIV RNase H inhibitors at a preliminary scale, however, a detailed kinetic study will be required to scale up their activity against $M$. tuberculosis RNase $\mathrm{HI}$ and for further development of dual inhibitors of HIV and $\mathrm{Tb}$ RNase $\mathrm{H}$. Novel compounds that are active against these dual infections and are also active against resistant strains would be preferred for subsequent clinical benefit.

\subsection{Conclusion}

In conclusion, this study has identified a small number of new RNase $\mathrm{H}$ inhibitors of $M$. tuberculosis (Table 5.2), such as NSC112200 and NSC133457 that show promise for further development as anti-Tb drugs. Since these compounds are known HIV RNase H inhibitors, they could be developed even further to dually target HIV and M. tuberculosis infections and 
thus may have implications for a combined $\mathrm{Tb}$ and AIDS therapy. To the best of our knowledge, these anti-HIV compounds together with their anti-mycobacterial properties have not been described before. 
Chapter 6

Summary and Future Directions 


\subsection{Research Summary}

The main focus and research rationale of this study was to discover new inhibitors of $M$. tuberculosis and to examine the possibility of identifying a novel class of inhibitor(s) from selected compound libraries that have the potential to be developed into anti-Tb drugs. A number of in vitro $\mathrm{Tb}$ models have been used for anti-Tb drug discovery, but no reports are available on which $\mathrm{Tb}$ model is best and most selective in drug screening programmes. This research identified $M$. bovis $\mathrm{BCG}$ as a more sensitive in vitro $\mathrm{Tb}$ model than previously used M. smegmatis. $M$. bovis BCG was considerably more selective in identifying inhibitors that were active against $M$. tuberculosis. A number of interesting mycobacterial inhibitors have been identified in this study during drug screening which could be pursued for further development in future research. NSC402959, a novel anti-mycobacterial compound identified from the NIH library and had an MIC value between $6.25 \mu \mathrm{M}-12.5 \mu \mathrm{M}$ against $M$. tuberculosis. It not only exhibited significant inhibitory activity against the virulent strains of Tb but also demonstrated low toxicity in mammalian cells.

The complete genome sequence of $M$. tuberculosis has helped researchers to develop more efficient diagnostic tools for $\mathrm{Tb}$ [356]. However, an in-depth knowledge is still required in persistent $M$. tuberculosis infection to understand the latent form of the disease. Antimycobacterial compounds identified in this study were also tested in dormicidal assays to evaluate their ability to kill the persistent $M$. tuberculosis (non-replicating) cells from over four week old cultures. NSC402959 was not found to be dormicidal against both $M$. tuberculosis $\mathrm{H} 37 \mathrm{Ra}$ and $M$. tuberculosis $\mathrm{H} 37 \mathrm{Rv}$ compared to positive drug controls.

The next aim of this study was to identify a dual in vitro inhibitor of Tb/HIV. Known HIV anti-RNase $\mathrm{H}$ inhibitors were retrieved from the NIH library and tested against $M$. tuberculosis for their anti-mycobacterial activity in dose-response assays. NSC133457 is an 
HIV RNase H inhibitor and does not interfere with the human enzyme (PubChem database), it was identified as a mycobacterial inhibitor in this study ( $\left.\mathrm{IC}_{50} 25 \mu \mathrm{M}\right)$. It also did not exhibit toxicity against HL-60 cells. Despite structural differences between HIV and M. tuberculosis RNase HI proteins, in silico analysis performed in this study supported the binding of novel RNase H ligands NSC35676, NSC668394 and NSC112200 with M. tuberculosis RNase HI protein (Figure 5.10 and Table 5.4). Furthermore, a cell free assay confirmed the specific anti-RNase H activity of NSC112200 (Figure 7.14) and opened the door for the identification of a novel class of $M$. tuberculosis inhibitors that also inhibit HIV replication in vitro. RNase $\mathrm{H}$ enzyme appears to be essential to the life cycle of both $M$. tuberculosis and HIV. It is therefore possible that a dual inhibitor against both species can be developed that could modify the available treatment regimen and benefit dually-infected patients.

A better understanding of the dual biology and a positive drug-drug interaction is the key to containing HIV/Tb co-infections [504]. If these compounds retain decent inhibitory activity against $M$. tuberculosis in vitro after performing the required chemical modifications, this research will be extended to in vivo assays to ascertain whether these agents work in infected animal models (section 6.5). Successful treatment outcome may be possible by inhibiting both HIV and Tb through a single-target dual inhibitor for these two pathogens. Dual inhibitory activity of compounds against $\mathrm{HIV}$ and $\mathrm{Tb}$ identified in this study make them attractive candidates for further investigation to demonstrate their clinical benefits.

\subsection{Overall Scientific Contribution of this Study}

- This research highlights the importance of selecting in vitro screens in PC2 laboratories where facilities for screening against virulent $\mathrm{Tb}$ species are not available.

- This study has demonstrated better screening ability of $M$. bovis BCG than $M$. smegmatis as a high throughput screening model for anti-Tb drug discovery. It has 
also identified New Zealand plant species as a good source of novel anti-tubercular lead compounds [241].

- This work has identified a novel inhibitor of M. tuberculosis H37Rv (NSC402959) that has minimum toxicity towards mammalian cell lines and may hold potential as a lead anti-Tb agent [505].

- Furthermore, previously-known anti-HIV inhibitors of RNase H were selected in this study and compounds were identified that also possessed anti-mycobacterial activity in vitro i.e. NSC35676, NSC112200 and NSC133457. In silico analysis was performed to dock interesting HIV RNase $\mathrm{H}$ inhibitors on mycobacterial RNase HI (Rv2228c) to analyse if these inhibitors also possess better binding energies and have specificity for RNase HI.

- Given the specific activity of NSC112200 against Rv2228c in an enzyme inhibition assay, RNase $\mathrm{HI}$ has been identified as a novel drug target in M. tuberculosis.

- With in vitro data against M. tuberculosis and positive results from docking studies, this part of research defines a specific future prospect of generating better derivatives of compounds to be developed into a novel class of M. tuberculosis inhibitors. It also has opened a new window that may eventually lead to the development of dual inhibitors of $\mathrm{Tb}$ and HIV.

\subsection{Future Prospects for This Research}

\subsubsection{Opportunistic Pathogens in Tb-HIV Co-infection}

Patients living with AIDS and Tb are vulnerable to various bacterial and viral pathogens that can cause respiratory problems (section 5.1). One common example is Chlamydia pneumoniae that causes pneumonia [506]. Other HIV opportunistic pathogens include cytomegalo virus (CMV) [507] and herpes virus [508]. 


\subsubsection{A Triad Inhibitor against Major Human Killers}

Infectious diseases have been a major human health concern for decades and more than 15 million associated deaths are recorded due to infections from HIV, Tb and malaria [509]. Apart from the concerns about Tb and HIV co-infection [510], malaria alone appears to claim more than 2.5 million lives each year and is one the growing epidemics in the world today. Novel inhibitors are therefore also required for P. falciparum, the malarial parasite vector, as development of drug resistance to the available drug therapy is inevitable [511-515]. It is therefore necessary to develop novel and more effective drugs against all three of these infections. One way of containing such combined infections is to develop a single-target inter-kingdom inhibitor that could potentially inhibit the above three species (Figure 6.1). This combined pill would reduce the daily pill burden, improving compliance and reducing complexity of treatment regimens. Identifying inhibitor(s) that block the replication of $M$. tuberculosis and the life cycles of HIV and $P$. falciparum is plausible, since functional RNase H genes are present in M. tuberculosis [420], HIV [458] and P. falciparum [516]. Similarly TETD (Disulfiram) has previously been shown to inhibit the glycolytic pathway in $P$. falciparum [517]. Given the glycolytic inhibitory activity of TETD against $M$. tuberculosis as well, a co-inhibition of both infections could be achieved simultaneously by using a single inhibitor. Although, the cellular target of TETD in M. tuberculosis is not known but it inhibited the growth of $M$. tuberculosis H37Ra and M. bovis BCG in this study (Table 3.4). Other examples of drugs that may show multiple activities are aklavine $\mathrm{HCl}$ and mitoxantrone, that were both characterized as anti-mycobacterial compounds in this study (Table 3.5) and had previously been shown to inhibit $P$. falciparum growth as well (section

$$
\text { 3.3.1.2.4). }
$$

Furthermore, during compound screening in this project, NSC668394 was identified as a mycobacterial inhibitor with an in vitro $\mathrm{MIC}$ value against $M$. tuberculosis of $1.56 \mu \mathrm{M}$ (this 
study) from the NIH chemical library. It also has previously been shown to have activity against HIV and P. falciparum (PubChem). Given its low MIC against M. tuberculosis and the fact that RNase HI is present and essential in these species, it may be possible to develop a triad inhibitor (section 6.3.3), which is not only active across these species but may also be effective against the suit of opportunistic infections of HIV/AIDS (Figure 6.1). Further experimental studies in vivo therefore would be needed to see if this is achievable.

\subsubsection{An Ultimate Goal for Future Research}

The goal of this research would be to provide a means to minimise some of the problems that can occur when HIV and common opportunistic diseases, such as Tb, candidosis and pneumonia, are treated simultaneously. By targeting a single enzyme that is common to HIV and co-infecting pathogens, toxic and interference activities that can occur between different drugs may be reduced. This new class of drug would offer important benefits to patients with HIV and other related diseases by reducing side effects and improving treatment success rates. Since RNase $\mathrm{H}$ is present in most bacterial, viral and fungal species, including major parasites (section 6.3.2) and is essential to their life cycles, it may be possible to develop an inhibitor common to both HIV and Tb that is also active against a range of other pathogens which only emerge under conditions of severe immuno-deficiency as occur in AIDS. 


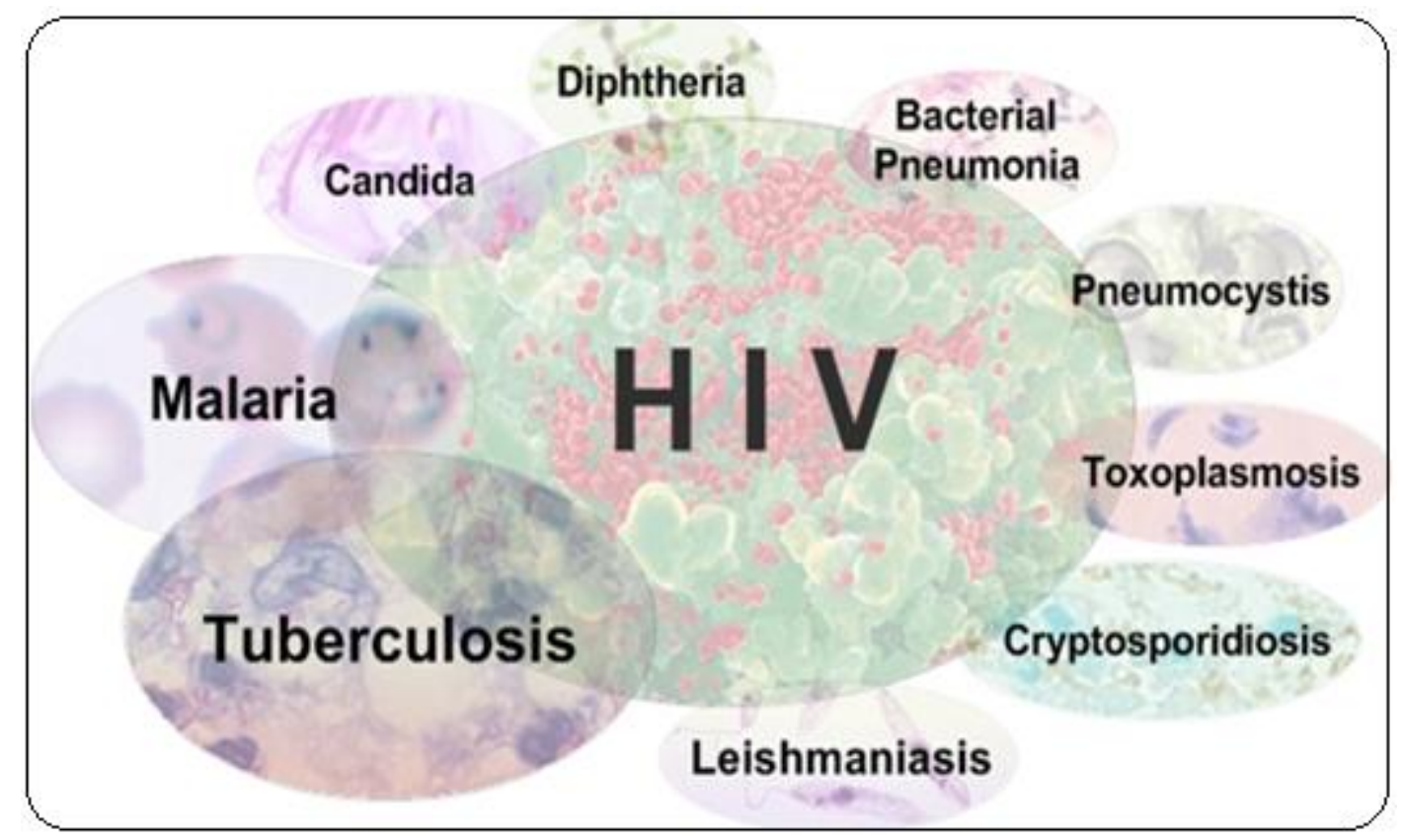

Figure 6.1: Future prospects for the development of an inter-kingdom, single-target RNase HI inhibitor against bacterial, viral, and fungal species for the simultaneous inhibition of $\mathrm{Tb}, \mathrm{HIV}$ and their opportunistic infections.

\subsection{Possible Problems with Successful Drug Development}

In vitro active inhibitors may not be active in vivo as hypothesised [343]; therefore, inhibitors identified in this study (though active against virulent strains in vitro) must be tested in a mouse models to completely evaluate their potential and efficacy in vivo.

Although some of the inhibitors were highly active against M. tuberculosis in vitro and also have activity against clinical strains (S. aureus) e.g. NSC668394 (Chapter 5). Some of the inhibitors also appeared to be active in a range of other assays suggesting that such inhibitors may have multiple targets including human enzymes. A broad activity profile makes these compounds less important in the drug-discovery process. Another possibility, however, is to make them effective against a single target by performing derivatisation to generate single-target active analogues (section 6.5). 


\subsection{Future Research}

A number of interesting mycobacterial inhibitors were discovered in this work. Some of these inhibitors also possess known anti-HIV RNase $\mathrm{H}$ activity and require further experimentation in vivo. Derivatisation of important inhibitors could be performed to generate more effective compounds that could potentially achieve a dual inhibition of HIV and M. tuberculosis. Selected compounds will then be tested in an infection assay [518], to evaluate their intracellular anti-M. tuberculosis efficacy. While induction of $M$. tuberculosis-infectedmacrophages and pneumocytes apoptosis is known, it is not clear whether $M$. tuberculosis drives cellular necrosis or if the apoptosis represents a host immune strategy to halt $M$. tuberculosis replication [519-520].

An RNase HI knockout mutant will be generated to validate this protein as a viable target in M. tuberculosis. This should help defining a link between simultaneous inhibition of both HIV and Mtb RNase H. Finally, inhibitors with ideal pharmacophores will be tested for in vivo efficacy. While no model mimics the disease condition in humans [239], animal models of infection are only expected to be predictive of the infection state [521]. AgResearch, Animal Research Centre, Wallaceville, New Zealand has an established facility for in vivo studies using a validated guinea pig model, which could be used to evaluate potential in vivo activity of anti-Tb compounds identified in this study. Inhibitors that can target interacellular M. tuberculosis, will greatly aid reducing bacterial load and clearing infection as well as in disease eradication. 
References 


\section{References}

1. Hopwell, P.C., Clinical Overview of Tuberculosis, in Tuberculosis: Pathogenesis, Protection, and Control. (Ed., Barry. R. Bloom.). . American Society for Microbiology, 1994: p. 25-46.

2. World Health Organization (1993). Tuberculosis: A Global Emergency. World Health Forum. 14(4): p. p438.

3. World Health Organization (2009). Global Tuberculosis Control: Surveillance, Planning, Financing. World health Organization, Geneva, Switzerland.

4. Dye, C. and B.G. Williams, The Population Dynamics and Control of Tuberculosis. Science, 2010. 328(5980): p. 856-861.

5. Krauss, H., Schiefer, H. G., Weber, A., Slenczka, W., Appel, M., von Graevenitz, A., Enders, B., Zahner, H., Isenberg, H. D., Bacterial Zoonoses. In H. Krauss, H. G. Schiefer, A. Weber, W. Slenczka, M. Appel, A. von Graevenitz, B. Enders, H. Zahner \& H. D. Isenberg (Eds.), Zoonoses: Infectious Diseases Transmissible from Animals to Humans (3rd Ed.) Washington, D.C.: ASM Press, 2003: p. pp. 216-217.

6. Singhaniya, S.B., S.R. Barpande, and J.D. Bhavthankar, Oral tuberculosis in an asymptomatic pulmonary tuberculosis. Oral Surgery, Oral Medicine, Oral Pathology, Oral Radiology, and Endodontology, 2011. 111(3): p. e8-e10.

7. Jacques, J., Sloan, J. M, The changing pattern of miliary tuberculosis. Thorax, 1970. 25: p. 237-240.

8. Huseby JS, H.L., Miliary tuberculosis and adult respiratory distress syndrome Annals of Internal Medicine 1976. 85(5): p. 609-611.

9. Murray HW, T.C., Kirmani N, Sheagren JN., The adult respiratory distress syndrome associated with miliary tuberculosis Chest, 1978. 73(1): p. 37-43.

10. Lee, K., et al., Acute respiratory distress syndrome caused by miliary tuberculosis: a multicentre survey in South Korea. The International Journal of Tuberculosis and Lung Disease, 2011. 15(8): p. 1099-1103.

11. Zumla, A., et al., The tuberculosis pandemic: implications for health in the tropics. Transactions of the Royal Society of Tropical Medicine and Hygiene. 93(2): p. 113117.

12. Mario C, R., The TB epidemic from 1992 to 2002. Tuberculosis, 2003. 83(1-3): p. 414. 
13. Das, R.K., Tuberculosis--historical landmarks. Journal of Indian Medical Association, 2000. 98(3): p. 112-4.

14. Haddad, G.E., Medicine and the Culture of Commemoration: Representing Robert Koch's Discovery of the Tubercle Bacillus. Osiris, 1999. 14: p. 118-137.

15. Kaufmann, S.H.E., U.E. Schaible, 100th anniversary of Robert Koch's Nobel Prize for the discovery of the tubercle bacillus. Trends in Microbiology, 2005. 13(10): p. 469475.

16. Sakula, A., Robert Koch: centenary of the discovery of the tubercle bacillus, 1882. Thorax, 1982. 37(4): p. 246-251.

17. Cole, S.T., et al., Deciphering the biology of Mycobacterium tuberculosis from the complete genome sequence. Nature, 1998. 393(6685): p. 537-544.

18. Camus, J.-C., et al., Re-annotation of the genome sequence of Mycobacterium tuberculosis H37Rv. Microbiology, 2002. 148(10): p. 2967-2973.

19. Gill, W.P., et al., A replication clock for Mycobacterium tuberculosis. Nat Med, 2009. 15(2): p. 211-214.

20. Riley, R.L., et al., Aerial Dissemination of Pulmonary Tuberculosis. A Two-year Study of Contagion in a Tuberculosis Ward. American Journal of Epidemiology, 1959. 70(2): p. 185-196.

21. Wells, W.F., Airborne Contagion and Air Hygiene. Harvard Univ Press, 1955: p. Cambridge, Mass.

22. Pfyffer, G.E., Mycobacterium: general characteristics, laboratory detection, and staining procedures. In P. R. Murray (Ed.). Manual of Clinical Microbiology: Washington D.C., ASM Press, 2006. 1: p. 543-572.

23. David. H. Dail, S.P.H., Philip. T. Cagle, Dail and Hammar's pulmonary pathology, Non-neoplastic Lung Disease. Ch. 9. Tuberculosis and Non-tuberculous Mycobacterial Infections (Ed. by Joseph. F. Tomashefski, Jr. and Carol. F. Farver). 3rd Ed., 2008. 01: p. 316-348.

24. Philippe Herman, M.F.-D., Didier Breyer, Bernadette Van Vaerenbergh, Katia Pauwels, Chuong Dai Do Thi, M. Sneyers, Maryse Wanlin, René Snacken \& William Moens, Biosafety Recommendations for the Contained Use of Mycobacterium tuberculosis Complex Isolates in Industrialized Countries. Royal Library of Belgium Deposit Number : D/2006/2505/22, 2006: p. p-06. 
25. Kimman, T.G., E. Smit, and M.R. Klein, Evidence-Based Biosafety: a Review of the Principles and Effectiveness of Microbiological Containment Measures. Clin. Microbiol. Rev., 2008. 21(3): p. 403-425.

26. W. Emmett Barkley, G.P.K., Biological Safety in the Experimental Tuberculosis Laboratory. Tuberculosis: Pathogenesis, Protection, and Control (Ch.5, Ed. by Barry. R. Bloom). American Society for Microbiology, 1994: p. 65.

27. Delogu, G. and G. Fadda, The quest for a new vaccine against tuberculosis. Journal of Infection in Developing Countries, 2009. 3(1): p. 5-15.

28. American Thoracic Society/Centers for Disease Control and Prevention (2000). Targeted tuberculin testing and treatment of latent tuberculosis infection: Available at http://www.cdc.gov/mmwr/pdf/rr/rr4906.pdf. MMWR 49(RR-06): p. 1-54.

29. Normal chest X-ray: PASIEKA/Science photo library, P590/0211. Available at www. sciencephoto.com. Accessed on December 2011. Science Photo Library.

30. Allen Carolyn M, A.-J.H.H., Irion Klaus L, Al Ghanem Sarah, Gouda Alaa, Khan Ali Nawaz Imaging lung manifestations of HIV/AIDS. Annals of Thoracic Medicine., 2010. 5(4): p. p- 201-216

31. Brosch, R., et al., A new evolutionary scenario for the Mycobacterium tuberculosis complex. Proceedings of the National Academy of Sciences, 2002. 99(6): p. 36843689.

32. Brennan, P.J., Structure of Mycobacteria: Recent Developments in Defining Cell Wall Carbohydrates and Proteins. Reviews of Infectious Diseases, 1989. 11(Suppl. 2 ): p. S420-S430.

33. Segal, W., Growth dynamics of in vivo and in vitro grown mycobacterial pathogens. In: The Mycobacteria: A Sourcebook (Eds. G. P. Kubica, L. G. Wayne. Marcel Dekker) New York, NY 1. 1984: p. 547-73.

34. Hybiske, K. and R.S. Stephens, Exit strategies of intracellular pathogens. Nat Rev Micro, 2008. 6(2): p. 99-110.

35. Meena, L.S. and Rajni, Survival mechanisms of pathogenic Mycobacterium tuberculosis H37Rv. FEBS Journal, 2010. 277(11): p. 2416-2427.

36. Behr, M.A. and D.R. Sherman, Mycobacterial virulence and specialized secretion: same story, different ending. Nat Med, 2007. 13(3): p. 286-287.

37. Hunter, R.L., Pathology of post primary tuberculosis of the lung: An illustrated critical review. Tuberculosis, 2011. 91(6): p. 497-509. 
38. Wayne LG, K.G., The Mycobacteria: (Ed. by) Sneath P. H. A, Mair N. S, Sharpe M. E. Bergey's Manual of Systematic Bacteriology. Williams and Wilkins, 1984. 02: p. 1435 - 1457.

39. Ákos Somoskövi, J.E.H., Marie Fitzgerald, Dianne O’Donnell, Linda M. Parsons, Max Salfinger, Lessons From a Proficiency Testing Event for Acid-Fast Microscopy. Chest, 2001. 120(1): p. 250-257.

40. Ehrlich, P., Referate aus den Verein fur innere Medicin zu Berlin. Deutsche Med Wochenschr, 1882. 8: p. 269.

41. Ziehl, F., Ueber die Fairbung des Tuberkelbacillus. Deutsche Med Wochenschr, 1883. 9: p. 247-249.

42. Neelsen, F., Ein Casuistischer Beitrag zur Lehre von der Tuberkulose. Zentrablau fpr die medizinischen Wissenschafen, 1883. 21: p. 497-501.

43. Daffé. M, D.P., The envelope layers of mycobacteria with reference to their pathogenicity. Adv Microb Physiol., 1998. 39: p. p131-203.

44. Clifton. Barry. E 3rd, L., Richard E, Mdluli, Khisimusi, Sampson, Andrea E, Schroeder, Benjamin G Slayden, Richard A. Yuan Ying, Mycolic acids: structure, biosynthesis and physiological functions. Progress in Lipid Research. 37(2-3): p. 143179.

45. Best. M, S.S.A., Springthorpe. V. S, Kennedy. M. E., Efficacies of selected disinfectants against Mycobacterium tuberculosis. Journal of Clinical Microbiology, 1990. 28(10): p. 2234-2239.

46. Michael Lauzardo, J.R., Mycobacterial Disinfection. In Seymour. S. Block (Ed.): Disinfection, Sterilization, and Preservation. Philadelphia P.A. Lipincott Williams and Wilkins., 1996: p. pp. 513-528.

47. Lind, A., et al., A carrier method for the assessment of the effectiveness of disinfectants against Mycobacterium tuberculosis. Journal of Hospital Infection, 1986. 7(1): p. 60-67.

48. Rutala, W.A., APIC guideline for selection and use of disinfectants. American Journal of Infection Control, 1996. 24(4): p. 313-342.

49. Dorhoi, A., S.T. Reece, and S.H.E. Kaufmann, For better or for worse: the immune response against Mycobacterium tuberculosis balances pathology and protection. Immunological Reviews, 2011. 240(1): p. 235-251. 
50. Glickman MS, J.W., Microbial pathogensis of Mycobacterium tuberculosis: Dawn of a discipline. Cell, 2001. 104: p. p477-485.

51. Chan, J., et al., Lipoarabinomannan, a possible virulence factor involved in persistence of Mycobacterium tuberculosis within macrophages. Infection and Immunity, 1991. 59(5): p. 1755-1761.

52. Schlesinger, L., et al., Differences in mannose receptor-mediated uptake of lipoarabinomannan from virulent and attenuated strains of Mycobacterium tuberculosis by human macrophages. The Journal of Immunology, 1996. 157(10): p. 4568-4575.

53. Delphi Chatterjee, S.W.H., Michael McNeil, and Patrick. J. Brennan, Lipoarabinomannan. Multiglycosylated form of the mycobacterial mannosylphosphatidylinositols. J Biol Chem., 1992. 267(9): p. 6228-6233.

54. Krishnan, N., et al., Mycobacterium tuberculosis Lineage Influences Innate Immune Response and Virulence and Is Associated with Distinct Cell Envelope Lipid Profiles. PLoS ONE, 2011. 6(9): p. e23870.

55. Gagneux, S. and P.M. Small, Global phylogeography of Mycobacterium tuberculosis and implications for tuberculosis product development. The Lancet Infectious Diseases, 2007. 7(5): p. 328-337.

56. Garnier, T., et al., The complete genome sequence of Mycobacterium bovis. Proceedings of the National Academy of Sciences, 2003. 100(13): p. 7877-7882.

57. Daniel, T.M., The impact of tuberculosis on civilization. Infectious Disease Clinics of North America, 2004. 18(1).

58. Ruwen Jou, W.-L.H., Chen-Yuan Chiang, Human Tuberculosis Caused by Mycobacterium bovis, Taiwan. Emerging Infectious Diseases, 2008. 14(3): p. 515-17.

59. Grange, J.M., Mycobacterium bovis infection in human beings. Tuberculosis, 2001. 81(1-2): p. 71-77.

60. Ricardo, d.1.R.-D., Human Mycobacterium bovis infection in the United Kingdom: Incidence, risks, control measures and review of the zoonotic aspects of bovine tuberculosis. Tuberculosis, 2006. 86(2): p. 77-109.

61. Oettinger, T., et al., Development of the Mycobacterium bovis BCG vaccine: review of the historical and biochemical evidence for a genealogical tree. Tubercle and Lung Disease, 1999. 79(4): p. 243-250. 
62. Mahairas, G.G., Sabo, P J., Hickey, M J., Singh, D C., Stover, C K, Molecular analysis of genetic differences between Mycobacterium bovis BCG and virulent M. bovis. Journal of Bacteriology, 1996. 178(5): p. 1274-82.

63. Pym, A.S., et al., Loss of RD1 contributed to the attenuation of the live tuberculosis vaccines Mycobacterium bovis BCG and Mycobacterium microti. Molecular Microbiology, 2002. 46(3): p. 709-717.

64. Castillo-Rodal, A.I., et al., Mycobacterium bovis BCG Substrains Confer Different Levels of Protection against Mycobacterium tuberculosis Infection in a BALB/C Model of Progressive Pulmonary Tuberculosis. Infection and Immunity, 2006. 74(3): p. $1718-1724$.

65. Lin, M.Y., et al., Lack of Immune Responses to Mycobacterium tuberculosis DosR Regulon Proteins following Mycobacterium bovis BCG Vaccination. Infection and Immunity, 2007. 75(7): p. 3523-3530.

66. Colditz GA, B.C., Mosteller F, Brewer TF, Wilson ME, Burdick E, Fineberg HV, The efficacy of bacillus Calmette-Guérin vaccination of newborns and infants in the prevention of tuberculosis: meta-analyses of the published literature. Pediatrics., 1995. 96: p. 29-35.

67. Rodrigues LC, D.V.K., Wheeler J.G., Protective Effect of BCG against Tuberculous Meningitis and Miliary Tuberculosis: A Meta-Analysis. International Journal of Epidemiology, 1993. 22(6): p. 1154-1158.

68. Karonga Prevention Trial Group (1996). Randomised controlled trial of single BCG, repeated BCG, or combined BCG and killed Mycobacterium leprae vaccine for prevention of leprosy and tuberculosis in Malawi. The Lancet. 348(9019): p. 17-24.

69. Barry. R. Bloom, P.E.M.F., The BCG experience: implications for future vaccines against tuberculosis. In Tuberculosis: Pathogenesis, Protection, and Control. American Society for Microbiology, Washington, D.C., 1994: p. p. 531-537.

70. Colditz, G.A., et al., Efficacy of BCG Vaccine in the Prevention of Tuberculosis. JAMA: The Journal of the American Medical Association, 1994. 271(9): p. 698-702.

71. Fine, P.E.M., Variation in protection by BCG: implications of and for heterologous immunity. The Lancet, 1995. 346(8986): p. 1339-1345.

72. Paul E. M. Fine, I.A.M.C., Julie B. Milstien, C. John Clements, Issues relating to the use of BCG in immunization programmes. A discussion document. World Health Organization, Geneva, 1999. 
73. McShane, H., Tuberculosis vaccines: beyond bacille Calmette-Guérin. Philosophical Transactions of the Royal Society B: Biological Sciences, 2011. 366(1579): p. 27822789.

74. Hesseling, A.C., et al., The risk of disseminated Bacille Calmette-Guerin (BCG) disease in HIV-infected children. Vaccine, 2007. 25(1): p. 14-18.

75. van der Wel, N., et al., M. tuberculosis and M. leprae Translocate from the Phagolysosome to the Cytosol in Myeloid Cells. Cell, 2007. 129(7): p. 1287-1298.

76. van Dissel, J.T., et al., Ag85B-ESAT-6 adjuvanted with IC31® promotes strong and long-lived Mycobacterium tuberculosis specific $T$ cell responses in nä̈ve human volunteers. Vaccine, 2010. 28(20): p. 3571-3581.

77. Dietrich, J., et al., Exchanging ESAT6 with TB10.4 in an Ag85B Fusion MoleculeBased Tuberculosis Subunit Vaccine: Efficient Protection and ESAT6-Based Sensitive Monitoring of Vaccine Efficacy. The Journal of Immunology, 2005. 174(10): p. 63326339.

78. Abel, B., et al., The Novel Tuberculosis Vaccine, AERAS-402, Induces Robust and Polyfunctional CD4+ and CD8+ T Cells in Adults. Am. J. Respir. Crit. Care Med., 2010. 181(12): p. 1407-1417.

79. von Reyn, C.F., et al., Prevention of tuberculosis in Bacille Calmette-Guerin-primed, $H I V$-infected adults boosted with an inactivated whole-cell mycobacterial vaccine. AIDS, 2010. 24(5): p. 675-685.

80. Sreevatsan, S., et al., Restricted structural gene polymorphism in the Mycobacterium tuberculosis complex indicates evolutionarily recent globaldissemination. Proceedings of the National Academy of Sciences, 1997. 94(18): p. 9869-9874.

81. Fleischmann, R.D., et al., Whole-Genome Comparison of Mycobacterium tuberculosis Clinical and Laboratory Strains. J. Bacteriol., 2002. 184(19): p. 5479-5490.

82. Baril, L., et al., Tuberculosis Caused by Mycobacterium africanum Associated with Involvement of the Upper and Lower Respiratory Tract, Skin, and Mucosa. Clinical Infectious Diseases, 1995. 21(3): p. 653-655.

83. Meyer, C.G., et al., Pulmonary tuberculosis: Virulence of Mycobacterium africanum and relevance in HIV co-infection. Tuberculosis, 2008. 88(5): p. 482-489.

84. Hugo L. David, M.-T.J., Arlette Jumin, Janine Grandry, Egil H. Lehman, Numerical taxonomy analysis of Mycobacterium africanum. 1978. 28(4): p. 467-472. 
85. de Jong, B.C., M. Antonio, and S. Gagneux, Mycobacterium africanum - Review of an Important Cause of Human Tuberculosis in West Africa. PLoS Negl Trop Dis, 2010. 4(9): p. e744.

86. Cole, S.T., et al., Massive gene decay in the leprosy bacillus. Nature, 2001. 409(6823): p. 1007-1011.

87. Browne, S.G., Some aspects of the history of leprosy: the leprosie of yesterday. Proc R Soc Med., 1975. 68(8): p. 485-493.

88. Sansonetti P, L.P.H., The immunology of leprosy: speculations on the leprosy spectrum. Rev Infect Dis, 1981. 3(03): p. 422-69.

89. Martinez, T.S., et al., Oral mucosa as a source of Mycobacterium leprae infection and transmission, and implications of bacterial DNA detection and the immunological status. Clinical Microbiology and Infection, 2011. 17(11): p. 1653-1658.

90. D. Beyene, A.A., M. Harboe, D. Kidane, M. MacDonald, P. R. Klatser, G. A. Bjune and W. C. S. Smith., Nasal carriage of Mycobacterium leprae DNA in healthy individuals in Lega Robi village, Ethiopia. Epidemiology and Infection, 2003. 131(02): p. pp 841-848.

91. Desikan K, V. and Sreevatsa, Extended studies on the viability of Mycobacterium leprae outside the human body. Leprosy review, 1995. 66(4): p. 287-295.

92. van Beers, S.M., M.Y.L. de Wit, and P.R. Klatser, The epidemiology of Mycobacterium leprae: Recent insight. FEMS Microbiology Letters, 1996. 136(3): p. 221-230.

93. Hershkovitz, I., et al., Detection and Molecular Characterization of 9000-Year-Old Mycobacterium tuberculosis from a Neolithic Settlement in the Eastern Mediterranean. PLoS ONE, 2008. 3(10): p. e3426.

94. Rodrigo Gay Ducati, A.R.-N., Luiz Augusto Basso, D.S. Santos, The Resumption of Consumption : A Review on Tuberculosis. . Mem Inst Oswaldo Cruz, Rio de Janeiro, 2006. 101(7): p. 697-714.

95. Bedino, J.H., Mycobacterium tuberculosis: An In-depth Discussion for Embalmers. The Champion Company, Springfield, 1999. Part 3(No. 638): p. 2566-2569.

96. Atkinson, T.P., M.F. Balish, and K.B. Waites, Epidemiology, clinical manifestations, pathogenesis and laboratory detection of Mycoplasma pneumoniae infections. FEMS Microbiology Reviews, 2008. 32(6): p. 956-973.

97. Herzog, B.H., History of Tuberculosis. Respiration, 1998. 65(1): p. 5-15. 
98. F. Haas, H.S.S., The origins of M. tuberculosis and the notion of its contagiousness. In: Tuberculosis. Eds. Rom W N, Garay S. Little, Brown \& Co., Boston, MA, 1996: p. 3-19.

99. Dixie. E. Snider, M.R., Arata Kochi, Tuberculosis: Pathogenesis, Protection, and Control. Chap1. Global Burden of Tuberculosis (Ed. , Barry. R. Bloom.). . American Society for Microbiology, 1994: p. p47-59.

100. World Health Organisation. Global Tuberculosis Control: WHO progress report 2011 , available

$a t$ http://www.who.int/tb/publications/global_report/2011/gtbr11_full.pdf.

101. Kochi, A., The global tuberculosis situation and the new control strategy of the World Health Organization. Tubercle, 1991. 76: p. p1-6.

102. National Institutes of Health (2010): Fact Sheet - Tuberculosis. Availabe at http://report.nih.gov/nihfactsheets/Pdfs/Tuberculosis(NIAID).pdf, accessed on November 2011.

103. Comstock, G., Epidemiology of Tuberculosis. The American review of respiratory disease, 1982. 125(3): p. 8-15.

104. World Health Organisation. Global Tuberculosis Control: WHO progress report 2010 available

at http://whqlibdoc.who.int/publications/2010/9789241564069 eng.pdf. Accessed on December 2011.

105. Dye, C., et al., Targets for global tuberculosis control. The International Journal of Tuberculosis and Lung Disease, 2006. 10(4): p. 460-462.

106. E. Vynnycky, M.W.B., C. C. Leung, C. M. Tam, P. E. M. Fine, Limited impact of tuberculosis control in Hong Kong: attributable to high risks of reactivation disease. . Epidemiology and Infection, 2008. 136(7): p. 943-952.

107. Stop TB Partnership and World Health Organization (2006). Global Plan to Stop TB 2006-2015. WHO/HTM/STB/2006.35. Geneva, Switzerland: World Health Organization.

108. World Health Organization. Global Tuberculosis Control: Surveillance, Planning, Financing. [WHO Reports 1997, 1999, 2003, 2005, 2007, 2009 and 2011]. Geneva: WHO; 1997-2011.

109. Kaufmann, S.H.E., How can immunology contribute to the control of tuberculosis? Nat Rev Immunol, 2001. 1(1): p. 20-30. 
110. Stewart, G.R., B.D. Robertson, and D.B. Young, Tuberculosis: a problem with persistence. Nat Rev Micro, 2003. 1(2): p. 97-105.

111. Dye, C., et al., Global Burden of Tuberculosis. JAMA: The Journal of the American Medical Association, 1999. 282(7): p. 677-686.

112. de Chastellier, C., The many niches and strategies used by pathogenic mycobacteria for survival within host macrophages. Immunobiology, 2009. 214(7): p. 526-542.

113. Yanmin, H., and Anthony, R. M. Coates, Acute and Persistent Mycobacterium tuberculosis Infections Depend on the Thiol Peroxidase TPX. PLoS ONE, 2009. 4(4): p. e5150.

114. Rustad, T.R., et al., Hypoxia: a window into Mycobacterium tuberculosis latency. Cellular Microbiology, 2009. 11(8): p. 1151-1159.

115. Cosma, C.L., D.R. Sherman, and L. Ramakrishnan, The Secret Lives of the Pathogenic Mycobacteria. Annual Review of Microbiology, 2003. 57(1): p. 641-676.

116. Blanchard, J.S., Molecular Mechanisms of Drug Resistance in Mycobacterium Tuberculosis. Annual Review of Biochemistry, 1996. 65(1): p. 215-239.

117. Barry, C.E., et al., The spectrum of latent tuberculosis: rethinking the biology and intervention strategies. Nat Rev Micro, 2009. 7(12): p. 845-855.

118. Manabe, Y.C., Bishai, W. R, Latent Mycobacterium tuberculosis-persistence, patience, and winning by waiting. Nature Medicine, 2000. 6: p. 1327-1329.

119. Bigger, J.W., Treatment of Staphylococcal Infections with Penicillin. The Lancet, 1944. Vol. 2: p. p. 497-500.

120. Keren, I., et al., Persister cells and tolerance to antimicrobials. FEMS Microbiology Letters, 2004. 230(1): p. 13-18.

121. Parrish, N.M., J.D. Dick, and W.R. Bishai, Mechanisms of latency in Mycobacterium tuberculosis. Trends in Microbiology, 1998. 6(3): p. 107-112.

122. Wayne, L.G. and L.G. Hayes, An in vitro model for sequential study of shiftdown of Mycobacterium tuberculosis through two stages of nonreplicating persistence. Infection and Immunity, 1996. 64(6): p. 2062-9.

123. Wayne, L.G. and C.D. Sohaskey, Nonreplicating persistence of Mycobacterium tuberculosis. Annual Review of Microbiology, 2001. 55(1): p. 139-163.

124. Dick, T., B.H. Lee, and B. Murugasu-Oei, Oxygen depletion induced dormancy in Mycobacterium smegmatis. FEMS Microbiology Letters, 1998. 163(2): p. 159-164. 
125. Wayne, L.G., Synchronized replication of Mycobacterium tuberculosis. Infection and Immunity, 1977. 17(3): p. 528-530.

126. Wayne, L.G., Dormancy of Mycobacterium tuberculosis and latency of disease. European Journal of Clinical Microbiology and Infectious Diseases, 1994. 13(11): p. 908-14.

127. Wayne, L. and L. Hayes, An in vitro model for sequential study of shiftdown of Mycobacterium tuberculosis through two stages of nonreplicating persistence. Infect. Immun., 1996. 64(6): p. 2062-2069.

128. Chao, M.C. and E.J. Rubin, Letting Sleeping dos Lie: Does Dormancy Play a Role in Tuberculosis? Annual Review of Microbiology, 2010. 64(1): p. 293-311.

129. Dick, T., Dormant tubercle bacilli: the key to more effective TB chemotherapy? Journal of Antimicrobial Chemotherapy, 2001. 47(1): p. 117-118.

130. Zumla, A., et al., Viewpoint: Scientific dogmas, paradoxes and mysteries of latent Mycobacterium tuberculosis infection. Tropical Medicine \& International Health, 2011. 16(1): p. 79-83.

131. Stover, C.K., et al., A small-molecule nitroimidazopyran drug candidate for the treatment of tuberculosis. Nature, 2000. 405(6789): p. 962-966.

132. Sekosan M, C.M., Senseng C, Farolan M, Sekosan J., Spindle cell pseudotumors in the lungs due to Mycobacterium tuberculosis in a transplant patient. The American Journal of Surgical Pathology, 1994. 18(10): p. 1065-8.

133. Joe Philip, M.B.B., Sakshi Dua Mycobacterial Spindle Cell Pseudotumor of the Lung. Chest, 2012. 142(3): p. 783-784.

134. Baron, K.M., Aranda, C. P, Diagnosis of mediastinal mycobacterial lymphadenopathy by transbronchial needle aspiration. Chest, 1991. 100(6): p. 1723-4.

135. Logani S, L.D., Cheng JD, Ioachim HL, Adsay NV, Spindle cell tumors associated with mycobacteria in lymph nodes of HIV-positive patients: Kaposi sarcoma with mycobacteria' and 'mycobacterial pseudotumor. The American Journal of Surgical Pathology, 1999. 23(6): p. 656-61.

136. MOH., Guidelines for Tuberculosis Control in New Zealand 2003. Wellington: Ministry of Health. 2002.

137. Turnbull, F., The Epidemiology and Surveillance of Tuberculosis in New Zealand. Ministry of Health, New Zealand. 2003. 
138. Ministry of Health. Guidelines for Tuberculosis Control in New Zealand 2010. Available at http://www.moh.govt.nz. Accessed on December 2011. Ministry of Health, Wellington.

139. Ange Bissielo., E.L., Helen Heffernan, Tuberculosis in New Zealand: Annual Report 2010. Institute of Environmental Science and Research Ltd. , 2011: p. New Zealand.

140. Das D, B.M., Calder L., Tuberculosis epidemiology in New Zealand: 1995-2004. The New Zealand Medical Journal (NZMA), 2006. 119(1243).

141. Mark Thomas, R.E.-P., Tuberculosis in New Zealand: poverty casts a long shadow. The New Zealand Medical Journal (NZMA), 2006. 119(1243).

142. Tze Liang Goh, C.R.T., Katharine L Jones, Joshua T Freeman and Colin S Wong, Extensively drug-resistant tuberculosis: New Zealand's first case and the challenges of management in a low-prevalence country. The Medical Journal of Australia (MJA), 2011. 194(11): p. 602-604.

143. World Health Organisation (2010). MDR/XDR-Tb: Global Report on Surveillance and Response 2010.

144. Raviglione, M.C. and M.W. Uplekar, WHO's new Stop TB Strategy. The Lancet. 367(9514): p. 952-955.

145. Viveiros, M., et al., Direct Application of the INNO-LiPA Rif.TB Line-Probe Assay for Rapid Identification of Mycobacterium tuberculosis Complex Strains and Detection of Rifampin Resistance in 360 Smear-Positive Respiratory Specimens from an Area of High Incidence of Multidrug-Resistant Tuberculosis. Journal of Clinical Microbiology, 2005. 43(9): p. 4880-4884.

146. Rose DN, S.C., Adler JJ., Interpretation of the tuberculin skin test. Journal of General Internal Medicine 1995. 10(11): p. 635-42.

147. Menzies, D., What Does Tuberculin Reactivity after Bacille Calmette-Guérin Vaccination Tell Us? Clinical Infectious Diseases, 2000. 31(Supplement 3): p. S71S74.

148. Ramam, M., et al., How useful is the Mantoux test in the diagnosis of doubtful cases of cutaneous tuberculosis? International Journal of Dermatology, 2011. 50(11): p. 1379-1382.

149. Arend, S.M., et al., Detection of Active Tuberculosis Infection by T Cell Responses to Early-Secreted Antigenic Target 6-kDa Protein and Culture Filtrate Protein 10. Journal of Infectious Diseases, 2000. 181(5): p. 1850-1854. 
150. Amin, I., et al., PCR could be a method of choice for identification of both pulmonary and extra-pulmonary tuberculosis. BMC Research Notes, 2011. 4(1): p. 332.

151. Nopvichai, C., et al., PCR detection of Mycobacterium tuberculosis in necrotising non-granulomatous lymphadenitis using formalin-fixed paraffin-embedded tissue: a study in Thai patients. Journal of Clinical Pathology, 2009. 62(9): p. 812-815.

152. Daniel, T.M., The rapid diagnosis of tuberculosis: a selective review. Journal of Laboratory and Clinical Medicine 1990. 116(3): p. 277-82.

153. Su, W.J., Recent advances in the molecular diagnosis of tuberculosis. Journal of Microbiology, Immunology and Infection 2002. 35(4): p. 209-14.

154. Tenover, F.C., Rapid Detection and Identification of Bacterial Pathogens Using Novel Molecular Technologies: Infection Control and Beyond. Clinical Infectious Diseases, 2007. 44(3): p. 418-423.

155. Deepti Parashar, C.D., Sharma VD, Katoch VM., Applications of real-time PCR technology to mycobacterial research. Indian Journal of Medical Research 2006. 124(4): p. 385-98.

156. Soysal, A., et al., Diagnosing latent tuberculosis infection in haemodialysis patients: T-cell based assay (T-SPOT.TB) or tuberculin skin test? Nephrology Dialysis Transplantation, 2012. 27(4): p. 1645-1650.

157. F Kakkar, U.A., D Ling, M Pai, IC Kitai, Tuberculosis in children: New diagnostic blood tests. Journal of Paediatrics and Child Health 2010. 15(8): p. 529-533.

158. Davis. P.D.O, Tuberculosis Diagnosis, in International Encyclopedia of Public Health, H. Editor-in-Chief: Kris, Editor. 2008, Academic Press: Oxford. p. 371-381.

159. Kent PT, K.G., Public health mycobacteriology : a guide for the level III laboratory. 1985, U.S. Dept. of Health and Human Services, Public Health Service, Centers for Disease Control: Atlanta, Ga. :.

160. Salfinger M, P.G., The new diagnostic mycobacteriology laboratory. European Journal of Microbiology and Infectious Diseases, 1994. 13(11): p. 961-79.

161. Warren, J., Bhattacharya M, Kleper N.F. De Almeida, Trakas K, Peterson LR, A Minimum $5.0 \mathrm{ml}$ of Sputum Improves the Sensitivity of Acid-fast Smear for Mycobacterium tuberculosis. American Journal of Respiratory and Critical Care Medicine, 2000. 161(5): p. 1559-1562.

162. J M Grange, J.L.S., Dogma and innovation in the global control of tuberculosis: discussion paper. J R Soc Med., 1994. 87(5): p. 272-275. 
163. Rook, G.A.W. and R. Hernandez-Pando, The Pathogenesis of Tuberculosis. Annual Review of Microbiology, 1996. 50(1): p. 259-284.

164. Sterling, T.R., et al., The Scope and Impact of Treatment of Latent Tuberculosis Infection in the United States and Canada. American Journal of Respiratory and Critical Care Medicine, 2006. 173(8): p. 927-931.

165. Campbell, I.A. and O. Bah-Sow, Pulmonary tuberculosis: diagnosis and treatment. BMJ, 2006. 332(7551): p. 1194-1197.

166. World Health Organization. Treatment of tuberculosis: Guidelines for National Programmes. Geneva: WHO, 2003. (WHO/CDS/TB/2003.313). Available at http://whqlibdoc.who.int/hq/2003/WHO_CDS_TB_2003.313_eng.pdf. .

167. Juan Ruiz-Manzano, R.B., José Luis Calpe, José A. Caminero, Joan Caylà, José A. Domínguez, José María García, Rafael Vidal, Diagnosis and Treatment of Tuberculosis. . Arch Bronconeumol., 2008. 44(10): p. 551-66.

168. Zhang, Y., The Magic Bullets and tuberculosis Drug Targets. Annual Review of Pharmacology and Toxicology, 2005. 45(1): p. 529-564.

169. Wehrli W, K.F., Schmid K, Staehelin M, Interaction of rifamycin with bacterial RNA polymerase. Proceedings of the National Academy of Sciences (USA), 1968. 61(2): p. 667-73.

170. Vilchèze, C., et al., Transfer of a point mutation in Mycobacterium tuberculosis inhA resolves the target of isoniazid. Nature Medicine, 2006. 12(9): p. 1027-1029.

171. Shi, W., et al., Pyrazinamide Inhibits Trans-Translation in Mycobacterium tuberculosis. Science, 2011. 333(6049): p. 1630-1632.

172. L Deng, K.M., K G Robuck, M Scherman, P J Brennan, M R McNeil, Recognition of multiple effects of ethambutol on metabolism of mycobacterial cell envelope. Antimicrobial Agents and Chemotherapy, 1995. 39(3): p. 694-701.

173. Chan ED, C.D., Iseman MD, Heifets LB, Pyrazinamide, Ethambutol, Ethionamide, and Aminoglycosides. In: Rom WN, Garay SM. Tuberculosis, 2nd edition. Philadelphia, PA: Lippincott Williams \& Wilkins, 2003: p. 773789.

174. Fu, L.M. and T.M. Shinnick, Genome-wide exploration of the drug action of capreomycin on Mycobacterium tuberculosis using Affymetrix oligonucleotide GeneChips. Journal of Infection, 2007. 54(3): p. 277-284. 
175. Banerjee A, D.E., Quemard A, Balasubramanian V, Um KS, Wilson T, Collins D, de Lisle G, Jacobs WR Jr, inhA, a gene encoding a target for isoniazid and ethionamide in Mycobacterium tuberculosis. Science, 1994. 263(5144): p. 227-30.

176. Johnsson, K., D. S. King, and P. G. Schultz, Studies on the mechanism of action of isoniazid and ethionamide in the chemotherapy of tuberculosis. Journal of the American Chemical Society 1995. 117: p. 5009-10.

177. Engohang-Ndong, J., et al., EthR, a repressor of the TetR/CamR family implicated in ethionamide resistance in mycobacteria, octamerizes cooperatively on its operator. Molecular Microbiology, 2004. 51(1): p. 175-188.

178. Brennan PJ, Y.D.B., Handbook of Anti-Tuberculosis Agents: Global Alliance for TB Drug Development. Tuberculosis, 2008. 88(2): p. 85-170.

179. Gillespie, S.H., Evolution of Drug Resistance in Mycobacterium tuberculosis: Clinical and Molecular Perspective. Antimicrob. Agents Chemother., 2002. 46(2): p. 267-274.

180. Skeiky, Y.A.W. and J.C. Sadoff, Advances in tuberculosis vaccine strategies. Nat Rev Micro, 2006. 4(6): p. 469-476.

181. Dye, C., et al., Prospects for worldwide tuberculosis control under the WHO DOTS strategy. The Lancet, 1998. 352(9144): p. 1886-1891.

182. Centers for Disease Control and Prevention. Tuberculosis Treatment, available at http://www.cdc.gov/tb/topic/treatment/default.htm accessed on November 2011.

183. Gordin, F., Chaisson, R. E., Matts, J. P., et al.,, Rifampin and pyrazinamide vs isoniazid for prevention of tuberculosis in HIV-infected persons: An international randomized trial. The Journal of the American Medical Association, 2000. 283(11): p. 1445-1450.

184. International Union Against Tuberculosis Committee on Prophylaxis. Efficacy of various durations of isoniazid preventive therapy for tuberculosis: five years of follow-up in the IUAT trial. Bulletin of the World Health Organisation 1982. 60(4): p. 555-564.

185. Whalen, C.C., Johnson, John L., Okwera, Alphonse., Hom, David L., Huebner, Robin., Mugyenyi, Peter., Mugerwa, Roy D., Ellner, Jerrold J., Nsubuga, Peter., Vjecha, Michael., Myanja, Harriet., Kityo, Cissy., Loughlin, Anita., Milberg, John., Pekovic, Vukosava, A Trial of Three Regimens to Prevent Tuberculosis in Ugandan 
Adults Infected with the Human Immunodeficiency Virus. New England Journal of Medicine, 1997. 337(12): p. 801-808.

186. Yee, D., et al., Incidence of Serious Side Effects from First-Line Antituberculosis Drugs among Patients Treated for Active Tuberculosis. American Journal of Respiratory and Critical Care Medicine, 2003. 167(11): p. 1472-1477.

187. Schaberg T, R.K., Lode H., Risk factors for side-effects of isoniazid, rifampin and pyrazinamide in patients hospitalized for pulmonary tuberculosis. Eur Respir J., 1996. 9(10): p. 2026-30.

188. Agal, S., et al., Monitoring and management of antituberculosis drug induced hepatotoxicity. Journal of Gastroenterology and Hepatology, 2005. 20(11): p. 17451752.

189. Kopanoff, D.E., Snider, D. E Jr., Caras, G. J., Isoniazid-related hepatitis: a U.S. Public Health Service cooperative surveillance study. The American Review of Respiratory Disease 1978. 117(6): p. 991-1001.

190. Franks, A.L., Binkin, N. J., Snider, D. E. Jr., Rokaw, W. M and Becker. S, Isoniazid hepatitis among pregnant and postpartum Hispanic patients. Public Health Reports, 1989. 104(2): p. 151-155.

191. Snider, D.E. and G.J. Caras, Isoniazid-associated Hepatitis Deaths: A Review of Available Information. American Journal of Respiratory and Critical Care Medicine, 1992. 145(2 Pt 1): p. 494-497.

192. Nolan, C.M., Goldberg, S. V., and Buskin, S. E., Hepatotoxicity associated with isoniazid preventive therapy: A 7-year survey from a public health tuberculosis clinic. The Journal of the American Medical Association, 1999. 281(11): p. 1014-1018.

193. American Thoracic Society/Centers for Disease Control and Prevention/Infectious Diseases Society of America, joint statement: Treatment of Tuberculosis. American Journal of Respiratory and Critical Care Medicine, 2003. 167(4): p. 603-662.

194. Andrews, J.R., et al., Predictors of Multidrug- and Extensively Drug-Resistant Tuberculosis in a High HIV Prevalence Community. PLoS ONE, 2010. 5(12): p. e15735.

195. Andrews, J.R., et al., Multidrug-Resistant and Extensively Drug-Resistant Tuberculosis: Implications for the HIV Epidemic and Antiretroviral Therapy Rollout in South Africa. Journal of Infectious Diseases, 2007. 196(Supplement 3): p. S482S490. 
196. Velayati, A.A., et al., Emergence of New Forms of Totally Drug-Resistant Tuberculosis Bacilli. Chest, 2009. 136(2): p. 420-425.

197. Baylan, O., Extensively drug resistant and extremely drug resistant tuberculosis forms after multi-drug resistant tuberculosis: new faces of the old disease. Mikrobiyol Bul., 2011. 45(1): p. 181-95.

198. Jean Leandro dos Santos, C.M.C., Ednir de Oliveira Vizioli, Fernando Rogério Pavan and Luiz Antonio Dutra, Health Interventions to Improve the Medication Efficacy in Tuberculosis Treatment: In, Understanding Tuberculosis - Global Experiences and Innovative Approaches to the Diagnosis (Ed., by Pere-Joan Cardona). Janeza Trdine, InTech, 2012(Ch. 25): p. 527-552.

199. Castro, K.G., Tuberculosis Surveillance: Data for Decision-Making. Clinical Infectious Diseases, 2007. 44(10): p. 1268-1270.

200. Julie S. Philalay, C.O.P., Kirsten A. Hauge, Tige R. Rustad, and Gerard A. Cangelosi, Genes Required for Intrinsic Multidrug Resistance in Mycobacterium avium. Antimicrobial Agents and Chemotherapy, 2004. 48(9): p. 3412-3418.

201. Viveiros, M., C. Leandro, and L. Amaral, Mycobacterial efflux pumps and chemotherapeutic implications. International Journal of Antimicrobial Agents, 2003. 22(3): p. 274-278.

202. Telenti, A., Genetics of drug resistant tuberculosis. Thorax, 1998. 53(9): p. 793-797.

203. Schürch, A.C., et al., The tempo and mode of molecular evolution of Mycobacterium tuberculosis at patient-to-patient scale. Infection, Genetics and Evolution, 2010. 10(1): p. 108-114.

204. Wehrli, W., et al., Interaction of Rifamycin with Bacterial RNA Polymerase. Proceedings of the National Academy of Sciences of the United States of America, 1968. 61(2): p. 667-673.

205. Giovanni Di Perri, S.B., Which agents should we use for the treatment of multidrugresistant Mycobacterium tuberculosis? Journal of Antimicrobial Chemotherapy, 2004. 54(3): p. 593-602.

206. Ramaswamy, S.V., et al., Molecular Genetic Analysis of Nucleotide Polymorphisms Associated with Ethambutol Resistance in Human Isolates of Mycobacterium tuberculosis. Antimicrob. Agents Chemother., 2000. 44(2): p. 326-336.

207. Belanger, A.E., et al., The embAB Genes of Mycobacterium avium Encode an Arabinosyl Transferase Involved in Cell Wall Arabinan Biosynthesis that is the Target 
for the Antimycobacterial Drug Ethambutol. Proc Natl Acad Sci USA 1996. 93(21): p. 11919-11924.

208. Goude, R., et al., The Arabinosyltransferase EmbC Is Inhibited by Ethambutol in Mycobacterium tuberculosis. Antimicrobial Agents and Chemotherapy, 2009. 53(10): p. 4138-4146.

209. Scorpio, A., et al., Characterization of pncA mutations in pyrazinamide-resistant Mycobacterium tuberculosis. Antimicrob. Agents Chemother., 1997. 41(3): p. 540543.

210. Davies, A.P., et al., Comparison of Phenotypic and Genotypic Methods for Pyrazinamide Susceptibility Testing with Mycobacterium tuberculosis. J. Clin. Microbiol., 2000. 38(10): p. 3686-3688.

211. Lewis, K., Persister cells, dormancy and infectious disease. Nat Rev Micro, 2007. 5(1): p. 48-56.

212. Zignol, M., et al., Global Incidence of Multidrug-Resistant Tuberculosis. Journal of Infectious Diseases, 2006. 194(4): p. 479-485.

213. Shao, Y., et al., Epidemiology of anti-tuberculosis drug resistance in a chinese population: current situation and challenges ahead. BMC Public Health, 2011. 11(1): p. 110.

214. Mdivani, N., et al., High prevalence of multidrug-resistant tuberculosis in Georgia. International Journal of Infectious Diseases, 2008. 12(6): p. 635-644.

215. Aziz, M.A., et al., Epidemiology of antituberculosis drug resistance (the Global Project on Anti-tuberculosis Drug Resistance Surveillance): an updated analysis. The Lancet, 2006. 368(9553): p. 2142-2154.

216. Virginia, G., The threat of multidrug resistance: is tuberculosis ever untreatable or uncontrollable? The Lancet, 1999. 353(9157): p. 998-999.

217. Su WJ, F.J., Huang CC, Perng RP, Increasing Drug Resistance of Mycobacterium tuberculosis Isolates in a Medical Center in Northern Taiwan. Journal of the Formosan Medical Association, 2008. 107(3): p. 259-264.

218. Jagannath, C., V.M. Reddy, and P.R.J. Gangadharam, Enhancement of drug susceptibility of multi-drug resistant strains of Mycobacterium tuberculosis by ethambutol and dimethyl sulphoxide. Journal of Antimicrobial Chemotherapy, 1995. 35(3): p. 381-390. 
219. Robert C. Goldman, K.V.P., Barbara E. Laughon The Evolution of Extensively Drug Resistant Tuberculosis (XDR-TB): History, Status and Issues for Global Control Infectious Disorders - Drug Targets, 2007. 7(2): p. 73-91.

220. N. Sarita Shah, A.W., Gill-Han Bai, Lucia Barrera, Fadila Boulahbal, Nuria MartínCasabona, Francis Drobniewski, Chris Gilpin, Marta Havelková, Rosario Lepe, Richard Lumb, Beverly Metchock, Françoise Portaels, Maria Filomena Rodrigues, Sabine Rüsch-Gerdes, Armand Van Deun, Veronique Vincent, Kayla Laserson, Charles Wells, and J. Peter Cegielski, Worldwide Emergence of Extensively Drugresistant Tuberculosis. Emerg Infect Dis, 2007. 13(3): p. 380-387.

221. XDR-TB - a global threat. The Lancet 2006. 368(9540): p. p964.

222. Sonnenberg, P., et al., Tuberculosis treatment failure and drug resistance-same strain or reinfection? Transactions of the Royal Society of Tropical Medicine and Hygiene, 2000. 94(6): p. 603-607.

223. World Health Organisation: Tuberculosis, Global Facts 2010-2011.

224. Sun, Z., et al., Characterization of Extensively Drug-Resistant Mycobacterium tuberculosis Clinical Isolates in China. J. Clin. Microbiol., 2008: p. JCM.00822-08.

225. Cohen, J., Extensively Drug-Resistant TB Gets Foothold in South Africa. Infectious Disease, 2006. 313(5793): p. p-1554.

226. Dukes Hamilton, C., et al., Extensively Drug-Resistant Tuberculosis: Are We Learning from History or Repeating It? Clinical Infectious Diseases, 2007. 45(3): p. 338-342.

227. Dheda, K., et al., Extensively Drug-resistant Tuberculosis: Epidemiology and Management Challenges. Infectious Disease Clinics of North America, 2010. 24(3): p. $705-725$.

228. Lumb R, B.I., Carter R, Jelfs P, Keehner T, Sievers A, Tuberculosis in Australia: bacteriologically confirmed cases and drug resistance, 2007. A report of the Australian Mycobacterium Reference Laboratory Network. Commun Dis Intell, 2009. 33(3): p. 298-303.

229. Branch, C.D., Communicable Diseases Report, NSW, September and October 2010. New South Wales Public Health Bulletin, 2011. 21(12): p. 274-279.

230. Muayad Merza, M.R.M., Extensively drug-resistant tuberculosis (XDR) and extremely drug-resistant tuberculosis (XXDR): risk factors and molecular perspectives. Iranian Journal of Clinical Infectious Diseases, 2010. 5(3): p. 174-188. 
231. Paul E Alexander, P.D., The emergence of extensively drug-resistant tuberculosis (TB): TB/HIV coinfection, multidrug-resistant $T B$ and the resulting public health threat from extensively drug-resistant TB, globally and in Canada. Canadian Journal of Infectious Diseases and Medical Microbiology, 2007. 18(5): p. 289-91.

232. Gandhi, N.R., et al., Extensively drug-resistant tuberculosis as a cause of death in patients co-infected with tuberculosis and HIV in a rural area of South Africa. The Lancet. 368(9547): p. 1575-1580.

233. Wallace, R.J., B.A. Brown, and D.E. Griffith, Nosocomial Outbreaks/Pseudo Outbreaks Caused by Nontuberculous Mycobacteria. Annual Review of Microbiology, 1998. 52(1): p. 453-490.

234. Siddiqui UA, O.T.M., Kabir Z, Qureshi S, Gibbons N, Kane M, Keane J, Smoking prolongs the infectivity of patients with tuberculosis. Ir Med J., 2010. 103(9): p. p. 278-80.

235. Lonnroth, K., et al., Alcohol use as a risk factor for tuberculosis - a systematic review. BMC Public Health, 2008. 8(1): p. 289.

236. Foreword., C.J., In: Davies PDO, ed. Clinical Tuberculosis. London: Chapman \& Hall Medical 1994. 1994: p. xv-xvi.

237. Ann M, G., Tuberculosis drug development: Progress, challenges, and the road ahead. Tuberculosis, 2010. 90(3): p. 162-167.

238. Ma, Z., et al., Global tuberculosis drug development pipeline: the need and the reality. The Lancet, 2010. 375(9731): p. 2100-2109.

239. Ronan, O.T., Chapter 3 - Experimental Models Used to Study Human Tuberculosis, in Advances in Applied Microbiology, I.L. Allen, S. Sima, and M.G. Geoffrey, Editors. 2010, Academic Press. p. 75-89.

240. Hanahan, D., Technics for transformation of E. coli, in DNA cloning. A practical approach (Glover, D. M., ed.) IRL Press, Oxford, Washington DC, 1985. 1: p. 109136,.

241. Earl, E., et al., Native New Zealand plants with inhibitory activity towards Mycobacterium tuberculosis. BMC Complementary and Alternative Medicine, 2010. 10(1): p. 25.

242. Snapper, S.B., et al., Isolation and characterization of efficient plasmid transformation mutants of Mycobacterium smegmatis. Molecular Microbiology, 1990. 4(11): p. 1911-1919. 
243. Luo, Y., et al., A novel method for monitoring Mycobacterium bovis BCG trafficking with recombinant $B C G$ expressing green fluorescent protein. Clinical and Diagnostic Laboratory Immunology, 1996. 3(6): p. 761-8.

244. Wang S.Y., Z.H.J., Lv L.D., Wang B.F., Zhang X.L., Pu S.Y., Zhu G.F., Wang H.H., Zhao G.P., Zhang Y, Complete genomic sequence of Mycobacterium tuberculosis strain H37Ra, a non-pathogenic variant closely related to the well-characterized pathogenic strain H37Rv. Genbank, 2007.

245. Steenken. W. Jr, W.H.O., Jr., S. A. Petroff, Biological Studies of the Tubercle Bacillus III. Dissociation and Pathogenicity of the $R$ and $S$ Variants of the Human Tubercle Bacillus (H37). J Exp Med., 1934. 60(4): p. 515-540.

246. Bourn, W.R., et al., Creation and characterisation of a high-copy-number version of the pAL5000 mycobacterial replicon. Tuberculosis, 2007. 87(6): p. 481-488.

247. Dempsey, S.G., Developing a Cell Based Screen for Inhibitors of Two Component Signal Transduction in Mycobacteria. MS Thesis. SBS, Victoria University, Wellington, 2009(print).

248. Guilhot, C., et al., Efficient transposition in mycobacteria: construction of Mycobacterium smegmatis insertional mutant libraries. Journal of Bacteriology, 1994. 176(2): p. 535-539.

249. Wayne, L.J., Cultivation of Mycobacterium tuberculosis for Research Purposes, In: Tuberculosis: Pathogenesis, Protection, and Control. (Ed. , Barry. R. Bloom.). . American Society for Microbiology, 1994: p. 73-83.

250. Earl, E.A., Antibacterial effects of New Zealand plant extracts against mycobacteria. MS Thesis, Victoria University of Wellington, New Zealand. 2010.

251. Lipinski, C.A., et al., Experimental and computational approaches to estimate solubility and permeability in drug discovery and development settings. Advanced Drug Delivery Reviews, 1997. 23(1-3): p. 3-25.

252. Miller, C.H., et al., Modifying Culture Conditions in Chemical Library Screening Identifies Alternative Inhibitors of Mycobacteria. Antimicrobial Agents and Chemotherapy, 2009. 53(12): p. 5279-5283.

253. Smith, C.F. and D.E. Townsend, A New Medium for Determining the Total Plate Count in Food. Journal of Food Protection, 1999. 62(12): p. 1404-1410. 
254. Palomino, J.-C., et al., Resazurin Microtiter Assay Plate: Simple and Inexpensive Method for Detection of Drug Resistance in Mycobacterium tuberculosis. Antimicrobial Agents and Chemotherapy, 2002. 46(8): p. 2720-2722.

255. Campbell, P.A., B.P. Canono, and D.A. Drevets, Measurement of Bacterial Ingestion and Killing by Macrophages, in Current Protocols in Immunology. 2001, John Wiley \& Sons, Inc.

256. Tanya. Parish., N.S., Mycobacteria Protocols. Humana Press, 2008. Vol. 1.

257. Ducati, R.G., et al., The resumption of consumption : a review on tuberculosis. Memórias do Instituto Oswaldo Cruz, 2006. 101: p. 697-714.

258. Nathional Institute of Allergy and Infectious Diseases (2006). Understanding microbes in sickness and in health $\mathrm{NIoH}$.

259. Gandhi, N.R., et al., Multidrug-resistant and extensively drug-resistant tuberculosis: a threat to global control of tuberculosis. The Lancet, 2010. 375(9728): p. 1830-1843.

260. Senior, K., Call to action against HIV/tuberculosis co-infection. The Lancet Infectious Diseases, 2008. 8(7): p. 409-409.

261. World Health Organization: Tuberculosis; Fact Sheet No104, revised August 2002: Available at http://www.who.int/mediacentre/factsheets/whol04/en/print.html accessed December 2011.

262. Global Alliance for TB Drug Development (2001). Scientific Blueprint for Tuberculosis Drug Development. Tuberculosis. 81 (Suppl. 1): p. p. 1-52.

263. Young, D.B. and K. Duncan, Prospects for New Interventions in the Treatment and Prevention of Mycobacterial Disease. Annual Review of Microbiology, 1995. 49(1): p. 641-673.

264. Sacchettini, J.C., E.J. Rubin, and J.S. Freundlich, Drugs versus bugs: in pursuit of the persistent predator Mycobacterium tuberculosis. Nature Reviews Microbiology, 2008. 6(1): p. 41-52.

265. Ananthan, S., et al., High-throughput screening for inhibitors of Mycobacterium tuberculosis H37Rv. Tuberculosis, 2009. 89(5): p. 334-353.

266. Christophe, T., et al., High Content Screening Identifies Decaprenyl-Phosphoribose 2' Epimerase as a Target for Intracellular Antimycobacterial Inhibitors. PLoS Pathog, 2009. 5(10): p. e1000645. 
267. Lavelin, I., et al., Discovery of Novel Proteasome Inhibitors Using a High-Content Cell-Based Screening System. PLoS ONE, 2009. 4(12): p. e8503.

268. Garcia, E., et al., High-throughput screening of enzyme inhibition using an inhibitor gradient generated in a microchannel. Lab on a Chip, 2007. 7(2): p. 249-255.

269. Hildebert B. Maurice, E.T., Kennedy Mwambete., Virtual high screening throughput and design of 14-lanosterol demethylase inhibitors against Mycobacterium tuberculosis. African Journal of Biotechnology, 2009. 8(13): p. pp. 3072-3078.

270. Lei Ji, Q.L., Dacheng Yang, Jianping Xie, Identification of Mannich Base as a Novel Inhibitor of Mycobacterium tuberculosis Isocitrate by High-Throughput Screening Int J Biol Sci., 2011. 7: p. 376-382

271. Anthony, K.G., et al., New Classes of Alanine Racemase Inhibitors Identified by High-Throughput Screening Show Antimicrobial Activity against Mycobacterium tuberculosis. PLoS ONE, 2011. 6(5): p. e20374.

272. Mahalakshmi Vasan, J.N., Jessica Williams, Daniel J. Wilson, Aaron M. Teitelbaum, Rory P. Remmel, Courtney C. Aldrich, Inhibitors of the Salicylate Synthase (MbtI) from Mycobacterium tuberculosis Discovered by High-throughput Screening. ChemMedChem. , 2010. 5: p. 2079 - 2087.

273. Miesel, L., J. Greene, and T.A. Black., Genetic strategies for antibacterial drug discovery. . Nat Rev Genet., 2003. 4(6): p. 442-456.

274. Galluzzi, L. and M. Karp, Whole Cell Strategies Based on lux Genes for High Throughput Applications Toward New Antimicrobials, in Combinatorial Chemistry \& High Throughput Screening. 2006, Bentham Science Publishers Ltd. p. 501-514.

275. Dömling, A., S. Achatz, and B. Beck, Novel anti-tuberculosis agents from MCR libraries. Bioorganic \&amp; Medicinal Chemistry Letters, 2007. 17(19): p. 54835486.

276. Russell, D.G., C.E. Barry, and J.L. Flynn, Tuberculosis: What We Don't Know Can, and Does, Hurt Us. Science, 2010. 328(5980): p. 852-856.

277. Yi Luo, A.S., Xiaohong Chen, William C. DeWolf and Michael A. Donnell., A novel method for monitoring Mycobacterium bovis BCG trafficking with recombinant BCG expressing green fluorescent protein. Clinical and Diagnostic Laboratory Immunology, 1996. 03(06): p. 761-68. 
278. Hoffner, S., C. Jimenez-Misas, and A. Lundin, Improved extraction and assay of mycobacterial ATP for rapid drug susceptibility testing. Luminescence, 1999. 14(5): p. 255-261.

279. Chung, G.A., et al., High-throughput screen for detecting antimycobacterial agents. Antimicrobial Agents and Chemotherapy, 1995. 39(10): p. 2235-8.

280. Cushion, M.T. and D. Ebbets, Growth and metabolism of Pneumocystis carinii in axenic culture. Journal of Clinical Microbiology, 1990. 28(6): p. 1385-1394.

281. March, J.C., G. Rao, and W.E. Bentley, Biotechnological applications of green fluorescent protein. Applied Microbiology and Biotechnology, 2003. 62(4): p. 303315.

282. Williams, S.L., N.B. Harris, and R.G. Barletta, Development of a Firefly LuciferaseBased Assay for Determining Antimicrobial Susceptibility of Mycobacterium aviumsubsp. paratuberculosis. Journal of Clinical Microbiology, 1999. 37(2): p. 304309.

283. Deb, D.K., et al., Bioluminescent Mycobacterium aurum Expressing Firefly Luciferase for Rapid and High Throughput Screening of Antimycobacterial Drugs in Vitro and in Infected Macrophages. Biochemical and Biophysical Research Communications, 2000. 279(2): p. 457-461.

284. Collins, L.A., M.N. Torrero, and S.G. Franzblau, Green Fluorescent Protein Reporter Microplate Assay for High-Throughput Screening of Compounds against Mycobacterium tuberculosis. Antimicrob. Agents Chemother., 1998. 42(2): p. 344347.

285. Changsen, C., S.G. Franzblau, P. Palittapongarnpim, Improved Green Fluorescent Protein Reporter Gene-Based Microplate Screening for Antituberculosis Compounds by Utilizing an Acetamidase Promoter. . Antimicrob. Agents Chemother., 2003. 47(12): p. 3682-3687.

286. L. A. Collins, M.N.T., S. G. Franzblau, Green Fluorescent Protein Reporter Microplate Assay for High-Throughput Screening of Compounds against Mycobacterium tuberculosis. Antimicrob Agents Chemother., 1998. 42(2): p. 344347.

287. Ahmed A. Zafer, Y.E.T., Syed A. Sattar, Rapid Screening Method for Mycobactericidal Activity of Chemical Germicides That Uses Mycobacterium terrae 
Expressing a Green Fluorescent Protein Gene. Applied and Environmental Microbiology, 2001. 67(3): p. 1239-1245.

288. Shaner, N.C., P.A. Steinbach, and R.Y. Tsien, A guide to choosing fluorescent proteins. Nat Meth, 2005. 2(12): p. 905-909.

289. Library of Pharmacologically Active Compounds from Sigma Aldrich. LOPAC Navigator, avaiable at, http://www.sigmaaldrich.com/sigma-aldrich/areas-ofinterest/chemistry/drug-discovery/validation-libraries/lopac1280.html. Accessed November 2011.

290. Sean T. Byrne, P.G., Jiangbing Zhou, Steven M. Denkin, Curtis Chong, David Sullivan, Jun O. Liu, Ying Zhang, Pyrrolidine Dithiocarbamate and Diethyldithiocarbamate are Active against Growing and Nongrowing Persister Mycobacterium tuberculosis. Antimicrob Agents Chemother., 2007. 51(12): p. 44954497.

291. Chen, C.H., et al., The in vitro activity of beta-lactamase inhibitors in combination with cephalosporins against M. tuberculosis. Proc Natl Sci Counc Repub China B, 1995. 19(2): p. 80-84.

292. Prudhomme, M., et al., Modifications of benzoxazole ring substituents in A. 23187 (calcimycin). Effect on cation carrier properties. Journal of Inclusion Phenomena and Macrocyclic Chemistry, 1987. 5(1): p. 99-102.

293. Perskvist, N., et al., Mycobacterium tuberculosis Promotes Apoptosis in Human Neutrophils by Activating Caspase-3 and Altering Expression of Bax/Bcl-xL Via an Oxygen-Dependent Pathway. The Journal of Immunology, 2002. 168(12): p. 63586365.

294. Gutierrez-Lugo, M.-T., et al., Dequalinium, a New Inhibitor of Mycobacterium tuberculosis Mycothiol Ligase Identified by High-Throughput Screening. Journal of Biomolecular Screening, 2009. 14(6): p. 643-652.

295. Tuberculosis Antimicrobial Acquisition and Coordinating Facility. Available at: www.taacf.org. Accessed on November 2011.

296. García-García, A., et al., Search of Chemical Scaffolds for Novel Antituberculosis Agents. Journal of Biomolecular Screening, 2005. 10(3): p. 206-214.

297. Zgoda-Pols, J.R., et al., Antimicrobial Resveratrol Tetramers from the Stem Bark of Vatica oblongifolia ssp. oblongifolia. Journal of Natural Products, 2002. 65(11): p. 1554-1559. 
298. V. Bartoníček, J.T., Effect of Tetraethyl Thiuram Disulphide (Disulfiram) on Metabolism of Trichloroethylene in Man. Br J Ind Med., 1962. 19(3): p. 216-221.

299. U.S. Environmental Protection Agency (2009). Screening-level Hazard Characterization: Thiuram Category. High Production Volume (HPV): p. p. 1-30.

300. Müller, F., P. Ackermann, and P. Margot, Fungicides, Agricultural, 2. Individual Fungicides, in Ullmann's Encyclopedia of Industrial Chemistry. 2000, Wiley-VCH Verlag GmbH \& Co. KGaA.

301. Burman, W.J., et al., Lack of toxicity from concomitant directly observed disulfiram and isoniazid-containing therapy for active tuberculosis. The International Journal of Tuberculosis and Lung Disease, 2002. 6(9): p. 839-842.

302. Liao Qiang-Qiang, Y.Z.-W., Zhu Zhong-Wei, Wang Yi, Zhang Yu, Zhou Guo-Ding, Zhou Qi. , Corrosion Inhibition Effect of Self-Assembled Monolayers of Ammonium Pyrrolidine Dithiocarbamate on Copper. Acta Phys. Chim. Sin., 2009. 25(8): p. 1655-1661.

303. Kartha, G. and K. Krishnamurthy, Crystal and molecular structure of pyrrolidine dithio carbamate cobalt (III) chelate. Journal of Chemical Sciences, 1983. 92(4): p. 437-441.

304. Elisabeth Gaudernak, J.S., Andrea Triendl, Andreas Grassauer, Ernst Kuechler, Antiviral Effects of Pyrrolidine Dithiocarbamate on Human Rhinoviruses. J Virol., 2002. 76(12): p. 6004-6015.

305. Hoffner SE, O.-L.B., Rydgård KJ, Svenson SB, Källenius G., Susceptibility of Mycobacteria to Fusidic Acid. Eur J Clin Microbiol Infect Dis., 1990. 9(4): p. 294297.

306. Scott G. Franzblau, A.N.B., Eugene B. Harris, Fusidic Acid is Highly Active against Extracellular and Intracellular Mycobacterium leprae. Antimicrobial Agents and Chemotherapy, 1992. 36(1): p. 92-94.

307. Takahashi, E. and T. Beppu, A new nucleosidic antibiotic AT-265. J Antibiot (Tokyo), 1982. 35(8): p. 939-947.

308. Masahiro Koshiba, H.K., Takashi Nakazawa, Nobuhide Hayashi, Ryuichi Saura, Noriko Kitamura, Shunichi Kumagai, 2-Chloroadenosine but not adenosine induces apoptosis in rheumatoid fibroblasts independently of cell surface adenosine receptor signalling. Br J Pharmacol. , 2002. 135(6): p. 1477-1486. 
309. Simeonov, A., et al., Identification and Characterization of Inhibitors of Human Apurinic/apyrimidinic Endonuclease APE1. PLoS ONE, 2009. 4(6): p. e5740.

310. Weisman, J.L., et al., Searching for New Antimalarial Therapeutics amongst Known Drugs. Chemical Biology \& Drug Design, 2006. 67(6): p. 409-416.

311. Poonpilas Hongmanee, K.R., Benjamas Buabut, Boontiwa Somsri, Prasit Palittapongarnpim, In Vitro Activities of Cloxyquin (5-Chloroquinolin-8-ol) against Mycobacterium tuberculosis. Antimicrob Agents Chemother., 2006. 51(3): p. 11051106.

312. LW Scheibel, A.A., W Trager, Tetraethylthiuram disulfide (Antabuse) inhibits the human malaria parasite Plasmodium falciparum. Proc Natl Acad Sci USA., 1979. 76(10): p. 5303-5307.

313. Shin, S.J. and M.T. Collins, Thiopurine Drugs Azathioprine and 6-Mercaptopurine Inhibit Mycobacterium paratuberculosis Growth In Vitro. Antimicrobial Agents and Chemotherapy, 2008. 52(2): p. 418-426.

314. Perry CM, S.L., Cefdinir: A review of its use in the management of mild-to-moderate bacterial infections. Drugs, 2004. 64(13): p. 1433-64.

315. Yip, K.W., et al., Potential use of alexidine dihydrochloride as an apoptosispromoting anticancer agent. Molecular Cancer Therapeutics, 2006. 5(9): p. 22342240.

316. Laurent Kremer, C.d.C., Gary Dobson, Kevin J. C. Gibson, Pablo Bifani, Stéphanie Balor, Jean-Pierre Gorvel, Camille Locht, David E. Minnikin, Gurdyal S. Besra, Identification and structural characterization of an unusual mycobacterial monomeromycolyldiacylglycerol. Molecular Microbiology, 2005. 57(4): p. 11131126.

317. Roy S, M.P., Effect of glutamine analogue-acivicin on tumor induced angiogenesis in Ehrlich ascites carcinoma. Indian J Exp Biol., 2005. 43(5): p. 407-13.

318. Zhang, Y., H. Zhang, and Z. Sun, Susceptibility of Mycobacterium tuberculosis to weak acids. Journal of Antimicrobial Chemotherapy, 2003. 52(1): p. 56-60.

319. Sunita Bansod, M.R., Antifungal Activity of Essential Oils from Indian Medicinal Plants Against Human Pathogenic Aspergillus fumigatus and A. niger. World Journal of Medical Sciences, 2008. 3(2): p. 81-88.

320. Rojas, J., et al., Screening for antimicrobial activity of ten medicinal plants used in Colombian folkloric medicine: A possible alternative in the treatment of non- 
nosocomial infections. BMC Complementary and Alternative Medicine, 2006. 6(1): p. 2.

321. Choi, C.W., et al., Antioxidant activity and free radical scavenging capacity between Korean medicinal plants and flavonoids by assay-guided comparison. Plant Science, 2002. 163(6): p. 1161-1168.

322. Thuong, P.T., et al., Antioxidant activities of coumarins from Korean medicinal plants and their structure-activity relationships. Phytotherapy Research, 2010. 24(1): p. 101-106.

323. Njume C, A.A., Samie A, Ndip RN., Inhibitory and bactericidal potential of crude acetone extracts of Combretum molle (Combretaceae) on drug-resistant strains of Helicobacter pylori. J Health Popul Nutr., 2011. 29(5): p. 438-45.

324. Packer, J., et al., An ethnobotanical study of medicinal plants used by the Yaegl Aboriginal community in northern New South Wales, Australia. Journal of Ethnopharmacology, (0).

325. Brooker SG, C.R., Cooper RC New Zealand Medicinal Plants. $2^{\text {nd }}$ Ed. Auckland Reed Publishing (NZ) Ltd., 1987.

326. Goldie, W.H., Maori Medical Lore. Transactions of the New Zealand Institute. . Auckland Southern Reprints, 1998.

327. Zhang, J.-H., T.D.Y. Chung, and K.R. Oldenburg, A Simple Statistical Parameter for Use in Evaluation and Validation of High Throughput Screening Assays. Journal of Biomolecular Screening, 1999. 4(2): p. 67-73.

328. Baell, J.B. and G.A. Holloway, New Substructure Filters for Removal of Pan Assay Interference Compounds (PAINS) from Screening Libraries and for Their Exclusion in Bioassays. Journal of Medicinal Chemistry, 2010. 53(7): p. 2719-2740.

329. Tuberculosis Drug Screening Program, Search for New Drugs for Treatment of Tuberculosis. Antimicrob. Agents Chemother., 2001. 45(7): p. 1943-1946.

330. Hu Jun, Y.X.a., Wang Wei, Wu Hao, Hua Lei, Du Li jun., Antioxidant Activity In Vitro of Three Constituents from Caesalpinia sappan L. Tsinghua Science and Technology 2008. 13(4): p. 474-479.

331. Hikino, H.T., Takashi; Fujimura, Hajime; Hiramatsu, Yasuzo, The validity of oriental medicine. Planta Med., 1977. 31: p. 214-220.

332. Baek, N.-I., et al., Anticonvulsant compounds from the wood of Caesalpinia sappan L. Archives of Pharmacal Research, 2000. 23(4): p. 344-348. 
333. Sei Ryang Oh, D.S.K., Im Seon Lee, Keun Young Jung, Jung Joon Lee, Hyeong-Kyu Lee, Anticomplementary Activity of Constituents from the Heartwood of Caesalpinia sappan. Planta Medica, 1998. 64(5): p. 456-458.

334. Moon, C.-K., et al., Brazilin Protects Cultured Rat Hepatocytes from BrCCl3-induced Toxicity. Drug Chem Toxicol., 1992. 15(1): p. 81-91.

335. Chung, T.H., et al., Investigation of korean plant extracts for potential phytotherapeutic agents against B-virus hepatitis. Phytotherapy Research, 1995. 9(6): p. 429-434.

336. Kirtikar KR, B.B., Indian Medicinal Plants, Dehradun, India. International Book Distributors, 1987: p. p847.

337. Batubara Irmanida, M.T., Ohashi Hideo Brazilin from Caesalpinia sappan wood as an antiacne agent. Journal of wood science, 2010. 56(1): p. 77-81.

338. Takikawa, H. and K. Suzuki, Modified Chiral Triazolium Salts for Enantioselective Benzoin Cyclization of Enolizable Keto-Aldehydes: Synthesis of (+)-Sappanone B. Organic Letters, 2007. 9(14): p. 2713-2716.

339. Jiaju Zhou, G.X., Xinjian Yan., Molecular Structures, Pharmacological Activities, Natural Sources and Applications. Encyclopedia of Traditional Chinese Medicines. 6: p. p107.

340. Siddaiah, V., et al., Synthesis, stereochemical assignments, and biological activities of homoisoflavonoids. Bioorganic \&amp; Medicinal Chemistry, 2006. 14(8): p. 25452551.

341. Wang, Y.-Z., S.-Q. Sun, and Y.-B. Zhou, Extract of the dried heartwood of Caesalpinia sappan L. attenuates collagen-induced arthritis. Journal of Ethnopharmacology, 2011. 136(1): p. 271-278.

342. Images of Caesalpinia sappan were retrieved from Google images search engine available from https://www.google.co.nz/imghp?hl=en\&tab=ii. Retrieved December 2011.

343. Pethe, K., et al., A chemical genetic screen in Mycobacterium tuberculosis identifies carbon-source-dependent growth inhibitors devoid of in vivo efficacy. Nat Commun, 2010. 1: p. 57.

344. Philips, J.A. and J.D. Ernst, Tuberculosis Pathogenesis and Immunity. Annual Review of Pathology: Mechanisms of Disease, 2011. 7(1): p. p353-384. 
345. Migliori, G.B., et al., Review of multidrug-resistant and extensively drug-resistant TB: global perspectives with a focus on sub-Saharan Africa. Tropical Medicine \& International Health, 2010. 15(9): p. 1052-1066.

346. Borgdorff, M.W. and P.M. Small, Scratching the surface of ignorance on MDR tuberculosis. The Lancet, 2009. 373(9678): p. 1822-1824.

347. Nwaka, S. and R.G. Ridley, Virtual drug discovery and development for neglected diseases through public-private partnerships. Nat Rev Drug Discov, 2003. 2(11): p. 919-928.

348. Nwaka, S. and A. Hudson, Innovative lead discovery strategies for tropical diseases. Nat Rev Drug Discov, 2006. 5(11): p. 941-955.

349. Chirac, P. and E. Torreele, Global framework on essential health R\&amp;D. The Lancet. 367(9522): p. 1560-1561.

350. Casenghi, M., S.T. Cole, and C.F. Nathan, New Approaches to Filling the Gap in Tuberculosis Drug Discovery. PLoS Med, 2007. 4(11): p. e293.

351. Carvalho, R., et al., A High-Throughput Screen for Tuberculosis Progression. PLoS ONE, 2011. 6(2): p. e16779.

352. Collins, L. and S.G. Franzblau, Microplate alamar blue assay versus BACTEC 460 system for high-throughput screening of compounds against Mycobacterium tuberculosis and Mycobacterium avium. Antimicrobial Agents and Chemotherapy, 1997. 41(5): p. 1004-9.

353. Schaller, A., et al., Salicylate Reduces Susceptibility of Mycobacterium tuberculosis to Multiple Antituberculosis Drugs. Antimicrobial Agents and Chemotherapy, 2002. 46(8): p. 2636-2639.

354. Tuberculosis Research and Development (2011). Report on Tuberculosis Research Funding Trends 2005- 2010. Treatment Action Group (2011).

355. Betts, J.C., et al., Comparison of the proteome of Mycobacterium tuberculosis strain H37Rv with clinical isolate CDC 1551. Microbiology, 2000. 146(12): p. 3205-3216.

356. Cole, S.T., Why sequence the genome of Mycobacterium tuberculosis? Tubercle and Lung Disease, 1996. 77(6): p. 486-490.

357. Brosch, R., et al., Genome plasticity of BCG and impact on vaccine efficacy. Proceedings of the National Academy of Sciences, 2007. 104(13): p. 5596-5601. 
358. Danilchanka O, M.C., Niederweis M., Identification of a novel multidrug efflux pump of Mycobacterium tuberculosis. Antimicrobial Agents and Chemotherapy, 2008. 52(7): p. 2503-11.

359. Aranday Cortes, E., et al., Mycobacterium bovis BCG Vaccination Induces Specific Pulmonary Transcriptome Biosignatures in Mice. PLoS ONE, 2010. 5(6): p. e11319.

360. Kazuhiro. Matsuo, Y.Y., Mycobacterium bovis Bacille Calmette-Gu'erin as a Vaccine Vector for Global Infectious Disease Control. Tuberculosis Research and Treatment, 2011. 2011(ID574591): p. p1-9.

361. Ritz N, T.M., Connell TG, Sievers A, Robins-Browne R, Curtis N, Susceptibility of Mycobacterium bovis BCG vaccine strains to antituberculous antibiotics. Antimicrobial Agents and Chemotherapy, 2008. 53(1): p. 316-8.

362. Shishido, Y., et al., Anti-tuberculosis drug susceptibility testing of Mycobacterium bovis BCG Tokyo strain. The International Journal of Tuberculosis and Lung Disease, 2007. 11(12): p. 1334-1338.

363. Rastogi, N., et al., Activity of rifapentine and its metabolite 25-O-desacetylrifapentine compared with rifampicin and rifabutin against Mycobacterium tuberculosis, Mycobacterium africanum, Mycobacterium bovis and M. bovis BCG. Journal of Antimicrobial Chemotherapy, 2000. 46(4): p. 565-570.

364. Imaeda, T., Deoxyribonucleic acid Relatedness Among Selected Strains of Mycobacterium tuberculosis, Mycobacterium bovis, Mycobacterium bovis BCG, Mycobacterium microti and Mycobacterium africanum. International Journal of Systematic Bacteriology, 1985. 35(2): p. 147-150.

365. $\mathrm{R}$ Frothingham, H.G.H., $\mathrm{K} \mathrm{H}$ Wilson, Extensive DNA sequence conservation throughout the Mycobacterium tuberculosis complex. Journal of Clinical Microbiology, 1994. 32(7): p. 1639-43.

366. Lewis, K.N., et al., Deletion of RD1 from Mycobacterium tuberculosis Mimics Bacille Calmette-Guérin Attenuation. Journal of Infectious Diseases, 2003. 187(1): p. 117123.

367. Lee, H.R., et al., Novel Multiplex PCR Using Dual-Priming Oligonucleotides for Detection and Discrimination of the Mycobacterium tuberculosis Complex and M. bovis BCG. Journal of Clinical Microbiology, 2010. 48(12): p. 4612-4614. 
368. Sassetti, C.M. and E.J. Rubin, Genetic requirements for mycobacterial survival during infection. Proceedings of the National Academy of Sciences, 2003. 100(22): p. 12989-12994.

369. Karolína Buriánková, F.D.-P., Olivier Dorson, Anne Gondran, Jean-Claude Ghnassia, Jaroslav Weiser, and Jean-Luc Pernodet, Molecular Basis of Intrinsic Macrolide Resistance in the Mycobacterium tuberculosis Complex. Antimicrobial Agents and Chemotherapy, 2004. 48(1): p. 143-150.

370. Jani Reddy Bolla, S.V.D., Feng Long, Lei Dai, Chih-Chia Su, Hsiang-Ting Lei, Xiao Chen, Jillian E. Gerkey, Daniel C. Murphy, Kanagalaghatta R. Rajashankar, Qijing Zhang, and Edward W. Yu, Structural and functional analysis of the transcriptional regulator Rv3066 of Mycobacterium tuberculosis. Nucleic Acids Research, 212. 40(18): p. 9340-9355.

371. Piddock, L.J.V., K.J. Williams, and V. Ricci, Accumulation of rifampicin by Mycobacterium aurum, Mycobacterium smegmatis and Mycobacterium tuberculosis. Journal of Antimicrobial Chemotherapy, 2000. 45(2): p. 159-165.

372. Louw, G.E., et al., Rifampicin Reduces Susceptibility to Ofloxacin in Rifampicinresistant Mycobacterium tuberculosis through Efflux. American Journal of Respiratory and Critical Care Medicine, 2011. 184(2): p. 269-276.

373. Zhang Y, S.A., Nikaido, Sun, Z., Role of acid $\mathrm{pH}$ and deficient efflux of pyrazinoic acid in unique susceptibility of Mycobacterium tuberculosis to pyrazinamide. Journal of Bacteriology, 1999. 181(7): p. 2044-2049.

374. Sun, Z. and Y. Zhang, Reduced pyrazinamidase activity and the natural resistance of Mycobacterium kansasii to the antituberculosis drug pyrazinamide. Antimicrobial Agents and Chemotherapy, 1999. 43(3): p. 537-542.

375. Machado, D., et al., Contribution of Efflux to the Emergence of Isoniazid and Multidrug Resistance in Mycobacterium tuberculosis. PLoS ONE, 2012. 7(4): p. e34538.

376. Santiago Ramón-García, V.M., Elisa Dainese, Carlos Martín, Charles J. Thompson, Edda De Rossi, Riccardo Manganelli, and José A. Aínsa, Functional and genetic characterization of the tap efflux pump in Mycobacterium bovis BCG. Antimicrobial Agents and Chemotherapy, 2012. 56(4): p. 2074-2083. 
377. Martins, M., et al., SILA 421, an inhibitor of efflux pumps of cancer cells, enhances the killing of intracellular extensively drug-resistant tuberculosis (XDR-TB). International Journal of Antimicrobial Agents, 2009. 33(5): p. 479-482.

378. Ramón-García, S., et al., Role of the Mycobacterium tuberculosis P55 Efflux Pump in Intrinsic Drug Resistance, Oxidative Stress Responses, and Growth. Antimicrobial Agents and Chemotherapy, 2009. 53(9): p. 3675-3682.

379. Monaghan, R.L. and J.F. Barrett, Antibacterial drug discovery-Then, now and the genomics future. Biochemical Pharmacology, 2006. 71(7): p. 901-909.

380. Trias J, J.V., Benz R, Porins in the cell wall of mycobacteria. Science, 1992. 258(5087): p. 1479-81.

381. Nikaido, H., Multidrug Resistance in Bacteria. Annual Review of Biochemistry, 2009. 78(1): p. 119-146.

382. Ratledge, C., The physiology of the mycobacteria. Advances in Microbial Physiology 1976. 13: p. 115-244.

383. Michael U. Shiloh, P.A., DiGiuseppe Champion, To catch a killer. What can mycobacterial models teach us about Mycobacterium tuberculosis pathogenesis? Curr Opin Microbiol. , 2010. 13(1): p. p. 86-92. .

384. Brown-Elliott, B.A. and R.J. Wallace, Clinical and Taxonomic Status of Pathogenic Nonpigmented or Late-Pigmenting Rapidly Growing Mycobacteria. Clinical Microbiology Reviews, 2002. 15(4): p. 716-746.

385. Garbe, T., et al., Expression of the Mycobacterium tuberculosis 19-kilodalton antigen in Mycobacterium smegmatis: immunological analysis and evidence of glycosylation. Infection and Immunity, 1993. 61(1): p. 260-267.

386. A H Kolk, G.T.N., O de Leeuw, S Kuijper, J D van Embden, Mycobacterium smegmatis strain for detection of Mycobacterium tuberculosis by PCR used as internal control for inhibition of amplification and for quantification of bacteria. $\mathbf{J}$ Clin Microbiol. , 1994. 32(5): p. 1354-1356.

387. Yuqing Li, J.Z., Hua Zhang, Zheng-Guo He, The characterization of conserved binding motifs and potential target genes for M. tuberculosis MtrAB reveals a link between the two-component system and the drug resistance of M. smegmatis. BMC Microbiol., 2010. 10(242). 
388. Sweeney, K.A., et al., A recombinant Mycobacterium smegmatis induces potent bactericidal immunity against Mycobacterium tuberculosis. Nat Med, 2011. 17(10): p. 1261-1268.

389. Kolk, A.H., et al., Mycobacterium smegmatis strain for detection of Mycobacterium tuberculosis by PCR used as internal control for inhibition of amplification and for quantification of bacteria. Journal of Clinical Microbiology, 1994. 32(5): p. 13541356.

390. Patru, M.-M. and M.S. Pavelka, A Role for the Class A Penicillin-Binding Protein PonA2 in the Survival of Mycobacterium smegmatis under Conditions of Nonreplication. Journal of Bacteriology, 2010. 192(12): p. 3043-3054.

391. Reyrat, J.-M. and D. Kahn, Mycobacterium smegmatis: an absurd model for tuberculosis? Trends in Microbiology, 2001. 9(10): p. 472-473.

392. Clifton E, B., III, Response to: Mycobacterium smegmatis: an absurd model for tuberculosis? Trends in Microbiology, 2001. 9(10): p. 473-474.

393. Tyagi, J.S. and D. Sharma, Mycobacterium smegmatis and tuberculosis. Trends in Microbiology, 2002. 10(2): p. 68-69.

394. Michel Denis, A.F., Micheline Pelletier, Francine Gervais, Emil Skamene, Killing of Mycobacterium smegmatis by Macrophages from Genetically Susceptible and Resistant Mice. Journal of Leukocyte Biology 1990. 47: p. p. 25-30.

395. Jordao, L., et al., On the killing of mycobacteria by macrophages. Cellular Microbiology, 2008. 10(2): p. 529-548.

396. Anes, E., et al., Dynamic life and death interactions between Mycobacterium smegmatis and J774 macrophages. Cellular Microbiology, 2006. 8(6): p. 939-960.

397. Bange, F.C., F.M. Collins, and W.R. Jacobs, Survival of mice infected with Mycobacterium smegmatis containing large DNA fragments from Mycobacterium tuberculosis. Tubercle and Lung Disease, 1999. 79(3): p. 171-180.

398. Denis A. Mitchison, A.R.M.C., Predictive in vitro Models of the Sterilizing Activity of Anti-Tuberculosis Drugs. Current Pharmaceutical Design., 2004. 10(26): p. p32853295.

399. Sala, C., et al., Simple Model for Testing Drugs against Nonreplicating Mycobacterium tuberculosis. Antimicrobial Agents and Chemotherapy, 2010. 54(10): p. $4150-4158$. 
400. Fang, F.C., Antimicrobial reactive oxygen and nitrogen species: concepts and controversies. Nat Rev Micro, 2004. 2(10): p. 820-832.

401. De Groote, M.A. and F.C. Fang, NO Inhibitions: Antimicrobial Properties of Nitric Oxide. Clinical Infectious Diseases, 1995. 21(Supplement 2): p. S162-S165.

402. Fang, F.C., Perspectives Series: Host/Pathogen Interactions. Mechanisms of Nitric Oxide-related Antimicrobial Activity. The Journal of Clinical Investigation., 1997. 99(12): p. 2818-2825.

403. Renau, T.E., et al., Structure-Activity Relationships of the Quinolone Antibacterials against Mycobacteria: Effect of Structural Changes at N-1 and C-7\%. Journal of Medicinal Chemistry, 1996. 39(3): p. 729-735.

404. Renau T. E, J.W.G., J A Dever, G E Roland, E T Joannides, M A Shapiro, J P Sanchez, S J Gracheck, J M Domagala, M R Jacobs, R C Reynolds, Structure-activity relationships of quinolone agents against mycobacteria: effect of structural modifications at the 8 position. Antimicrobial Agents and Chemotherapy., 1996. 40(10): p. 2363-2368.

405. Pennekamp, A., et al., Mycobacterium smegmatis Infection in a Healthy Woman Following a Facelift: Case Report and Review of the Literature. Annals of Plastic Surgery, 1997. 39(1): p. 80-83.

406. Newton, J.A., et al., Soft-Tissue Infection Due to Mycobacterium smegmatis: Report of Two Cases. Clinical Infectious Diseases, 1993. 16(4): p. 531-533.

407. Ergan, B., et al., Mycobacterium smegmatis pneumonia. Respirology, 2004. 9(2): p. 283-285.

408. Vonmoos S, L.P., Beer V, de Haller R., Pleuropulmonary infection caused by Mycobacterium smegmatis. Case description and literature review. Schweiz Med Wochenschr., 1986. 116(52): p. p. 1852-6.

409. Bagchi, G., Mayuri, and J.S. Tyagi, Hypoxia-responsive expression of Mycobacterium tuberculosis $R v 3134 c$ and devR promoters in Mycobacterium smegmatis. Microbiology, 2003. 149(9): p. 2303-2305.

410. O'Toole, R., et al., A Two-Component Regulator of Universal Stress Protein Expression and Adaptation to Oxygen Starvation in Mycobacterium smegmatis. Journal of Bacteriology, 2003. 185(5): p. 1543-1554.

411. Mayuri, et al., Molecular analysis of the dormancy response in Mycobacterium smegmatis: expression analysis of genes encoding the DevR-DevS two-component 
system, Rv3134c and chaperone $\alpha$-crystallin homologues. FEMS Microbiology Letters, 2002. 211(2): p. 231-237.

412. Edith NG Houben, A.W., Giorgio Ferrari, Liem Nguyen, Charles J Thompson, Christian Miess, Guido Vogel, Bernd Mueller, Jean Pieters, Differential expression of a virulence factor in pathogenic and non-pathogenic mycobacteria. Mol Microbiol. , 2009. 72(1): p. 41-52.

413. Aguinaldo, A.M., et al., Quinoline alkaloids from Lunasia amara inhibit Mycobacterium tuberculosis H37Rv in vitro. International Journal of Antimicrobial Agents, 2007. 29(6): p. 744-746.

414. Stein Hans, H.P., Enzyme from calf thymus degrading the RNA moiety of DNA-RNA Hybrids: effect on DNA-dependent RNA polymerase. Science, 1969. 166(3903): p. 393-395.

415. Dasgupta, S., H. Masukata, and J.-i. Tomizawa, Multiple mechanisms for initiation of ColE1 DNA replication: DNA synthesis in the presence and absence of ribonuclease H. Cell, 1987. 51(6): p. 1113-1122.

416. Ogawa, T., et al., RNase $H$ confers specificity in the dnaA-dependent initiation of replication at the unique origin of the Escherichia coli chromosome in vivo and in vitro. Proceedings of the National Academy of Sciences, 1984. 81(4): p. 1040-1044.

417. Horiuchi T, M.H., Sekiguchi M., RNase H-defective mutants of Escherichia coli: a possible discriminatory role of RNase $H$ in initiation of DNA replication. Mol Gen Genet. , 1984. 195(1-2): p. 17-22.

418. Kogoma T, S.N., von Meyenburg K, Function of ribonuclease $H$ in initiation of DNA replication in Escherichia coli K-12. Mol Gen Genet. , 1985. 200(1): p. 103-9.

419. Cerritelli, S.M. and R.J. Crouch, Ribonuclease H: the enzymes in eukaryotes. FEBS Journal, 2009. 276(6): p. 1494-1505.

420. Watkins, H.A. and E.N. Baker, Structural and Functional Characterization of an RNase HI Domain from the Bifunctional Protein Rv2228c from Mycobacterium tuberculosis. J. Bacteriol., 2010. 192(11): p. 2878-2886.

421. Andries, K., et al., A Diarylquinoline Drug Active on the ATP Synthase of Mycobacterium tuberculosis. Science, 2005. 307(5707): p. 223-227.

422. Selik, R.M., Haverkos, H. W., Curran, J. W., Acquired immune deficiency syndrome (AIDS) trends in the United States, 1978-1982. The American Journal of Medicine, 1984. 76(3): p. 493-500. 
423. Gallo RC, S.P., Gelmann EP, Robert-Guroff M, Richardson E, Kalyanaraman VS, Mann D, Sidhu GD, Stahl RE, Zolla-Pazner S, Leibowitch J, Popovic M, Isolation of human T-cell leukemia virus in acquired immune deficiency syndrome (AIDS). Science, 1983. 220(4599): p. 865-7.

424. Barré-Sinoussi F, C.J., Rey F, Nugeyre MT, Chamaret S, Gruest J, Dauguet C, AxlerBlin C, Vézinet-Brun F, Rouzioux C, Rozenbaum W, Montagnier L, Isolation of a Tlymphotropic retrovirus from a patient at risk for acquired immune deficiency syndrome (AIDS). Science, 1983. 220(4599): p. 868-71.

425. Perri, G.D., et al., Transmission of HIV-Associated Tuberculosis to Healthcare Workers. Infection Control and Hospital Epidemiology, 1993. 14(2): p. 67-72.

426. Richards SB, S.L.M., Nieburg P, Coulibaly IM, Coulibaly D, Abouya L, Gayle HD, De Cock KM., Impact of the HIV epidemic on trends in tuberculosis in Abidjan, Côte d'Ivoire. Tuber Lung Dis., 1995. 76(1): p. 11-6.

427. Glynn, J.R., Resurgence of tuberculosis and the impact of HIV infection. British Medical Bulletin, 1998. 54(3): p. 579-593.

428. Catherine F. Decker, A.L., Tuberculosis and HIV infection. How to safely treat both disorders concurrently. Postgrad Med., 2000. 108(2): p. 57-68.

429. S Bhagyabati Devi, S.N., T Jeetenkumar Singh, Ksh Birendra Singh, Lallan Prasad, Th Shanti Devi, HIV and TB Co-infection: A Study from RIMS Hospital, Manipur. Journal, Indian Academy of Clinical Medicine, 2005. 6(3): p. 220-223.

430. Steinbrook, R., Tuberculosis and HIV in India. New England Journal of Medicine, 2007. 356(12): p. 1198-1199.

431. Gupta, S., et al., Role of risk factors and socio-economic status in pulmonary tuberculosis: a search for the root cause in patients in a tertiary care hospital, South India. Tropical Medicine \& International Health, 2011. 16(1): p. 74-78.

432. Selwyn, P.A., et al., A Prospective Study of the Risk of Tuberculosis among Intravenous Drug Users with Human Immunodeficiency Virus Infection. New England Journal of Medicine, 1989. 320(9): p. 545-550.

433. Schluger, N.W., HIV-Associated Tuberculosis: Clinical Challenges. In AIDS and Tuberculosis: A Deadly Liaison, (Ed.) Stefan H. E. Kaufmann, Bruce D. Walker. WILEY-VCH Verlag GmbH \& Co. KGaA, 2009.

434. Del Amo, J., et al., Does tuberculosis accelerate the progression of HIV disease? Evidence from basic science and epidemiology. AIDS, 1999. 13(10): p. 1151-1158. 
435. Whalen, C., et al., Accelerated course of human immunodeficiency virus infection after tuberculosis. Am. J. Respir. Crit. Care Med., 1995. 151(1): p. 129-135.

436. Boris Julg, B.D.W., HIV Immunology and Prospects for Vaccines. In AIDS and Tuberculosis: A Deadly Liaison, (Ed.) Stefan H. E. Kaufmann and Bruce D. Walker. 2009.

437. Arvind Venkat, D.M.P., Robert R. Cooney, Adarsh K. Srivastava, Gregory A. Suares, Cory P. Heidelberger., Care of the HIV-Positive Patient in the Emergency Department in the Era of Highly Active Antiretroviral Therapy. Infectious Disease: Annals of Emergency Medicine, 2008. 52(3): p. 274-285.

438. Lima VD, H.R., Harrigan PR, Moore D, Yip B, Wood E, Montaner JS, Continued improvement in survival among HIV-infected individuals with newer forms of highly active antiretroviral therapy. AIDS., 2007. 21(6): p. 685-92.

439. Palella, F.J., et al., Declining Morbidity and Mortality among Patients with Advanced Human Immunodeficiency Virus Infection. New England Journal of Medicine, 1998. 338(13): p. 853-860.

440. Powderly, W.G., A. Landay, and M.M. Lederman, Recovery of the Immune System with Antiretroviral Therapy. The Journal of the American Medical Association, 1998. 280(1): p. 72-77.

441. World Health Organization. United Nations Programme on HIV/AIDS. Report on the global HIV/AIDS epidemic (1998). Available at http://data.unaids.org/pub/Report/1998/19981125_global_epidemic_report_en.pdf. Retrieved on December 2011.

442. Sepkowitz, K.A., AIDS - The First 20 Years. New England Journal of Medicine, 2001. 344(23): p. 1764-1772.

443. World Health Organisation, AIDS epidemic update. UNAIDS report on the global AIDS $\quad 2006 . \quad$ Available at http://data.unaids.org/pub/epireport/2006/2006_epiupdate_en.pdf.

444. World Health Organization. Joint United Nations Programme on HIV/AIDS. UNAIDS Data Tables $2011 . \quad$ Available at http://www.unaids.org/en/media/unaids/contentassets/documents/unaidspublication/2 011/JC2225 UNAIDS datatables en.pdf. Accessed on December 2011. 
445. WHO-UNAIDS Global report: UNAIDS report on the global AIDS epidemic 2010. Available http://www.unaids.org/globalreport/documents/20101123 GlobalReport full en.pdf

446. Cantwell, M.F. and N.J. Binkin, Tuberculosis in sub-Saharan Africa: A regional assessment of the impact of the human immunodeficiency virus and National Tuberculosis Control Program quality. Tubercle and Lung Disease, 1996. 77(3): p. 220-225.

447. Global Alliance for TB Drug Development. 2011 Annual Report: Crossing Thresholds. Available at www.tballiance.org. Accessed on December 2011.

448. Leroy, V., et al., Progression of Human Immunodeficiency Virus Infection in Patients with Tuberculosis Disease. American Journal of Epidemiology, 1997. 145(4): p. 293300.

449. Helen McIlleron, G.M., William J. Burman, and Gary Maartens, Complications of Antiretroviral Therapy in Patients with Tuberculosis: Drug Interactions, Toxicity, and Immune Reconstitution Inflammatory Syndrome. The Journal of Infectious Diseases, 2007. 196((Suppl 1)): p. S63-75.

450. Fountain, F.F., et al., Isoniazid Hepatotoxicity Associated With Treatment of Latent Tuberculosis Infection: A 7-Year Evaluation From a Public Health Tuberculosis Clinic. Chest, 2005. 128(1): p. 116-123.

451. Fekih, L., Boussoffara, L., Fenniche, S., Abdelghaffar, H., Megdiche, M. L., Neuropsychiatric side effects of antituberculosis agents. Revue Medicale Liege, 2011. 66(2): p. 82-85.

452. Montessori, V., et al., Adverse effects of antiretroviral therapy for HIV infection. Canadian Medical Association Journal, 2004. 170(2): p. 229-238.

453. Carr, A. and D.A. Cooper, Adverse effects of antiretroviral therapy. The Lancet, 2000. 356(9239): p. 1423-1430.

454. Tseng, S.-H., et al., Impact of HAART Therapy on Co-Infection of Tuberculosis and HIV Cases for 9 Years in Taiwan. The American Journal of Tropical Medicine and Hygiene, 2009. 80(4): p. 675-677.

455. Manosuthi W, C.S., Thongyen S, Chaovavanich A, Sungkanuparph S., Survival rate and risk factors of mortality among HIV/tuberculosis-coinfected patients with and without antiretroviral therapy. Journal of acquired immune deficiency syndromes., 2006. 43(1): p. 42-46. 
456. Nowotny, M., et al., Crystal Structures of RNase H Bound to an RNA/DNA Hybrid: Substrate Specificity and Metal-Dependent Catalysis. Cell, 2005. 121(7): p. 10051016.

457. Klarmann GJ, H.M., Le Grice SF., Uncovering the complexities of retroviral ribonuclease $H$ reveals its potential as a therapeutic target. AIDS Review, 2002. 4(4): p. 183-94.

458. Tisdale, M., et al., Mutations within the RNase $H$ Domain of Human Immunodeficiency Virus Type 1 Reverse Transcriptase Abolish Virus Infectivity. Journal of General Virology, 1991. 72(1): p. 59-66.

459. F. Esposito, L.Z., E. Tramontano, Antiviral drug discovery: HIV-1 RNase H, the next hit target. Communicating Current Research and Educational Topics and Trends in Applied Microbiology. , 2007: p. p. 799-809.

460. Gong, Q., et al., Interaction of HIV-1 Reverse Transcriptase Ribonuclease $H$ with an Acylhydrazone Inhibitor. Chemical Biology \& Drug Design, 2011. 77(1): p. 39-47.

461. Grandi, M.D., et al., Small molecule inhibitors of HIV RT Ribonuclease H. Bioorganic \&amp; Medicinal Chemistry Letters, 2010. 20(1): p. 398-402.

462. Su, H.-P., et al., Structural Basis for the Inhibition of RNase H Activity of HIV-1 Reverse Transcriptase by RNase $H$ Active Site-Directed Inhibitors. Journal of Virology, 2010. 84(15): p. 7625-7633.

463. Andreola, M.-L., et al., DNA Aptamers Selected against the HIV-1 RNase H Display in Vitro Antiviral Activity. Biochemistry, 2001. 40(34): p. 10087-94.

464. Cowley, S., The biology of HIV infection. Leprosy review, 2001. 72(2): p. 212-220.

465. Hu, Y.W. and C.Y. Kang, Enzyme activities in four different forms of human immunodeficiency virus 1 pol gene products. Proceedings of the National Academy of Sciences, 1991. 88(11): p. 4596-4600.

466. Fang Yu, X.L., Peng Zhan, Erik De Clercq Recent Advances in the Research of HIV-1 RNase H Inhibitors Mini Rev Med Chem. , 2008. 8(12): p. 1243-1251.

467. Greg L. Beilhartz, M.G., HIV-1 Ribonuclease H: Structure, Catalytic Mechanism and Inhibitors. Viruses., 2010. 2(4): p. 900-926.

468. Dirk, J., Novel HIV-1 reverse transcriptase inhibitors. Virus Research, 2008. 134(1-2): p. 171-185.

469. Klaus Klumpp, T.M., Recent Progress in the Design of Small Molecule Inhibitors of HIV RNase H. Current Pharmaceutical Design, 2006. 12(15): p. 1909-1922. 
470. Wendeler, M., et al., Vinylogous Ureas as a Novel Class of Inhibitors of Reverse Transcriptase-Associated Ribonuclease H Activity. ACS Chemical Biology, 2008. 3(10): p. 635-644.

471. Akey, D.L. and J.M. Berger, Structure of the nuclease domain of ribonuclease III from M. tuberculosis at 2.1 A. Protein Science, 2005. 14(10): p. 2744-2750.

472. Parniak, M.A., et al., A fluorescence-based high-throughput screening assay for inhibitors of human immunodeficiency virus-1 reverse transcriptase-associated ribonuclease H activity. Analytical Biochemistry, 2003. 322(1): p. 33-39.

473. Corbett, E.L., et al., The Growing Burden of Tuberculosis: Global Trends and Interactions With the HIV Epidemic. Arch Intern Med, 2003. 163(9): p. 1009-1021.

474. Nsanzabana, C. and P.J. Rosenthal, In Vitro Activity of Antiretroviral Drugs against Plasmodium falciparum. Antimicrobial Agents and Chemotherapy, 2011. 55(11): p. 5073-5077.

475. Stefano Donadio, L.C., Letizia Brandi, Stefania Serina, Adolfo Soffientini, Elena Raimondi, Nicoletta Montanini, Margherita Sosio, Claudio O Gualerzi Targets and assays for discovering novel antibacterial agents. Journal of Biotechnology 2002. 99(3): p. 175-185.

476. Robert A, G., Jr., Hit and lead identification: Integrated technology-based approaches. Drug Discovery Today: Technologies, 2006. 3(4): p. 367-375.

477. Turner, D.J., et al., An ex vivo Culture Model for Screening Drug Activity against in vivo Phenotypes of Mycobacterium tuberculosis. Microbiology, 2002. 148(10): p. 2929-2936.

478. Christophe Marchand, J.A.B., Antony Wamiru, Scott Budihas, Ute Mo“llmann, Lothar Heinisch, John W. Mellors, Stuart F. Le Grice, Yves Pommier, Madurahydroxylactone Derivatives as Dual Inhibitors of Human Immunodeficiency Virus Type 1 Integrase and RNase H. Antimicrobial Agents and Chemotherapy, 2008. 52(1): p. 361-364.

479. Betts, J.C., et al., Evaluation of a nutrient starvation model of Mycobacterium tuberculosis persistence by gene and protein expression profiling. Molecular Microbiology, 2002. 43(3): p. 717-731.

480. Fisher, M.A., B.B. Plikaytis, and T.M. Shinnick, Microarray Analysis of the Mycobacterium tuberculosis Transcriptional Response to the Acidic Conditions Found in Phagosomes. Journal of Bacteriology, 2002. 184(14): p. 4025-4032. 
481. Hussain, S., et al., In Vitro Model of Mycobacterial Growth Arrest Using Nitric Oxide with Limited Air. Antimicrobial Agents and Chemotherapy, 2009. 53(1): p. 157-161.

482. Haruaki Tomioka, K.S., Chiaki Sano, Keisuke Sano, Toshiaki Shimizu, Intramacrophage Passage of Mycobacterium tuberculosis and M. avium Complex Alters the Drug Susceptibilities of the Organisms as Determined by Intracellular Susceptibility Testing Using Macrophages and Type II Alveolar Epithelial Cells. Antimicrob Agents Chemother., 2002. 46(2): p. 519-521.

483. Besra, G.S., Chatterjee D, Lipids and carbohydrates of Mycobacterium tuberculosis. In Tuberculosis; (Ed.) Bloom B. R. ASM Press, Washington DC, 1994: p. p. 285-306.

484. Protopopova, M., et al., Identification of a new antitubercular drug candidate, SQ109, from a combinatorial library of 1,2-ethylenediamines. Journal of Antimicrobial Chemotherapy, 2005. 56(5): p. 968-974.

485. Lee Jia, J.E.T., Colleen Hanrahan,Lori Coward, Patricia Noker, Gregory Gorman, Boris Nikonenko, Marina Protopopova, Pharmacodynamics and pharmacokinetics of SQ109, a new diamine-based antitubercular drug. British Journal of Pharmacology, 2005. 144(1): p. 80-87.

486. Richard E. Lee, M.P., Emma Crooks, Richard A. Slayden, Marianne Terrot, Clifton E. Barry III, Combinatorial Lead Optimization of [1,2]-Diamines Based on Ethambutol as Potential Antituberculosis Preclinical Candidates. Journal of Combinatorial Chemistry, 2003. 5(2): p. 172-187.

487. Shaw-Reid, C.A., et al., Inhibition of HIV-1 Ribonuclease H by a Novel Diketo Acid, 4-[5-(Benzoylamino)thien-2-yl]-2,4-dioxobutanoic Acid. Journal of Biological Chemistry, 2003. 278(5): p. 2777-2780.

488. Kirschberg, T.A., et al., RNase H Active Site Inhibitors of Human Immunodeficiency Virus Type 1 Reverse Transcriptase: Design, Biochemical Activity, and Structural Information. Journal of Medicinal Chemistry, 2009. 52(19): p. 5781-5784.

489. Budihas, S.R., et al., Selective inhibition of HIV-1 reverse transcriptase-associated ribonuclease $H$ activity by hydroxylated tropolones. Nucleic Acids Research, 2005. 33(4): p. 1249-1256.

490. S Loya, R.T., Y Kashman, A Hizi, Illimaquinone, a selective inhibitor of the RNase H activity of human immunodeficiency virus type 1 reverse transcriptase. Antimicrobial Agents and Chemotherapy, 1990. 34(10): p. 2009-2012. 
491. Borkow, G., et al., Inhibition of the Ribonuclease H and DNA Polymerase Activities of HIV-1 Reverse Transcriptase by N-(4-tert-Butylbenzoyl)-2-hydroxy-1naphthaldehyde Hydrazone. Biochemistry, 1997. 36(11): p. 3179-3185.

492. Tributino, J.L.M., et al., Novel 6-methanesulfonamide-3,4-methylenedioxyphenyl-Nacylhydrazones: Orally effective anti-inflammatory drug candidates. Bioorganic \&amp; Medicinal Chemistry, 2009. 17(3): p. 1125-1131.

493. Tan, Z., et al., Screening and evaluation of thiourea derivatives for their HIV capsid and human cyclophilin A inhibitory activity. Medicinal Chemistry Research, 2011. 20(3): p. 314-320.

494. Jin, Y., et al., SAR and molecular mechanism study of novel acylhydrazone compounds targeting HIV-1 CA. Bioorganic \&amp; Medicinal Chemistry, 2010. 18(6): p. 2135-2140.

495. Daniel M. Himmel, S.G.S., Sanjeewa Dharmasena, Mohammed M. Hossain, Kessler McCoy-Simandle, Tatiana Ilina, Arthur D. Clark, Jr., Jennifer L. Knight, John G. Julias, Patrick K. Clark, Karsten Krogh-Jespersen, Ronald M. Levy, Stephen H. Hughes, Michael A. Parniak, Eddy Arnold, HIV-1 Reverse Transcriptase Structure with RNase H Inhibitor Dihydroxy Benzoyl Naphthyl Hydrazone Bound at a Novel Site. ACS Chem Biol., 2006. 1(11): p. 702-712.

496. Gasparro, D.M., et al., Reaction profiling of the MAO-B catalyzed oxidative deamination of amines in Alzheimer's disease. Journal of Molecular Structure: THEOCHEM, 2003. 666-667(0): p. 527-536.

497. Chemical Structures of HIV RNase $H$ Inhibitors. Availabe at http://www.ncbi.nlm.nih.gov/pccompound. Retrieved on December 2011.

498. Xu, Z.-Q., et al., Anti-HIV natural product (+)-calanolide A is active against both drug-susceptible and drug-resistant strains of Mycobacterium tuberculosis. Bioorganic \&amp; Medicinal Chemistry, 2004. 12(5): p. 1199-1207.

499. Elena A. Semenova, A.A.J., Christophe Marchand, David A. Davis, Robert Yarchoan, Yves Pommier, Preferential Inhibition of the Magnesium-Dependent Strand Transfer Reaction of HIV-1 Integrase by $\alpha$-Hydroxytropolones. Molecular Pharmacology, 2006. 69(4): p. 1454-1460.

500. Koul, A., et al., The challenge of new drug discovery for tuberculosis. Nature, 2011. 469(7331): p. 483-490. 
501. Global Alliance for TB Drug Development. TB Alliance Annual Report 2010. Available at www.tballiance.org. .

502. Ji, B., et al., In Vitro and In Vivo Activities of Moxifloxacin and Clinafloxacin against Mycobacterium tuberculosis. Antimicrobial Agents and Chemotherapy, 1998. 42(8): p. 2066-2069.

503. Palomino, J.C., D.F. Ramos, and P.A. da Silva, New Anti-Tuberculosis Drugs: Strategies, Sources and New Molecules. Current Medicinal Chemistry, 2009. 16(15): p. $1898-1904$.

504. Candice K. Kwan, J.D.E., HIV and Tuberculosis: a Deadly Human Syndemic. Clinical Microbiology Reviews 2011. 24(2): p. p. 351-376.

505. Altaf, M., et al., Evaluation of the Mycobacterium smegmatis and BCG models for the discovery of Mycobacterium tuberculosis inhibitors. Tuberculosis, 2010. 90(6): p. 333-337.

506. David L. Hahn, A.A.A., Wandy L. Beatty and Gerald I. Byrne, Chlamydia Pneumoniae As A Respiratory Pathogen. Frontiers in Bioscience 2002. 7: p. e66-76.

507. Florescu DF, K.A., Cytomegalovirus Infections in Non-immunocompromised and Immunocompromised Patients in the Intensive Care Unit. Infect Disord Drug Targets., 2011(Epub ahead of print).

508. Corti, M., et al., Human herpesvirus 6: report of emerging pathogen in five patients with HIV/AIDS and review of the literature. Revista da Sociedade Brasileira de Medicina Tropical, 2011. 44: p. 522-525.

509. Morens, D.M., G.K. Folkers, and A.S. Fauci, The challenge of emerging and reemerging infectious diseases. Nature, 2004. 430(6996): p. 242-249.

510. Kwan, C.K. and J.D. Ernst, HIV and Tuberculosis: a Deadly Human Syndemic. Clin. Microbiol. Rev., 2011. 24(2): p. 351-376.

511. White, N.J., Drug resistance in malaria. British Medical Bulletin - The British Council, 1998. 54(3): p. 703-715.

512. Nicola, G., et al., Discovery of novel inhibitors targeting enoyl-acyl carrier protein reductase in Plasmodium falciparum by structure-based virtual screening. Biochemical and Biophysical Research Communications, 2007. 358(3): p. 686-691.

513. Ron Peek, T.V.G., Daynand Panchoe, Sophie Greve, Ellen Bus, Lesley Resida, Drug Resistance and Genetic Diversity of Plasmodium falciparum Parasties from Suriname. The American Journal of Tropical Medicine and Hygiene, 2005. 73(5): p. 833-838. 
514. Dondorp, A.M., et al., Artemisinin Resistance in Plasmodium falciparum Malaria. New England Journal of Medicine, 2009. 361(5): p. 455-467.

515. Chansuda Wongsrichanalai, S.R.M., Declining Artesunate-Mefloquine Efficacy against Falciparum Malaria on the Cambodia-Thailand Border. Emerging Infectious Diseases, 2008. 14(5): p. 716-719.

516. Tadokoro, T. and S. Kanaya, Ribonuclease H: molecular diversities, substrate binding domains, and catalytic mechanism of the prokaryotic enzymes. FEBS Journal, 2009. 276(6): p. 1482-1493.

517. L W Scheibel, A.A., W Trager, Tetraethylthiuram disulfide (Antabuse) inhibits the human malaria parasite Plasmodium falciparum. Proc Natl Acad Sci USA., 1979. 76(10): p. 5303-5307. .

518. Murikoli, R.V., Discovery of novel anti-tuberculosis compounds using an itramacrophage assay. MS thesis. School of Biological Sciences, Victoria University of Wellington, New Zealand. 2010.

519. Dobos, K.M., et al., Necrosis of Lung Epithelial Cells during Infection with Mycobacterium tuberculosis is Preceded by Cell Permeation. Infection and Immunity, 2000. 68(11): p. 6300-6310.

520. Fratazzi, C., et al., Macrophage apoptosis in mycobacterial infections. Journal of Leukocyte Biology, 1999. 66(5): p. 763-764.

521. De Groote, M.A., et al., Comparative Studies Evaluating Mouse Models Used for Efficacy Testing of Experimental Drugs against Mycobacterium tuberculosis. Antimicrobial Agents and Chemotherapy, 2011. 55(3): p. 1237-1247.

522. Mycobacterium tuberculosis H37Rv. TubercuList, Version 2.4. Available at http://tuberculist.epfl.ch/index.html accessed on November 2011.

523. Hall, L., et al., Use of Hydrogen Peroxide Vapor for Deactivation of Mycobacterium tuberculosis in a Biological Safety Cabinet and a Room. J. Clin. Microbiol., 2007. 45(3): p. 810-815.

524. pET28a (+) Vectors: Novagen. Plasmid map available at http://www.genomex.com/vector_maps/pET28_map.pdf, retrieved January 2012.

525. Takayama, K., C. Wang, and G.S. Besra, Pathway to Synthesis and Processing of Mycolic Acids in Mycobacterium tuberculosis. Clinical Microbiology Reviews, 2005. 18(1): p. 81-101. 
526. Riley, L.W., Of mice, men, and elephants: Mycobacterium tuberculosis cell envelope lipids and pathogenesis. The Journal of Clinical Investigation, 2006. 116(6): p. 14751478. 
Appendices 


\subsection{Miscellaneous Tables:}

Table 7.1: Recommended chemotherapy for active pulmonary $\mathrm{Tb}$

\begin{tabular}{lcc}
\hline Standard Drug & \multicolumn{2}{c}{${ }^{\mathrm{a}}$ Recommended Regimen for Tb } \\
\cline { 2 - 3 } & ${ }^{\mathrm{b}}$ First Two Months & ${ }^{\mathrm{c}}$ Next Four Months \\
\hline Rifampicin & + & + \\
Isoniazid & + & + \\
Pyrazinamide & + & - \\
Ethambutol & & - \\
\hline
\end{tabular}

${ }^{a}$ World Health Organization (Treatment of Tuberculosis). ${ }^{b}$ Initial intensive phase with combination therapy. ${ }^{c}$ Continuation phase for next at least four months depending on stage of the disease. $(+)$ indicates required, (-) indicates, not required.

Table 7.2: PCR steps used to amplify M. tuberculosis genes

\begin{tabular}{lccc}
\hline PCR Step & Temperature & Time & No of Cycles \\
\hline Initial Denature & $95{ }^{\circ} \mathrm{C}$ & 5 min & 1 \\
\hline Denature & $94{ }^{\circ} \mathrm{C}$ & 1 min & $25 \mathrm{x}$ \\
Anneal & $55^{\circ} \mathrm{C}$ & $30 \mathrm{sec}$ & 1 \\
Extension & $72{ }^{\circ} \mathrm{C}$ & $1 \mathrm{~min}$ & \\
\hline Final Extension & $72{ }^{\circ} \mathrm{C}$ & $5 \mathrm{~min}$ & \\
& $4{ }^{\circ} \mathrm{C}$ & & \\
Hold & & & \\
\hline
\end{tabular}

DNA quantity was confirmed by NanoDrop® ND-100 Spectrophotometer (NanoDrop Technologies, New Zealand) using the software 3.1.0. ( $\infty$ ) indicates infinity. 
Table 7.3: Concentrations for restriction digestion

\begin{tabular}{lll} 
Reagent & Stock Conc. & Final Conc. \\
\hline Enzyme & $10,000 \mathrm{U} / \mu \mathrm{L}$ & $0.5 \mathrm{U} / \mu \mathrm{L}$ \\
NEB buffer & $10 \mathrm{x}$ & $1 \mathrm{x}$ \\
BSA & $10 \mathrm{x}$ & $1 \mathrm{x}$ \\
Vector & $\sim 1 \mu \mathrm{g}$ & $25-50 \mathrm{ng} / \mu \mathrm{L}$ \\
\hline
\end{tabular}

Table 7.4: Reaction set up for double restriction digestion

\begin{tabular}{lcc}
\hline Reagents & PCR Product & Vector \\
\hline ddH2O & $5 \mu \mathrm{L}$ & $5 \mu \mathrm{L}$ \\
Buffer 4 (10x) & $2 \mu \mathrm{L}$ & $2 \mu \mathrm{L}$ \\
BSA (1x) & $1.0 \mu \mathrm{L}$ & $1.0 \mu \mathrm{L}$ \\
DNA & $10 \mu \mathrm{L}$ & $10 \mu \mathrm{L}$ \\
EcoRI & $1.0 \mu \mathrm{L}$ & $1.0 \mu \mathrm{L}$ \\
XhoI & $1.0 \mu \mathrm{L}$ & $1.0 \mu \mathrm{L}$ \\
\hline
\end{tabular}

A total of $20 \mu \mathrm{L}$ reaction was used for restriction digestion experiments and all reagents are in order of addition.

Different vector-to-insert molar ratios were tested in order to evaluate the appropriate transformation efficiency. Positive control (uncut pET28a (+) into DH5 $\alpha \mathrm{e}^{-}$) and negative control (water instead of plasmid) were included in each experiment. 
Table 7.5: Conditions for gel electrophoresis

\begin{tabular}{lll} 
Conditions & PCR Products & Restriction Digests \\
\hline Agarose conc. & $1 \%$ & $0.8 \%$ \\
Buffer & $1 \mathrm{x} \mathrm{TAE}$ & $1 \mathrm{x}$ TAE \\
Time & 40 minutes & 80 minutes \\
Voltage & $120-140 \mathrm{~V}$ & $80 \mathrm{~V}$ \\
& & \\
Amperes & $400 \mathrm{~A}$ & $300 \mathrm{~A}$ \\
\hline
\end{tabular}

Table 7.6: DNA concentration of PCR_Rv2228c

\begin{tabular}{llcc}
\hline Tube No. & Volume & DNA Conc. & DNA Purity ${ }^{260 / 280}$ \\
\hline 1 & $50 \mu \mathrm{L}$ & $36.3 \mathrm{ng} / \mu \mathrm{L}$ & 1.78 \\
2 & $50 \mu \mathrm{L}$ & $39.1 \mathrm{ng} / \mu \mathrm{L}$ & 1.81 \\
3 & $50 \mu \mathrm{L}$ & $37.4 \mathrm{ng} / \mu \mathrm{L}$ & 1.79 \\
\hline
\end{tabular}

As per supplier's recommendations, a maximum of $1 \mu \mathrm{L}$ of restriction enzyme was used in restriction digestion experiments because pre-added glycerol (for storage) can affect the working efficiency of restriction enzymes.

Four eppendorfs averaging $50 \mu \mathrm{L}$ of pET28a $(+)$ was recovered from DH5 $\alpha$ cells after overnight incubation at $37{ }^{\circ} \mathrm{C}$.

\section{Results for pET28a (+) Miniprep}

Plasmid was extracted using plasmid extraction kit. 


\subsubsection{DNA Concentration and Quantification}

Quantity of DNA was confirmed through NanoDrop, which was zero blank against DNA concentration at $0.0 \mathrm{ng} / \mu \mathrm{L}$ with water prior to take readings. Plasmid preparations were cleaned using a plasmid clean kit and DNA and DNA was quantified using a NanoDrop ${ }^{\mathrm{TM}}$ (NanoDrop Technologies, Auckland, New Zealand). Concentrations of plasmid DNA is given below.

Table 7.7: DNA concentration of an extracted recombinant plasmid

\begin{tabular}{llll}
\hline Tube No. & Volume & DNA Conc. & DNA Purity ${ }^{260 / 280}$ \\
\hline 1 & $50 \mu \mathrm{L}$ & $21.4 \mathrm{ng} / \mu \mathrm{L}$ & 1.89 \\
2 & $50 \mu \mathrm{L}$ & $20.6 \mathrm{ng} / \mu \mathrm{L}$ & 2.2 \\
3 & $50 \mu \mathrm{L}$ & $23.7 \mathrm{ng} / \mu \mathrm{L}$ & 1.87 \\
4 & $50 \mu \mathrm{L}$ & $22.1 \mathrm{ng} / \mu \mathrm{L}$ & 2.0 \\
\hline
\end{tabular}

Plasmid DNA was extracted using High Speed Mini ${ }^{\mathrm{TM}}$ plasmid DNA extraction kit 
Table 7.8: DNA sequence for $M$. tuberculosis genes encoding RNase HI/II

$M t b$ ORF Rv2228c

Length DNA Sequence (1095 base pairs)

GTGAAAGTTGTCATCGAAGCCGACGGCGGATCGCGGGGCAATCCCGGACCGGCCGGATACGGCGC GGTGGTGTGGACCGCCGATCACTCCACCGTGCTGGCCGAGTCCAAGCAGGCGATCGGCCGGGCGA CGAACAACGTCGCCGAATACCGCGGCCTGATAGCCGGTTTGGACGACGCCGTGAAACTAGGTGCC ACCGAGGCCGCGGTGCTGATGGACTCCAAGCTGGTGGTGGAGCAGATGTCCGGGCGGTGGAAGGT CAAGCACCCGGATCTGCTGAAGCTCTATGTCCAGGCTCAGGCGTTGGCGTCCCAGTTTCGCAGGAT CAACTACGAGTGGGTTCCGCGTGCCCGGAACACGTATGCAGACCGGTTGGCCAATGACGCGATGG ACGCCGCCGCGCAATCGGCTGCGGCGGATGCGGATCCTGCCAAAATCGTTGCGACCGAGTCACC GACATCTCCCGGCTGGACCGGCGCCCGCGGTACACCCACCCGACTACTTTTGTTGCGCCACGGGCA GACGGAGCTGTCGGAGCAACGCCGCTATTCGGGGCGCGGCAACCCGGGGTTGAACGAGGTGGGGT GGCGCCAGGTTGGTGCGGCGGCCGGGTATCTGGCGCGGCGCGGCGGGATCGCTGCGGTGGTCTCC TCGCCGCTACAGCGGGCTTACGACACCGCGGTGACCGCCGCCAGAGCCCTGGCCCTGGACGTGGT CGTCGATGACGACCTGGTCGAGACCGACTTCGGCGCCTGGGAGGGGCTGACGTTCGCGGAGGCCG CAGAACGCGATCCCGAGCTGCACCGTCGCTGGCTGCAGGACACCAGCATCACGCCCCCGGGTGGG GAAAGCTTCGACGACGTGCTGCGGCGGGTTCGGCGGGGACGTGATCGGATCATCGTTGGCTACG AAGGCGCGACGGTGCTGGTGGTGTCACATGTCACGCCGATCAAAATGTTGTTGCGGCTGGCGTTGG ATGCCGGGTCGGGCGTCCTATATCGGTTGCATCTTGATCTGGCATCGCTGAGCATCGCCGAGTTCT ACGCCGATGGGGCATCGTCGGTGCGATTGGTGAATCAG

$M t b$ ORF Rv2902c Length DNA Sequence (795 base pairs)

ATGACCAAGACCTGGCCACCGCGGACGGTGATCCGTAAATCTGGGGGTTTGCGGGGAATGCGCAC CCTGGAGTCCGCGCTGCATCGTGGTGGTCTGGGGCCGGTGGCCGGGGTCGACGAAGTGGGTCGCG GCGCCTGCGCTGGCCCGTTGGTGGTCGCGGCCTGCGTGCTTGGCCCCGGGCGGATAGCAAGTCTTG CCGCCCTTGATGATTCGAAGAAGCTCAGCGAGCAAGCGCGGGAGAAGCTGTTCCCGCTGATCTGC CGGTATGCGGTGGCCTATCACGTGGTGTTCATCCCGTCGGCCGAGGTCGACCGCCGCGGCGTGCAC GTGGCCAATATCGAAGGTATGCGGCGCGCGGTGGCCGGTCTGGCGGTGCGGCCCGGTTATGTGCT CAGCGACGGCTTTCGCGTGCCAGGACTGCCGATGCCGTCGTTGCCGGTGATCGGTGGCGACGCGG CAGCCGCCTGCATCGCCGCGGCTAGCGTGCTCGCCAAGGTCAGCCGGGACCGCGTGATGGTTGCG 
TTGGACGCCGATCACCCCGGGTACGGTTTCGCGGAGCACAAGGGTTACAGCACCCCGGCGCACAG CCGGGCGCTGGCCCGATTGGGACCCTGCCCGCAGCACCGTTACTCGTTCATCAATGTTCGCCGGGT GGCGAGTGGGTCGAACACCGCAGAGGTCGCCGACGGCCAACCCGACCCTCGGGACGGGACGGCA CAGACGGGTGAGGGAAGATGGAGCAAGAGTTCCCACCCAGCGACAATGCGGGCCACGGGTCGCG CTCAGGGCACTTAG

For Rv2228c, BamHI and HindIII restriction sites at 423 bp and at 849 bp, respectively were found in gene sequence that encodes for RNase HI in M. tuberculosis (indicated in bold) therefore these restriction sites were not used for cloning Rv2228c. Gentle Software was used to design primers required for cloning experiments and DNA sequences of Rv2228c and Rv2902c were retrieved from [522]. Mtb, M. tuberculosis. ORF, Open reading frame. 


\subsection{Disinfectants for Bench Work}

Routine laboratory disinfectant techniques were practiced each time while dealing bacterial cultures and prior to assay set up with mycobacteria i.e.

- Use of bench-top $70 \%$ ethanol.

- Trigene solution (for mycobacteria).

- UV light for at least 15 minutes followed by disinfection of hood area.

- $5-10 \%$ bleach is used to inactivate/kill bacterial cultures after assay has been set up, before washing and autoclaving each time.

A routine laboratory practice of wiping surfaces and Biological Safety Cabinets with $70 \%$ ethanol may not be sufficient for inactivating M. tuberculosis, due to its thick cell wall, it can withstand weak disinfectants [46]. However, Trigene 50\% (v/v) or Hydrogen Peroxide Vapour (HPV) is effective to completely disinfect surfaces and hood facility from $M$. tuberculosis contamination [523]. Therefore, all safety precautions including a thorough disinfection and prior sterilization of materials were achieved before setting up assays with $M$. tuberculosis. All surfaces were wiped afterwards with $70 \%$ ethanol and a UV light was left on for about half an hour. All cultures plates, flasks and troughs were suspended in $10 \%$ bleach before they were washed in a dishwasher and autoclaved for reuse.

\subsubsection{Culture Purity for Mycobacteria}

Due to a prolonged time period for mycobacterial cultures, contamination of $M$. bovis BCG with $M$. smegmatis is common in laboratories. Purity of the slow-growing mycobacterial culture was initially confirmed by performing a Zn stain and visualizing under oil immersion (100 x) objective (Figure 1.3). In order to differentiate from M. smegmatis growth, M. bovis BCG (or M. tuberculosis) culture was aseptically plated out on Luria-Bertani (LB) agar plates 
without antibiotics and plates were incubated at $37{ }^{\circ} \mathrm{C}$ for up to for 72 hours. Unlike $M$. smegmatis, $M$. bovis BCG requires additional growth nutrients (OADC), it does not grow on simple LB plates. Therefore, no growth on M. bovis BCG plates after 2 days confirmed culture purity and indicated no contamination with M. smegmatis or E.coli. In contrast, both E.coli and M. smegmatis start to grow on LB agar plates between 24-48 hours. 


\subsection{Miscellaneous Figures}

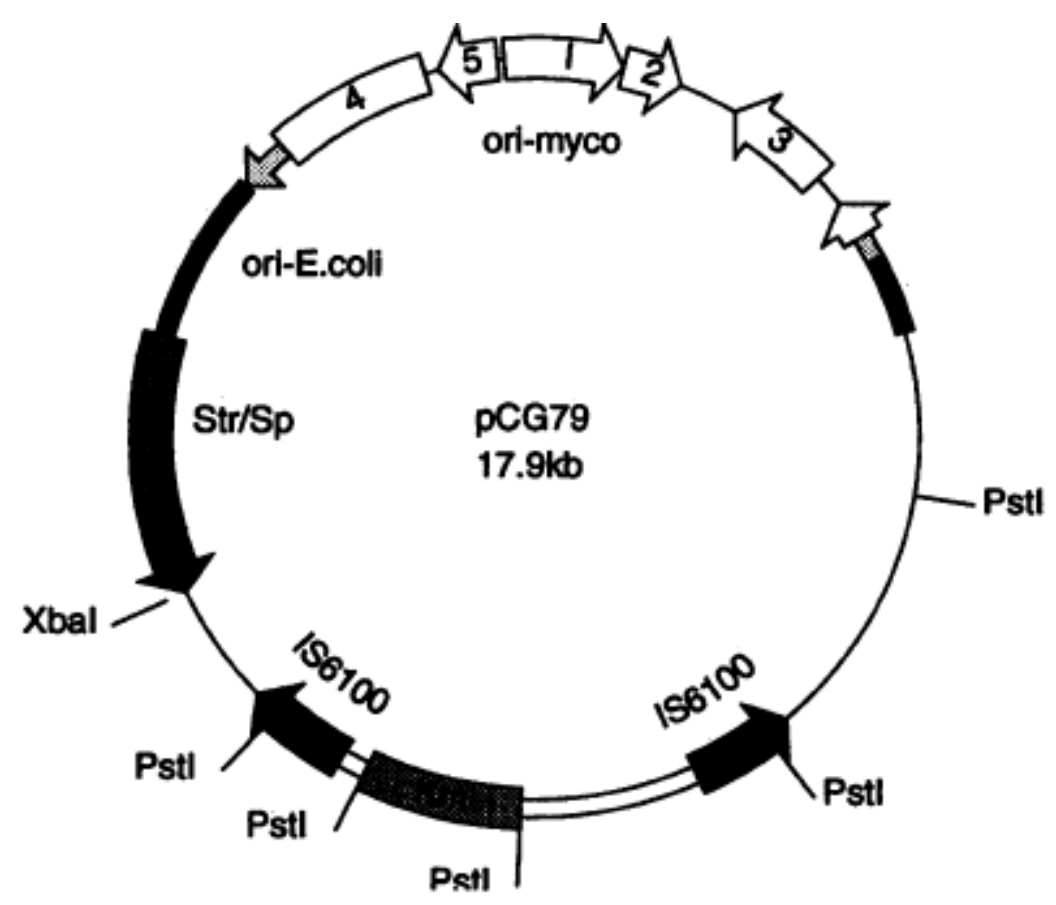

Figure 7.1: (A) Map for pCG79 [248]. 


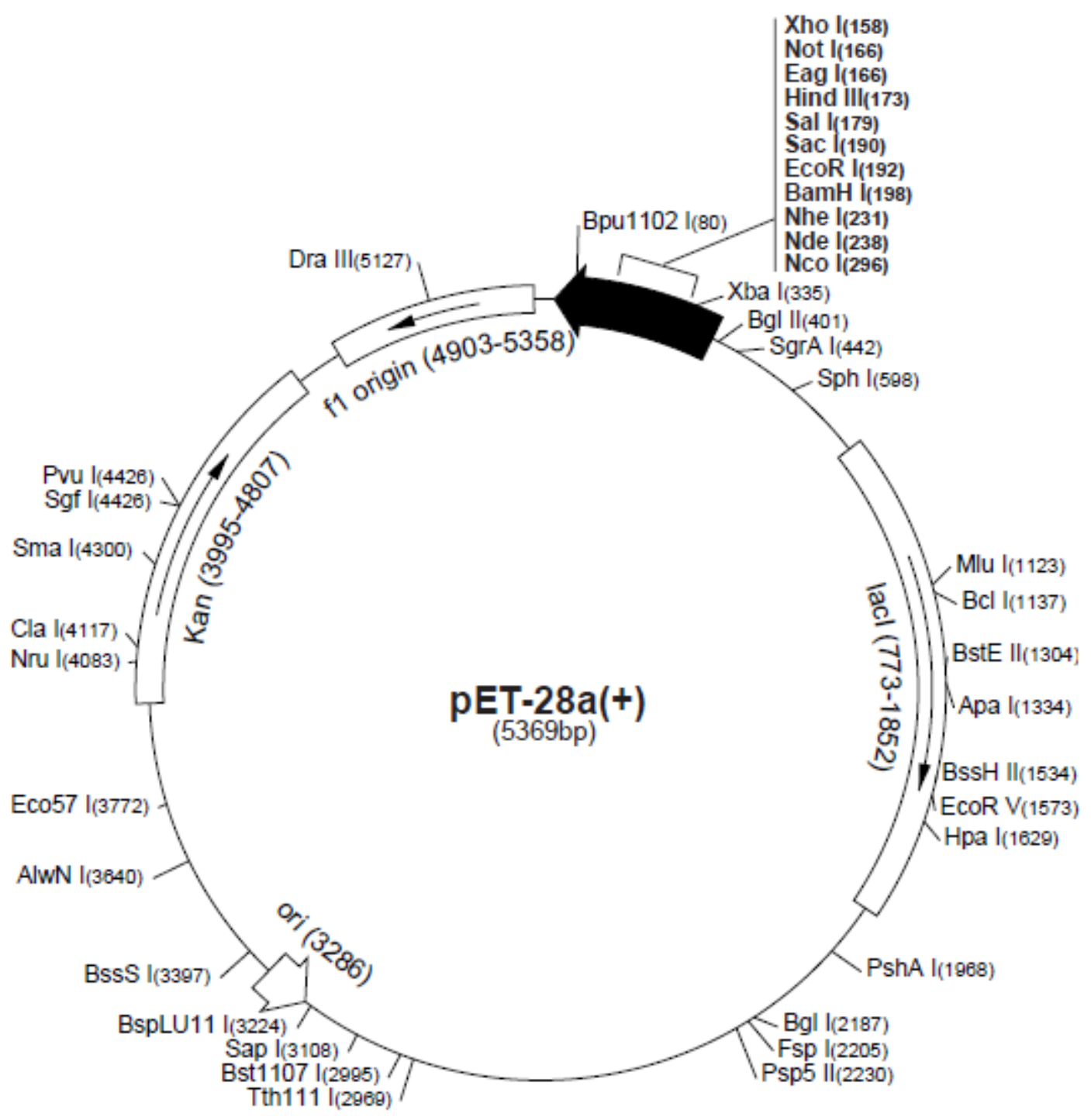

Figure 7.1: (B) Map for pET28a (+) [524]. 


\begin{tabular}{|c|c|c|}
\hline Query & 2 & 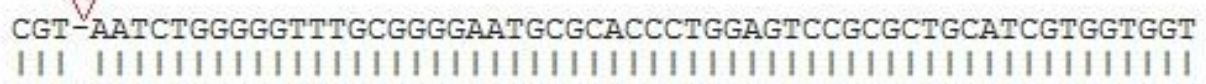 \\
\hline Sbjet & 34 & CGTAAATCTGGGGGTTTGCGGGGAATGCGCACCCTGGAGTCCGCGCTGCATCGTGGTGGT \\
\hline Query & 61 & 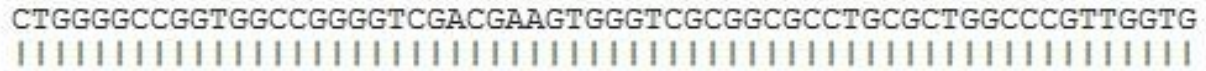 \\
\hline Sbjet & 94 & CTGGGGCCGGTGGCCGGGGTCGACGAAGTGGGTCGCGGCGCCTGCGCTGGCCCGTTGGTG \\
\hline Query & 121 & 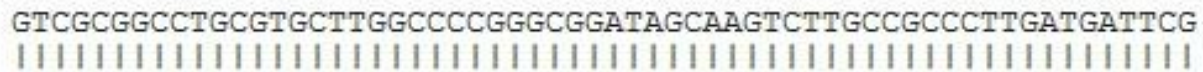 \\
\hline Sbjet & 154 & GTCGCGGCCTGCGTGCTTGGCCCCGGGCGGATAGCAAGTCTTGCCGCCCTTGATGATTCG \\
\hline Query & 181 & 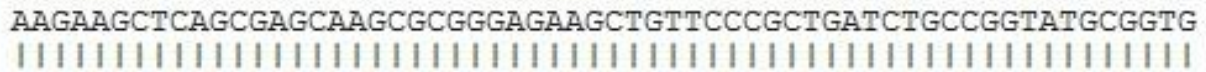 \\
\hline Sbjet & 214 & AAGAAGCTCAGCGAGCAAGCGCGGGAGAAGCTGTTCCCGCTGATCTGCCGGTATGCGGTG \\
\hline Query & 241 & 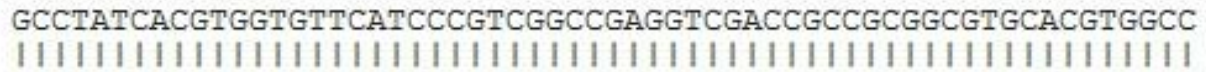 \\
\hline Sbjet & 274 & GCCTATCACGTGGTGTTCATCCCGTCGGCCGAGGTCGACCGCCGCGGCGTGCACGTGGCC \\
\hline Query & 301 & 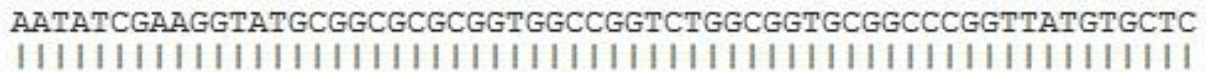 \\
\hline Sbjet & 334 & AATATCGAAGGTATGCGGCGCGCGGTGGCCGGTCTGGCGGTGCGGCCCGGTTATGTGCTC \\
\hline Query & 361 & 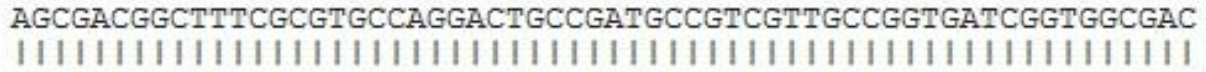 \\
\hline Sbjet & 394 & AGCGACGGCTTTCGCGTGCCAGGACTGCCGATGCCGTCGTTGCCGGTGATCGGTGGCGAC \\
\hline Query & 421 & $\begin{array}{l}\text { CAGCCGCCTGCATCGCCGCGGCTAGCGTGCTCGCCAAGGTCAGCCGGGACCGCGTG } \\
1111111111111111111111111111111111111111111111111\end{array}$ \\
\hline Sbjet & 454 & CAGCCGCCTGCATCGCCGCGGCTAGCGTGCTCGCCAAGGTCAGCCGGGACCGCGTG \\
\hline Query & 481 & $\begin{array}{l}\text { TTGCGTTGGACGCCGATCACCCCGGGTACGGTTTCGCGGAGCACAAGGGTTACAGC } \\
111111111111111111111111111111111111111111111111111\end{array}$ \\
\hline Sbjet & 514 & TTGCGTTGGACGCCGATCACCCCGGGTACGGTTTCGCGGAGCACAAGGGTTACAGC \\
\hline Query & 541 & $\begin{array}{l}\text { EGCACAGCCGGGCGCTGGCCCGATTGGGACCCTGCCCGCAGCACCGTTACTCG } \\
\|1\| 1\|1\| 1\|1\| 1\|1\| 1\|1\| 1\|1\| 1\|1\| 1\|1\| 1\|1\| 1\|1\| 1 \| 1\end{array}$ \\
\hline Sbjet & 574 & ECGGCGCACAGCCGGGCGCTGGCCCGATTGGGACCCTGCCCGCAGCACCGTTACTCG \\
\hline Query & 601 & 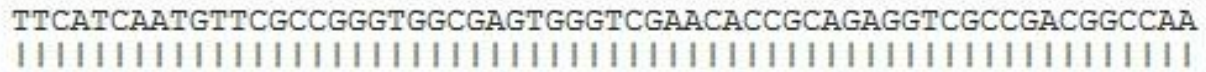 \\
\hline Sbjet & 634 & TTCATCAATGTTCGCCGGGTGGCGAGTGGGTCGAACACCGCAGAGGTCGCCGACGGCCAA \\
\hline Query & 661 & 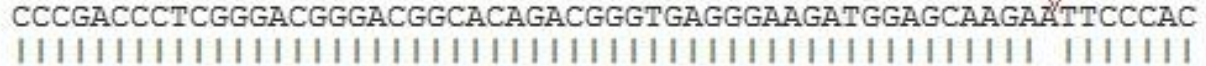 \\
\hline Sbjet & 694 & $\begin{array}{l}\text { CCCGACCCTCGGGACGGGACGGCACAGACGGGTGAGGGAAGATGGAGCAAGAGTTCCCAC } \\
\Omega\end{array}$ \\
\hline Que: & 721 & 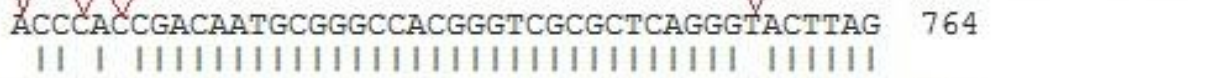 \\
\hline & 75 & GCGCTCAGGGCACTTAG \\
\hline
\end{tabular}

Figure 7.2: Sequencing results of cloned Rv2228c into pET28a (+). Red arrows show mis-matches, gaps and substitutions within in the insert (Rv2228c) due to low fidelity of the polymerase enzyme used in the PCRamplification of Rv2228c. Analysis was not taken further to the protein purification stage as the mutations might have truncated the desired protein. However, a biochemical assay at the University of Auckland was used instead to confirm the target for selected RNase HI inhibitors and results have been added in section 5.4.3. See 
Figures 5.10 and 7.14 for more details regarding in silico interaction of inhibitors with RNase HI and confirmation of this protein as a target for these inhibitors in M. tuberculosis, respectively.

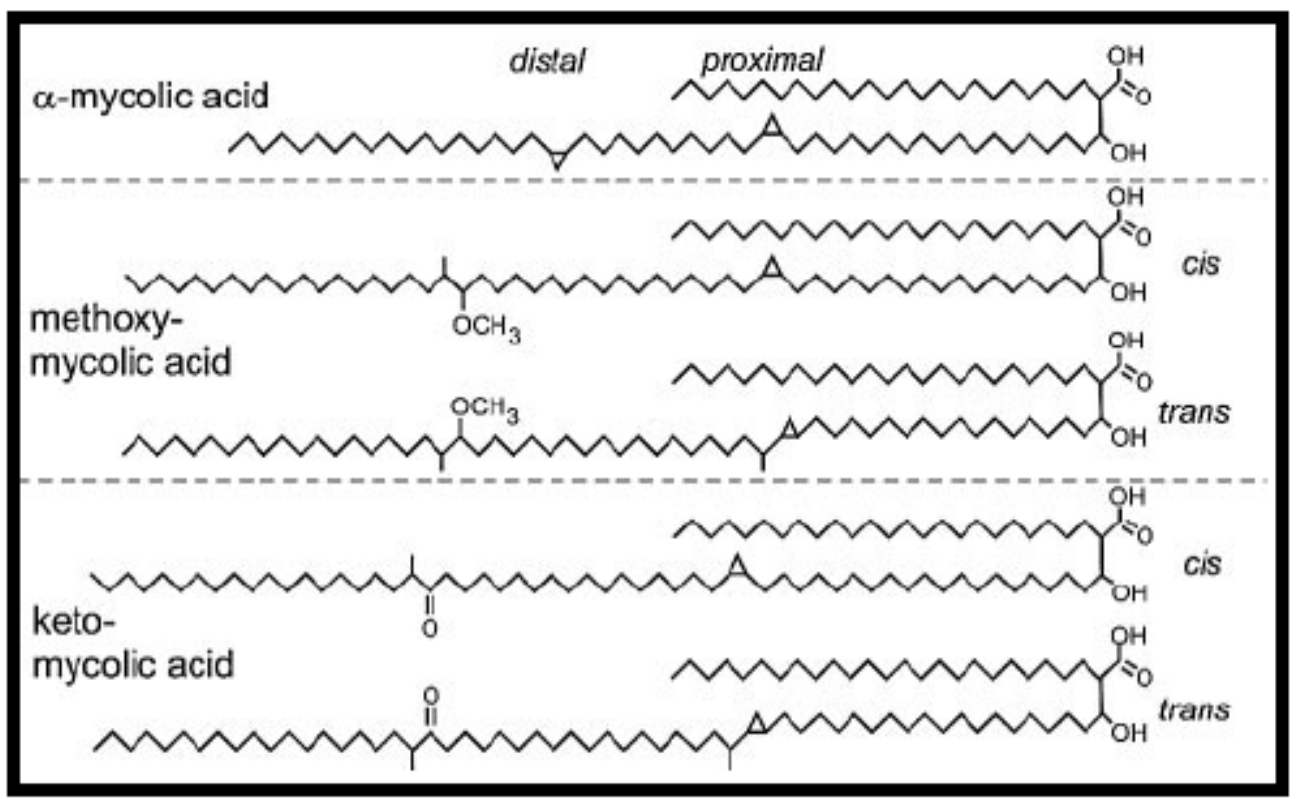

Figure 7.3: (I) Types of mycolic acids in M. tuberculosis showing cis and trans configuration. Figure adapted with permission from Takayama. et al. [525].

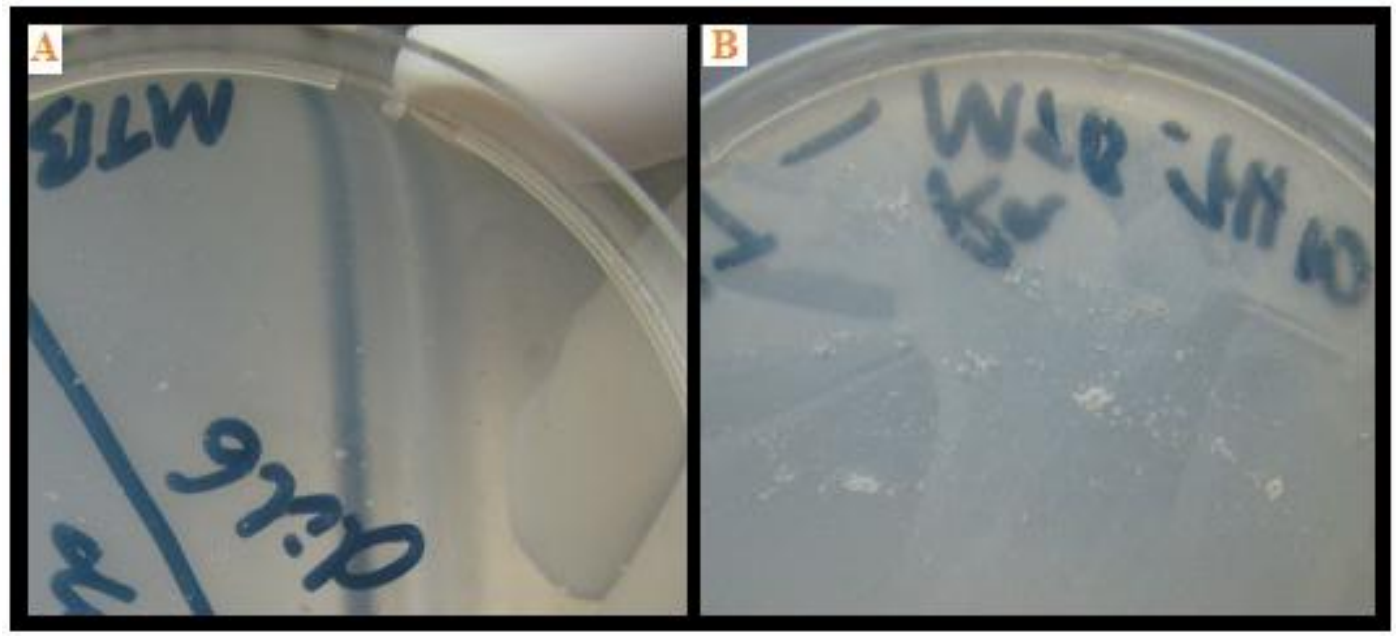

Figure 7.3: (II) M. tuberculosis H37Ra growth on solid media, 7 H10 plates supplemented with $10 \%$ OADC (A) Negative control - no growth and (B) mycobacterial growth after three weeks of incubation. 


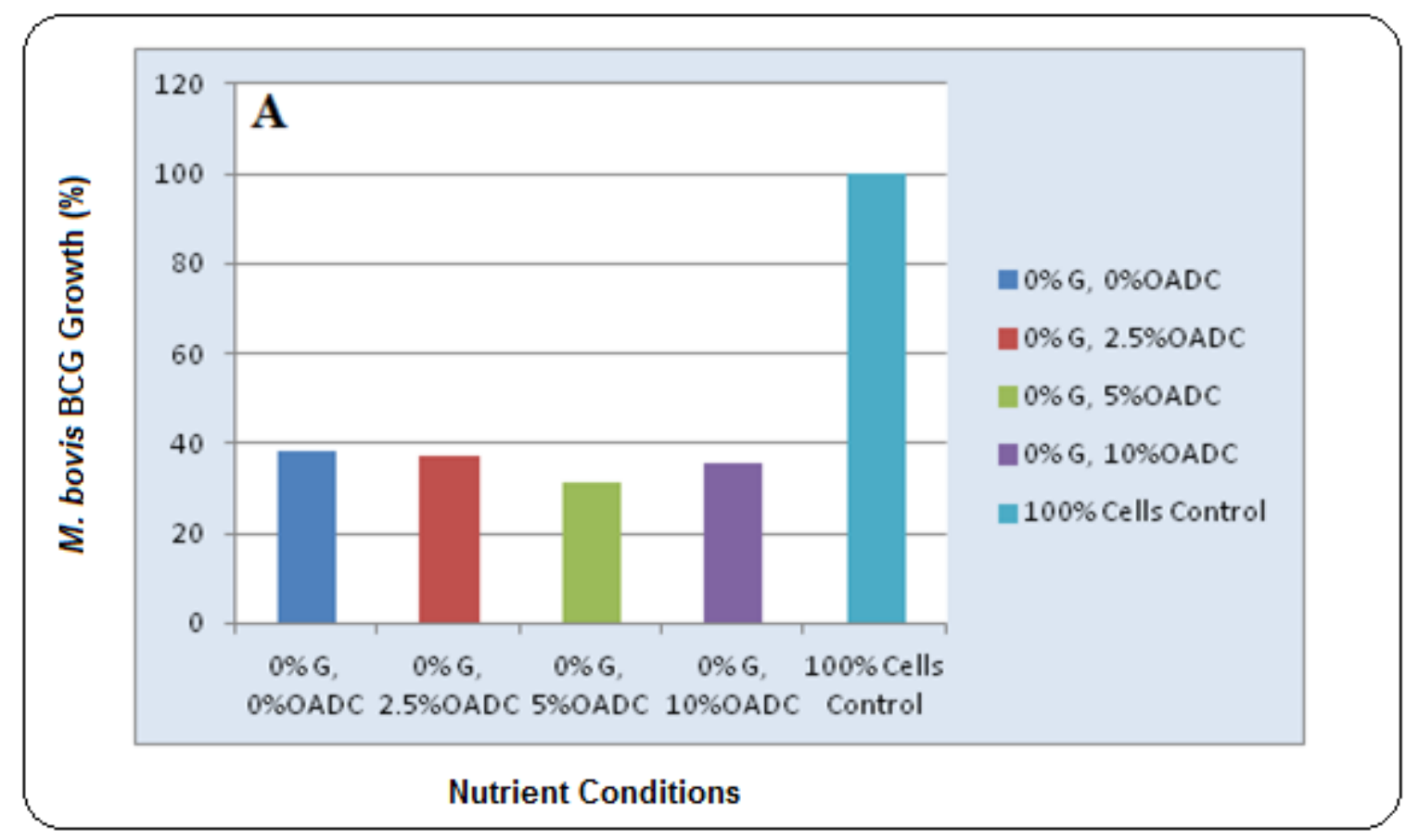

Figure 7.4: (A) Carbon starvation in M. bovis BCG. Different concentrations of OADC were used with no glycerol. BCG growth was significantly reduced by keeping 10\% OADC but removing glycerol, compared to standard nutrient conditions ( $100 \%$ cells control).

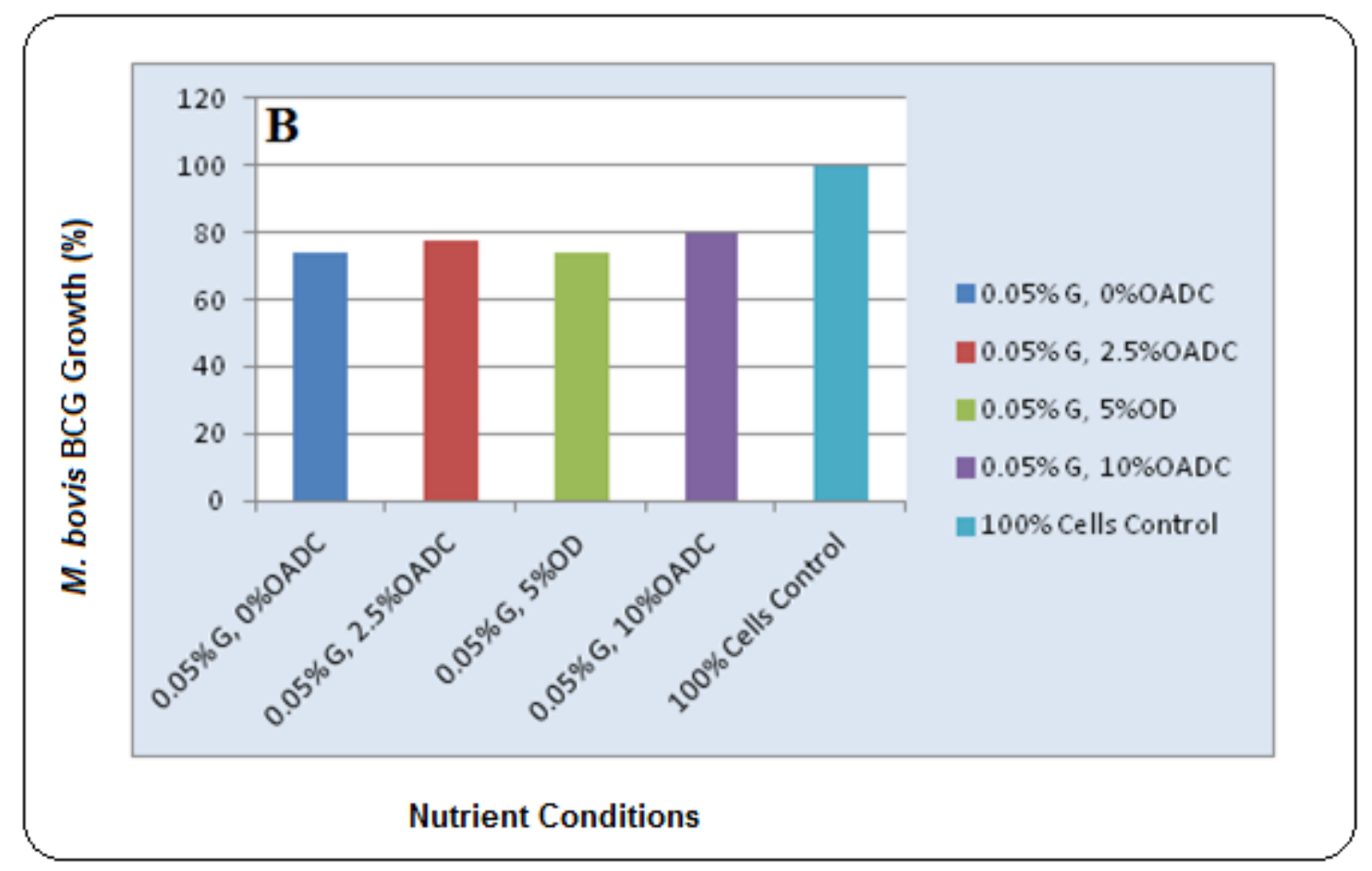

Figure 7.4: (B) Carbon starvation in M. bovis BCG. Different concentrations of OADC were used along with $0.05 \%$ glycerol. 


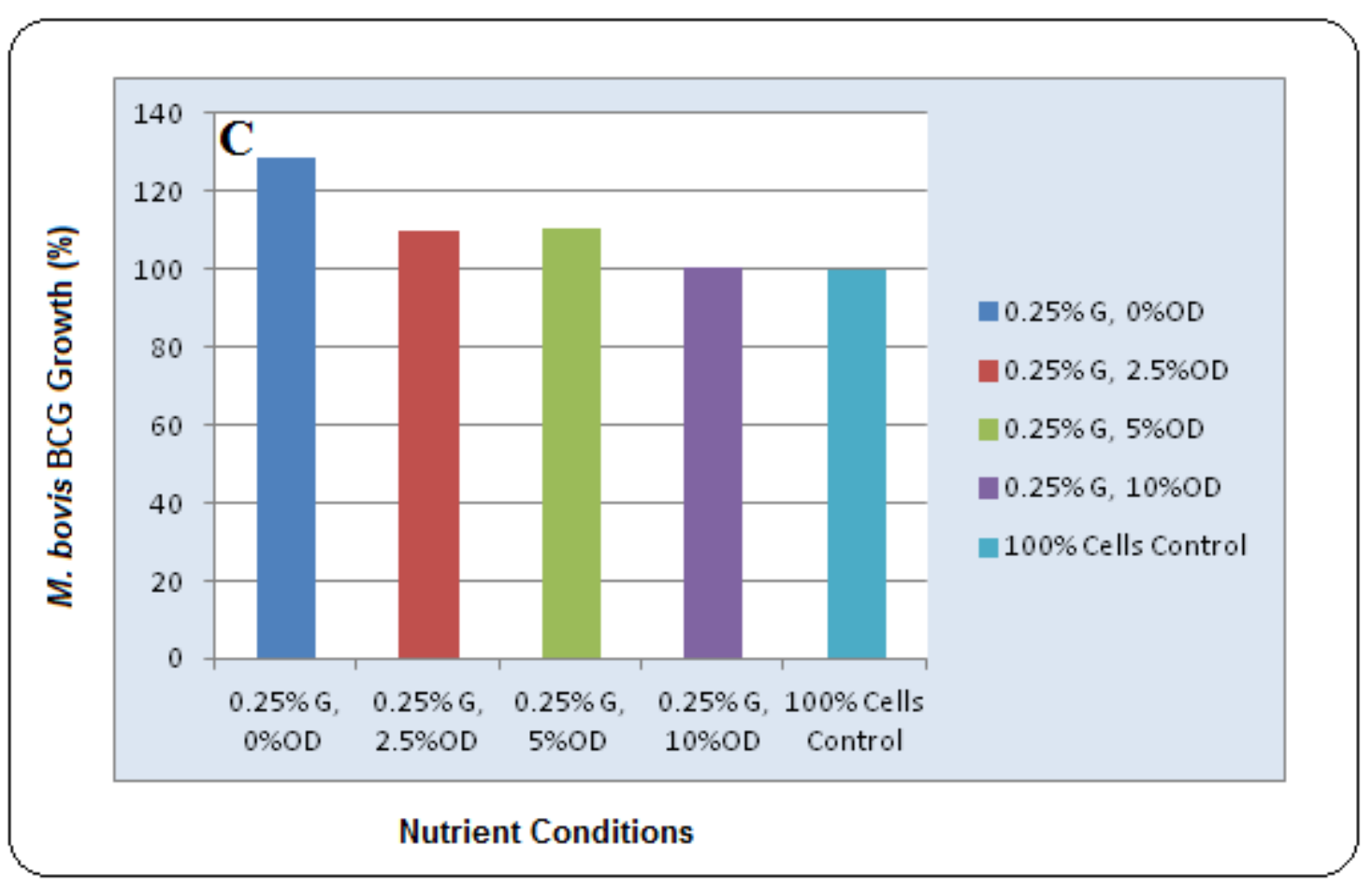

Figure 7.4: (C) Carbon starvation in M. bovis BCG. Different concentrations of OADC were used along with $0.25 \%$ glycerol. Glycerol appeared to be the nutrient-limiting factor for BCG growth. OD represents OADC in the figure (see section 2.2.1.1 for more detail on mycobacterial growth supplements).

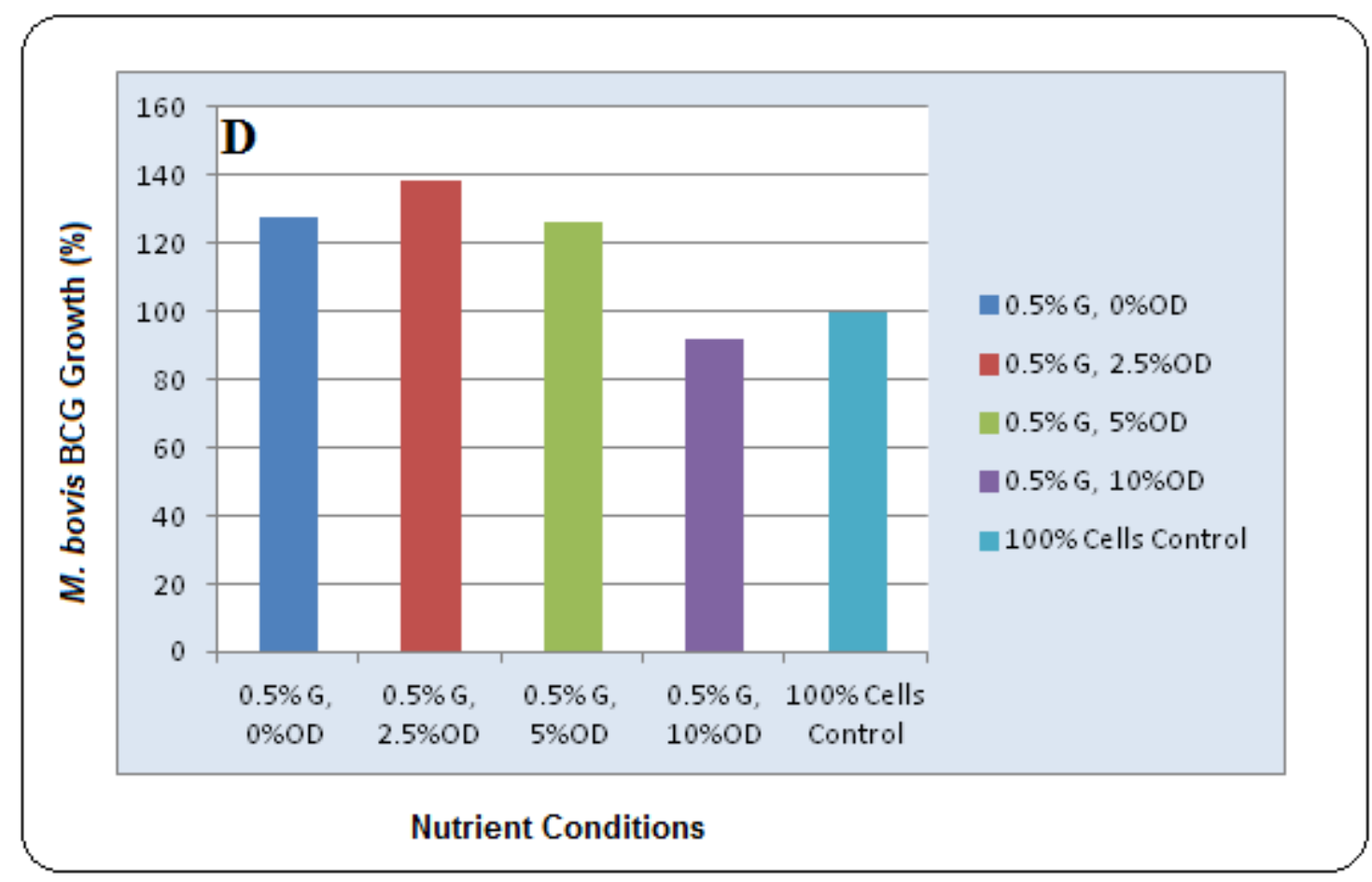

Figure 7.4: (D) Carbon starvation in M. bovis BCG. Different concentrations of OADC were used along with $0.5 \%$ glycerol. OD represents OADC in the figure. 
Figure 7.5 (A-F): Dose-response curves for standard anti-Tb drugs against $M$. bovis BCG.
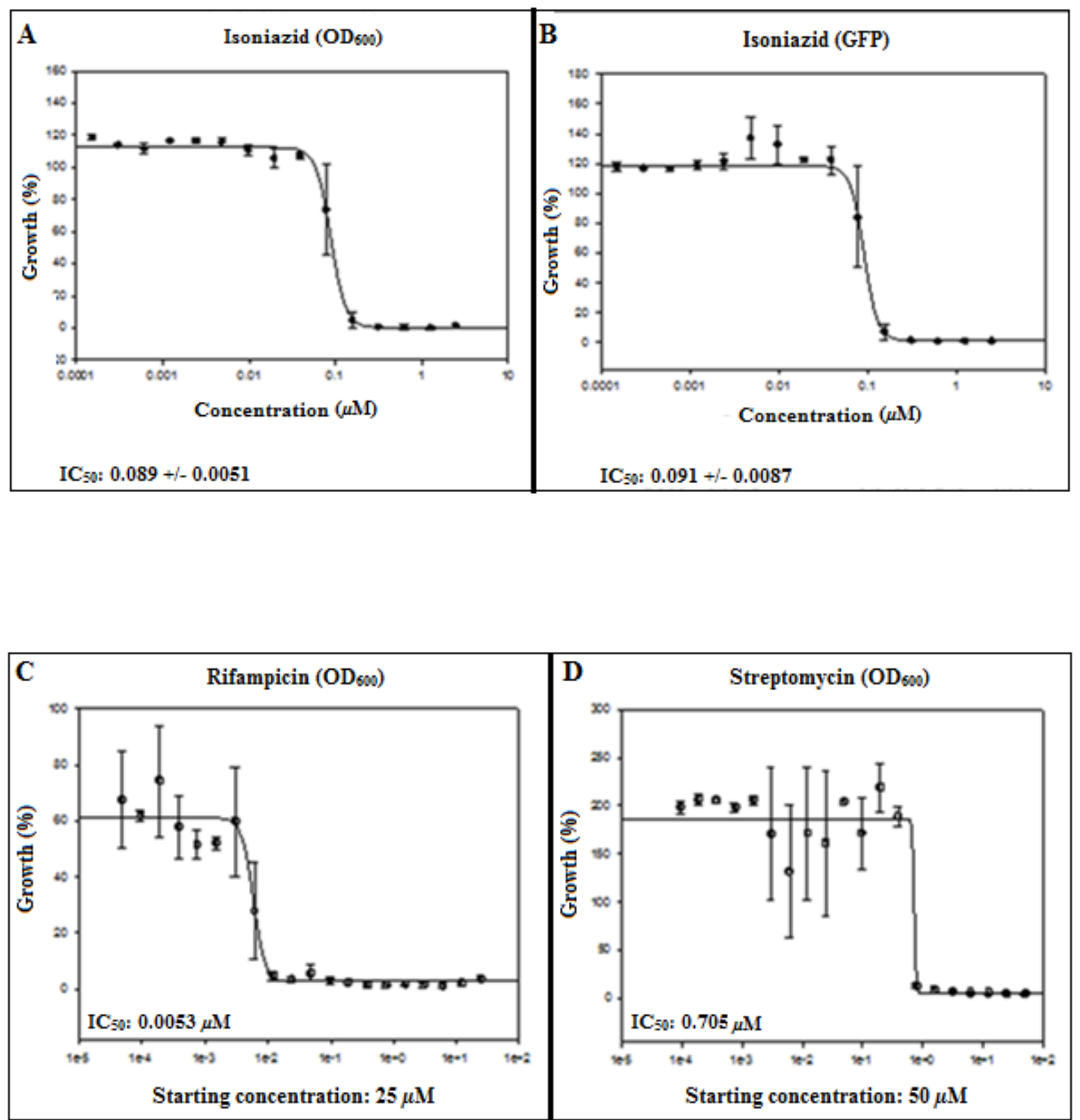

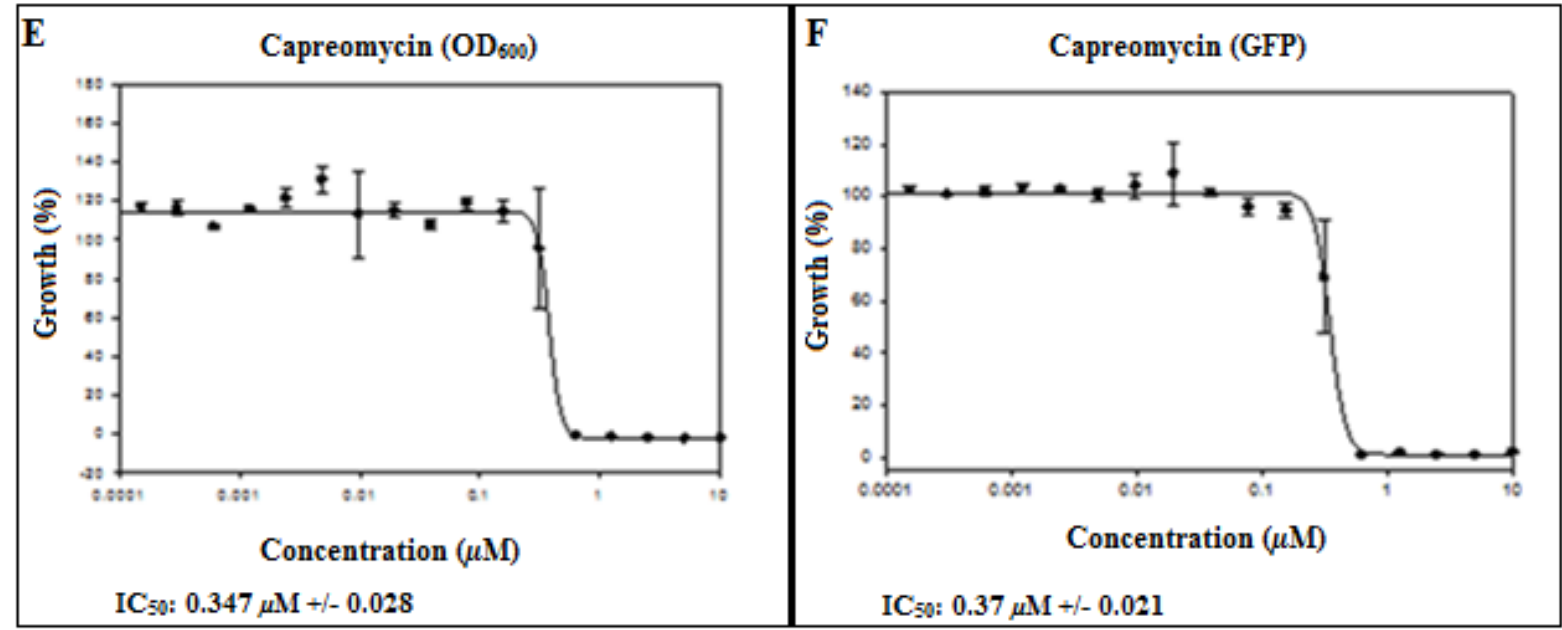

Figure 7.5 (A-F): Dose-response curves for standard anti-Tb drugs against $M$. bovis BCG.

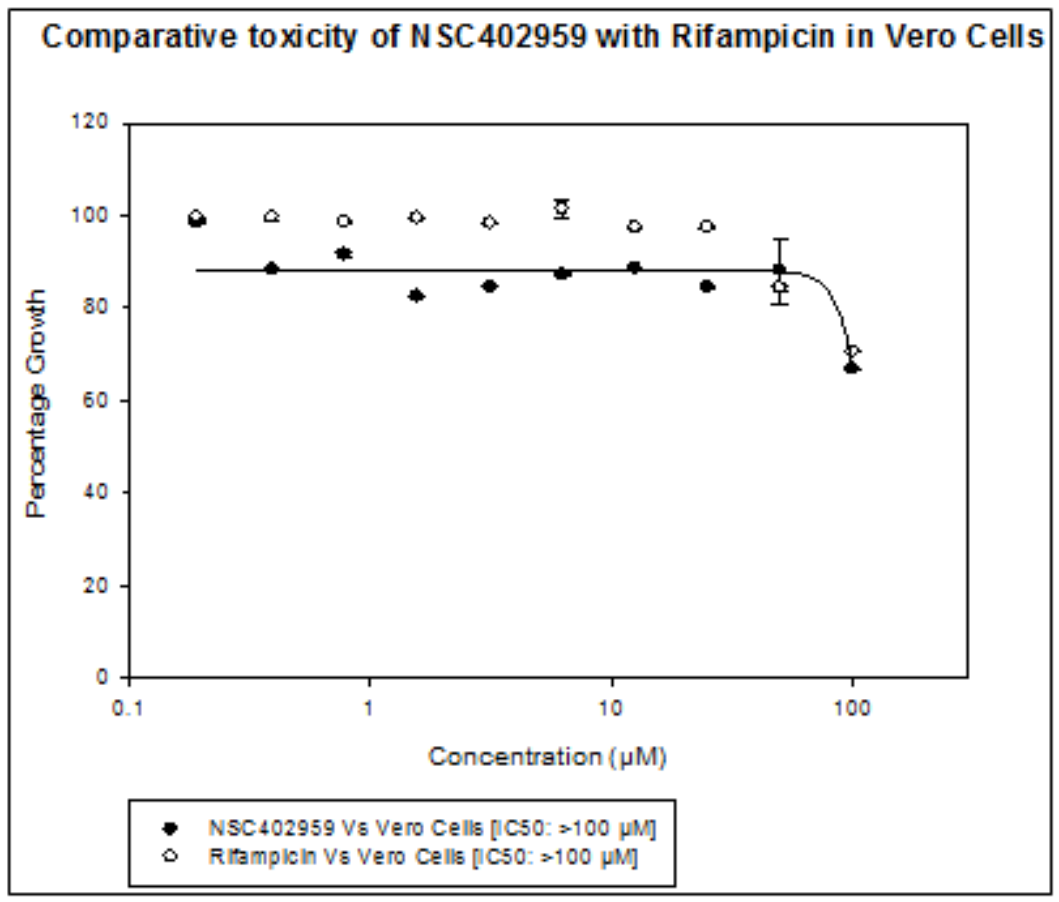

Figure 7.6: Cytotoxicity of NSC402959 toward the Vero cell line compared to the front-line anti-Tb drug, rifampicin. Both compounds exhibited toxicities in an acceptable range $\left(\mathrm{IC}_{50}>100 \mu \mathrm{M}\right)$. 


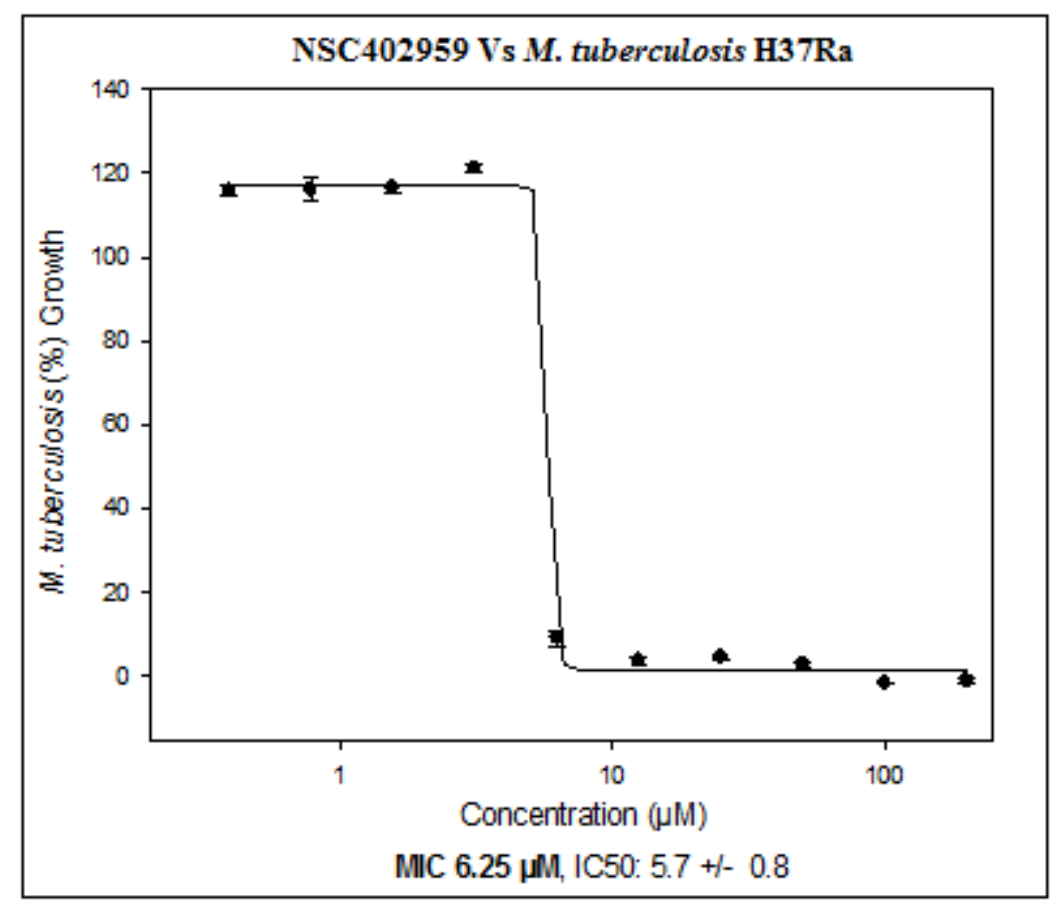

Figure 7.7: Anti-mycobacterial activity of NSC402959 against M. tuberculosis H37Ra under standard nutrient-rich conditions. Anti-mycobacterial activity of NSC402959 was found to be within the range of 6.25 $\mu \mathrm{M}$ to $12.5 \mu \mathrm{M}$ in dose-response assays with nutrient-rich conditions.

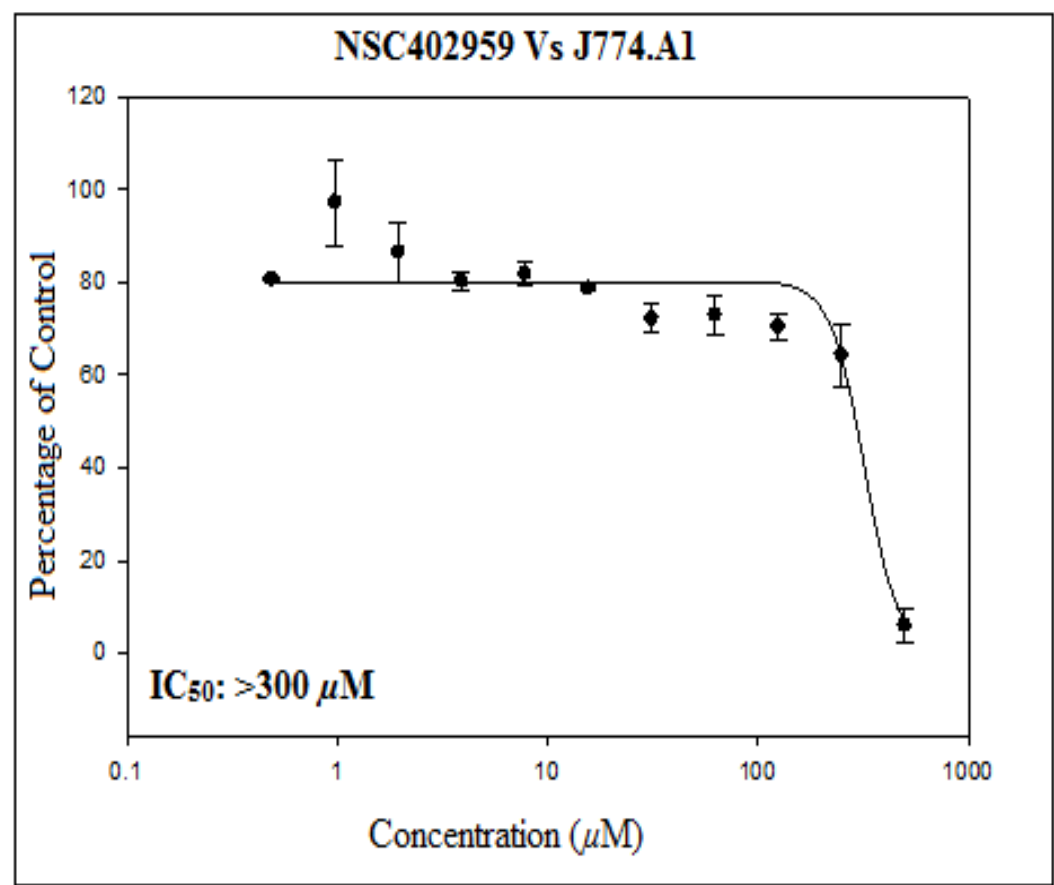

Figure 7.8: Cytotoxicity of NSC402959 against the J774.A1 cell line. 


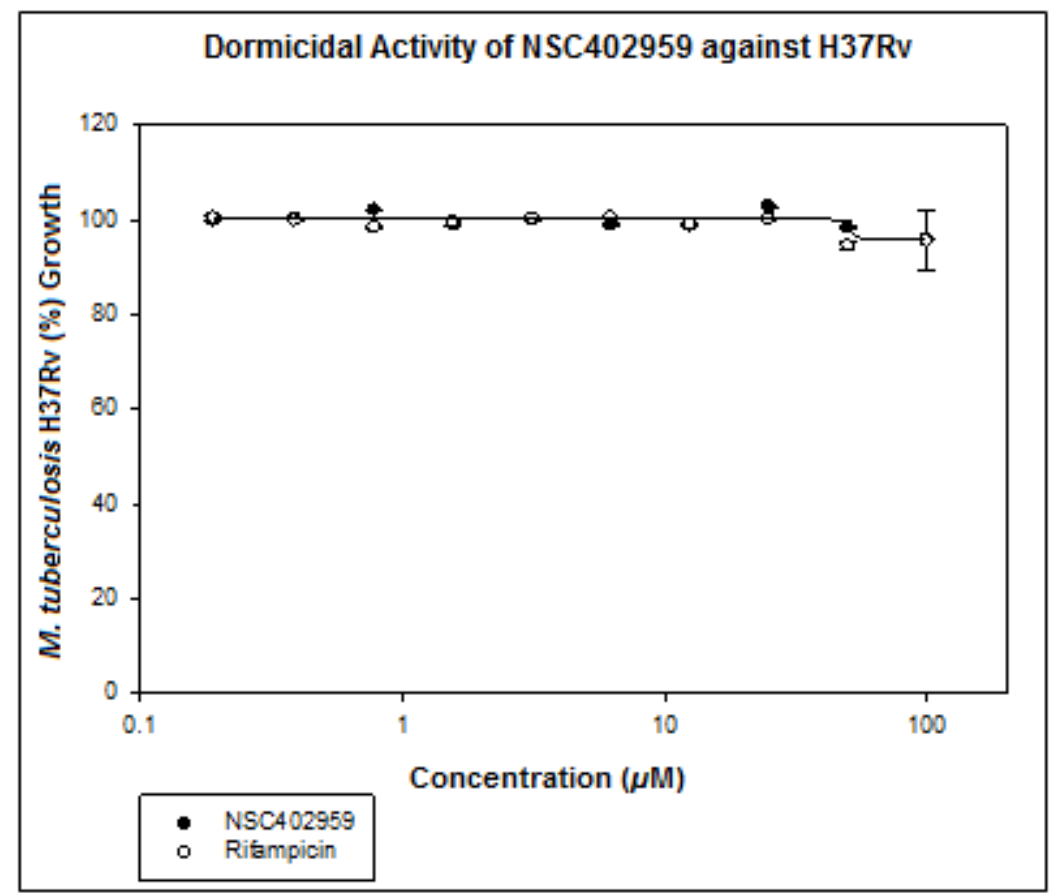

Figure 7.9: Dormicidal activity of $M$. tuberculosis inhibitor, NSC402959 and a standard Tb drug, rifampicin. No dormicidal activity for NSC402959 was observed against H37Rv cells at concentrations up to $100 \mu \mathrm{M}$. The results for NSC402959 were comparable to rifampicin which was tested in parallel and it inhibited growth of all mycobacterial strains at $12.5 \mu \mathrm{M}$ in the growth inhibition assays. 


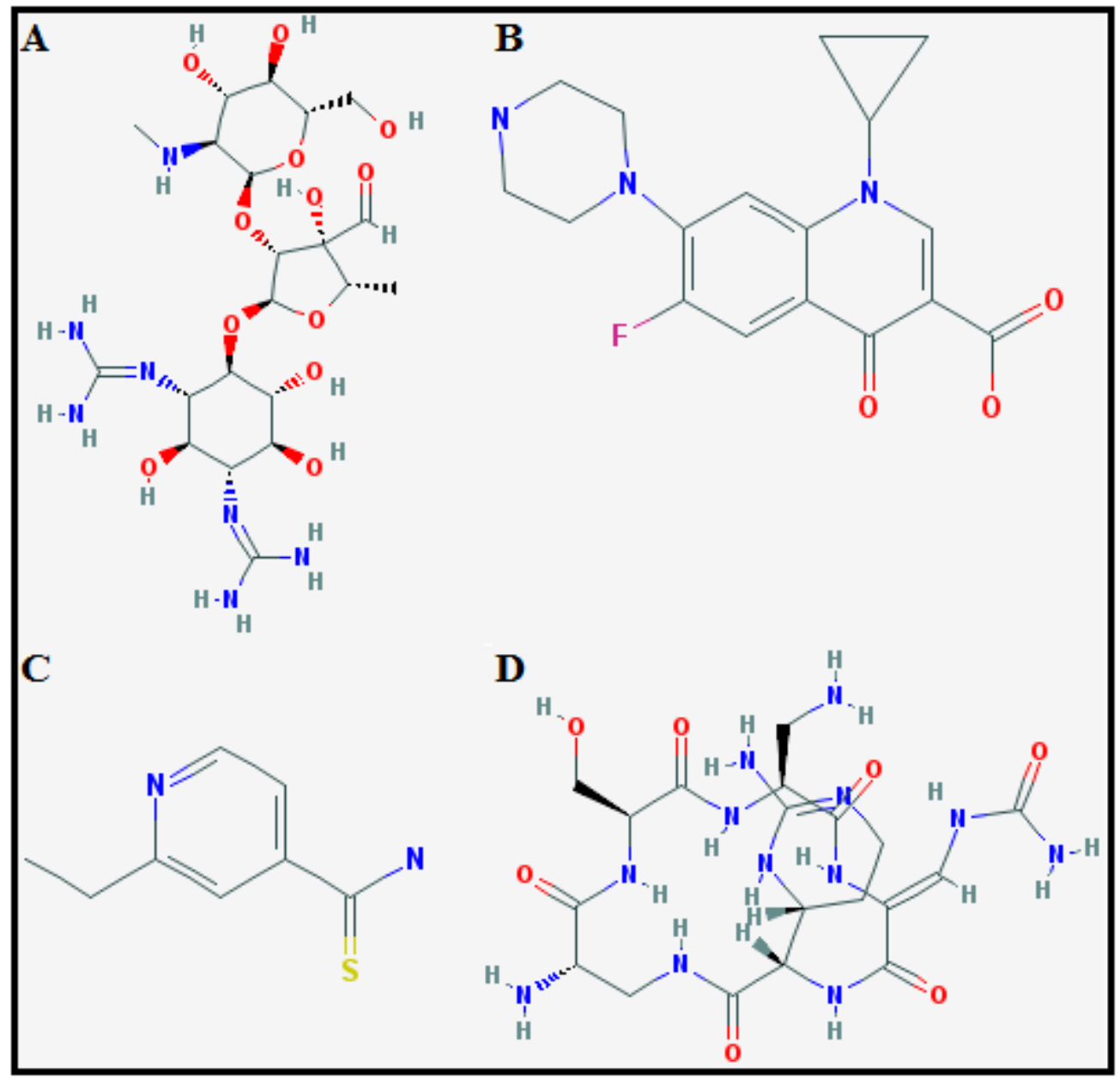

Figure 7.10: Standard second-line anti-Tb drugs (A) Streptomycin, $M F C_{21} \mathrm{H}_{39} \mathrm{~N}_{7} \mathrm{O}_{12}$ and $M W 581.57$ g/mol (B) Ciprofloxacin, $\mathrm{MF} \mathrm{C}_{17} \mathrm{H}_{18} \mathrm{FN}_{3} \mathrm{O}_{3}$ and MW $385.8 \mathrm{~g} / \mathrm{mol}$ (C) Ethionamide, $\mathrm{MF}_{8} \mathrm{H}_{10} \mathrm{~N}_{2} \mathrm{~S}$ and MW $166.24 \mathrm{~g} / \mathrm{mol}$ and (D) Capreomycin IIA, MF $\mathrm{C}_{19} \mathrm{H}_{32} \mathrm{~N}_{12} \mathrm{O}_{7}$ and MW $540.53 \mathrm{~g} / \mathrm{mol}$ (PubChem database). 


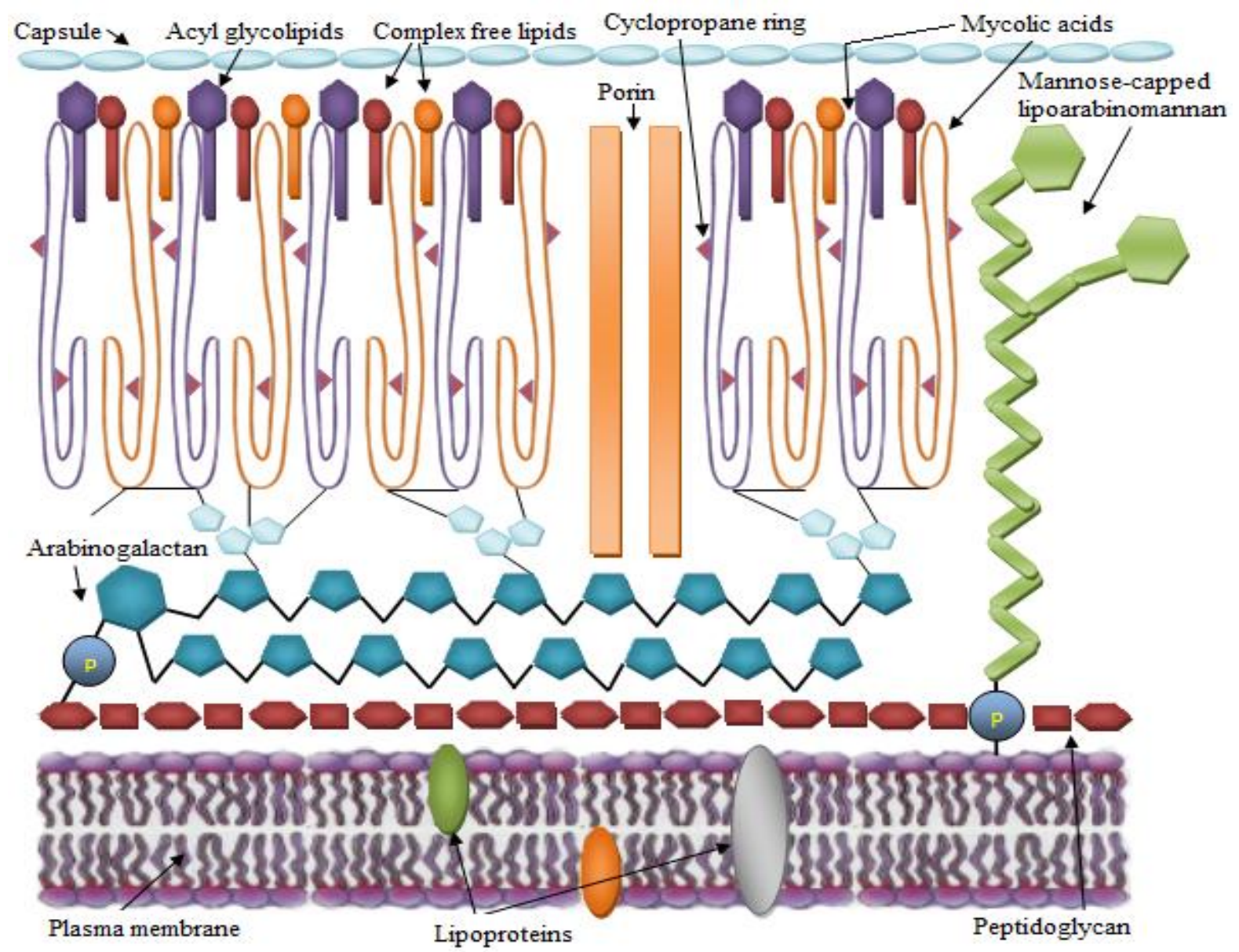

Figure 7.11: M. tuberculosis cell wall revealing a complex structure and arrangement of glycolipids and capsule. Figure reproduced with permission from Riley LW, 2006 [526]. 


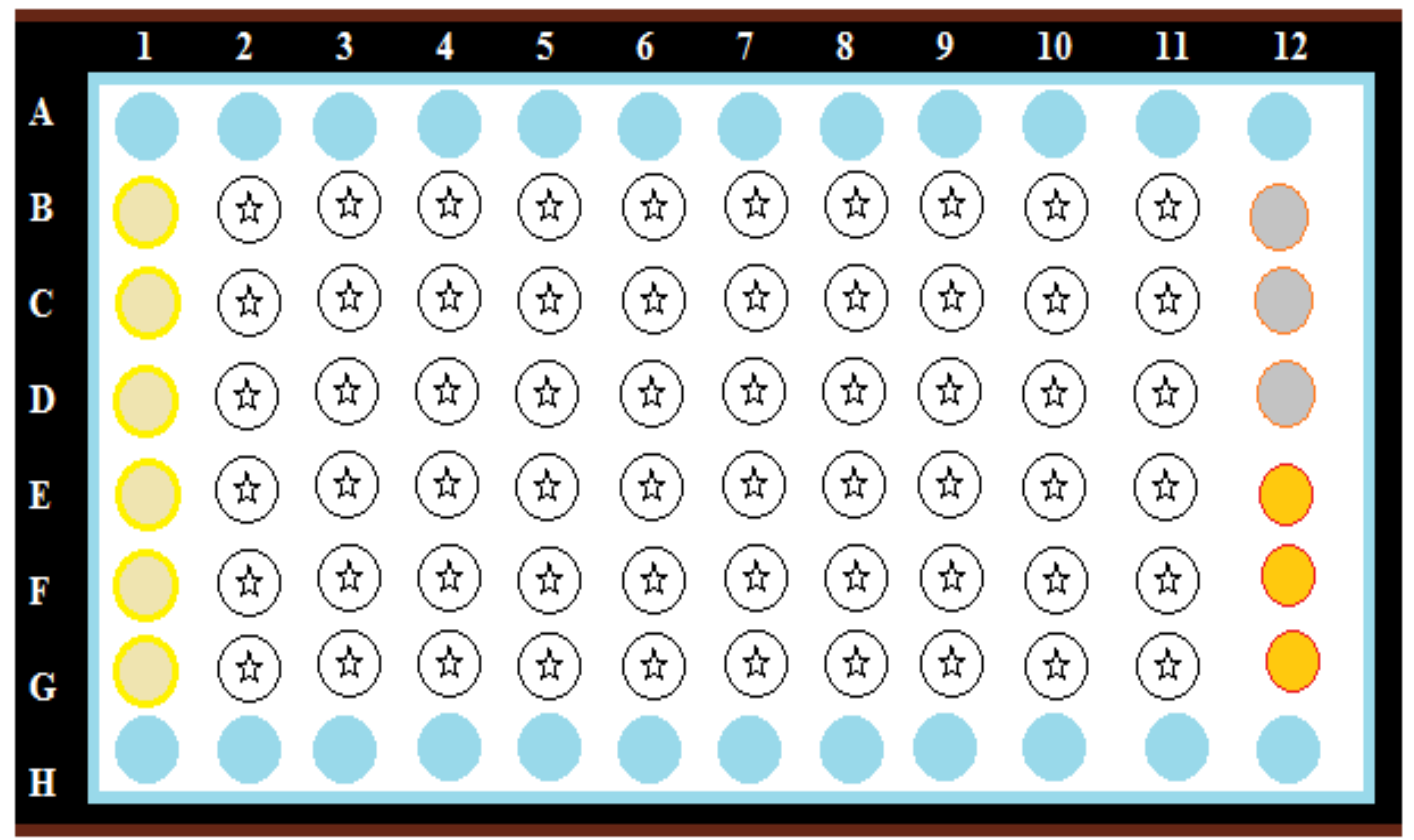

Legend: $\rightarrow$ media + cells, $\rightarrow$ positive control (RIF), $\rightarrow$ media only (4) $\rightarrow$ compound + cells $\quad \rightarrow$ sterile water

Figure 7.12: Plate map for validation of growth inhibitors in bacteriostatic assays. The arrangement of positive (anti-Tb drug + cells) and negative controls (medium only) used in bacteriostatic and dormicidal assays was similar. 


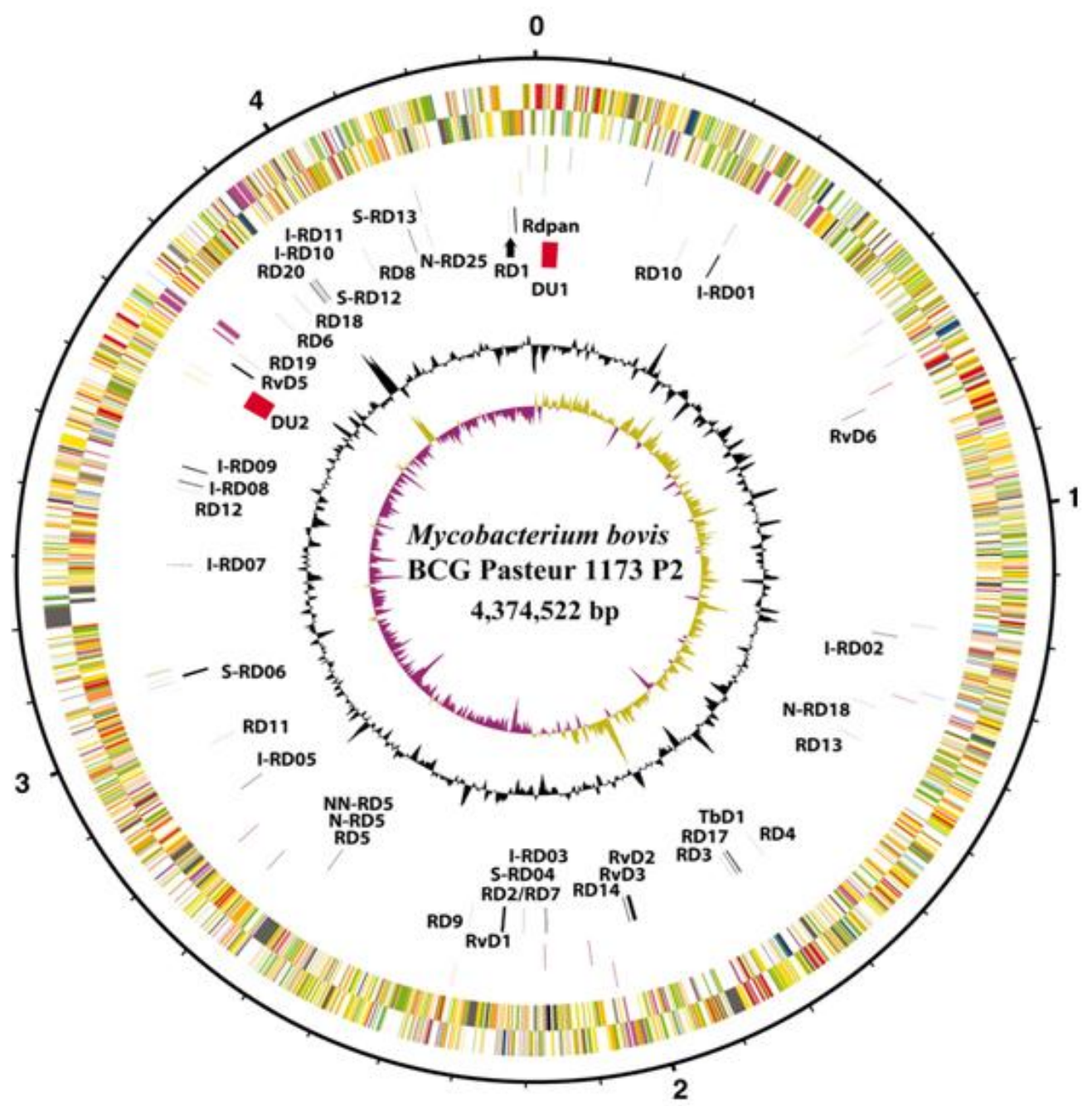

Figure 7.13: Genomic map of $M$. bovis BCG Pasteur 1173P2. (Figure was adapted with permission from [357]). 


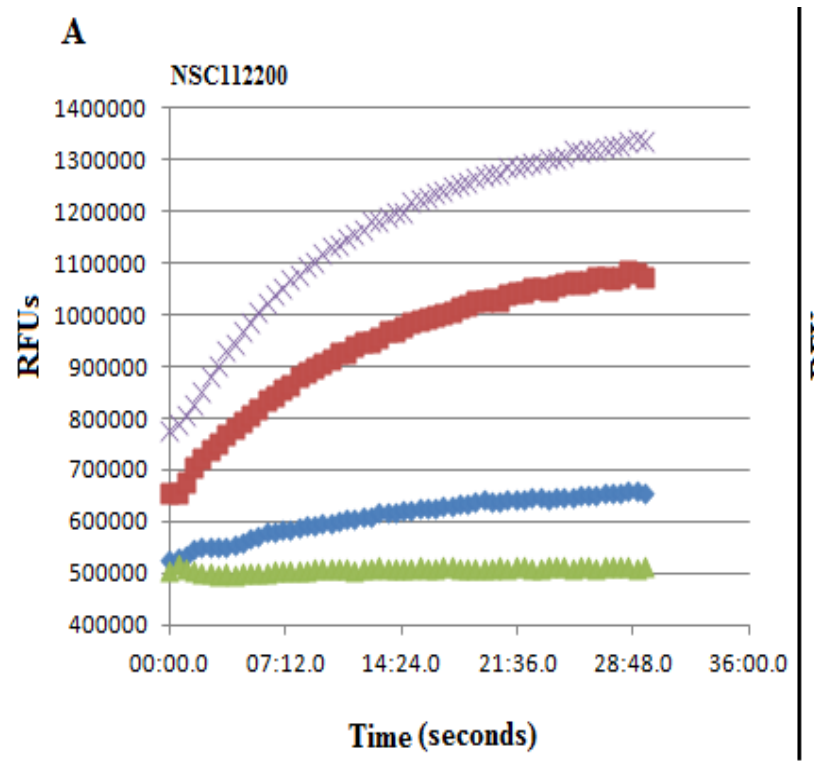

$\checkmark 100 \mu \mathrm{M} \quad \square$ - $5 \mu \mathrm{M} \quad \Delta$ blank $\times 100 \%$ control

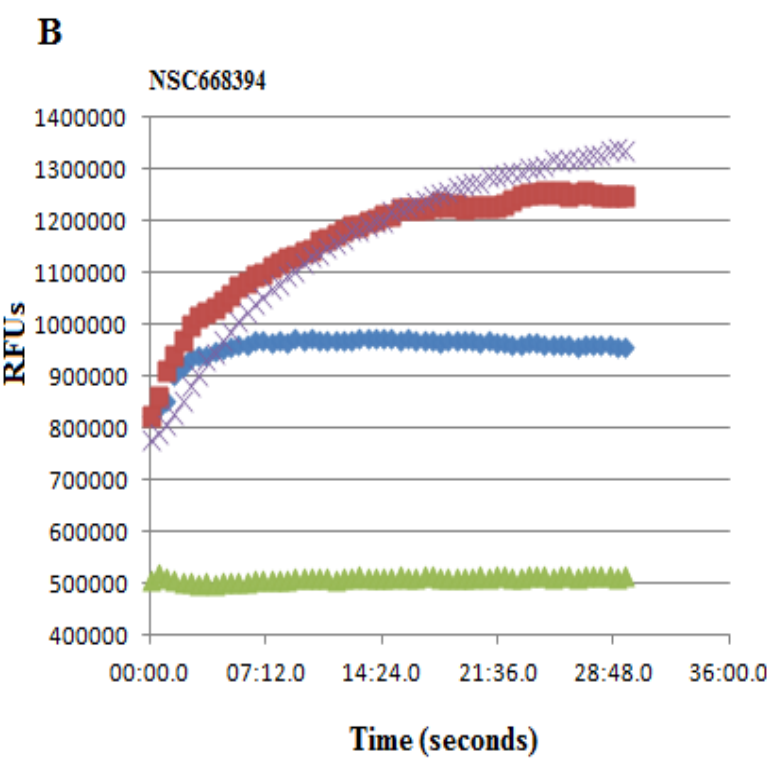

$\downarrow 100 \mu \mathrm{M} \quad \square 5 \mu \mathrm{M} \quad \Delta$ blank $\times 100 \%$ control

Figure 7.14: Activity of RNase H inhibitors (A) NSC112200 and (B) NSC668394, against purified RNase HI (Rv2228c) from M. tuberculosis. Both inhibitors were tested at two test concentrations (100 $\mu \mathrm{M}$ and $5 \mu \mathrm{M})$ with controls. A preliminary analysis of the activity of these inhibitors indicated that NSC112200 (MIC 6.25 $\mu \mathrm{M}$ against M. tuberculosis in dose response assay) specifically inhibits the RNase HI (Rv2228c) from M. tuberculosis $\mathrm{H} 37 \mathrm{Rv}$ compared to NSC668394 (MIC $3.125 \mu \mathrm{M}$ against M. tuberculosis in dose response assay). NSC112200 does not interfere with human RNase H (PubChem database). The 100\% positive control (same WT enzyme at $4 \mathrm{nM}$ ) and blank (DNA/RNA hybrid at $100 \mathrm{nM}$ ) curves have been superimposed on each set of inhibitor data. Assay was carried out at $21^{\circ} \mathrm{C}$ for 36 minutes with readings every 30 seconds. It is an initial assessment of these inhibitors for the identification of RNase HI as their primary target in M. tuberculosis and results are based on one experiment only (Figure courtesy of Dr. Stephanie Dawes), also see figure 5.10 for compound NSC112200. 